\title{
CRUST AND UPPER MANTLE INHOMOGENEITIES BENEATH WESTERN \\ NORTH ISLAND, NEW ZEALAND: EVIDENCE FROM SEISMOLOGICAL AND ELECTROMAGNETIC DATA
}

\author{
By \\ Michelle Linda Salmon
}

A thesis

submitted to the Victoria University of Wellington in fulfilment of the requirements for the degree of

Doctor of Philosophy in Geophysics. 



\section{ABSTRACT}

Three geophysical techniques have been used to investigate the location and the nature of a large-scale change in crust and uppermost mantle properties below the western North Island of New Zealand. Receiver function analysis reveals a step like change in crustal thickness from $\sim 25 \mathrm{~km}$ below the northwestern North Island to $\geqslant 32 \mathrm{~km}$ in the southwestern North Island. P-wave attenuation is elevated north of this change in crustal thickness $\left(1000 / Q_{p} \approx 1.9\right.$ for $\left.\alpha=0\right)$ and is compatible with a wet mantle at near solidus temperatures ( $\mathrm{T} \approx 0.97$ melting temperature). Attenuation decreases by at least a factor of 2 for the southwestern North Island to values closer to those expected for normal continental lithosphere $\left(1000 / Q_{p} \leqslant 1\right.$ for $\left.\alpha=0\right)$. A region of extremely high attenuation $\left(1000 / Q_{p} \approx 5\right.$ for $\left.\alpha=0\right)$ is observed below the Central Volcanic Region. This value of attenuation is compatible with a wet mantle at temperatures just above melting ( $\mathrm{T} \approx 1.02$ melting temperature). Finally $2 \mathrm{D}$ modelling of magnetotelluric data reveals a region of low electrical resistivity $(100 \Omega \mathrm{m})$ in the mantle below the region of thinned crust. Like the $\mathrm{P}$-wave attenuation, this region of low resistivity can be explained by a water-saturated mantle at near solidus temperatures $(\mathrm{T}=0.88-0.97$ melting temperature).

The changes in crustal thickness, attenuation and electrical resistivity are all coincident with the southern limit of volcanism $\left(\sim 39.3^{\circ} \mathrm{S}\right)$ at a boundary that runs approximately east-west, perpendicular to the present plate boundary. The only surface expressions of this boundary are the termination of volcanism and the dome-like uplift of the North Island, which has previously been explained by the presence of a buoyant lowdensity mantle beneath the northwestern North Island. Elevated temperatures and water content inferred from this study are in agreement with this explanation.

The sudden transition displayed in all three data sets, but particularly the crustal thickness step seen in the receiver function, calls for a special explanation. Thermal processes are too diffuse to explain the step and instead a mechanical process is called for. One possibility is that the step was created by convective removal of thickened lithosphere. 


\section{ACKNOWLEDGEMENTS}

At Victoria University of Wellington I would like to thank Martha Savage and Tim Stern for their support and supervision during this project. Thank you also to Malcolm Ingham for all the help with the trickier aspects of magnetotellurics and Euan Smith for help with the error analysis of the receiver functions.

Thank you to GNS Science for providing me with scholarship funding and magnetotelluric equipment. I would especially like to thank Hugh Bibby for arranging and helping with the field work necessary for this part of my thesis. Thank you also to Stephen Bannister who provided me with guidance in the early stages of my thesis.

I have used seismic data from the GeoNet network and would like to acknowledge the people behind this important information resource.

Thank you to all the organisations who supported this project by providing funding for the equipment and field costs, these include: The Lottery Grants Board, The Marsden Fund, Foundation for Research Science and Technology and Planet Earth Fund.

I would like to thank my friends and colleagues for their support, discussion and laughs along the way and for all those who helped with field work; Mark Henderson, Wanda Stratford, Sonja Greve, Matthieu Duclos, Stewart Bennie, Sandra Bourguignon, Sonja Greve, Erik Ewig and Anya Seward.

Lastly, I would like to thank my family and flatmates, especially my brother Greg who kept me going over the last stretch. 


\section{Contents}

$\begin{array}{ll}\text { Abstract } & \text { i }\end{array}$

Acknowledgements $\quad$ iii

1 Introduction 1

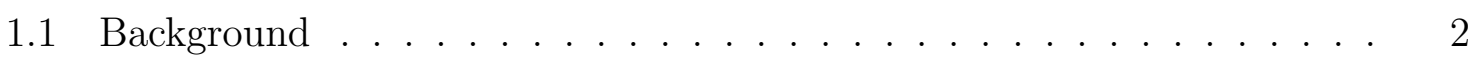

1.2 Tectonic setting . . . . . . . . . . . . . . . 4

1.2.1 The Central Volcanic Region . . . . . . . . . . . . . . 7

1.2.2 Northwestern North Island . . . . . . . . . . . . . . . . . . . . 7

1.2.3 South Wanganui Basin . . . . . . . . . . . . . . . . 7

2 Receiver functions $\quad 9$

2.1 Receiver function abstract . . . . . . . . . . . . . . . 9 9

2.2 The receiver function technique . . . . . . . . . . . . . . 9

2.3 Data Collection and Analysis . . . . . . . . . . . . . . 13

2.3.1 Earthquake event selection . . . . . . . . . . . . . . 15

2.3.2 Receiver Function Analysis . . . . . . . . . . . . . . . . . 16

2.4 Receiver function observations . . . . . . . . . . . . . . . . 23

2.4 .1 TRAP stations . . . . . . . . . . . . . . 23

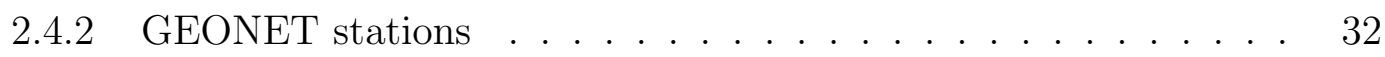

2.4.3 CNIPSE stations . . . . . . . . . . . . . . . . 37

2.5 Interpretation . . . . . . . . . . . . . . . . . . . 38

2.5.1 Piercing points . . . . . . . . . . . . . . . 38

2.5.2 Moho arrivals . . . . . . . . . . . . . . . . . . 40 


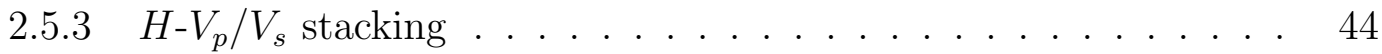

2.5.4 CCP gathers and depth migration . . . . . . . . . . 47

2.5.5 Dipping layers and anisotropy . . . . . . . . . . . 56

2.6 Receiver function summary . . . . . . . . . . . . . . . . . . . 57

3 Seismic Attenuation $\quad 61$

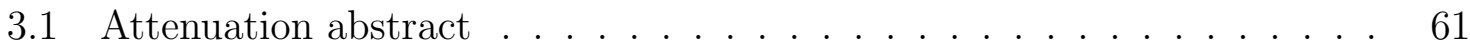

3.2 Seismic attenuation theory $\ldots \ldots \ldots \ldots \ldots$. . . . . . . . . 61

3.3 Data collection and analysis . . . . . . . . . . . . 65

3.3.1 Earthquake event selection . . . . . . . . . . . . . . . 66

3.3.2 Spectral decay method . . . . . . . . . . . . . . . 66

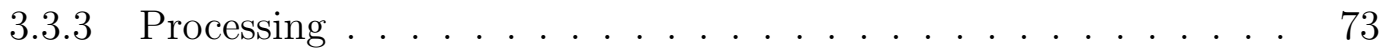

3.4 Attenuation results $\ldots \ldots \ldots \ldots \ldots \ldots \ldots \ldots \ldots$

3.4.1 CNIPSE data attenuation results $\ldots \ldots \ldots \ldots \ldots$

3.4 .2 TRAP data attenuation results $\ldots \ldots \ldots \ldots \ldots$

3.5 Interpretation $\ldots \ldots \ldots \ldots \ldots \ldots \ldots \ldots \ldots$

3.5.1 Temperature, melt, water and Q . . . . . . . . . 86

4 Magnetotellurics $\quad 89$

4.1 Magnetotellurics abstract . . . . . . . . . . . . . . . . . . 89

4.2 MT Theory . . . . . . . . . . . . . . . . . . . . . 89

4.2.1 Source Signal and Basic Principles _ . . . . . . . . . . . . . 89

$4.2 .2 \quad$ Skin $\operatorname{depth} \ldots \ldots \ldots \ldots \ldots$. . . . . . . . . . . . . . . . 92

4.2 .3 The impedance tensor $\ldots \ldots \ldots \ldots$. . . . . . . . . . 92

$4.3 \quad$ MT Data . . . . . . . . . . . . . . . . . . . . . 97

4.3 .1 Data acquisition . . . . . . . . . . . . . . . 97

4.3 .2 Processing . . . . . . . . . . . . . . . . . 100

4.3 .3 Magnetotelluric results . . . . . . . . . . . . . . . . . . . 101

$4.4 \quad$ MT modelling . . . . . . . . . . . . . . . . . . . . . . . . . . 111

4.4 .1 1D modelling . . . . . . . . . . . . . . . . 120 
4.4 .2 2D modelling . . . . . . . . . . . . . . . . . . 124

4.5 Interpretation . . . . . . . . . . . . . . . . 130

5 Discussion

135

5.1 Temperature, melt and water content . . . . . . . . . . . . 135

5.2 The Taranaki-Ruapehu Line . . . . . . . . . . . . . . . . 136

6 Conclusions 141

$\begin{array}{ll}\text { Appendices } & 155\end{array}$

$\begin{array}{lr}\text { A Station information } & 157\end{array}$

B Receiver Functions $\quad 159$

$\begin{array}{lll}\mathrm{C} & \text { Seismic Attenuation } & 169\end{array}$

$\begin{array}{ll}\text { D Magnetotellurics } & 171\end{array}$ 


\section{List of Figures}

1.1 North Island upper mantle inhomogeneity . . . . . . . . . . . . . . 3

1.2 Isostatic gravity map . . . . . . . . . . . . . . . . . 4

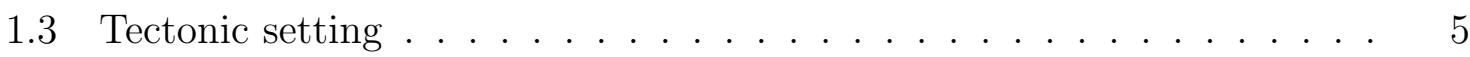

1.4 Back-arc regions of the North Island . . . . . . . . . . . . . 6

2.1 Moho reflection-transmission coefficients . . . . . . . . . . . . . . 10

2.2 Receiver function schematic . . . . . . . . . . . . . . . . . 11

2.3 Receiver function reference frame . . . . . . . . . . . . . . . . . 12

2.4 Station location . . . . . . . . . . . . . . . . . 14

2.5 Teleseismic events for TRAP array . . . . . . . . . . . . . 16

2.6 Teleseismic events for GEONET stations . . . . . . . . . . . . . . . 17

2.7 Teleseismic events for CNIPSE stations . . . . . . . . . . . . . . . . 18

2.8 Teleseismic event spectrogram . . . . . . . . . . . . . . . . . . . . . . . . 19

2.9 Teleseismic event spectrogram . . . . . . . . . . . . . . . . . 20

2.10 Prolate tapers . . . . . . . . . . . . . . . . . . . . 21

2.11 TUN receiver functions . . . . . . . . . . . . . . . . . . . . . 24

2.12 KAI receiver functions . . . . . . . . . . . . . . . . . . 26

2.13 LQT receiver functions for KAI . . . . . . . . . . . . . . 26

2.14 ERU receiver functions . . . . . . . . . . . . . . . . . . . . 27

2.15 LQT receiver functions for ERU . . . . . . . . . . . . . . . . . 27

2.16 RAE receiver functions . . . . . . . . . . . . . . . . . . . . . . . 29

2.17 LQT receiver functions for RAE . . . . . . . . . . . . . . . . . . . . . 29

2.18 PAP receiver functions . . . . . . . . . . . . . . . . 30 
2.19 LQT receiver functions for PAP . . . . . . . . . . . . . . 30

2.20 SUN receiver functions . . . . . . . . . . . . . . . . 31

2.21 WRR receiver functions . . . . . . . . . . . . . . . 31

2.22 HIZ receiver functions . . . . . . . . . . . . . . . . . 33

2.23 LQT receiver functions for HIZ . . . . . . . . . . . . . . . 33

2.24 VRZ receiver functions . . . . . . . . . . . . . . . . . 35

2.25 LQT receiver functions for VRZ . . . . . . . . . . . . . . . . 35

2.26 WAZ receiver functions . . . . . . . . . . . . . . . . . . . . 36

2.27 LQT receiver functions for WAZ . . . . . . . . . . . . . . . . 36

2.28 YUPC receiver functions . . . . . . . . . . . . . . . . 37

2.29 MILC receiver functions . . . . . . . . . . . . . . . . . . 38

2.30 Piercing points . . . . . . . . . . . . . . . . . 39

2.31 Expected delay times . . . . . . . . . . . . . . . . 43

$2.32 H-V_{p} / V_{s}$ stack for station $\mathrm{KAI} \ldots \ldots \ldots \ldots$

$2.33 H-V_{p} / V_{s}$ stack for station $\mathrm{VRZ} \ldots \ldots \ldots \ldots \ldots$

$2.34 \mathrm{~S}-\mathrm{N}$ CCP stack section . . . . . . . . . . . . . . . 51

2.35 NW-SE CCP stack section . . . . . . . . . . . . . . . 52

2.36 Ray parameter stacks for TRAP sites . . . . . . . . . . . . . 54

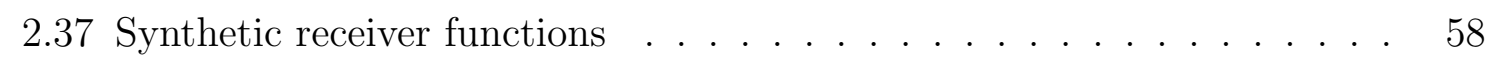

2.38 Velocity model from receiver functions . . . . . . . . . . . . . . 59

3.1 Displacement amplitude decay for a constant $Q$ value . . . . . . . . . 62

3.2 Melt dissipation peak . . . . . . . . . . . . . . . 64

3.3 Station locations ............................ 67

3.4 Local earthquake locations for the TRAP array . . . . . . . . . . 68

3.5 Earthquake depth profile for the TRAP array . . . . . . . . . . . 69

3.6 Local earthquake locations for the CNIPSE array . . . . . . . . . . 70

3.7 Instrument responses f . . . . . . . . . . . . . . . . . . . . . 72

3.8 ORION-EARSS huddle test . . . . . . . . . . . . . . . 73 
3.9 EARSS-Reftek huddle test . . . . . . . . . . . . . . . . . . . . 74

3.10 Attenuated S-wave . . . . . . . . . . . . . . . . . . . . 74

3.11 P-wave data windows . . . . . . . . . . . . . . . . . 75

3.12 Typical attenuation data fit . . . . . . . . . . . . . . . 77

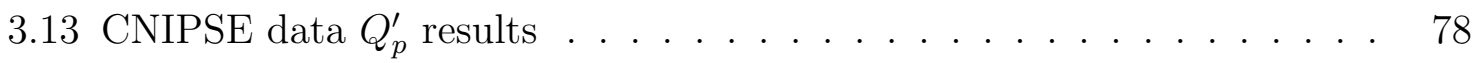

3.14 Earthquake depths vs. $Q_{p}^{\prime} \ldots \ldots \ldots \ldots \ldots \ldots$

3.15 Northern TRAP stations $Q_{P}^{\prime}$ results . . . . . . . . . . . . . 81

3.16 Southern TRAP stations $Q_{P}^{\prime}$ results . . . . . . . . . . . . . . . . 82

3.17 Mean attenuation for TRAP stations . . . . . . . . . . . . . 83

3.18 Frequency dependence . . . . . . . . . . . . . . . 85

3.19 Homologous temperature vs $Q_{s} \ldots \ldots \ldots \ldots$. . . . . . . . . 87

4.1 Magnetic field variations . . . . . . . . . . . . . . . . . . . . . . . 90

4.2 MT signal . . . . . . . . . . . . . . . . . . . . . . . . . . . 91

4.3 1D layered earth . . . . . . . . . . . . . . . . . . 94

4.4 Typical MT station layout . . . . . . . . . . . . . . . . . . 97

4.5 Station location . . . . . . . . . . . . . . . . . . . . . . . . 98

4.6 Magnetic storm activity . . . . . . . . . . . . . . . . . . . . . . . 99

4.7 Dimensionality station $001 \ldots \ldots \ldots$. . . . . . . . . . . . 103

4.8 Dimensionality station $002 \ldots \ldots$. . . . . . . . . . . . 104

4.9 Dimensionality station $003 \ldots \ldots \ldots \ldots$

4.10 Dimensionality station $004 \ldots \ldots \ldots$. . . . . . . . . . . 106

4.11 Dimensionality station $005 \ldots \ldots \ldots$. . . . . . . . . . . . 107

4.12 Dimensionality station $006 \ldots \ldots \ldots$. . . . . . . . . . . 108

4.13 Phase tensor principal axis . . . . . . . . . . . . . . . . . . 109

4.14 MT station $001 \ldots \ldots \ldots \ldots$. . . . . . . . . . . . . . . 112

4.15 MT station $002 \ldots \ldots \ldots$. . . . . . . . . . . . . . . . 113

4.16 MT station $003 \ldots \ldots$. . . . . . . . . . . . . . . . . 114

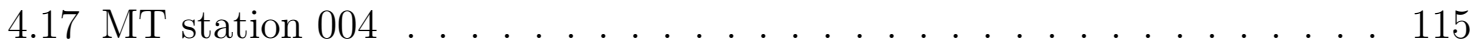


4.18 MT station $005 \ldots \ldots \ldots \ldots$

4.19 MT station $006 \ldots \ldots \ldots \ldots$. . . . . . . . . . . . . . . . . . . . . . . . . . . .

4.20 Apparent resistivity pseudo-sections . . . . . . . . . . . . . . 118

4.21 Phase pseudo-sections . . . . . . . . . . . . . . . . . . . 119

$4.221 \mathrm{D}$ models for stations $001-003 \ldots \ldots$. . . . . . . . . . . . 121

$4.231 \mathrm{D}$ models for stations $004-006 \ldots \ldots$. . . . . . . . . . 122

4.24 1D Occam model sediments TM mode . . . . . . . . . . . . . 123

4.25 Input mesh for MT 2D-inversion . . . . . . . . . . . . . . 126

4.26 Shallow $2 \mathrm{D}$ resistivity structure . . . . . . . . . . . . . 128

4.27 Best fit 2D resistivity model . . . . . . . . . . . . . . . . . . 129

$4.282 \mathrm{D}$ resistivity model data fit to stations $001-003 \ldots \ldots$. . . . . . 131

$4.292 \mathrm{D}$ resistivity model data fit to stations $004-006 \ldots \ldots$. . . . . . . 132

4.30 Basement depth . . . . . . . . . . . . . . . . . 133

5.1 Taranaki-Ruapehu Line . . . . . . . . . . . . . . . . . 138

D.1 2D inversion model $1 \ldots \ldots$. . . . . . . . . . . . . 172

D.2 Fit to inversion model 1 for stations $001-003 \ldots \ldots$. . . . . . . . 173

D.3 Fit to inversion model 1 for stations $004-006$. . . . . . . . . . . . . 174

D.4 2D inversion model $2 \ldots \ldots \ldots \ldots$. . . . . . . . . . . . . . . . . . . . .

D.5 Fit to inversion model 2 for stations $001-003 \ldots \ldots . \ldots . \ldots 176$

D.6 Fit to inversion model 2 for stations $004-006 \ldots \ldots$. . . . . . . 177

D.7 2D inversion model $3 \ldots \ldots \ldots \ldots$. . . . . . . . . . . . 178

D.8 Fit to inversion model 3 for stations $001-003 \ldots \ldots$. . . . . . . . 179

D.9 Fit to inversion model 3 for stations $004-006 \ldots \ldots$. . . . . . . 180

D.10 2D inversion model $4 \ldots \ldots$. . . . . . . . . . . . 181

D.11 Fit to inversion model 4 for stations $001-003 \ldots \ldots$. . . . . . . . 182

D.12 Fit to inversion model 4 for stations $004-006$. . . . . . . . . . . . 183 


\section{List of Tables}

2.1 Sediment depth estimates below stations . . . . . . . . . . . . . . 13

2.2 New Zealand velocity models $[$ Maunder 2002] . . . . . . . . . . . 55

4.1 MT station static shifts . . . . . . . . . . . . . . . . 128

A.1 Seismic station location and instrumentation. . . . . . . . . . . 157

A.2 Seismic station deployment periods for TRAP and CNIPSE arrays. . . 158

A.3 Magnetotelluric station locations f . . . . . . . . . . . 158

B.1 TRAP receiver function events . . . . . . . . . . . . . . . 159

B.2 GEONET receiver function events . . . . . . . . . . . . . . . . . . 161

B.3 CNIPSE receiver function events . . . . . . . . . . . . . . 166

C.1 TRAP mean $1000 / Q_{p}^{\prime}$ and normalisation factors . . . . . . . . . 169

C.2 Constants for dissipation background, $Q_{B}^{-1} \ldots \ldots \ldots$. . . . . 170

C.3 Constants for dissipation peak, $Q_{M P}^{-1} \ldots \ldots \ldots \ldots \ldots$

D.1 Minimum and maximum skin depths . . . . . . . . . . . . . . . 171 


\section{Chapter 1}

\section{INTRODUCTION}

Back-arc regions vary in deformation style and structure from compressional regions with thickened crust to regions of active extensional rifting [Uyeda 1982, Uyeda and Kanamori 1979]. This variability of back-arc regions has important implications for both plate and continental tectonics. Compressional back-arcs are linked to subduction zones with strong plate coupling that experience great earthquakes (e.g. Chile). Extension close to a convergent plate boundary seems paradoxical, distinct rift structures have formed by extension in back-arc settings (e.g. Lau-Havre trough). High heat-flow, high seismic attenuation and low Pn velocities are all observed in back-arc regions [Currie and Hyndman 2006, Uyeda 1977] and basins are often elevated with respect to their surroundings [Sclater 1972]. These observations point to the presence of hot buoyant mantle inhomogeneities beneath these regions that could help account for volcanism at their peripheries. It is important to understand these regions as they are not only an enigmatic feature of global tectonics but are linked to natural hazards and resources that have a high societal impact.

The western North Island of New Zealand lies within the back-arc region of the Hikurangi subduction zone. This back-arc region has two points of difference that make it a favourable location to study back-arc structure and processes. Firstly, the back-arc regions of western North Island can be divided into a northern region of extension and a southern region of compression. Secondly, the region of extension extends onshore into continental New Zealand, making it easily accessible and providing a rare opportunity to observe the manifestations of back-arc spreading in continental lithosphere.

This thesis is directed to exploring the back-arc region of North Island New Zealand with a particular focus on the E-W boundary that runs nearly perpendicular to the present plate boundary. This boundary marks and abrupt change from extension to compression and is associated with a fundamental change in geophysical properties which indicate the presence of a low-density mantle anomaly below northwestern North Island. Receiver functions are used in chapter 2 to constrain crustal thickness either side of the boundary. Seismic attenuation of the back-arc mantle is investigated in chapter 3. Attenuation is then used to provide constraints on mantle temperature and 
melt content. In chapter 4 magnetotelluric data is used to examine electrical resistivity of the crust and upper mantle across the boundary.

\subsection{Background}

An upper mantle inhomogeneity, manifesting as a region of high seismic attenuation, was recognised below the Northwestern North Island (NWNI) and the Central Volcanic Region (CVR) in the early 1970's [Hatherton 1970, Mooney 1970] (figure 1.1). It was also recognised that the lateral extent of this anomaly coincided with a region of positive isostatic gravity anomalies (figure 1.2) and with distributed volcanism. The southern extent of the anomaly runs approximately E-W between Mt Taranaki and Mt Ruapehu. This boundary will henceforth be referred to as the Taranaki-Ruapehu Line (TRL).

Subsequent studies revealed that the attenuating zone is associated with changes in deformation style, Pn velocities, heat flow and crustal thickness. Geodetically determined strain rates show that there is a change from trench perpendicular shortening south of the TRL to either zero strain rates or trench perpendicular extension north of the TRL [Reilly 1990]. Haines [1979] showed that the CVR correlates with a region of extremely low upper mantle P-wave velocities $(\sim 7.4 \mathrm{~km} / \mathrm{s})$. In the same study low Pn velocities were also observed below the NWNI $(\sim 7.9 \mathrm{~km} / \mathrm{s})$. More recently the results of active source studies confirm the low P-wave velocities below both the CVR and the NWNI [Harrison and White 2004, Stern et al. 1987, Stratford and Stern 2004].

The crustal thickness was estimated north of the TRL from a refraction study [Stern et al. 1987] and south of the TRL from a deep seismic reflection profile [Stern and Davey 1990]. North of the line crust is thin $(25 \mathrm{~km})$ and to the south, below the south Wanganui basin the crust is thick $(\sim 40 \mathrm{~km})$. The steep gravity gradient at the TRL was attributed to an edge-effect caused by a $10 \mathrm{~km}$ step in crustal thickness which is isostatically compensated for by a low density mantle north of the TRL [Stern et al. 1987]. Extremely high heat output of about $4.3 \mathrm{GW}$ in the CVR has long been recognised [Bibby et al. 1995, Stern 1987], which when averaged over the area of geothermal activity suggests an effective heat flow of about $840 \mathrm{mWm}^{-2}[$ Studt and Thompson 1969]. This heat flux has been exploited for geothermal power production for several decades. The NWNI is also associated with elevated heat flow $\left(86 \mathrm{mWm}^{-2}\right)$ compared with that of the rest of the North Island $\left(40-60 \mathrm{mWm}^{-2}\right)$ [Pandey 1981a;b]. A hot low-density mantle would help to explain the above observations and would provide the buoyancy force required to keep the thin crust of the CVR $(\sim 20 \mathrm{~km})$ and NWNI (25-28 km) at its present elevation [Horspool et al. 2006, Pulford and Stern 2004, Stratford and Stern 2006]. The final unusual observation is an E-W line of earthquakes between Mt Taranaki and Mt Ruapehu at depths between 25-40 km [Reyners 1978]. 


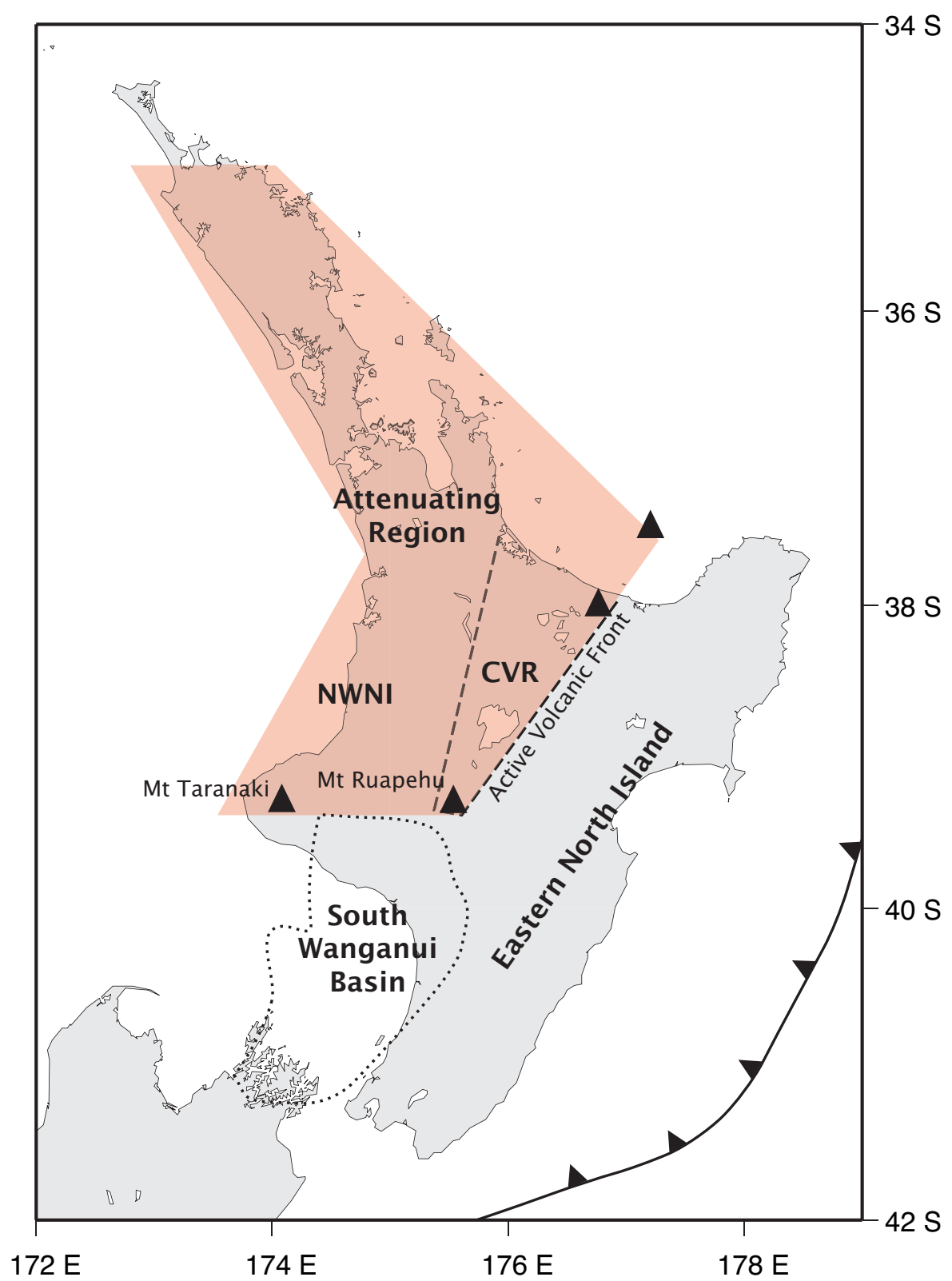

Figure 1.1: North Island upper mantle inhomogeneity. The pink shaded region indicates the approximate extent of the attenuating mantle anomaly [Mooney 1970]. The region encompasses the Central Volcanic Region (CVR) and the Northwestern North Island (NWNI). The dashed line shows the boundary of the CVR, which is the onshore region associated with active back-arc extension. Active andesite volcanoes are shown as black triangles. The dotted line encompasses the South Wanganui Basin. 


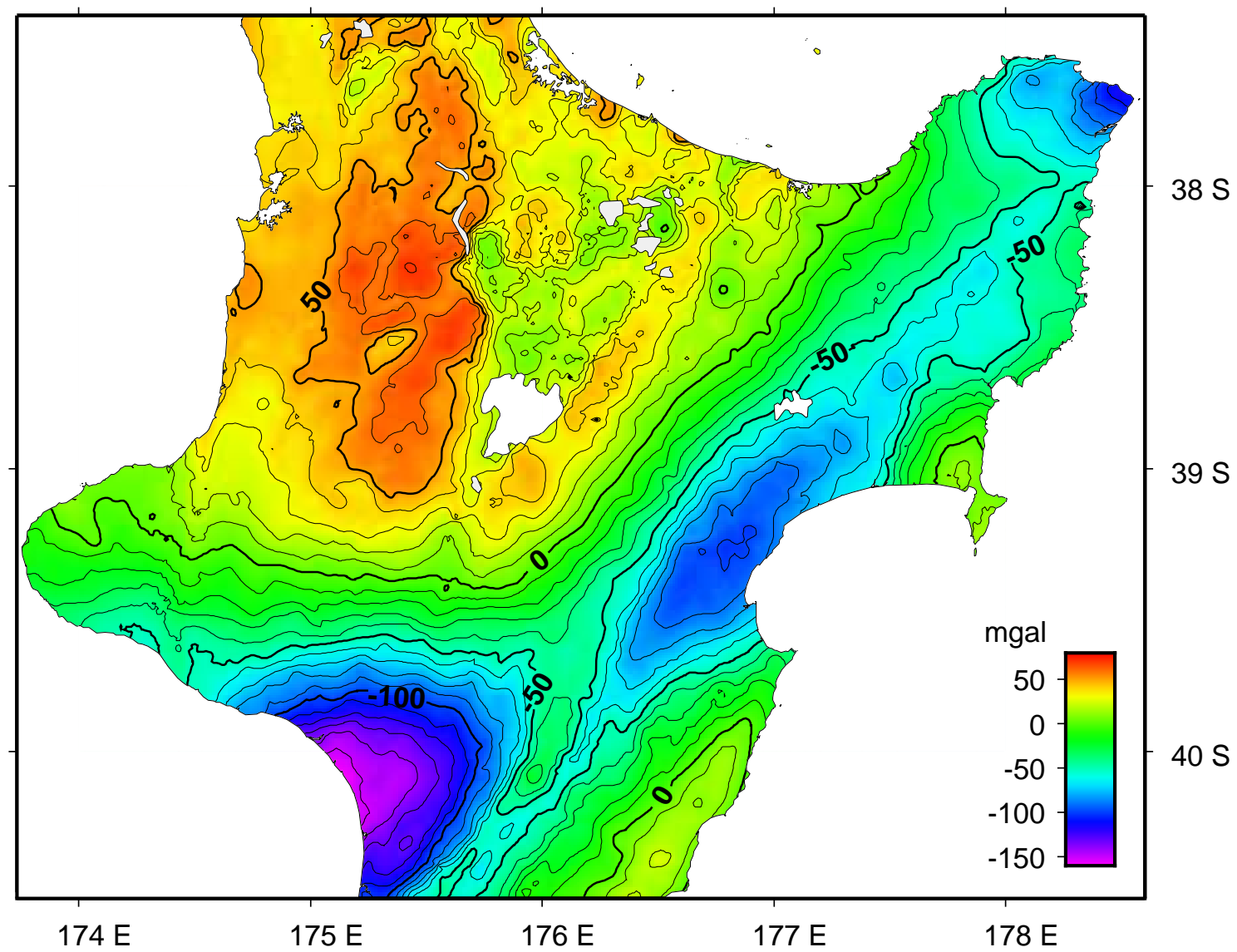

Figure 1.2: Isostatic gravity map of the northwestern North Island [Reilly et al. 1977].

These earthquakes have been attributed to changes in mantle flow and fluid content at a step in crustal thickness [Reyners et al. 2006] or deviatoric stresses linked to a sudden change in crustal thickness [Stern et al. 1987].

\subsection{Tectonic setting}

New Zealand lies across the Australian-Pacific plate boundary at the junction between two opposing subduction regimes. To the north the Pacific plate subducts westward below the Australian plate and to the south the Australian plate subducts eastward below the Pacific plate (figure 1.3). New Zealand forms the transition between these two zones. The subduction regime in the north is continued southward with the Pacific plate subducting obliquely below the North Island of New Zealand at the Hikurangi trench. The western North Island forms the back-arc region of this subduction zone. The basic elements of this plate boundary system were established by 21 Ma [King 2000]. The western North Island has undergone $\sim 70 \mathrm{~km}$ of Miocene shortening, forming a broad compressional foreland basin west of the Taranaki fault zone [Stern and Davey 1990]. By 5 Ma shortening north of the TRL had ceased and was followed by the opening of the CVR [King 2000] and $\sim 2.5 \mathrm{~km}$ of broad-wavelength rock uplift [Pulford and Stern 2004]. Shortening continues to the present day south of the TRL 


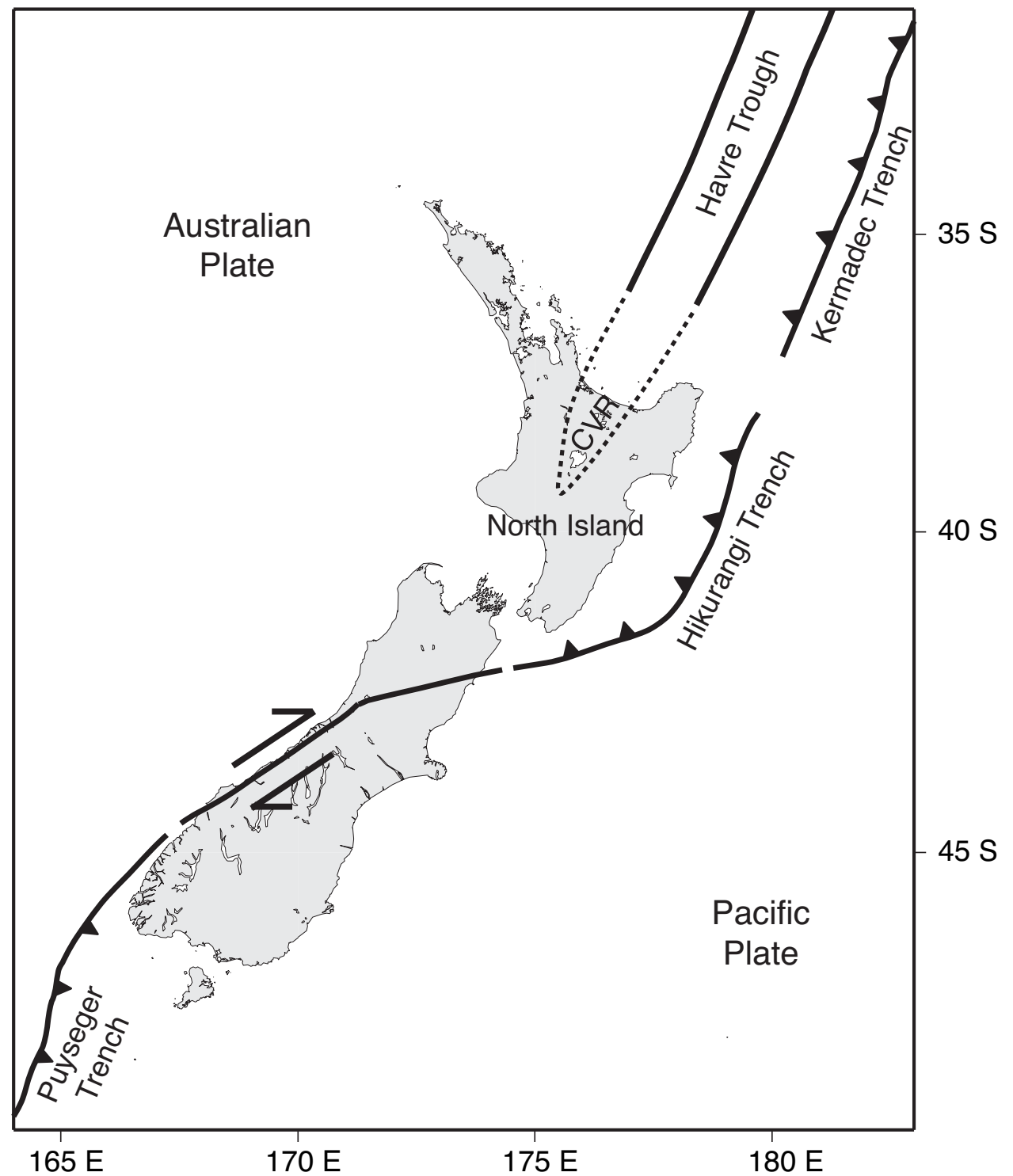

Figure 1.3: New Zealand tectonic setting. 
[Reilly 1990, Wallace et al. 2004] and is accompanied by the subsidence of the South Wanganui basin [Anderton 1981].

The back-arc region of the North Island can be split into three distinct geographical zones; Central Volcanic Region (CVR), Northwestern North Island (NWNI) and the South Wanganui Basin (figure 1.4).

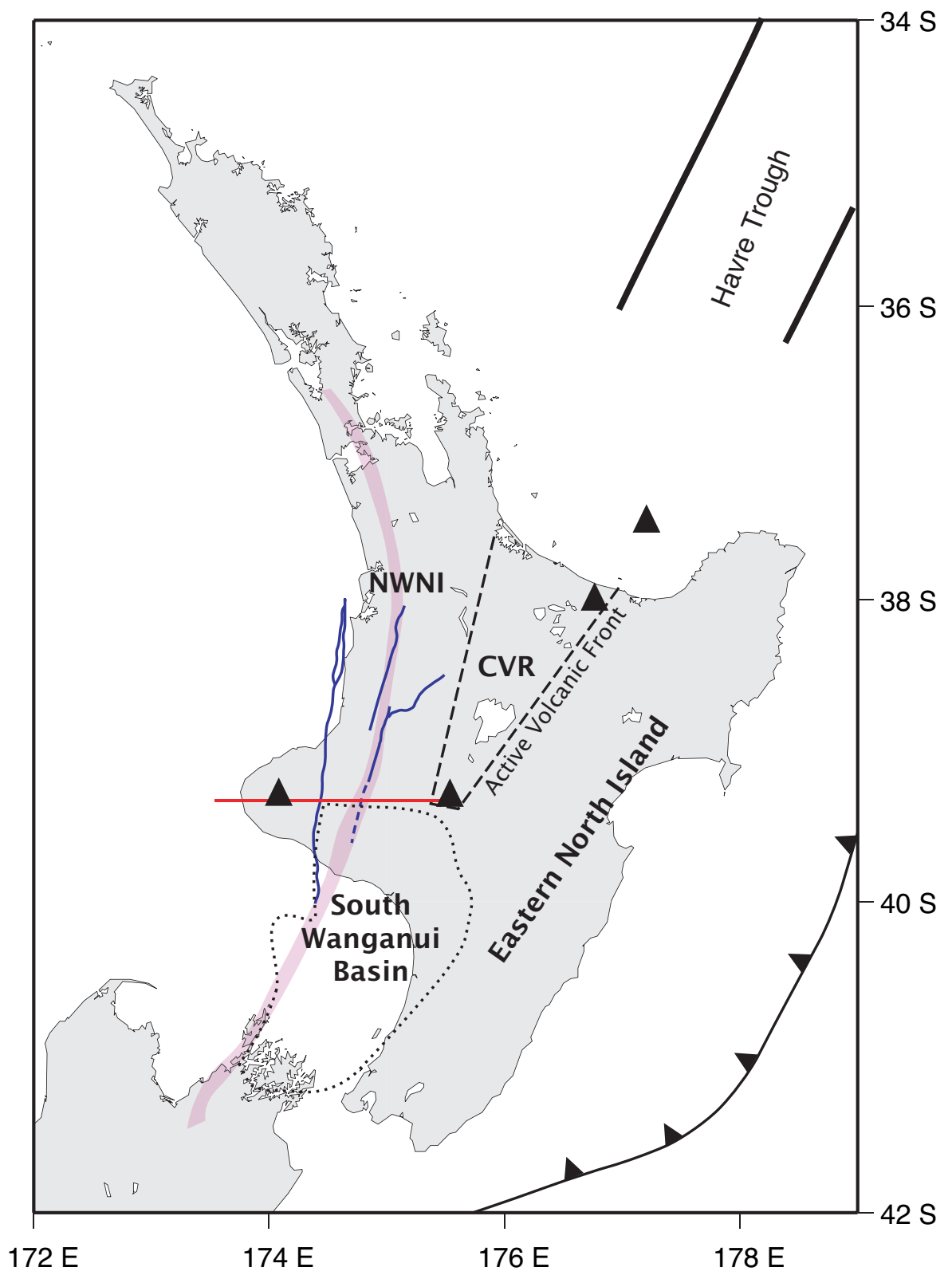

Figure 1.4: Back-arc regions of the North Island. The Taranaki-Ruapehu line is shown in red. The dotted line indicated the boundaries of the South Wanganui Basin. Boundaries of the CVR are indicated by the dashed line. Major faults of the Taranaki fault zone are shown as solid blue lines. The junction magnetic anomaly (Maitai Terrane) is shown in pink. Active andesite volcanoes are shown as black triangles. 


\subsubsection{The Central Volcanic Region}

Subduction north of New Zealand is associated with back-arc extension at the Havre Trough (figure 1.3). The CVR is the apparent continuation of the Lau-Havre Trough into continental New Zealand [Karig 1970]. The CVR is undergoing active extension of between 5-15 mm/yr [Wallace et al. 2004]. The extent of the CVR is most clearly defined by a wedge-shaped low in the residual gravity anomaly [Stern 1979].

The surface geology of the CVR is dominated by volcanic deposits (1.8-2.7 km thick) [Stern and Davey 1987]. Basement rocks are of unknown composition. Greywacke ranges flank the eastern margin of the CVR but deep bore holes of up to $3 \mathrm{~km}$ in depth in the centre of the region all terminate in rocks of volcanic or plutonic origin [Stern 1986, Stern and Davey 1987]. Seismicity within the CVR is confined to the near surface (most shallow earthquakes are at depths $<6 \mathrm{~km}$ [Bryan et al. 1999]) and the subducting slab [Ansell and Bannister 1996]. The CVR is associated with crustal thinning [Stratford and Stern 2004], high heat output of 4.3 GW [Bibby et al. 1995] and high seismic attenuation [Mooney 1970, Satake and Hashida 1989].

\subsubsection{Northwestern North Island}

The northwestern North Island is bounded by the CVR to the east and the TRL to the south (figure 1.4). This region underwent $\sim 70 \mathrm{~km}$ of Miocene shortening. The surface geology is dominated by a series of uplifted sedimentary basins. These basins have a migrated southward with time [Kamp 1999]. There is also a series of N-S trending crustal scale faults known as the Taranaki fault zone [Stern and Davey 1990]. This $\sim 80 \mathrm{~km}$ wide zone is inferred to be the region where most of the crustal shortening occurred during the Miocene and is also the location of a Permian crustal suture known as the Junction Magnetic Anomaly (also known as the Maitai Terrane) [Hunt 1978].

The northwestern North Island is associated with high heat flow $\left(86 \mathrm{mWm}^{-2}\right)[$ Pandey 1981a], thin crust (25-28 km) [Horspool et al. 2006, Stern et al. 1987] and high seismic attenuation [Mooney 1970, Satake and Hashida 1989].

\subsubsection{South Wanganui Basin}

The South Wanganui basin lies to the south the TRL (figure 1.4); it is bounded to the east by greywacke ranges and to the west a submarine basement high [Anderton 1981]. This basin formed by progressive subsidence and apparent crustal down-warping to the south at around the same time as the uplift of the North Island [Anderton 1981, Stern et al. 1992]. About $\sim 4 \mathrm{~km}$ of Plio-Pleistocene marine sediment fill is inferred from drill hole data and seismic reflection profiles [Anderton 1981]. The present day depocentre lies offshore but appears to still be migrating southward [Stern et al. 1992]. Unusually deep crustal earthquakes with mixed focal mechanisms occur beneath the 
basin [Garrick and Gibowicz 1983]. This region is associated with normal heat flow $\left(\sim 60 \mathrm{mWm}^{-2}\right)$, thick down-warped crust [Stern and Davey 1990], a deep low in the isostatic gravity anomaly [Reilly et al. 1977] and low seismic attenuation [Mooney 1970, Satake and Hashida 1989]. 


\section{Chapter 2}

\section{RECEIVER FUNCTIONS}

\subsection{Receiver function abstract}

Receiver function analysis reveals a $7 \mathrm{~km}$ step-like change in crustal thickness across the Taranaki-Ruapehu Line (TRL). North of the TRL receiver functions show a strong Ps conversion at $25 \pm 1.5 \mathrm{~km}$ depth, which is interpreted as a shallow Moho. At the TRL the Moho Ps conversion deepens to $\sim 32 \mathrm{~km}$ depth and weakens. This change in crustal thickness occurs over a lateral distance of $\sim 20 \mathrm{~km}$. South of the TRL the Moho dips gently to the south. Also imaged in the receiver functions is a mantle boundary at $\sim 65 \mathrm{~km}$ below northwestern North Island.

\subsection{The receiver function technique}

Receiver functions have long been recognised as an important tool for imaging crust and upper mantle seismic discontinuities such as the crust-mantle boundary [Langston 1979, Owens and Zandt 1985]. If the Taranaki-Ruapehu Line is manifested as a change in crustal thickness, receiver functions should be able to image this.

The receiver function technique uses the coda of near vertically travelling body waves recorded on three component seismographs to gain information about the earth below the seismograph. The underlying assumption is that the coda of the body wave is formed by mode conversions and reverberations of the body wave at seismic boundaries. Here teleseismic $\mathrm{P}$ waves are used to calculate receiver functions and the following discussion will therefore be restricted to $\mathrm{P}$ wave receiver functions.

A P wave incident on a seismic boundary will produce transmitted, reflected and converted phases that propagate as both $\mathrm{P}$ and $\mathrm{S}$ waves. The relative amplitudes of the resulting waves depends on the impedence contrasts across the boundary and the incidence angle of the incoming wave (figure2.1).

$\mathrm{P}$ waves traveling from an earthquake to the surface of the earth will encounter seismic 
(a)

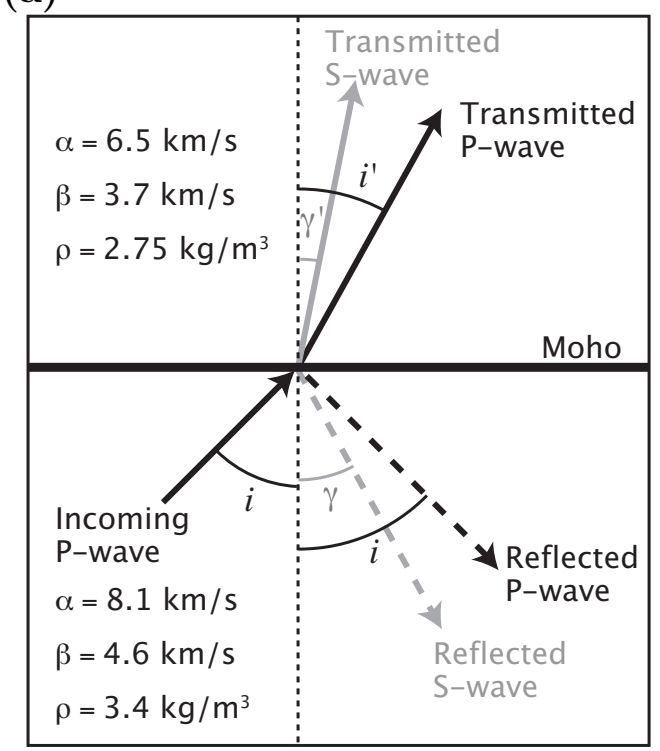

Reflection and refraction coefficients

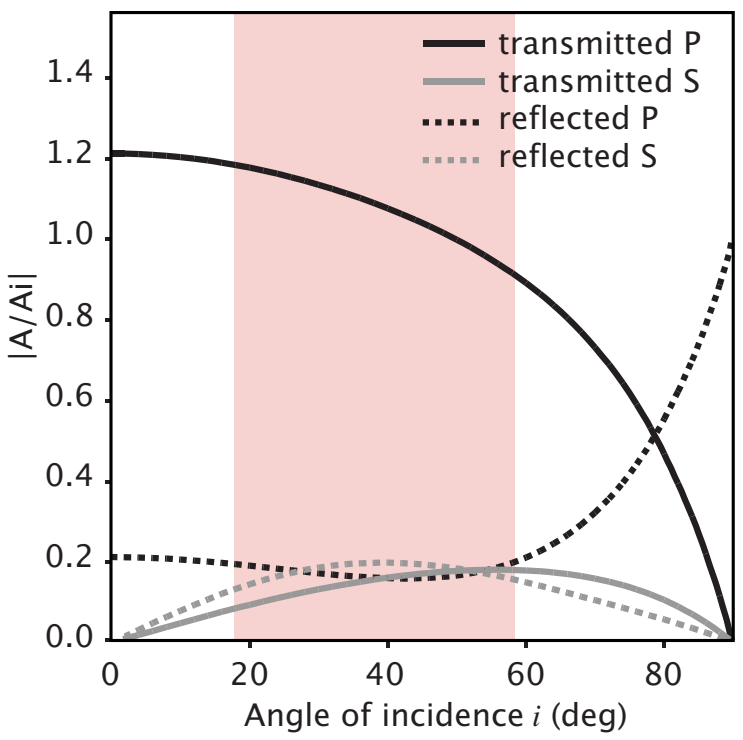

Figure 2.1: Reflection-transmission coefficients for the crust-mantle boundary of the New Zealand standard velocity model. (a) shows a schematic of energy partitioning of a $\mathrm{P}$ wave incident on a seismic boundary where $\alpha$ is the $\mathrm{P}$-wave velocity, $\beta$ is the $\mathrm{S}$-wave velocity, $\rho$ is the density, $i$ is the incidence angle and angle of reflection of the $\mathrm{P}$ wave, $\gamma$ is the angle of reflection of the $\mathrm{S}$ wave, $i^{\prime}$ and $\gamma^{\prime}$ are the angles of refraction for the $\mathrm{P}$ and $\mathrm{S}$ waves respectively. (b) shows the relative amplitudes of the transmitted and reflected phases in (a) calculated using Zoeppritz's equations. Amplitudes (A) are normalised to the incident $\mathrm{P}$ wave amplitude $(\mathrm{Ai})$. The pink shaded region indicates the range of incidence angles for this study.

boundaries on their way. Where the incidence angle of the incoming $\mathrm{P}$ wave is less than $90^{\circ}$, part of the $\mathrm{P}$ wave will be transmitted as an $\mathrm{S}$ wave $(\mathrm{Ps})$. The converted $\mathrm{S}$ wave travels at a lower velocity than the $\mathrm{P}$ wave and therefore arrives at the receiver after the transmitted $\mathrm{P}$ wave $(\mathrm{Pp})$. Assuming the incident $\mathrm{P}$ waves are plane waves, the delay time between the Pp and the converted Ps phase $\left(t_{P s}\right)$ can be related to the depth of the conversion point using equation 2.2.1 [Gurrola et al. 1994].

$$
t_{P s}=H\left(\sqrt{\frac{1}{V_{s}^{2}}-p^{2}}-\sqrt{\frac{1}{V_{p}^{2}}-p^{2}}\right)
$$

where $H$ is the depth to the conversion boundary, $V_{s}$ is the $\mathrm{S}$ wave velocity between the conversion point and the receiver, $V_{p}$ is the $\mathrm{P}$ wave velocity and $p$ is the horizontal slowness or ray parameter $(p=d T / d \Delta$ where $T$ is the travel time and $\Delta$ is the angular distance for a station event pair).

In the case of teleseismic events, where the incoming $\mathrm{P}$ wave has a near vertical angle of incidence (earthquakes with epicentral distances between $30-110^{\circ}$ ), and horizontal seismic boundaries the $\mathrm{P}$ wave particle motion recorded at a receiver is mostly in the 

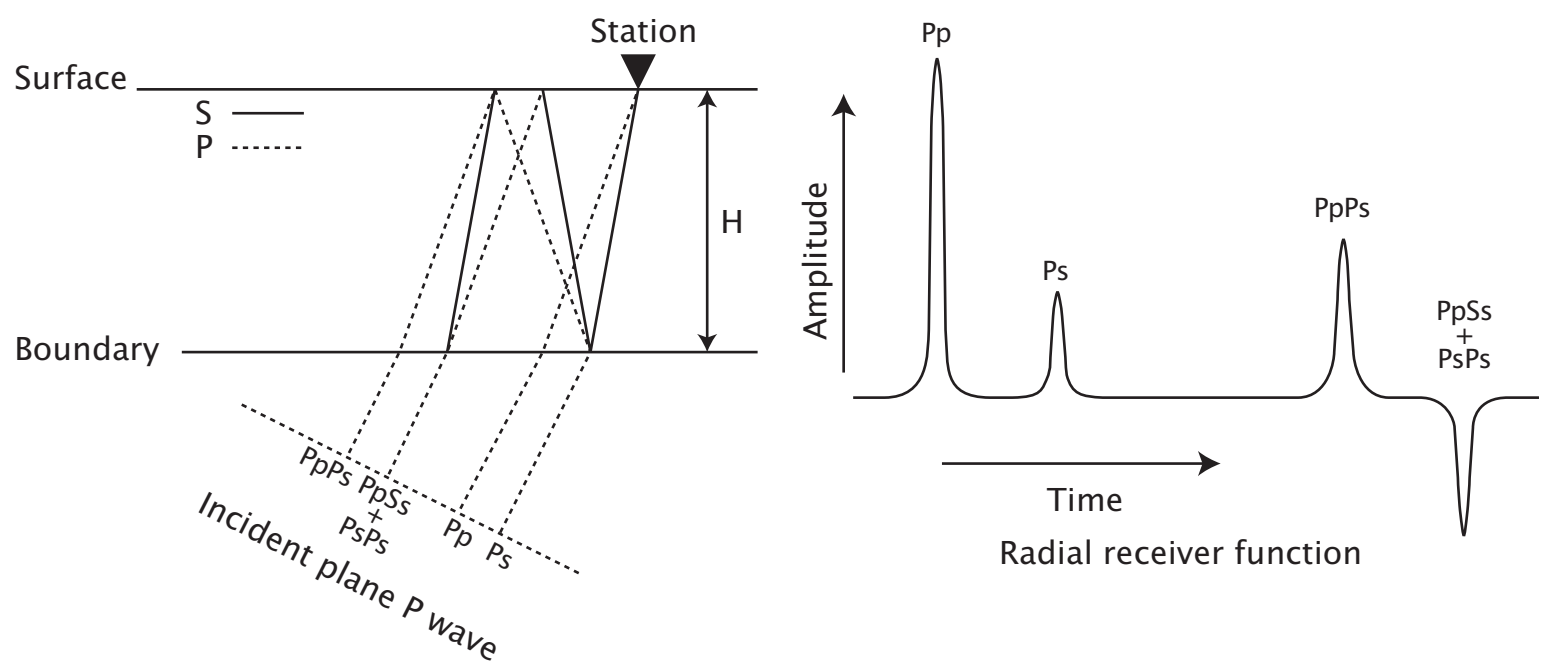

Figure 2.2: Receiver function schematic. Phases are named according to their paths. Except for the first letter, lower case letters denote up-going travel paths and upper case letters denote down-going travel paths. The left figure shows a raypath schematic for 5 different phases (Pp, Ps, PpPs, PsPs and PpSs) and the right figure shows an idealised receiver function for these phases.

vertical direction and the converted $\mathrm{S}$ wave particle motion is almost entirely in the radial direction (figure $2.1 \mathrm{~b}$ ).

The amplitude of the converted $\mathrm{S}$ waves will be small compared to the transmitted $\mathrm{P}$ wave (figure 2.1b). It is therefore often difficult to identify the converted phases in the raw data. To enhance the converted phases the receiver function technique uses the vertical component as an estimate of the waveform expected from the converted waves. The vertical component can be used to deconvolve the horizontal components, isolating converted phases from source and instrument responses (figure 2.2). In addition to the Ps conversion, multiples from the boundary may also be isolated in the receiver functions (e.g. PpPs, PpSs and PsPs figure 2.2). These can also be related to the depth of the boundary using equations 2.2.2 and 2.2.3.

$$
\begin{aligned}
& t_{P p P s}=H\left(\sqrt{\frac{1}{V_{s}^{2}}-p^{2}}+\sqrt{\frac{1}{V_{p}^{2}}-p^{2}}\right) \\
& t_{P s P s+P p S s}=2 H \sqrt{\frac{1}{V_{s}^{2}}-p^{2}}
\end{aligned}
$$

\section{LQT reference frame}

Receiver functions are normally calculated using radial and vertical components of a seismogram (ZRT reference frame), but the resulting receiver functions can be dominated by the presence of high amplitude Pp arrivals and $\mathrm{P}$ multiples. By rotating the 
radial and vertical components into a reference frame with one component along the propagation direction of the $\mathrm{P}$ wave (LQT figure 2.3) before receiver function analysis, $\mathrm{P}$ arrivals can be removed from the receiver function. This reference frame is particularly useful where regional earthquakes are used in the receiver function analysis since $\mathrm{P}$ waves from these events will tend to have a larger radial component.

\section{surface}

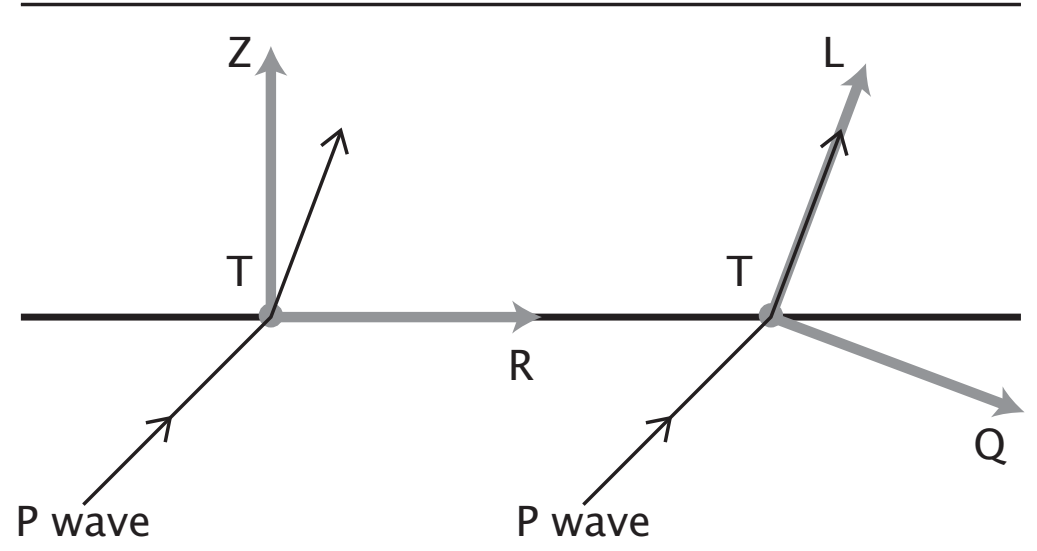

Figure 2.3: Receiver function reference frame. The ZRT reference frame is illustrated on the left. In this reference frame the horizontal seismogram components are rotated so that the $\mathrm{R}$ component is aligned along the station-event back azimuth and $\mathrm{T}$ is aligned orthogonally to R. LQT reference frame is shown on the right. In this reference frame the $\mathrm{Z}$ and $\mathrm{R}$ components are rotated so that the $\mathrm{L}$ component is aligned with the $\mathrm{P}$ wave propagation.

In order to rotate the reference frame correctly either the $\mathrm{P}$ wave velocity structure below the receiver and the ray parameter for the station-event pair or the angle of the $\mathrm{P}$ wave at the receiver need to be known. For data with little noise the $\mathrm{Z}$ and $\mathrm{R}$ components can be rotated to maximise the energy of the $\mathrm{P}$ arrival on the $\mathrm{L}$ component. Where this is not possible, velocity models must be used to estimate the degree of rotation required.

\section{Dipping layers and anisotropy}

For a horizontally layered isotropic earth the Pp and Ps arrivals will have a particle motion entirely in the radial plane, however where there are dipping layers or anisotropy there may be significant motion out of plane. Energy on the transverse receiver function is an indication that there are either dipping layers or anisotropy below the station. Details of the effects of both dipping layers and anisotropy on receiver functions can be found in Jones and Phinney [1998] and Savage [1998]. 


\subsection{Data Collection and Analysis}

Teleseismic-regional data from 12 seismic stations were used for receiver function analysis; 7 stations of the Taranaki-Ruapehu Attenuation Project (TRAP), 2 stations from the western end of the Central North Island Passive Seismic Experiment (CNIPSE) and three stations that belong to the New Zealand national network (GEONET) (figure 2.4). All stations are located west of the Central Volcanic Region and east of the Taranaki fault. The TRAP stations are all short period instruments (1 Hz seismometers) with a sampling rate of $100 \mathrm{~Hz}$. The CNIPSE stations are both broadband instruments, YUPC has a sampling rate of $100 \mathrm{~Hz}$ and MILC has a sampling rate of $50 \mathrm{~Hz}$. The GEONET stations are all broadband instruments with a $100 \mathrm{~Hz}$ sampling rate.

All TRAP stations and station WAZ sit on the sediments of the Wanganui basin. Sediment depths in general increase from north to south. Several decades of oil exploration in the region allow an assessment of sediments depths below each station to be made. A basement contour map has been constructed using drill hole data and seismic reflection lines [e.g. Gerrard 1971, St John 1964, Watson and Allen 1964] (figure 2.4). Combining this with station elevations gives a sediment thickness below each station (table 2.1).

Table 2.1: Sediment depth estimates below stations

\begin{tabular}{|c|c|c|l|}
\hline Station & $\begin{array}{c}\text { Elevation } \\
(\mathbf{m})\end{array}$ & $\begin{array}{c}\text { Sediment } \\
\text { thickness } \mathbf{( m )}\end{array}$ & $\begin{array}{l}\text { Closest basement } \\
\text { depth data points }\end{array}$ \\
\hline TUN & 390 & 390 & Kaitieke-1 \\
KAI & 680 & 1100 & $\begin{array}{l}\text { Kaitieke-1 } \\
\text { Tuapapakura-1 } \\
\end{array}$ \\
& & & Koporongo-1 \\
Koporongo-1 \\
ERU & 698 & 1700 & Seismic refraction line \\
RAE & 582 & 2300 & Seismic reflection line \\
PAP & 364 & 2100 & Seismic reflection line \\
SUN & 350 & 2400 & Parikino-1 \\
WRR & 70 & 2300 & NZ geological map \\
MILC & 290 & 0 & NZ geological map \\
YUPC & 562 & 0 & NZ geological map \\
HIZ & 237 & 0 & Seismic refection line \\
VRZ & 166 & 1300 & Parikino-1 \\
WAZ & 382 & 2600 & Seismic reflection line \\
\hline
\end{tabular}

Seismic refraction data in the area give $\mathrm{P}$-wave velocities of $3.4 \mathrm{~km} / \mathrm{s}$ for sediments and $5.3-5.5 \mathrm{~km} / \mathrm{s}$ for basement [Watson and Allen 1964]. 


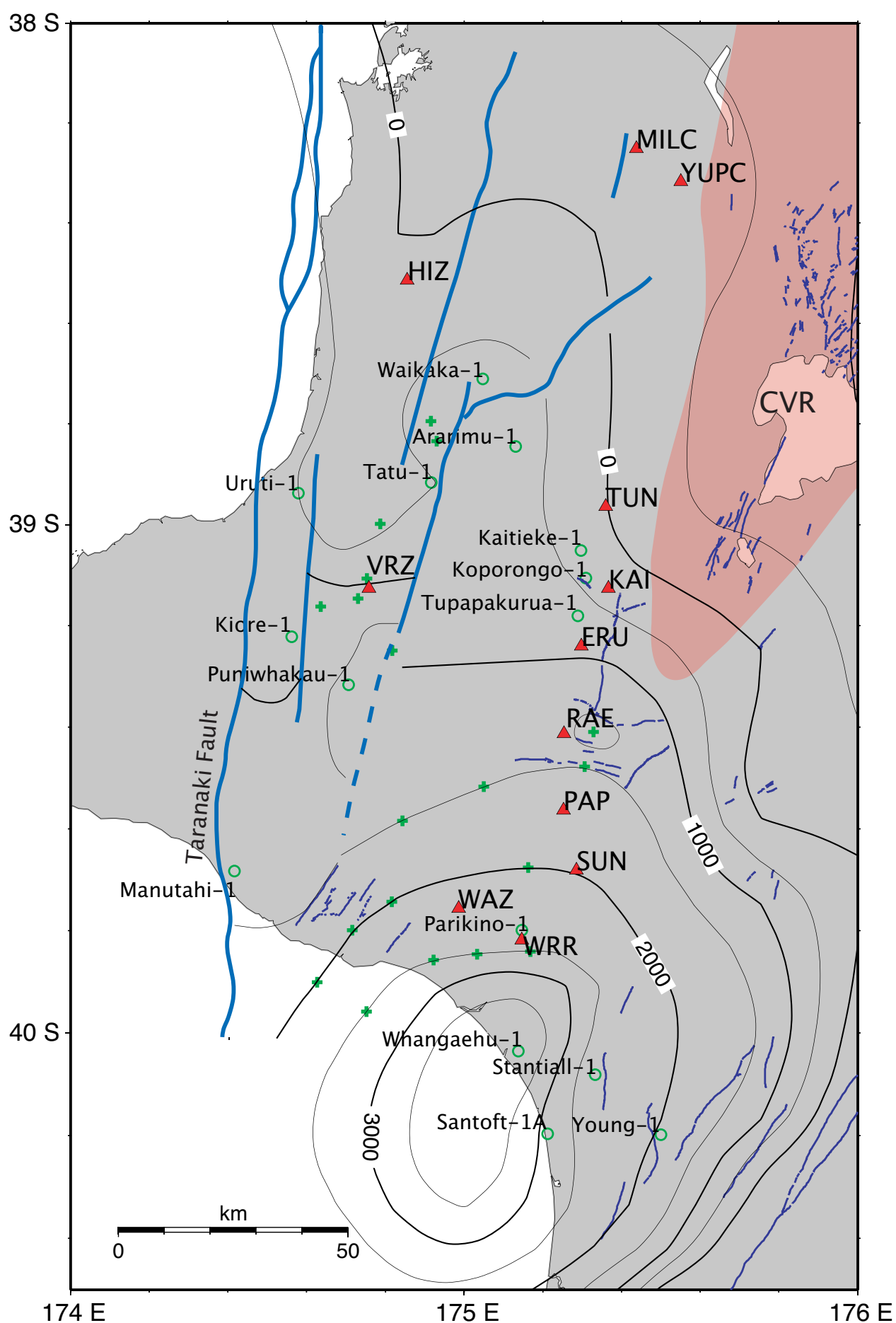

Figure 2.4: Stations location. Stations used for this study are shown as red triangles. MILC and YUPC are CNIPSE stations. HIZ, VRZ and WAZ are GEONET stations. All other stations belong to the TRAP array. The Central Volcanic Region (CVR) is shown as the shaded red region. Drill holes are shown as green circles and locations where seismic reflection data have been used to infer sediment depth are shown as green crosses. Thin blue lines indicate active faulting and thick blue lines indicate large scale faults. Basement contours are relative to mean sea level. 


\subsubsection{Earthquake event selection}

The United States Geological Survey National Earthquake Information Center global catalogue [USGS-NEIC 2005] was used to search for earthquakes with magnitudes $\geqslant$ 6.0 within an epicentral distance range of 10-110 degrees from the array. These events were then cut from the recorded data for each station using the AK135 velocity model to estimate arrival times [Kennett et al. 1995]. Waveforms were examined and only events where the $\mathrm{P}$ arrival could be seen on the vertical component (with the aid of a $0.5-2 \mathrm{~Hz}$ bandpass filter) were retained for receiver function analysis.

Receiver functions are usually carried out on teleseismic events (epicentral distances $\geqslant 30$ degrees). Regional events between 10-30 degrees were also included to increase the azimuthal coverage. Because these events will have larger angles of incidence, they may not adhere to the plane-wave assumption and their receiver functions must be interpreted with caution. Events at distances greater than 110 degrees are in the P-wave shadow zone.

Stations for the TRAP array were deployed for up to 10 months. Over this time 79 earthquakes fitted the above criteria (see Appendix B table B.1 for details), 12 regional events and 67 teleseismic events. Most of the events originate in the region of the Banda Sea and Papau to the north west of New Zealand, providing good azimuthal coverage in this quadrant (figure 2.5). Conversely there were few earthquakes recorded that originate east or south of New Zealand, giving poor azimuthal coverage in all other directions. Regional events that originate from the Tonga-Kermadec increase the azimuthal coverage to the north-east. Events to the south and north east are mostly $\geqslant$ 80 degrees away except for the Tonga-Kermadec events. Distant earthquakes will tend to have a lower signal to noise ratio (especially at higher frequencies) and will produce lower quality receiver functions. The number of events used at each station varied from 59 (at station KAI) to just 15 (at station TUN) depending on the deployment time for the station.

The GEONET stations used (HIZ, VRZ and WAZ) have all been operational since 2004. Data from 2004-2005 have been used for receiver functions. For this time period 228 events fit the criteria; 210 teleseismic events and 18 regional events (see Appendix B table B.2 for details). Again most of the events originate north west of New Zealand, however there are more events to the south and east of New Zealand than for the TRAP array, giving slightly better azimuthal coverage (figure 2.6). Regional events again mainly come from the Tonga-Kermadec region, but there are 4 events south of New Zealand in the Puysegur-Macquarie region south of New Zealand. The number of events used at each station varied from 144 (at station WAZ) to 218 (at station VRZ) depending on the deployment time for the station.

Data from the two western stations of the CNIPSE line were used; these stations are 


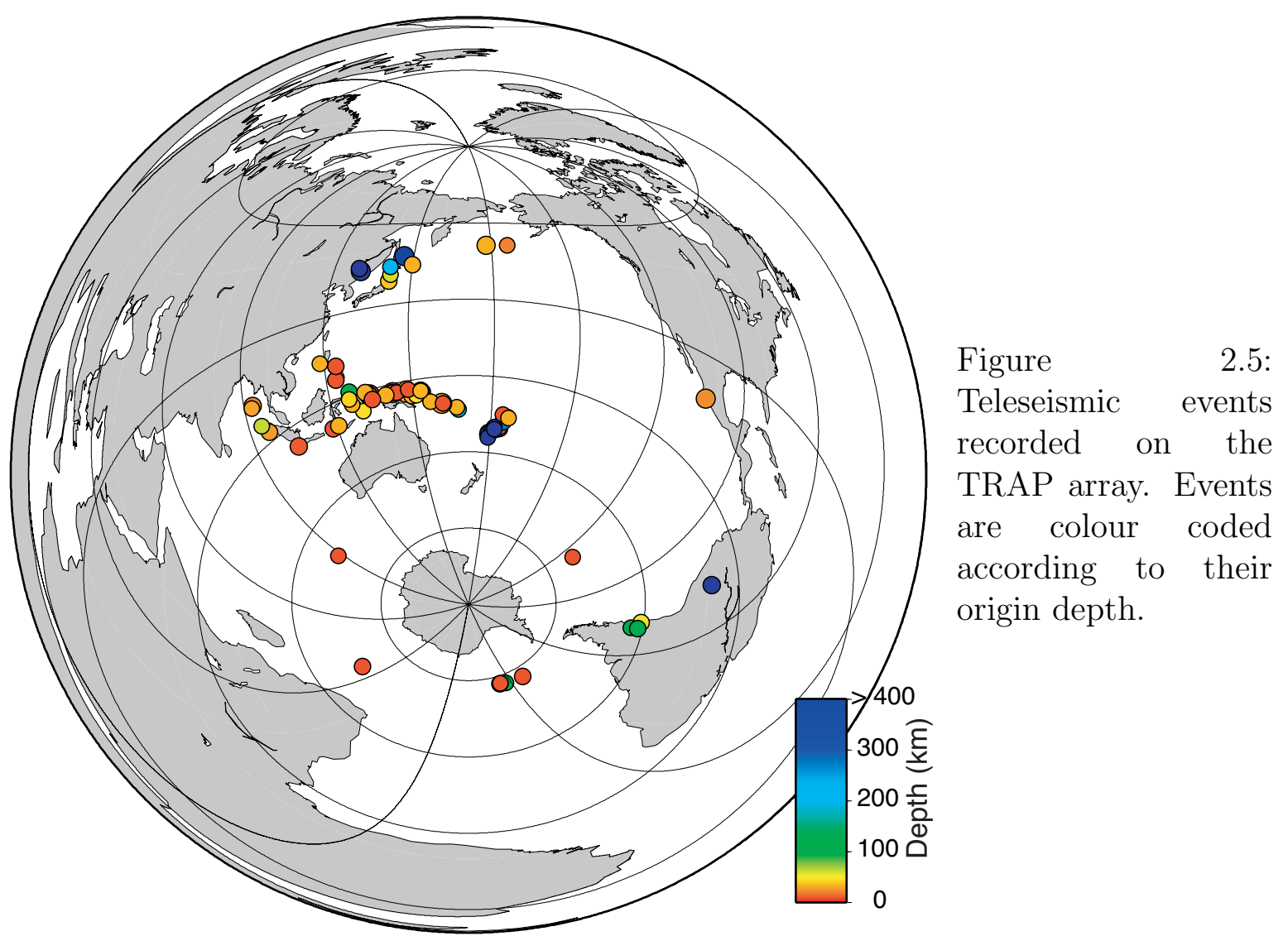

situated west of the Central Volcanic Region. Although receiver functions have been calculated for these two stations previously [Bannister et al. 2007] they have been recalculated here using the same parameters as used for the other stations. YUPC was deployed for 12 months and recorded 60 suitable events, while MILC was only deployed for 6 months and recorded only 31 suitable events (see Appendix B table B.3 for details). The spatial distribution of earthquakes used is similar to that of the TRAP stations (figure 2.7)

\subsubsection{Receiver Function Analysis}

\section{Data Preparation}

A $200 \mathrm{~s}$ window approximately centered on the first $\mathrm{P}$ arrival was cut from the unfiltered data. The mean and trend in the data were removed, ensuring that the waveform is centered at 0 amplitude. The data were then down-sampled where necessary to $50 \mathrm{~Hz}$. The horizontal components were rotated to radial and transverse orientation using the events locations from the NEIC database.

Data has also been rotated into the LQT reference frame. Due to its high frequency nature, the data could not be rotated into the LQT reference frame by maximising the energy on the L component. Low velocity sediments beneath most of the stations in this study will rotate the incoming rays so that the $\mathrm{Pp}$ phase is close to vertical and P-S 


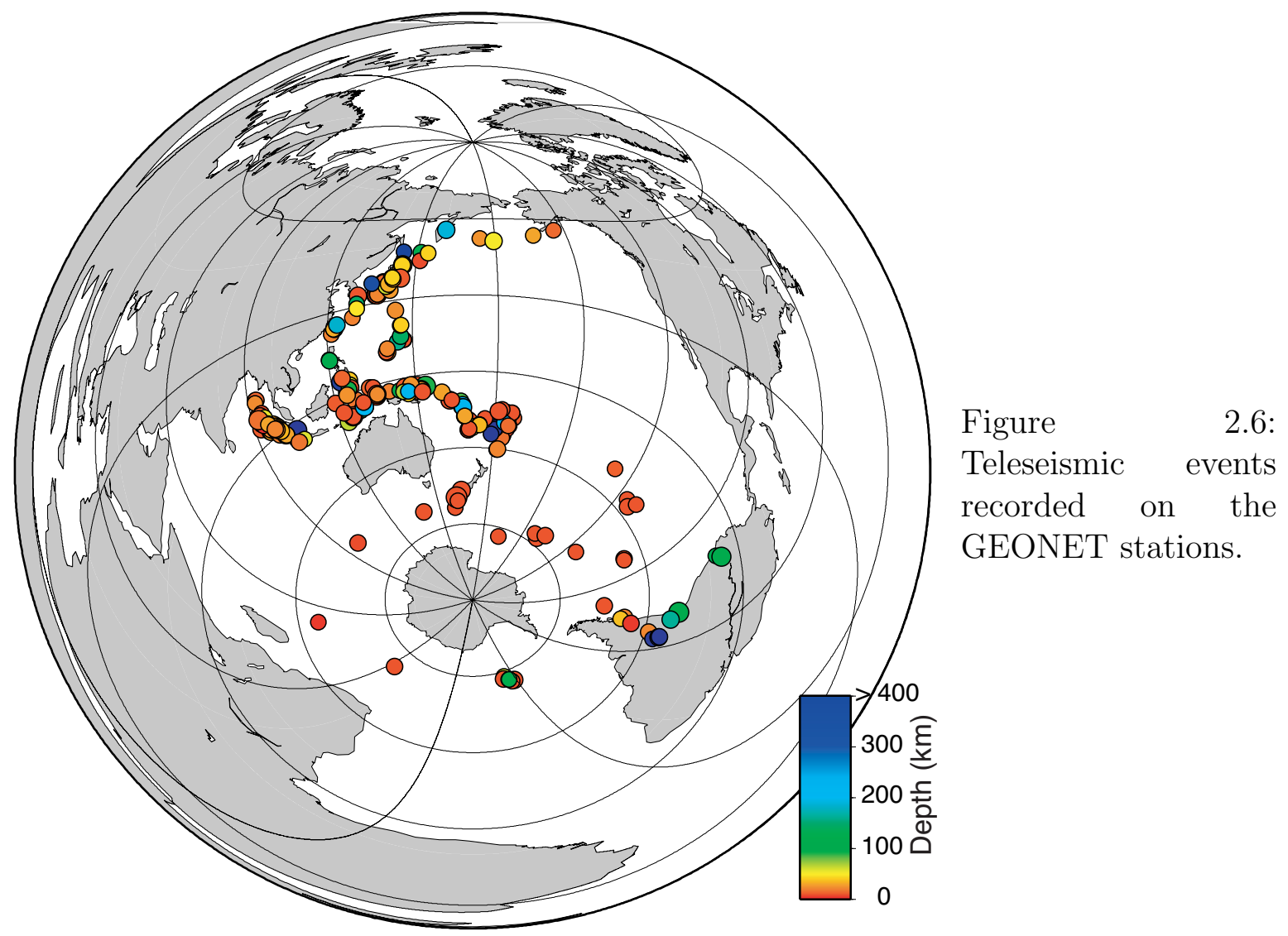

conversions are close to horizontal. Receiver functions have therefore been calculated for both the ZRT reference frame and two LQT reference frames for P-wave velocities of $3.5 \mathrm{~km} / \mathrm{s}$ and $6 \mathrm{~km} / \mathrm{s}$, corresponding to the sediment $\mathrm{P}$ wave velocity and an average crustal $\mathrm{P}$ wave velocity. Rotations for the LQT reference frame are calculated using a station-event ray parameter $(p)$, estimated from the AK135 travel time tables for the direct P arrival [Kennett et al. 1995], and Snell's law (equation 2.3.1).

$$
\frac{\sin i}{c}=p
$$

where $i$ is the angle of the incoming $\mathrm{P}$ wave for a velocity $c$.

While it is more common for broad-band instruments to be used for receiver function studies, teleseismic events were recorded on the TRAP stations with signal to noise ratios greater than 2 out to frequencies greater than $3 \mathrm{~Hz}$. Figures 2.8 and 2.9 show time domain and spectral data for a teleseismic event with an epicentral distance of $92^{\circ}$ recorded at station KAI on the vertical and radial components respectively. The highest signal amplitude is centered around $1 \mathrm{~Hz}$ ( the natural frequency of the seismometer) but signal with SNR $>2$ is present out to $3 \mathrm{~Hz}$ for both components.

Such high frequency data can be problematic for both the spectral division and time domain deconvolution methods of calculating receiver functions. The multiple-taper correlation (MTC) method of Park and Levin [2000] has been used to calculate receiver 


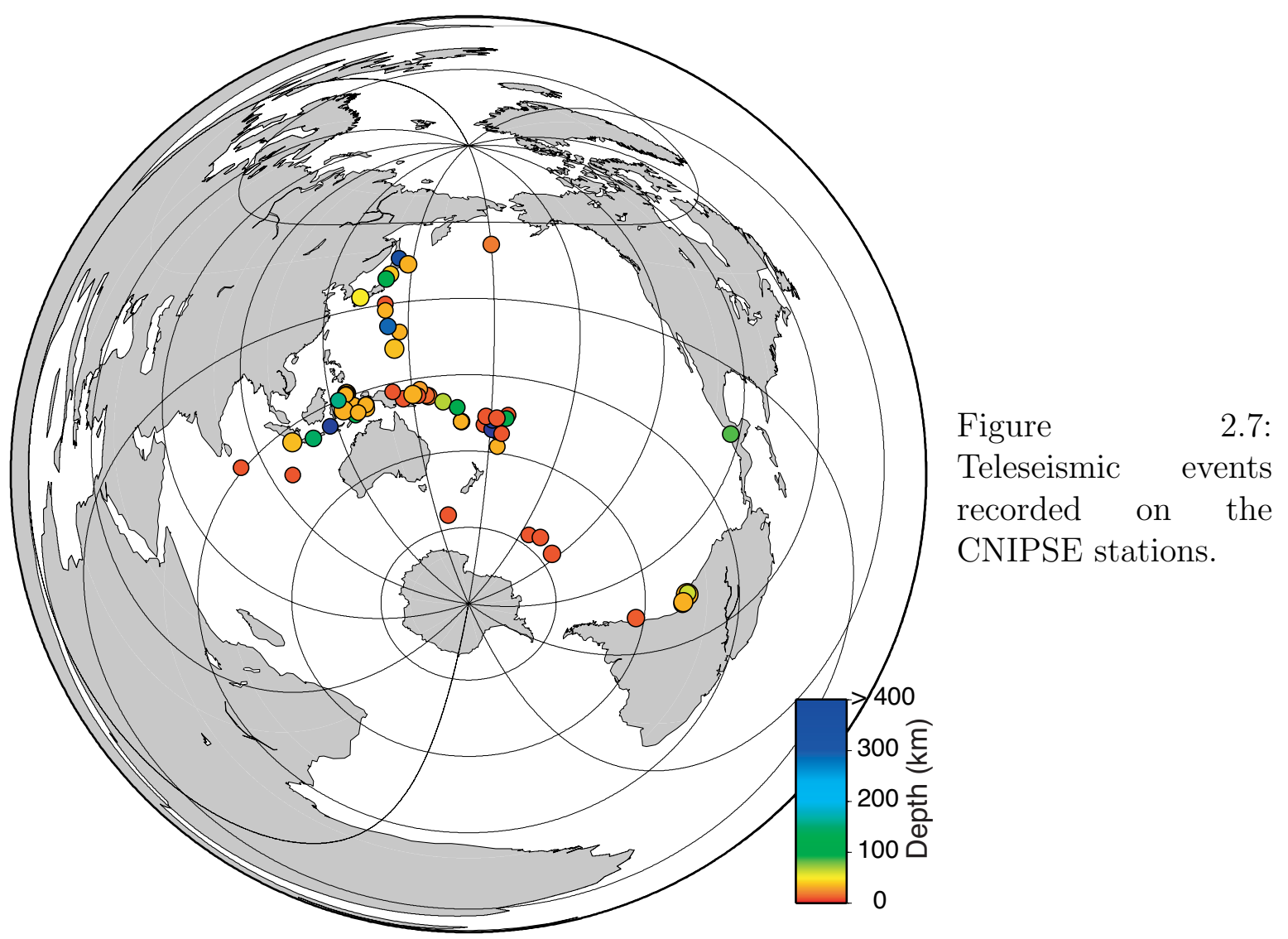

functions.

\section{Multiple-taper correlation method}

The MTC method has advantages over the two other popular receiver function calculation methods, spectral division and time domain deconvolution, especially for high frequency data. Both the spectral division and time domain methods tend to be band limited and offer no formal uncertainties with which to weight individual receiver functions for stacking. Spectral division methods require the use of a water level deconvolution to be numerically stable, which obscures low amplitude spectral components. Time domain deconvolution methods are dominated by frequencies in the signal with the highest amplitudes, often limiting the data used to frequencies $<0.5 \mathrm{~Hz}$. Neither the spectral division methods nor time domain deconvolution methods account for seismic noise. The MTC method can be used on wide frequency ranges and provides a formal uncertainty for the receiver function in the frequency domain.

Like spectral division, the MTC method is a frequency domain method of calculating receiver functions. To avoid the problem of numerical instability, cross-correlation of the horizontal and vertical component spectra is used instead of spectral division. Multiple tapers, which sample different portions of the time signal, are used to estimate the spectra. This minimises spectral leakage [Park et al. 1987] and allows low 


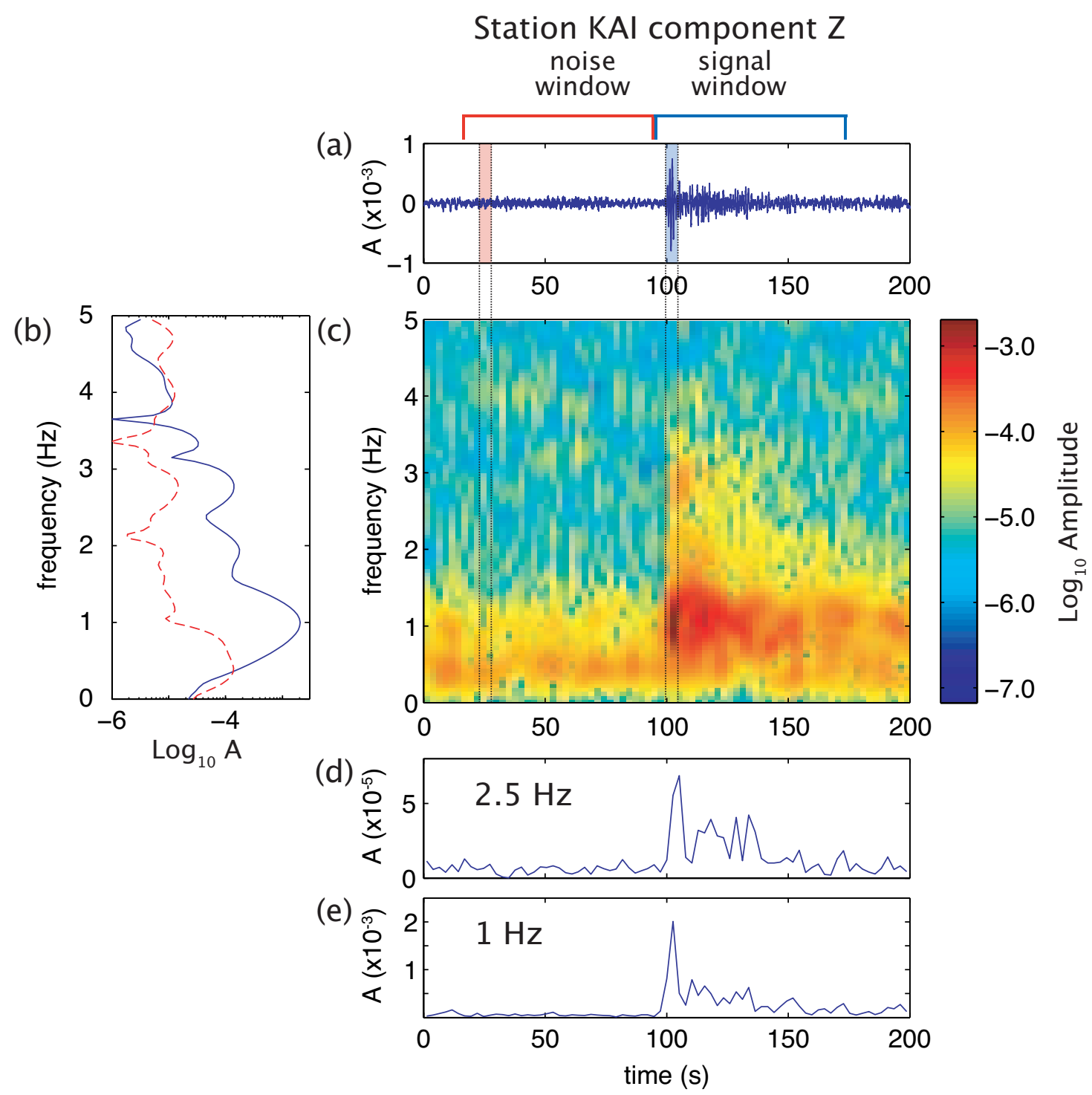

Figure 2.8: Vertical component spectrogram for an event at 2003256 22:28:29 recorded at station KAI. All amplitudes (A) are measured in volts. This event occurred at an epicentral distance of $92^{\circ}$ from the station. (a) shows the signal in the time domain. The $\mathrm{P}$ wave arrives at $\sim 100 \mathrm{~s}$. Noise and signal windows used in receiver function calculations are indicated above the plot. The pink and blue shaded areas indicate $5 \mathrm{~s}$ time windows used to compute the spectra shown in (b). The pink window is a noise window corresponding to the red dashed line in (b). The blue window encompasses the first few seconds of the P signal and corresponds to the solid blue line in (b). (c) is the spectrogram for the event and shows amplitudes plotted against both time and frequency, the dotted lines indicate the time windows used in (b). (d) and (e) are plots of amplitude vs. time for $2.5 \mathrm{~Hz}$ and $1 \mathrm{~Hz}$ frequencies respectively. 


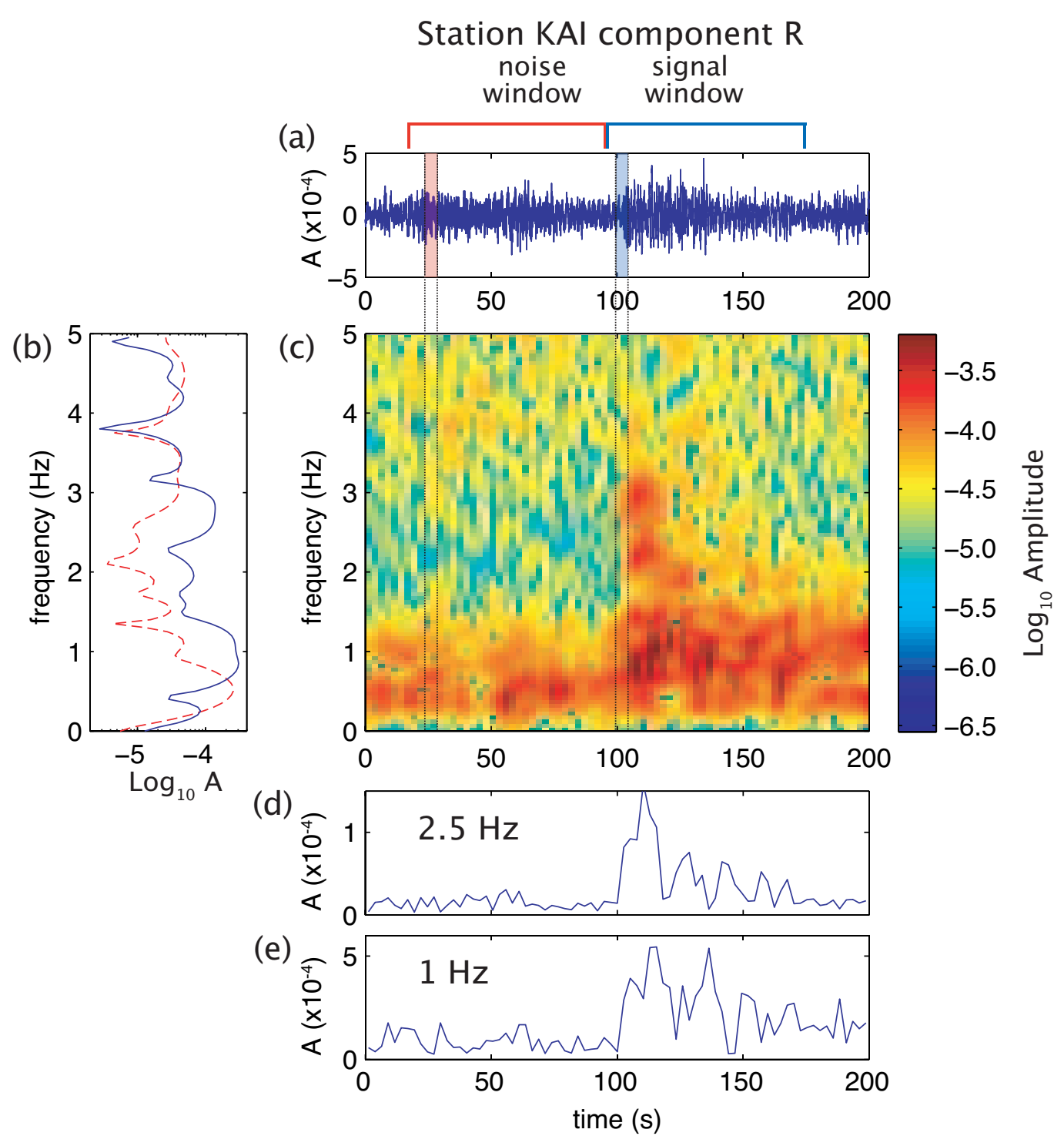

Figure 2.9: Radial component spectrogram for the same event shown in figure 2.8 recorded at station KAI. All amplitudes (A) are measured in volts. This event occurred at an epicentral distance of $92^{\circ}$ from the station. (a) shows the signal in the time domain. The $\mathrm{P}$ wave arrives at $\sim 100 \mathrm{~s}$. Noise and signal windows used in receiver function calculations are indicated above the plot. The pink and blue shaded areas indicate $5 \mathrm{~s}$ time windows used to compute the spectra shown in (b). The pink window is a noise window corresponding to the red dashed line in (b). The blue window encompasses the first few seconds of the $\mathrm{P}$ signal and corresponds to the solid blue line in (b). (c) is the spectrogram for the event and shows amplitudes plotted against both time and frequency, the dotted lines indicate the time windows used in (b). (d) and (e) are plots of amplitude vs. time for $2.5 \mathrm{~Hz}$ and $1 \mathrm{~Hz}$ frequencies respectively. 
amplitude portions of the spectrum to be used in calculating receiver functions, while minimising the amount of data discarded by tapering. This has the effect of increasing the bandwidth which can be used. The MTC method also accounts for noise by using the pre-event noise spectrum for frequency dependent damping. By calculating the coherence of the vertical and horizontal components, a distinction between coherent and incoherent signal for a single event at a single station can be made and formal uncertainties in the frequency domain can be estimated. Formal uncertainties allow weighted stacking of many events to improve the receiver function image.

To calculate the receiver function using the MTC method, the multiple-taper spectrum estimates must first be calculated. For a finite time series $u(n \tau)$, where $n$ is the sample number and $\tau$ is the sample interval, the spectrum estimate at each frequency $f$ is calculated for a series of prolate eigentapers [Park et al. 1987] using equation 2.3.2.

$$
Y_{\gamma}^{k}(f)=\sum_{n=0}^{N-1} u_{n}^{\gamma} w_{n}^{(k)} e^{i 2 \pi f n \tau}
$$

where $\gamma$ is the component (Z,R,T or L,Q,T), N is the number of samples and $w_{n}^{(k)}$ is the $k$ th taper for a time-bandwidth product $p$ [Park et al. 1987, Vernon et al. 1991]. For this study three tapers are used $(K=3)$ and $p$ is set to a value of 2.5 (figure 2.10). These values are appropriate for receiver functions with Ps conversions in the first 100 $\mathrm{km}$ of the crust and upper mantle [Park and Levin 2000].

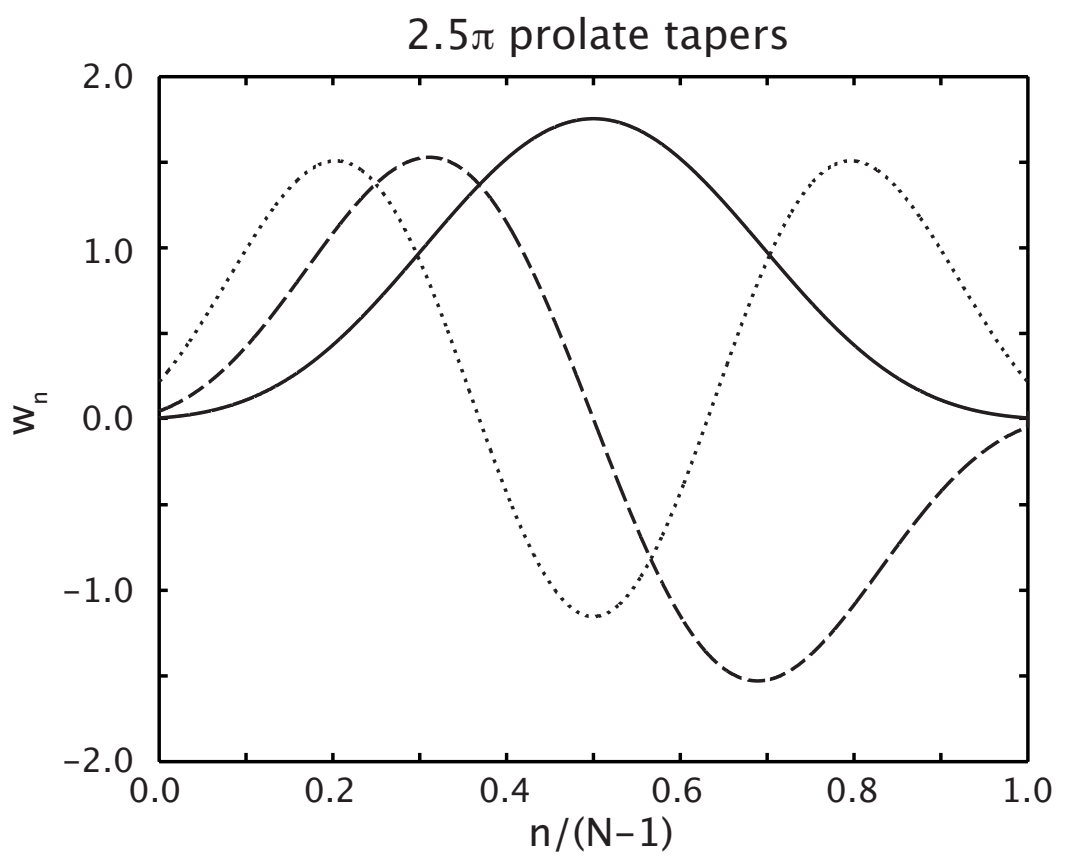

Figure 2.10: Prolate eigen tapers used. The solid line is the taper for $\mathrm{k}=1$, the dashed line is for $\mathrm{k}=2$ and the dotted line is for $\mathrm{k}=3$.

The radial and transverse receiver functions, $H_{R}(f)$ and $H_{T}(f)$, can then be calculated 
in the frequency domain using damped spectral correlation estimators:

$$
\begin{aligned}
& H_{R}(f)=\frac{\sum_{k=0}^{K-1}\left(Y_{Z}^{(k)}(f)\right) * Y_{R}^{(k)}(f)}{\left(\left(\sum_{k=0}^{K-1}\left(Y_{Z}^{(k)}(f)\right) * Y_{Z}^{(k)}(f)\right)+S_{0}(f)\right)} \\
& H_{T}(f)=\frac{\sum_{k=0}^{K-1}\left(Y_{Z}^{(k)}(f)\right) * Y_{T}^{(k)}(f)}{\left(\left(\sum_{k=0}^{K-1}\left(Y_{Z}^{(k)}(f)\right) * Y_{Z}^{(k)}(f)\right)+S_{0}(f)\right)}
\end{aligned}
$$

where the frequency dependent damping, $S_{0}(f)$, is a spectrum estimate of the pre-event noise on the vertical component calculated using equation 2.3.2. Converted phases can be retrieved for time delays up to $(N-1) \tau / 2 p$ using the MTC method.

How well the horizontal and vertical components correlate can be quantified by calculating the coherence:

$$
\begin{gathered}
C_{R}(f)=\frac{\sum_{k=0}^{K-1}\left(Y_{Z}^{(k)}(f)\right) * Y_{R}^{(k)}(f)}{\left(\left(\sum_{k=0}^{K-1}\left(Y_{R}^{(k)(f)} * Y_{R}^{(k)}(f)\right)\left(\sum_{k=0}^{K-1}\left(Y_{Z}^{(k)}(f)\right) * Y_{Z}^{(k)}(f)\right)\right)^{1 / 2}\right.} \\
C_{T}(f)=\frac{\sum_{k=0}^{K-1}\left(Y_{Z}^{(k)}(f)\right) * Y_{T}^{(k)}(f)}{\left(\left(\sum_{k=0}^{K-1}\left(Y_{T}^{(k)}(f) * Y_{T}^{(k)}(f)\right)\left(\sum_{k=0}^{K-1}\left(Y_{Z}^{(k)}(f)\right) * Y_{Z}^{(k)}(f)\right)\right)^{1 / 2}\right.}
\end{gathered}
$$

where $C_{R}(f)$ is the coherence for the radial component and $C_{T}(f)$ is the coherence for the transverse component. The closer the coherence value is to 1 the better correlated the components are. The coherence can be used to calculate formal uncertainties for the receiver functions in the frequency domain:

$$
\begin{aligned}
& \operatorname{var}\left(H_{R}(f)\right)=\left(\frac{1-\left(C_{R}(f)\right)^{2}}{(K-1)\left(C_{R}(f)\right)^{2}}\right)\left|H_{R}(f)\right|^{2} \\
& \operatorname{var}\left(H_{T}(f)\right)=\left(\frac{1-\left(C_{T}(f)\right)^{2}}{(K-1)\left(C_{T}(f)\right)^{2}}\right)\left|H_{T}(f)\right|^{2}
\end{aligned}
$$

Incoherent signal can be attributed to background noise and signal generated noise such as body-surface wave conversions.

MTC receiver functions are calculated using both 60 second and 80 second time win- 
dows, which correspond to maximum delay times of 12 seconds and 16 seconds, respectively. The shorter time window allows better resolution at short delay times and the longer time windows allows the inclusion of conversions with longer delay times. Pre-event noise windows and signal windows are of equal length. Noise windows end $\sim 5 \mathrm{~s}$ before the predicted $\mathrm{P}$ wave arrival and signal windows begin immediately after the noise window (figure 2.8 and 2.9 show $80 \mathrm{~s}$ windows).

Frequency domain receiver functions are low pass filtered using a cosine-squared filter, $\cos ^{2}\left(\frac{\pi f}{2 f_{c}}\right)$, to prevent ringing. Receiver functions are calculated using four different corner frequencies $\left(f_{c} 1.0-2.5 \mathrm{~Hz}\right)$. Higher frequency receiver functions provide more detail for shallow structure while lower frequency receiver functions give a better representation of deeper structure. Frequency domain receiver functions for individual events are sorted into stacking bins for both epicentral distance and back azimuth. Overlapping bins of $10^{\circ}$ width are used so that each event contributes to two adjacent bins. The inverse-variances of the individual receiver functions are used as weights for stacking so that frequencies with a low coherence between the vertical and horizontal components will influence the stacked receiver function less than those that are strongly coherent.

Time domain receiver functions for the stacked spectra are calculated using an inverse Fourier transform and are normalised to preserve amplitudes using the normalisation factor $2 f_{N} / f_{c}$ where $f_{N}$ is the Nyquist frequency. There are no formal uncertainties for receiver functions in the time domain.

\subsection{Receiver function observations}

Receiver functions were calculated for four different low-pass filters. The following section illustrates the results for a $1.5 \mathrm{~Hz}$ cut off frequency and $80 \mathrm{~s}$ time windows. A $1.5 \mathrm{~Hz}$ cut off frequency is approximately equivalent to a Gaussian filter with an $a$ value of 2.5 , which results in a pulse width of $\sim 1 \mathrm{~s}$.

\subsubsection{TRAP stations}

\section{TUN}

Receiver functions for station TUN are shown in figure 2.11. TUN is the northern most station in the TRAP array and had the shortest deployment time. This station only recorded 15 events that could be used for receiver function analysis, thus limiting the back-azimuth range and the number of events in each stacking bin.

Radial receiver functions for this station do not have a positive peak at 0 time delay 
where the Pp arrival should be. Instead they show a strong positive peak with a small time delay $(\sim 0.3 \mathrm{~s})$ followed by a negative peak at $1.4 \mathrm{~s}$. There is another positive peak at $3.2 \mathrm{~s}$, which is strongest in the $315-335^{\circ}$ back-azimuth range. This peak is also evident in between $30^{\circ}$ and $50^{\circ}$ in the epicentral distance stacks.

The transverse receiver functions show a small negative peak over a back-azimuth range of $280^{\circ}-315^{\circ}$ at the same delay time as the first positive peak on the radial receiver functions. This negative peak is followed by a positive peak at a delay time of 0.9 s. The only peak that is coherent across both back-azimuth and epicentral distance ranges for the transverse receiver functions is a negative peak at $5 \mathrm{~s}$. Rotation into the LQT reference frame does not provide any additional information for this station.
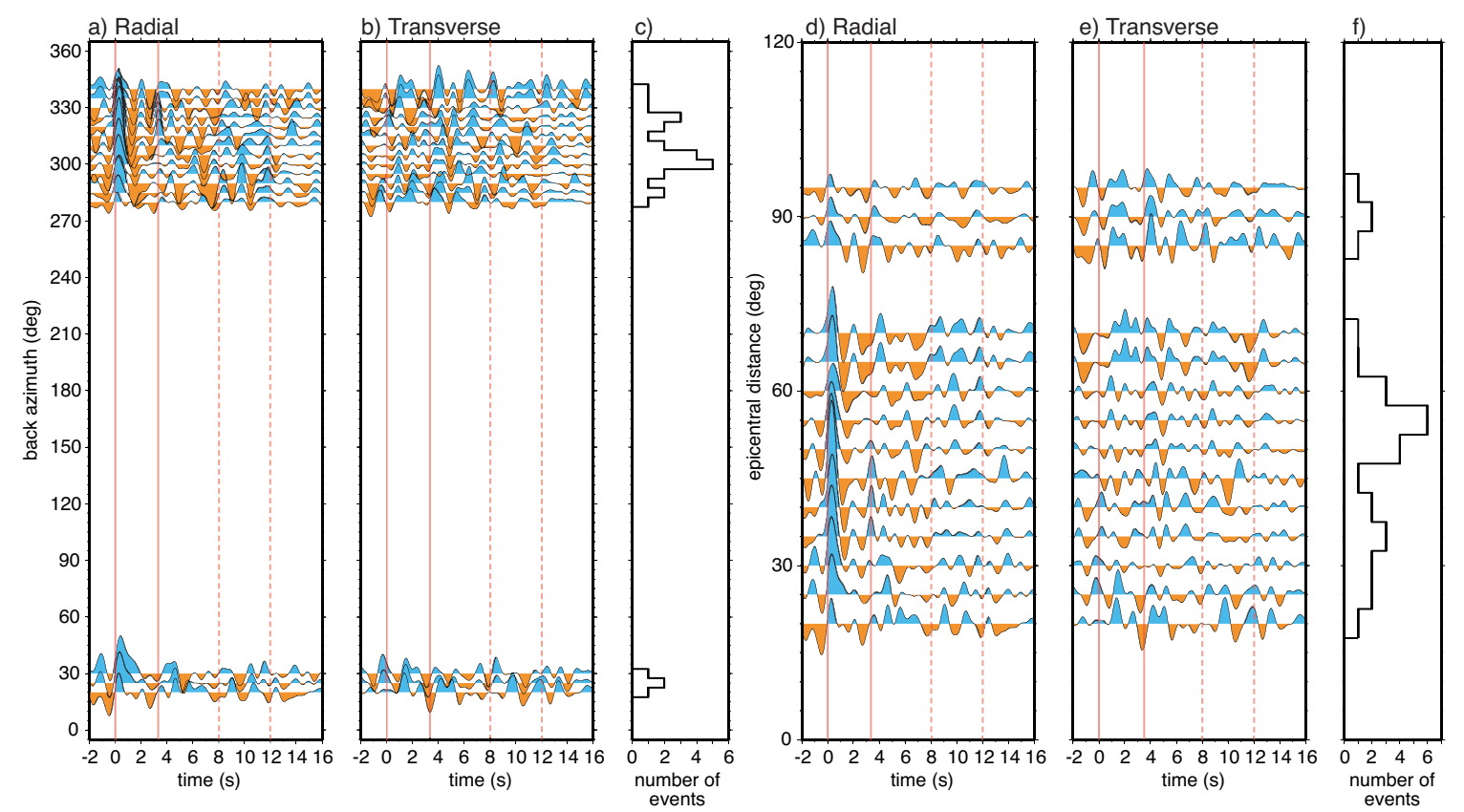

Figure 2.11: TUN receiver functions. Blue indicates a positive phase and red indicates a negative phase. (a) and (b) show radial (R) and transverse $(\mathrm{T})$ receiver functions stacked in back-azimuth bins. (d) and (e) show $\mathrm{R}$ and $\mathrm{T}$ receiver functions stacked in epicentral distance bins. (c) and (f) show the number of events in each stacking bin for back-azimuth and epicentral distance stacks respectively. Solid red lines indicate the $0 \mathrm{~s}$ and $3.2 \mathrm{~s}$ delay times and dashed red lines are at $8 \mathrm{~s}$ and $12 \mathrm{~s}$.

\section{KAI}

Station KAI recorded 59 events that could be used in receiver function analysis. This station had the best azimuthal coverage of all the TRAP stations. As with station TUN, radial receiver functions for KAI do not have a peak at a 0 second delay time (figure 2.12). The first positive peak on the radial receiver functions is slightly offset from zero $(0.1 \mathrm{~s})$. This peak is followed by a negative peak that shifts from $2.2 \mathrm{~s}$ at an epicentral distance of $15^{\circ}$ to $1.5 \mathrm{~s}$ at epicentral distances $\geqslant 60^{\circ}$. A positive peak is observed on the radial receiver functions at 3.3 seconds. This peak is strongest for back-azimuths between $300^{\circ}$ and $345^{\circ}$ and can be traced over the entire epicentral 
distance range. A positive peak can be observed at $\sim 5 \mathrm{~s}$ for back-azimuths between $50^{\circ}$ and $80^{\circ}$.

Two additional positive peaks can be seen on the radial receiver functions, one between 7 and $8 \mathrm{~s}$ and another at between 11 and $12 \mathrm{~s}$. The first of these two peaks has decreasing delay times with increasing epicentral distance consistent with it being a Ps conversion from within the mantle. The delay time for the 11-12 s peak increases with increasing epicentral distance. This is consistent with the move out expected for a multiple. There is also a negative peak observed at $\sim 5.8 \mathrm{~s}$.

There is a significant amount of energy on the transverse receiver functions. A negative peak at $0.3 \mathrm{~s}$ is observed in the back-azimuth range between $275^{\circ}$ and $310^{\circ}$. The $3.3 \mathrm{~s}$ positive peak on the radial receiver functions can also be seen on the transverse receiver functions, however it is preceded by a sharp negative pulse. The back-azimuth binned receiver functions also have weak peaks at 8 and $11 \mathrm{~s}$ delay times on the transverse components, however the polarity of the $8 \mathrm{~s}$ peak is opposite to that observed in the radial receiver functions.

The coherency of the $3.3 \mathrm{~s}$ peak is improved by rotating the data into the LQT reference frame. Figure 2.13 shows the $\mathrm{R}$ and $\mathrm{Q}$ receiver function results for epicentral distance stacks. On these plots the moveout for the 7-8 s and 11-12 s peaks can be seen more clearly. The positive phase at $\sim 5 \mathrm{~s}$ can also be traced over a larger epicantral distance range. The delay time for this phase increases with increasing epicentral distance (4.5 $\mathrm{s}$ at $20^{\circ}$ to $5 \mathrm{~s}$ at $80^{\circ}$ ) indicating that it is a multiple. The negative phases between 1.5 and $2.2 \mathrm{~s}$ and at $\sim 5.8 \mathrm{~s}$ reduce in amplitude in the LQT reference frame, suggesting that these may have a $\mathrm{P}$ multiple component.

\section{ERU}

Station ERU recorded 52 teleseismic events. The radial receiver functions are characterised by a band of positive energy out to $1.6 \mathrm{~s}$ followed by a band of negative energy out to $3.2 \mathrm{~s}$ (figure 2.14). Both of these bands have a double peak. Positive peaks are observed at $3.4 \mathrm{~s}$ and at $\sim 8 \mathrm{~s}$ and a negative peak is observed at $5.3 \mathrm{~s}$. The $8 \mathrm{~s}$ peak is only a weak feature of the epicentral distance stacks.

The transverse receiver functions have a positive peak at $1.9 \mathrm{~s}$ which matches the timing of the first peak in the negative band on the radial receiver functions. There is a weak positive peak at $3.4 \mathrm{~s}$ followed by a stronger positive at $4.6 \mathrm{~s}$.

The coherency of the $3.4 \mathrm{~s}$ peak is improved by rotating the data into the LQT reference frame. Figure 2.15 shows the Q component results for epicentral distance stacks. The coherency of the $\sim 8 \mathrm{~s}$ phase out to $\sim 60^{\circ}$ epicentral distance is also enhanced in the LQT plots. The second peak in the 1.6-3.2 s negative band reduces in amplitude with rotation into LQT, indicative of this being a $\mathrm{P}$ multiple from a near surface boundary. 

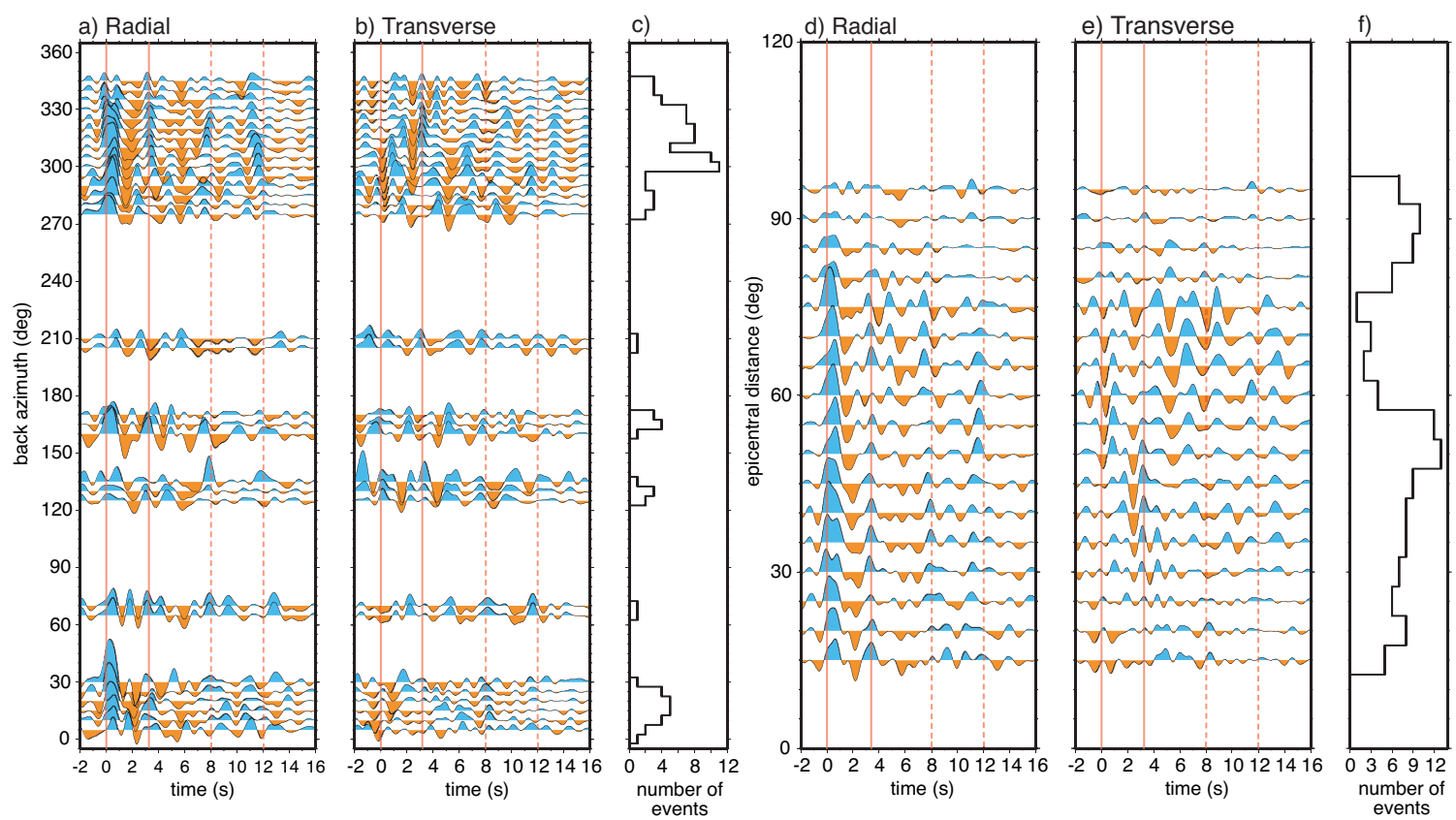

Figure 2.12: KAI receiver functions. Blue indicates a positive phase and red indicates a negative phase. (a) and (b) show $\mathrm{R}$ and $\mathrm{T}$ receiver functions stacked in back-azimuth bins. (d) and (e) show $\mathrm{R}$ and $\mathrm{T}$ receiver functions stacked in epicentral distance bins. (c) and (f) show the number of events in each stacking bin for back-azimuth and epicentral distance stacks respectively. Solid red lines indicate the $0 \mathrm{~s}$ and $3.3 \mathrm{~s}$ delay times. Dashed red lines are at $8 \mathrm{~s}$ and $12 \mathrm{~s}$.
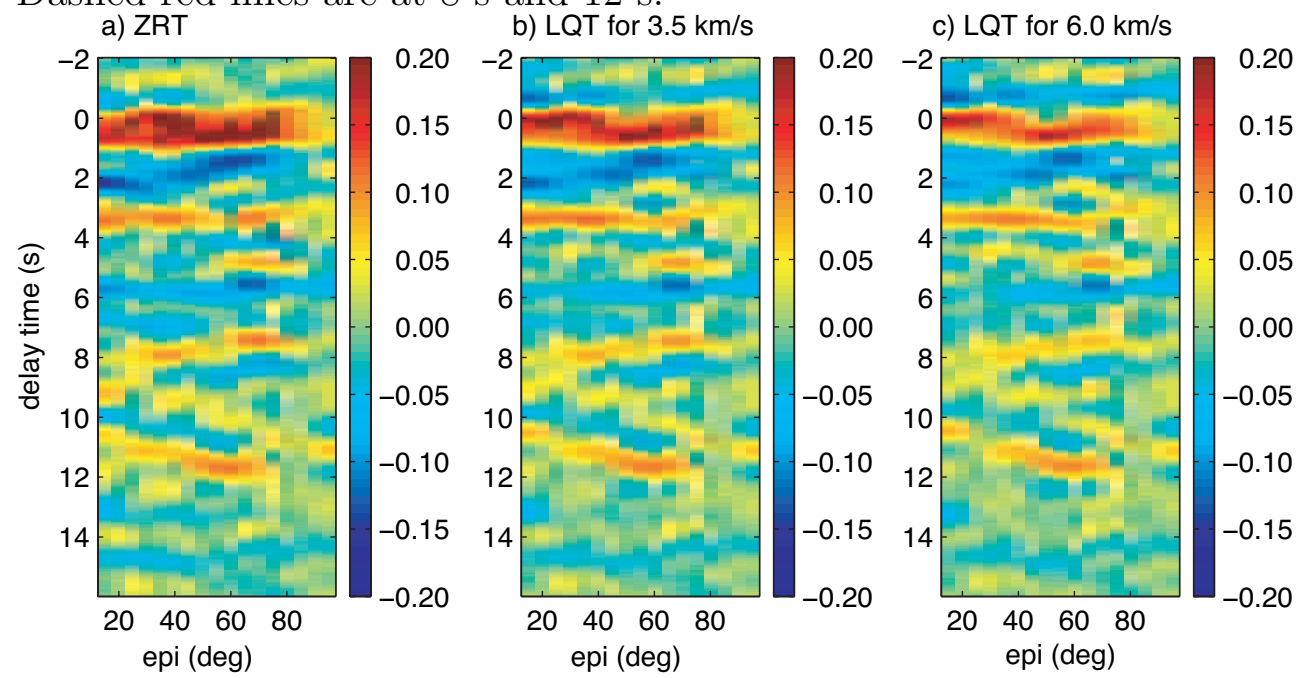

Figure 2.13: Epicentral distance stacked receiver functions in the LQT reference frame for station KAI. Here red indicates a positive phases. (a) shows the same stack as figure $2.12 \mathrm{~d}$ ) for the ZRT reference frame. (b) and (c) show Q stacks for receiver functions calculated using the LQT reference frame with (b) rotating for a top layer velocity of $3.5 \mathrm{~km} / \mathrm{s}$ and (c) rotating for a top layer velocity of $6.0 \mathrm{~km} / \mathrm{s}$. For both LQT stacks the positive arrivals at $3.3 \mathrm{~s}, 5 \mathrm{~s}, 8 \mathrm{~s}$ and $12 \mathrm{~s}$ appear more coherent across the epicentral distance range. 

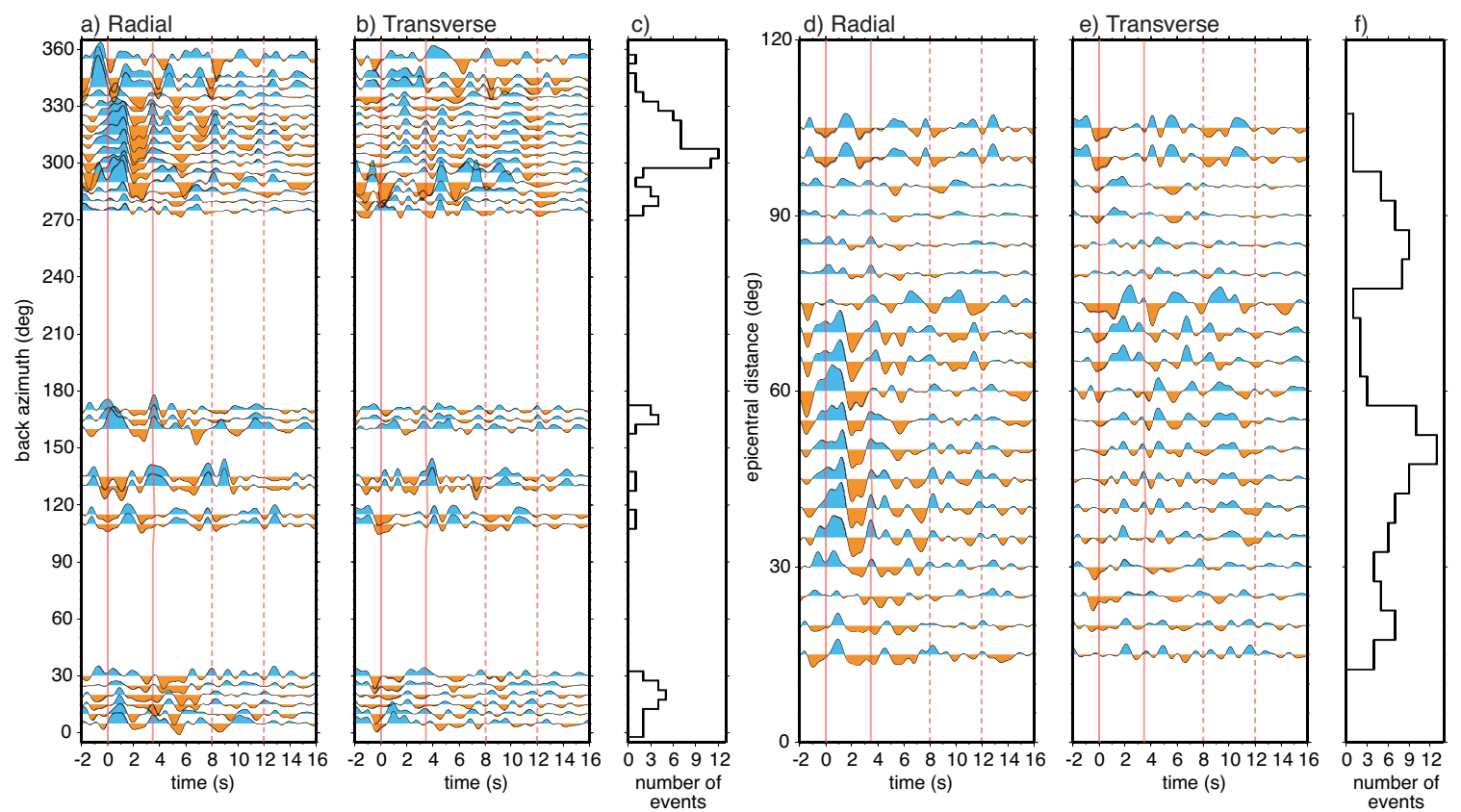

Figure 2.14: ERU receiver functions. Blue indicates a positive phase and red indicates a negative phase. (a) and (b) show $\mathrm{R}$ and $\mathrm{T}$ receiver functions stacked in back-azimuth bins. (d) and (e) show $\mathrm{R}$ and $\mathrm{T}$ receiver functions stacked in epicentral distance bins. (c) and (f) show the number of events in each stacking bin for back-azimuth and epicentral distance stacks respectively. Solid red lines indicate the 0 and 3.4 s delay times. The dashed red lines are at 8 and 12 seconds.
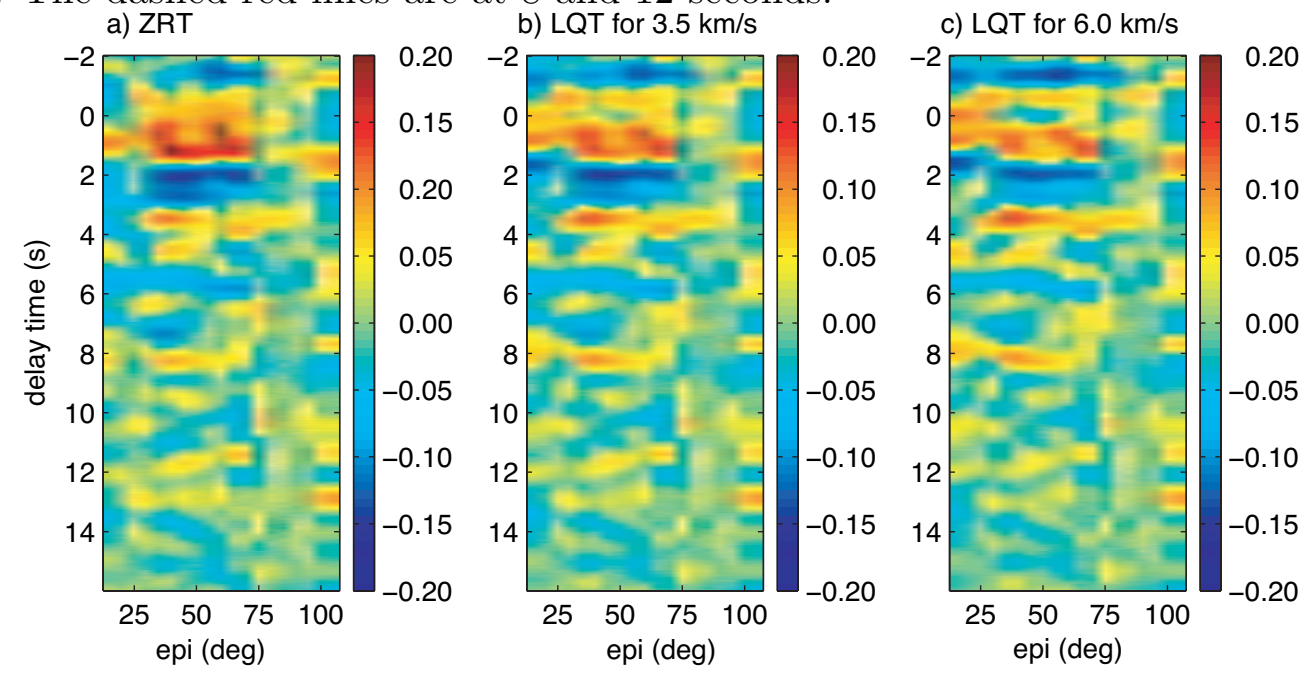

Figure 2.15: Epicentral distance stacked receiver functions in the LQT reference frame for station ERU. Here red indicates a positive phases. (a) shows the same stack as figure $2.14 \mathrm{~d}$ ) for the ZRT reference frame. (b) and (c) show Q stacks for receiver functions calculated using the LQT reference frame with (b) rotating for a top layer velocity of $3.5 \mathrm{~km} / \mathrm{s}$ and (c) rotating for a top layer velocity of $6.0 \mathrm{~km} / \mathrm{s}$. For both LQT stacks the positive arrival at $3.4 \mathrm{~s}$ is more coherent across the epicentral distance range. 


\section{RAE}

The RAE station only recorded 28 events that could be used for receiver function analysis, since one of the horizontal components was malfunctioning for 5 months. Receiver functions show a similar pattern to those of station ERU. Radial receiver functions have a double peaked region of positive energy out to delay times of $2.1 \mathrm{~s}$ followed by a similar double peaked negative out to $4 \mathrm{~s}$ (figure 2.16).

A positive peak is observed at $4.3 \mathrm{~s}$. Receiver functions binned according to back azimuth also show positive peaks at $\sim 8 \mathrm{~s}$ and $\sim 12 \mathrm{~s}$. These arrivals are less obvious in receiver functions binned according to epicentral distance. Radial receiver functions also have a negative peak between 6 and $7 \mathrm{~s}$. This peak is largest for smaller epicentral distances and has a delay time that increases with epicentral distance, indicating that this is a multiple.

The transverse receiver functions have a positive peak at 8 s delay time for backazimuth bins between $310^{\circ}$ and $330^{\circ}$. At this same delay time the limited events from the back-azimuth range between $60^{\circ}$ and $290^{\circ}$ have a negative peak.

The coherency of the $4.3 \mathrm{~s}$ peak is improved by rotating the data into the LQT reference frame. Figure 2.17 shows the R and Q component results for epicentral distance stacks.

\section{PAP}

Station PAP recorded 45 teleseismic events. Radial receiver functions have a broad double peaked region of positive energy out to delay times of 2. s (figure 2.18), similar to both stations ERU and RAE. It is followed by a broad region of negative peaks out to $\sim 6 \mathrm{~s}$. Unlike the stations to the north, a positive peak in the $3-4 \mathrm{~s}$ delay time range is not coherent in the back-azimuth or the epicentral distance binned receiver functions. A positive peak bounded by negative peaks either side is observed at $6.3 \mathrm{~s}$ on the back-azimuth stacks. There are also weak positive peaks at $\sim 8 \mathrm{~s}$ and $\sim 12 \mathrm{~s}$.

On the transverse receiver functions a positive peak is observed at $0 \mathrm{~s}$ which is followed by a negative peak at $0.6 \mathrm{~s}$. Negative peaks that approximately match the timing for the $6.3 \mathrm{~s}$ and $12 \mathrm{~s}$ peaks on the radial component are observed over a back-azimuth ranges of $295^{\circ}-330^{\circ}$ and $310^{\circ}-345^{\circ}$ respectively. Rotation into LQT has little effect on receiver functions for this station.

\section{SUN and WRR}

Stations SUN and WRR are at the southern end of the TRAP line and recorded 55 and 35 teleseismic events respectively. Peaks on the radial components are difficult to trace over back-azimuth or epicentral distance (figure 2.20 and 2.21). Instead a region of dominantly positive energy out to $\sim 3 \mathrm{~s}$ followed by a band of dominantly negative energy out to $\sim 6 \mathrm{~s}$ is observed. Three peaks that can be traced over adjacent stacking bins for station SUN are a negative arrival at $5.6 \mathrm{~s}$ and two positives at $\sim 8 \mathrm{~s}$ and $\sim 13$ 

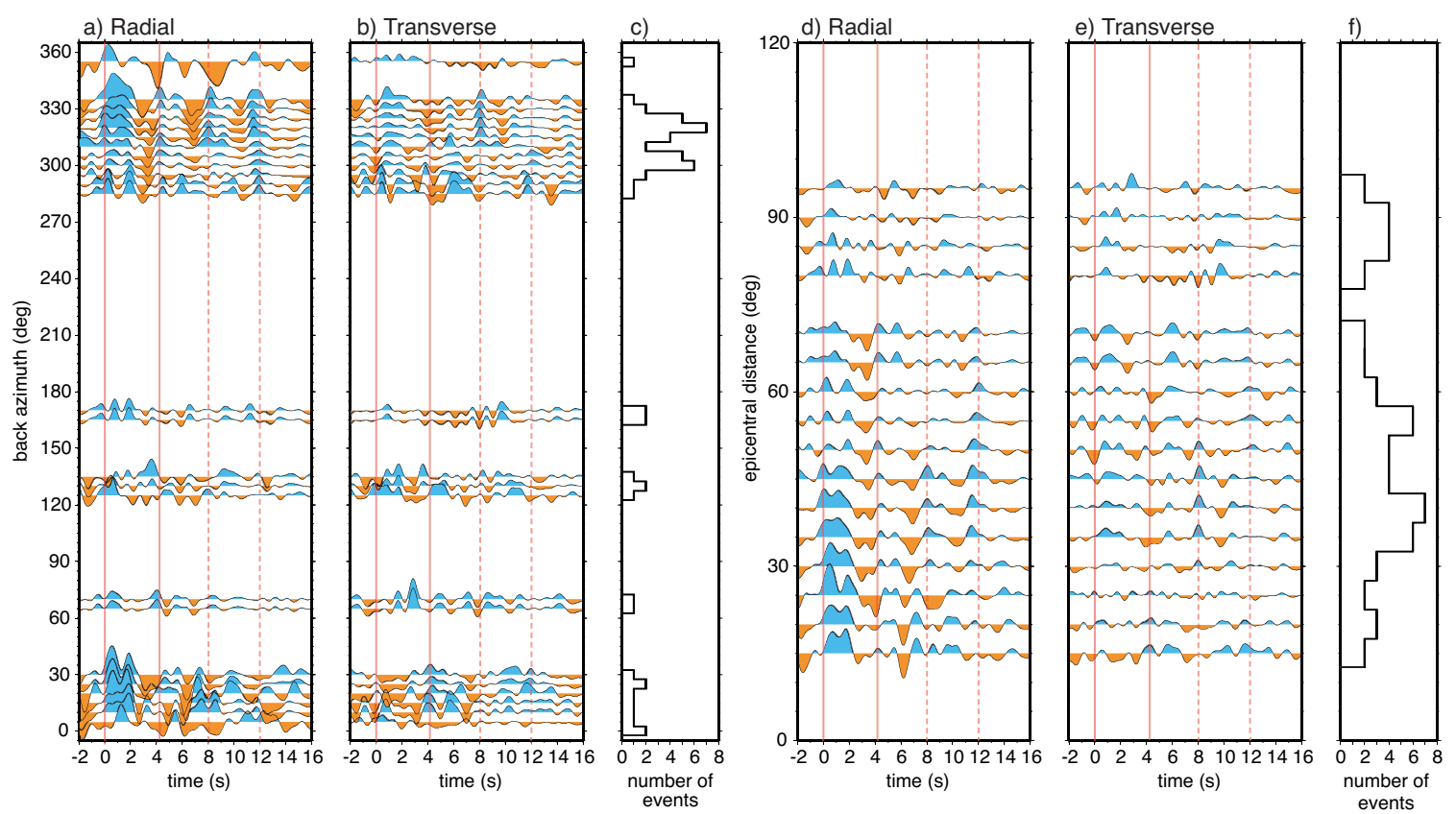

Figure 2.16: RAE receiver functions. Blue indicates a positive phase and red indicates a negative phase. (a) and (b) show $\mathrm{R}$ and $\mathrm{T}$ receiver functions stacked in back-azimuth bins. (d) and (e) show $\mathrm{R}$ and $\mathrm{T}$ receiver functions stacked in epicentral distance bins. (c) and (f) show the number of events in each stacking bin for back-azimuth and epicentral distance stacks respectively. The solid red lines indicate the 0 and $4.3 \mathrm{~s}$ delay times. The dashed red lines are at $8 \mathrm{~s}$ and $12 \mathrm{~s}$.
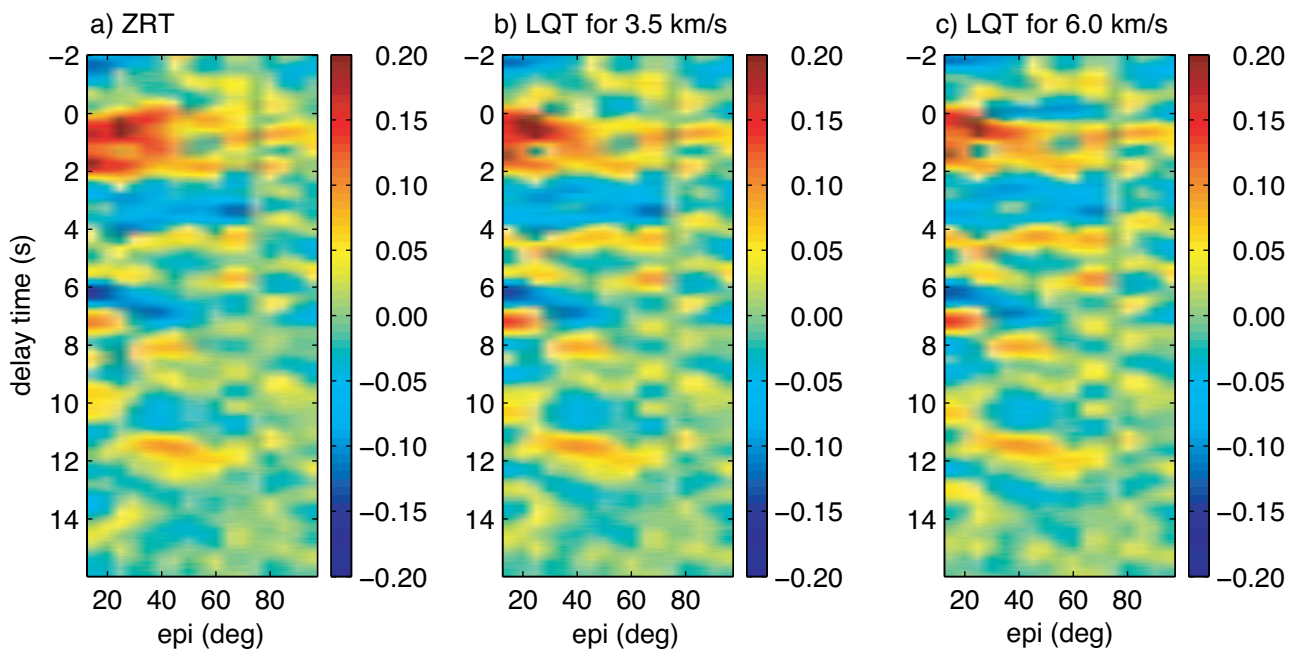

Figure 2.17: Epicentral distance stacked receiver functions in the LQT reference frame for station RAE. Here red indicates a positive phases. (a) shows the same stack as figure $2.16 \mathrm{~d}$ ) for the ZRT reference frame. (b) and (c) show Q stacks for receiver functions calculated using the LQT reference frame with (b) rotating for a top layer velocity of $3.5 \mathrm{~km} / \mathrm{s}$ and (c) rotating for a top layer velocity of $6.0 \mathrm{~km} / \mathrm{s}$. The LQT rotations show only minor improvement in the coherency of the arrival at $4.3 \mathrm{~s}$. 

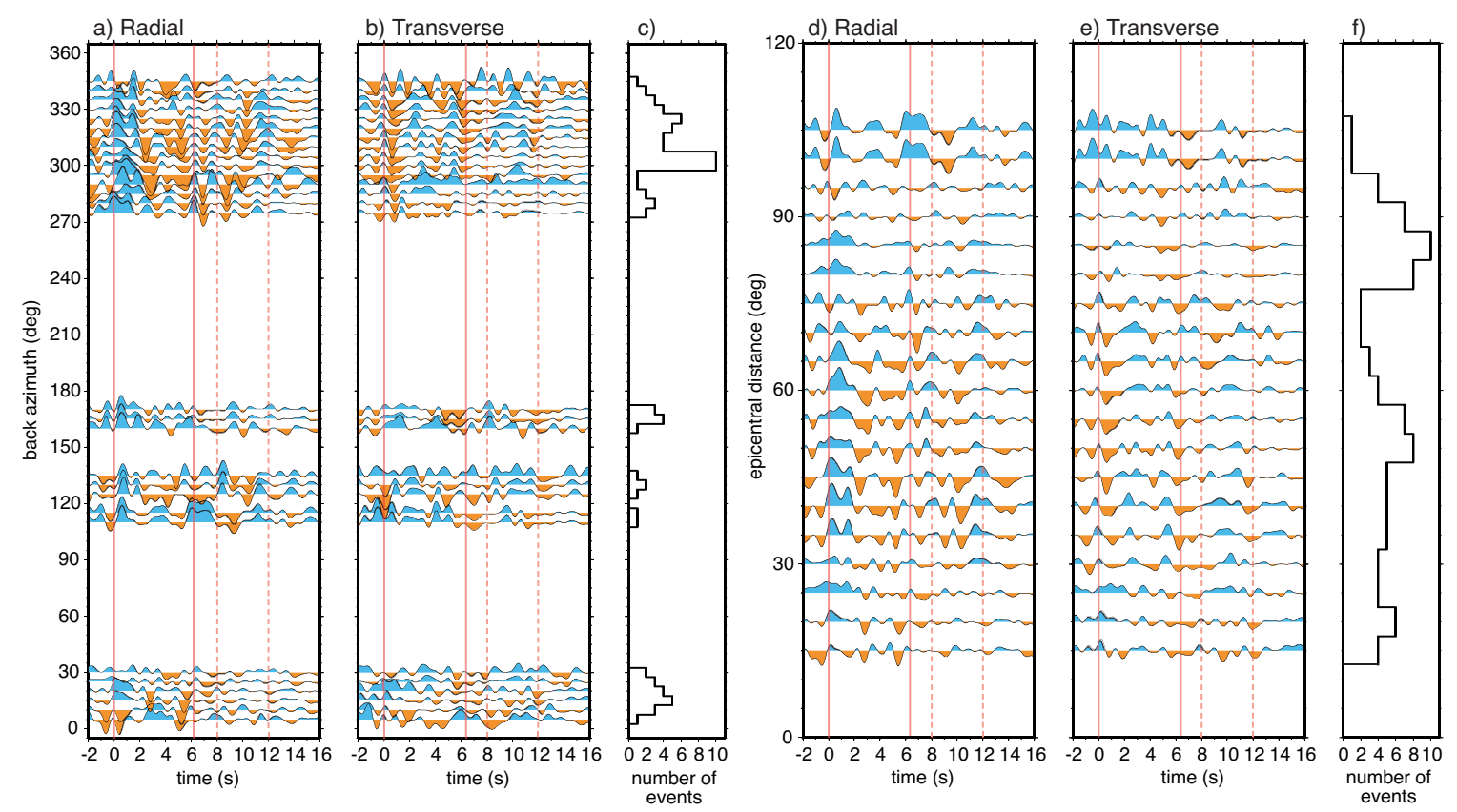

Figure 2.18: PAP receiver functions. Blue indicates a positive phase and red indicates a negative phase. (a) and (b) show $\mathrm{R}$ and $\mathrm{T}$ receiver functions stacked in back-azimuth bins. (d) and (e) show $\mathrm{R}$ and $\mathrm{T}$ receiver functions stacked in epicentral distance bins. (c) and (f) show the number of events in each stacking bin for back-azimuth and epicentral distance stacks respectively. The solid red lines are at $0 \mathrm{~s}$ and $6.3 \mathrm{~s}$ delay times. Dashed red lines are at $8 \mathrm{~s}$ and $12 \mathrm{~s}$
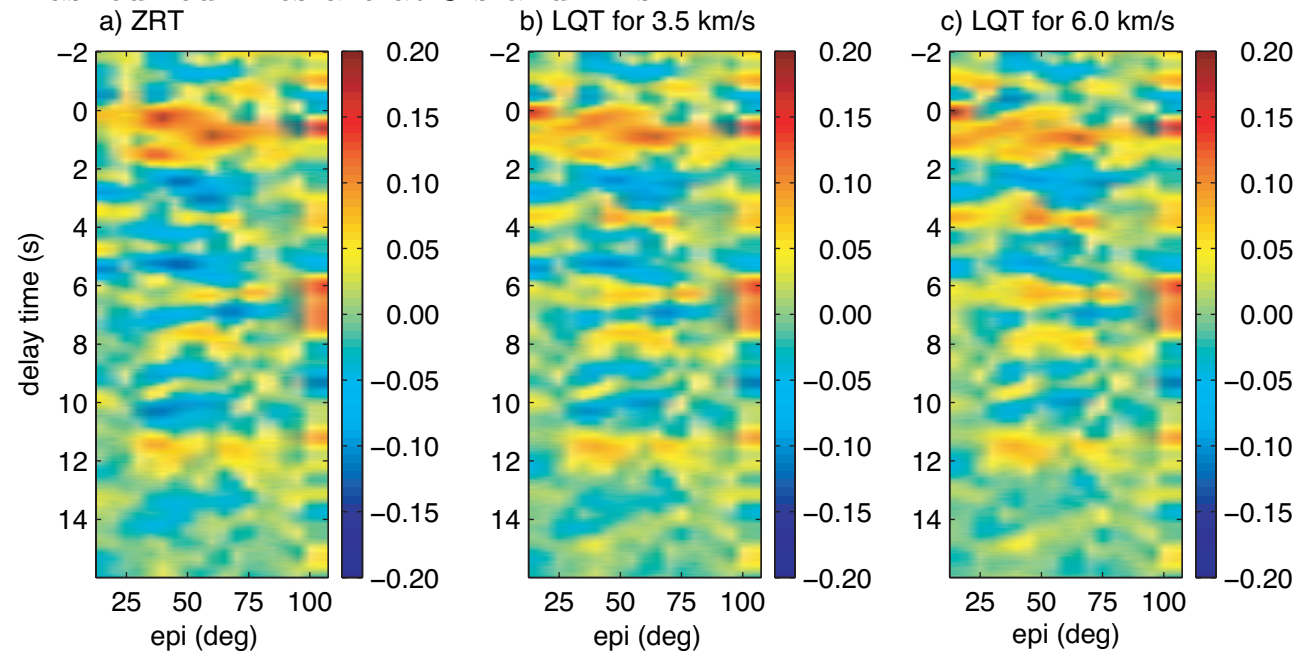

Figure 2.19: Epicentral distance stacked receiver functions in the LQT reference frame for station PAP. Here red indicates a positive phases. (a) shows the same stack as figure $2.18 \mathrm{~d}$ ) for the ZRT reference frame. (b) and (c) show Q stacks for receiver functions calculated using the LQT reference frame with (b) rotating for a top layer velocity of $3.5 \mathrm{~km} / \mathrm{s}$ and (c) rotating for a top layer velocity of $6.0 \mathrm{~km} / \mathrm{s}$. 


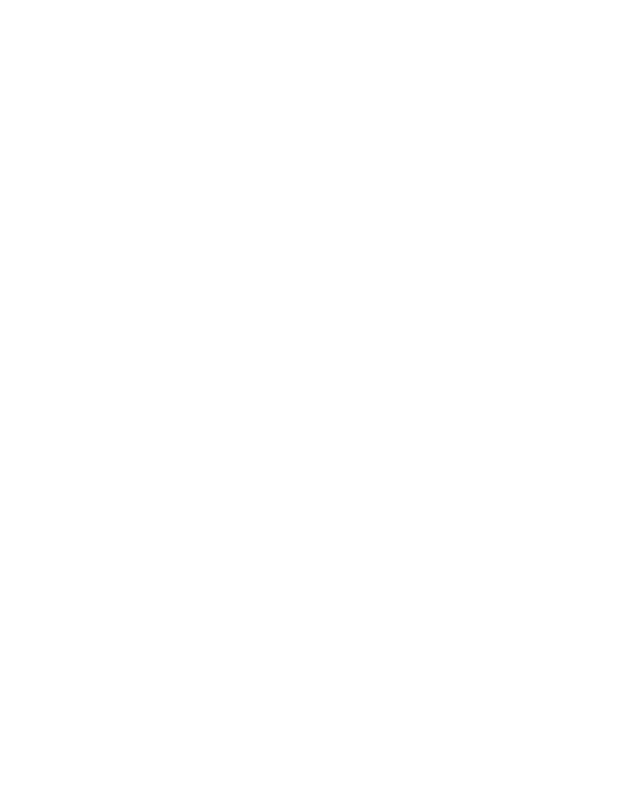

Figure 2.20: SUN receiver functions. Blue indicates a positive phases and red indicates a negative phases. (a) and (b) show $\mathrm{R}$ and $\mathrm{T}$ receiver functions stacked in back-azimuth bins. (d) and (e) show $\mathrm{R}$ and $\mathrm{T}$ receiver functions stacked in epicentral distance bins. (c) and (f) show the number of events in each stacking bin for back-azimuth and epicentral distance stacks respectively. The solid red line is at 0 delay time and the dashed red lines are at 8 and $13 \mathrm{~s}$ delay time.
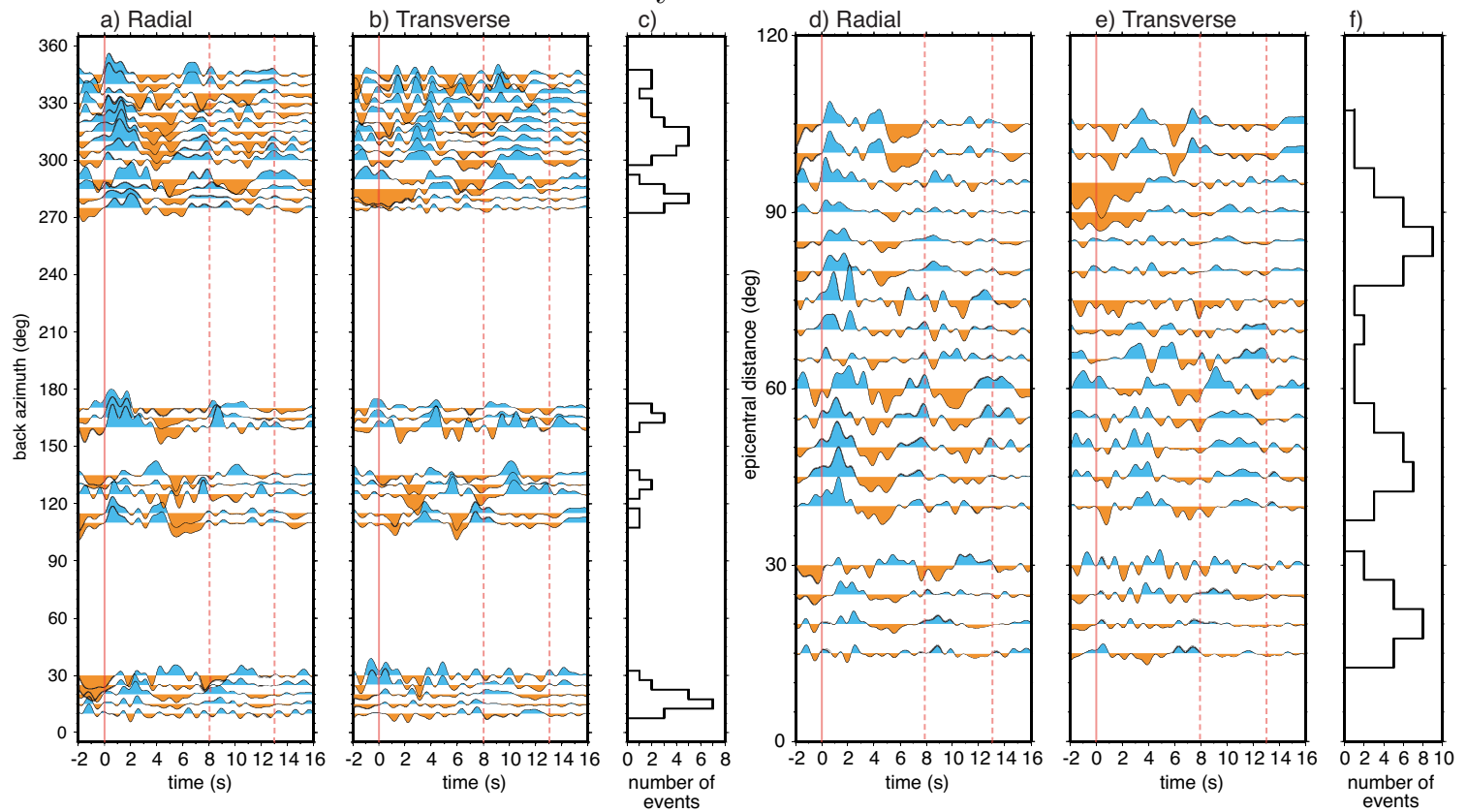

Figure 2.21: WRR receiver functions. Blue indicates a positive phase and red indicates a negative phase. (a) and (b) show $\mathrm{R}$ and $\mathrm{T}$ components stacked in back-azimuth bins. (d) and (e) show R and T components stacked in epicentral distance bins. (c) and (f) show the number of events in each stacking bin for back-azimuth and epicentral distance stacks respectively. The solid red line is at 0 delay time and the dashed red lines are at 8 and 13 s delay time. 
S.

Peaks in the transverse receiver functions for these two stations are also difficult to trace between stacking bins. Two positive peaks are observed on the transverse receiver functions of station SUN. The first, at $\sim 2.1 \mathrm{~s}$, is strongest for epicentral distances between $40^{\circ}$ and $75^{\circ}$. The second is at $\sim 10.6 \mathrm{~s}$ and is strongest for back-azimuths between $290^{\circ}$ and $315^{\circ}$. Rotation to LQT has little effect on these two stations and is not shown.

\section{TRAP data summary}

From the above, six common features can be identified on the 3 northern stations of the TRAP array:

- The first arrival is offset from a 0 time delay expected for a Pp arrival.

- A strong negative phase follows the first arrival.

- A positive phase can be seen between 3 and $3.4 \mathrm{~s}$ delay times. Delay times for this arrival gradually increase southward.

- A negative phase occurs between 5 and $6 \mathrm{~s}$.

- A positive phase is present at $\sim 8 \mathrm{~s}$ with decreasing delay times as epicentral distance increases.

- A positive phase is present at $\sim 12 \mathrm{~s}$ with increasing delay times as epicentral distance increases.

At station RAE these same features can be seen, but there is a $0.9 \mathrm{~s}$ increase in the delay time for the 3-3.4 s positive arrival seen to the north. South of RAE this arrival is no longer seen on the receiver functions. Receiver functions for the southern sites are characterised by a long period signal.

\subsubsection{GEONET stations}

\section{HIZ}

Station HIZ had 218 events that could be used for receiver function analysis. Radial receiver functions have a positive peak at $0 \mathrm{~s}$ delay time followed by another at 1 $\mathrm{s}$ (figure 2.22). The $0 \mathrm{~s}$ peak has a high amplitude that increases with decreasing epicentral distance as would be expect for a Pp arrival. Two more positive peaks are observed on the radial components, a strong peak at $2.6 \mathrm{~s}$ and a weaker peak at $4.7 \mathrm{~s}$. Receiver functions at short epicentral distances $\left(<20^{\circ}\right)$ have positive amplitudes out to $\sim 10 \mathrm{~s}$. 

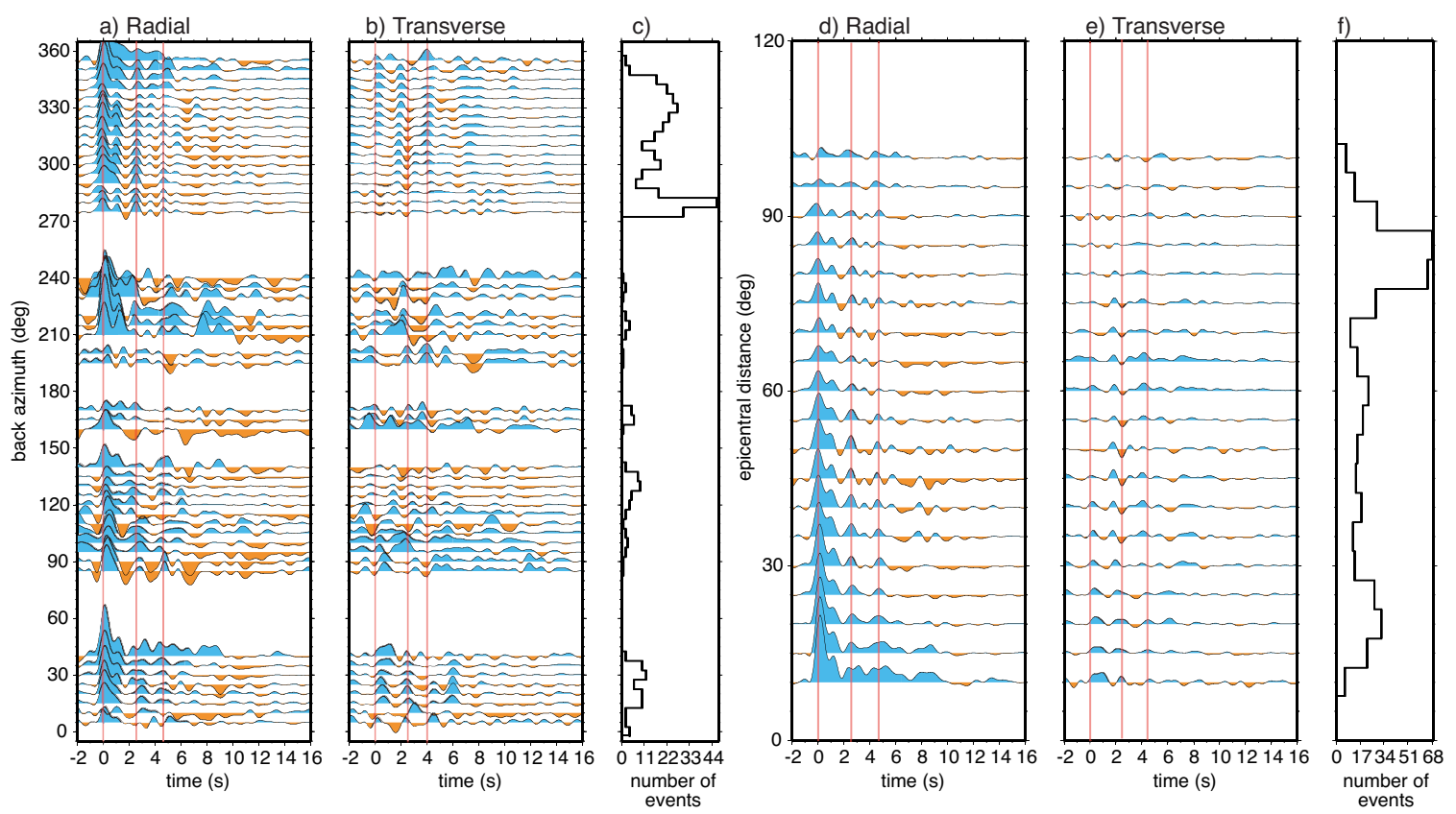

Figure 2.22: HIZ receiver functions. Blue indicates a positive phase and red indicates a negative phase. (a) and (b) show $\mathrm{R}$ and $\mathrm{T}$ receiver functions stacked in back-azimuth bins. (d) and (e) show $\mathrm{R}$ and $\mathrm{T}$ receiver functions stacked in epicentral distance bins. (c) and (f) show the number of events in each stacking bin. The solid red lines are at $0,2.6$ and 4.7 s delay times.

a) ZRT

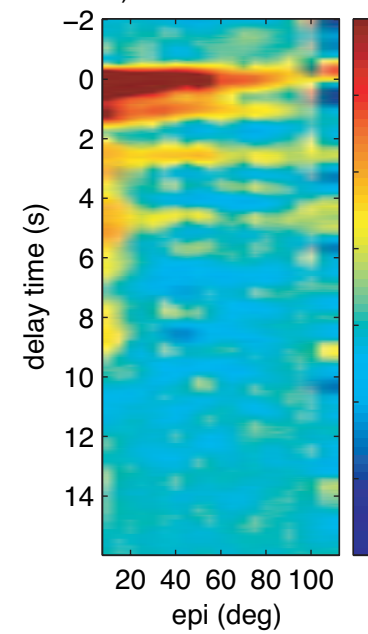

b) LQT for $3.5 \mathrm{~km} / \mathrm{s}$

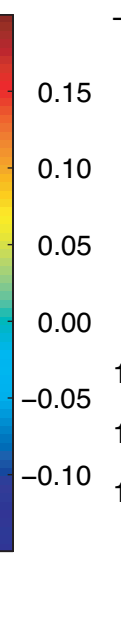

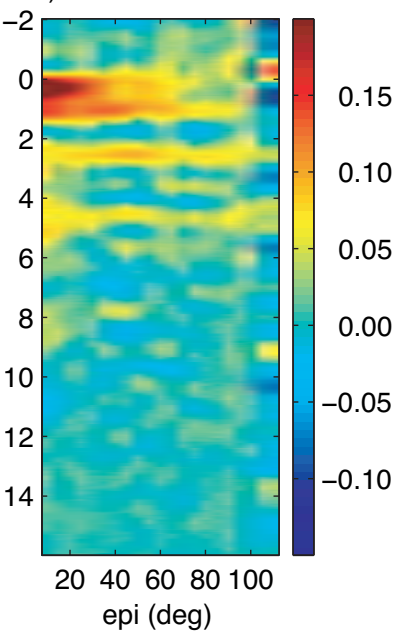

c) LQT for $6.0 \mathrm{~km} / \mathrm{s}$

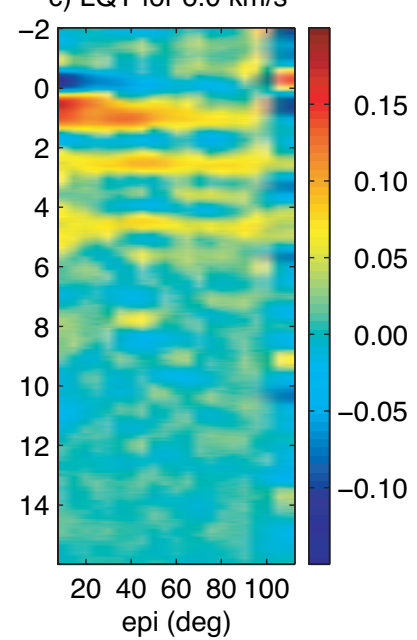

Figure 2.23: Epicentral distance stacked receiver functions in the LQT reference frame for station HIZ. Here red indicates a positive phases. (a) shows the same stack as figure $2.22 \mathrm{~d}$ ) for the ZRT reference frame. (b) and (c) show Q stacks for receiver functions calculated using the LQT reference frame with (b) rotating for a top layer velocity of $3.5 \mathrm{~km} / \mathrm{s}$ and (c) rotating for a top layer velocity of $6.0 \mathrm{~km} / \mathrm{s}$. 
There is much less energy on the transverse receiver functions than the radial. Three positive peaks are observed on the transverse receiver functions at $0.3 \mathrm{~s}, 2.0 \mathrm{~s}$ and 4.2 $\mathrm{s}$. The $2.0 \mathrm{~s}$ peak precedes a negative peak with the same timing as the positive arrival at $2.6 \mathrm{~s}$ on the radial component.

Rotation into the LQT reference frame reduces the energy at $0 \mathrm{~s}$ and enhances the $1 \mathrm{~s}$, $2.6 \mathrm{~s}$ and $4.7 \mathrm{~s}$ arrivals (figure 2.23) for receiver functions binned according to epicentral distance.

\section{VRZ}

Station VRZ recorded 202 events that could be used for receiver function analysis. Radial receiver functions have a band of positive energy out to approximately $2.0 \mathrm{~s}$ (figure 2.24). A weak positive peak is observed at $3.2 \mathrm{~s}$ and is followed by a stronger positive peak at $4.4 \mathrm{~s}$. There is little coherent energy on the radial receiver functions past the $4.4 \mathrm{~s}$ phase. Receiver functions at short epicentral distances $\left(<20^{\circ}\right)$ have positive amplitudes out to $\sim 8 \mathrm{~s}$ and there is a very weak positive phase just after $12 \mathrm{~s}$ for epicentral distances $>45^{\circ}$.

There is only a small amount of energy on the transverse receiver functions. The small arrival at $0 \mathrm{~s}$ delay time flips in polarity from positive, for northern back azimuths, to negative for southern back azimuths. This phase is followed by a phase with opposite polarity at $\sim 0.7 \mathrm{~s}$. Since most of the events used are to the north of the station, a small positive arrival at $0 \mathrm{~s}$ is observed on the epicentral distance plot followed by a negative. There is also a positive peak at $2.5 \mathrm{~s}$ in the back-azimuth binned plot.

Rotation into LQT reduces the amplitude of energy at $0 \mathrm{~s}$ and enhances the $4.4 \mathrm{~s}$ phase on epicentral distance binned plots (figure 2.25)

\section{WAZ}

Station WAZ recorded 144 events that could be used in receiver function analysis. Radial receiver functions for this station are similar to those of TRAP stations SUN and WRR. There are no clear single peaks, instead the radial receiver functions are characterised by a region of positive energy from 0 to $\sim 3.6$ s followed by a region of negative energy out to between $7.5 \mathrm{~s}$ and $8.4 \mathrm{~s}$ delay times. Transverse receiver functions follow the same pattern as the radial receiver functions.

Rotation into the LQT reference frame narrows the bands of positive and negative energy (figure 2.27). Almost all of the negative energy beyond 5 s delay time is removed by rotating into this reference frame. This indicates that much of this energy is due to P multiples. 

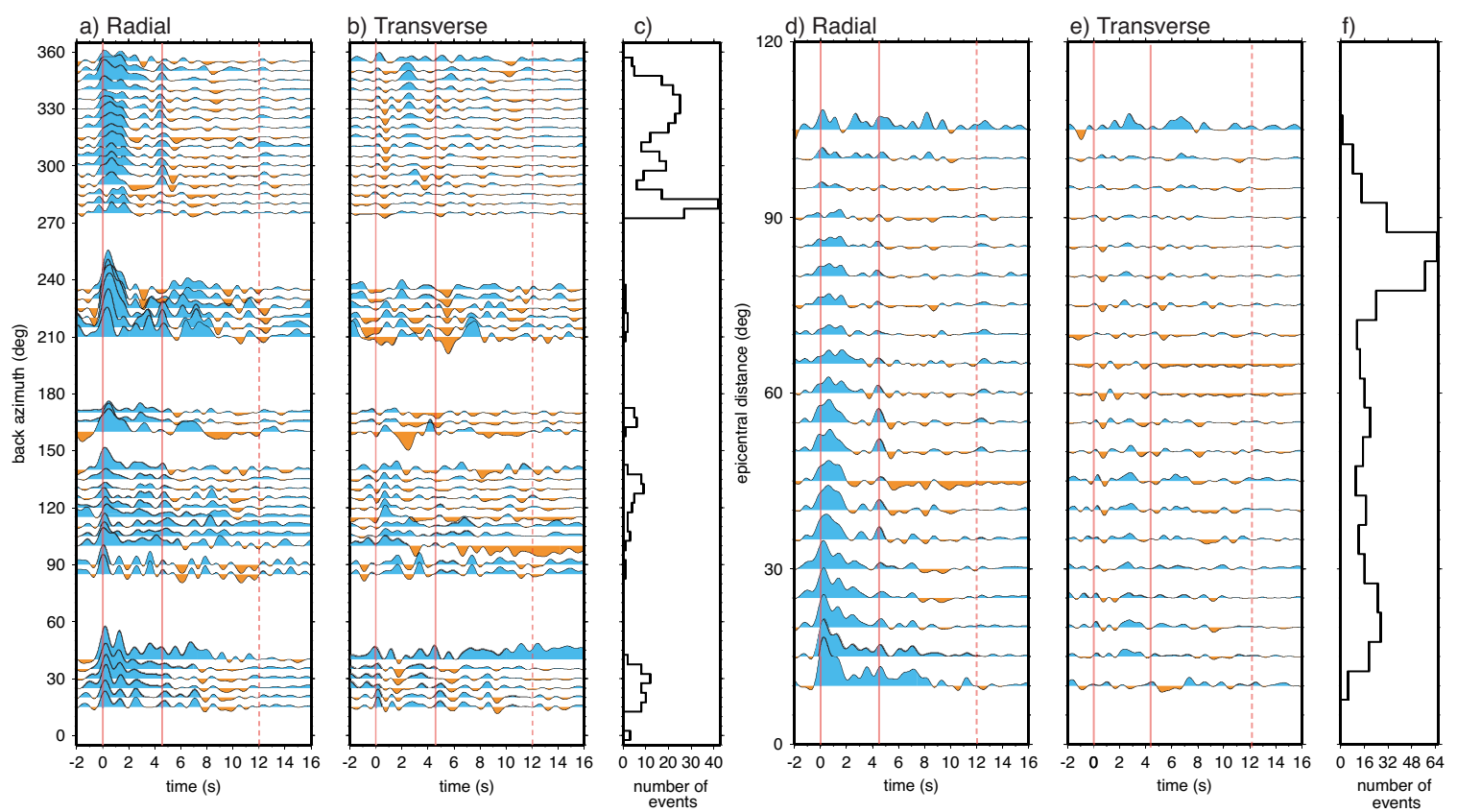

Figure 2.24: VRZ receiver functions. Blue indicates a positive phase and red indicates a negative phase. (a) and (b) show $R$ and $T$ receiver functions stacked in back-azimuth bins. (d) and (e) show $\mathrm{R}$ and $\mathrm{T}$ receiver functions stacked in epicentral distance bins. (c) and (f) show the number of events in each stacking bin. The solid red lines are at 0 and $4.4 \mathrm{~s}$ delay times and the dashed red line is at $12 \mathrm{~s}$ delay time.

a) ZRT

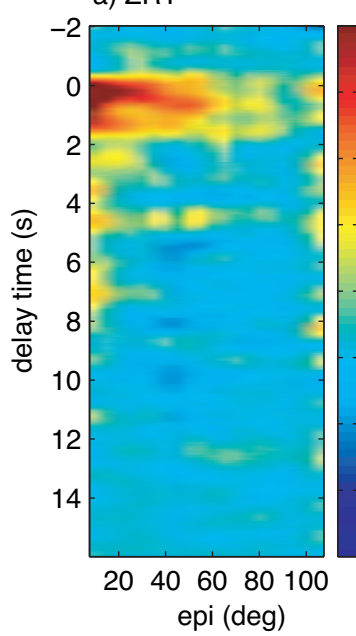

b) LQT for $3.5 \mathrm{~km} / \mathrm{s}$

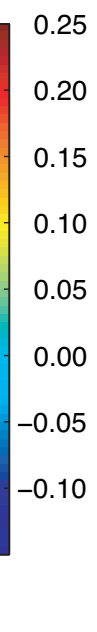

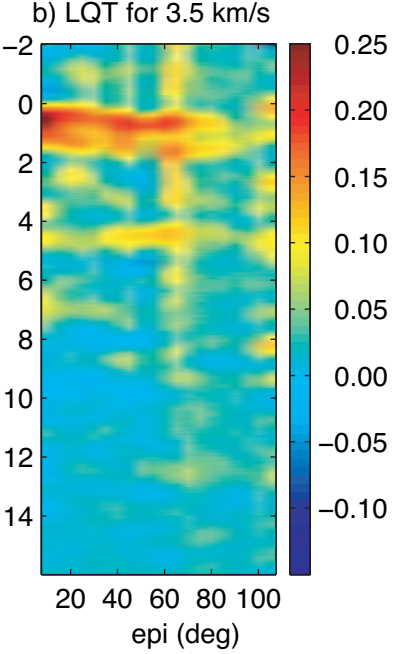

c) LQT for $6.0 \mathrm{~km} / \mathrm{s}$

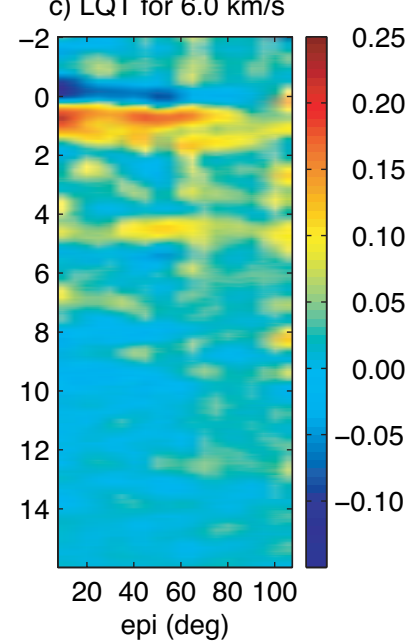

Figure 2.25: Epicentral distance stacked receiver functions in the LQT reference frame for station VRZ. Here red indicates a positive phase. (a) shows the same stack as figure $2.24 \mathrm{~d}$ ) for the ZRT reference frame. (b) and (c) show Q stacks for receiver functions calculated using the LQT reference frame with (b) rotating for a top layer velocity of $3.5 \mathrm{~km} / \mathrm{s}$ and (c) rotating for a top layer velocity of $6.0 \mathrm{~km} / \mathrm{s}$. 

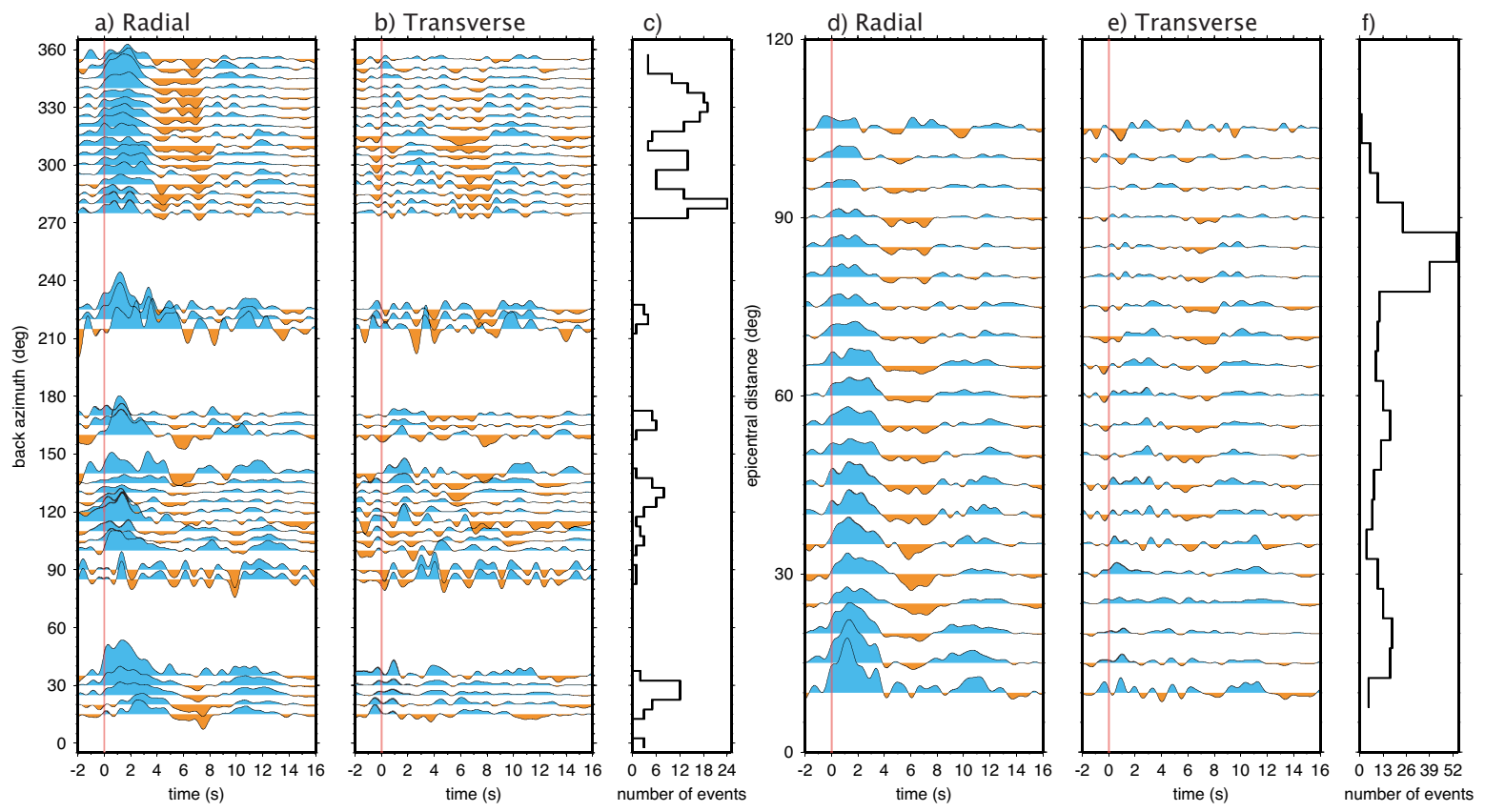

Figure 2.26: WAZ receiver functions. Blue indicates a positive phase and red indicates a negative phase. (a) and (b) show $\mathrm{R}$ and $\mathrm{T}$ receiver functions stacked in back-azimuth bins. (d) and (e) show $\mathrm{R}$ and $\mathrm{T}$ receiver functions stacked in epicentral distance bins. (c) and (f) show the number of events in each stacking bin. The solid red line indicates the $0 \mathrm{~s}$ delay time.

a) ZRT

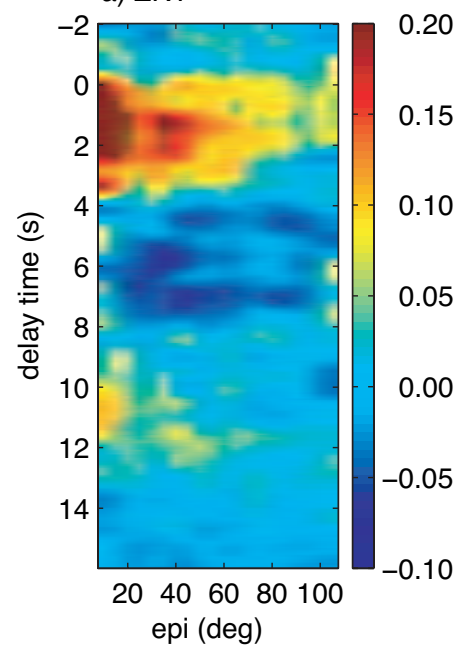

b) LQT for $3.5 \mathrm{~km} / \mathrm{s}$

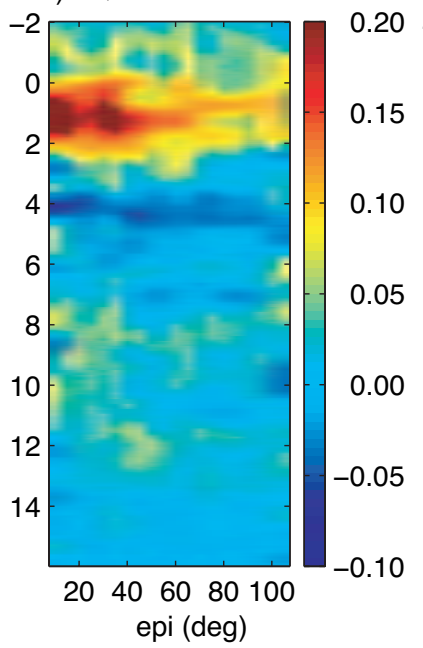

c) LQT for $6.0 \mathrm{~km} / \mathrm{s}$

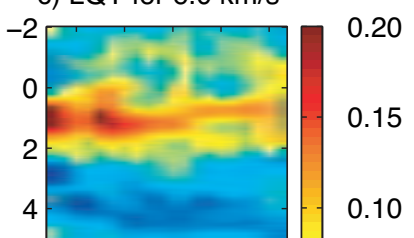

0.05

0.00

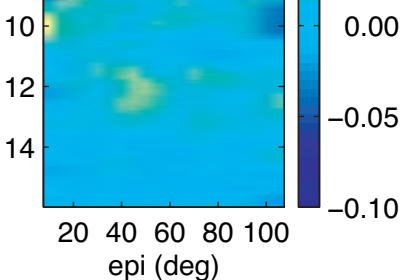

Figure 2.27: Epicentral distance stacked receiver functions in the LQT reference frame for station WAZ. Here red indicates a positive phase. (a) shows the same stack as figure $2.26 \mathrm{~d}$ ) for the ZRT reference frame. (b) and (c) show Q stacks for receiver functions calculated using the LQT reference frame with (b) rotating for a top layer velocity of $3.5 \mathrm{~km} / \mathrm{s}$ and (c) rotating for a top layer velocity of $6.0 \mathrm{~km} / \mathrm{s}$. 


\subsubsection{CNIPSE stations}

\section{YUPC}

Station YUPC is situated just west of the Central Volcanic Region. For this station 60 events could be used for receiver function analysis. Radial receiver functions have a positive peak at $0 \mathrm{~s}$ (figure 2.28), which has an increasing amplitude with decreasing epicentral distance as expected for the Pp arrival. A positive peak at $2.7 \mathrm{~s}$ is preceded by a negative peak. A smaller positive peak can be seen at $\sim 4.1 \mathrm{~s}$ and is immediately followed by a negative at $4.8 \mathrm{~s}$. A larger negative peak is observed at $\sim 6.7 \mathrm{~s}$. Delay times for this phase increase with increasing epicentral distance.
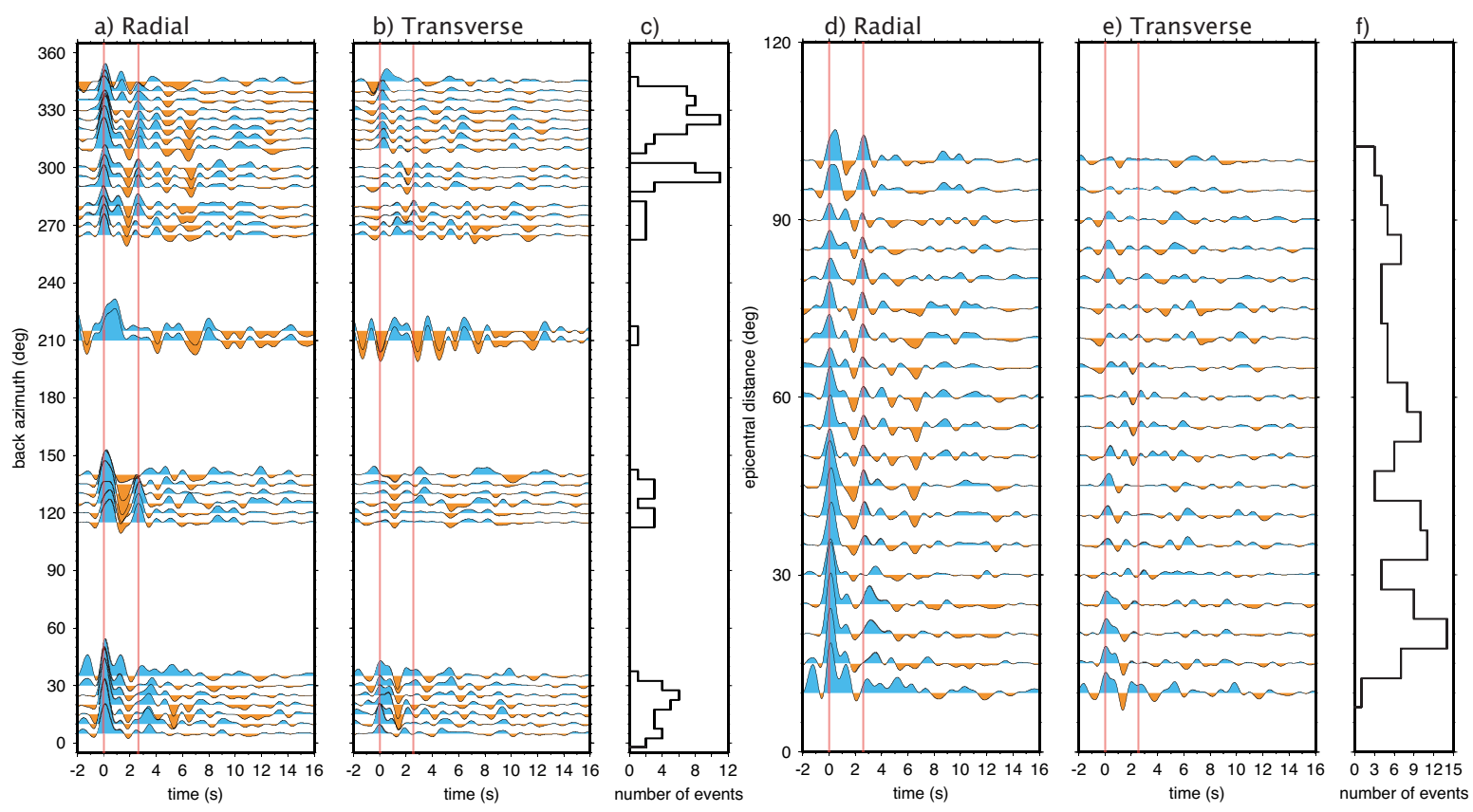

Figure 2.28: YUPC receiver functions. Blue indicates a positivephase and red indicates a negative phase. (a) and (b) show $\mathrm{R}$ and $\mathrm{T}$ receiver functions stacked in back-azimuth bins. (d) and (e) show $\mathrm{R}$ and $\mathrm{T}$ receiver fucntions stacked in epicentral distance bins. (c) and (f) show the number of events in each stacking bin. The solid red lines are at $0 \mathrm{~s}$ and $2.7 \mathrm{~s}$ delay times.

Transverse receiver functions have a strong positive arrival at just after the 0 delay time for back-azimuths ranges $300^{\circ}-340^{\circ}$ and $0^{\circ}-35^{\circ}$. There is also a negative peak just before $2 \mathrm{~s}$. This peak is particularly strong for the $0^{\circ}-35^{\circ}$ back-azimuth range.

\section{MILC}

Station MILC recorded 31 events that could be used for receiver function analysis. On the radial receiver functions a strong peak is observed at $0 \mathrm{~s}$ delay time (figure 2.29). This peak has an increasing amplitude with decreasing epicentral distance as expected for a Pp arrival. There is a positive peak at $\sim 3.2 \mathrm{~s}$ for the $265^{\circ}-340^{\circ}$ back-azimuth range. At other back-azimuths there are positive arrivals just before and just after this time. The transverse receiver functions are very noisy for this station and show little 
coherent energy.
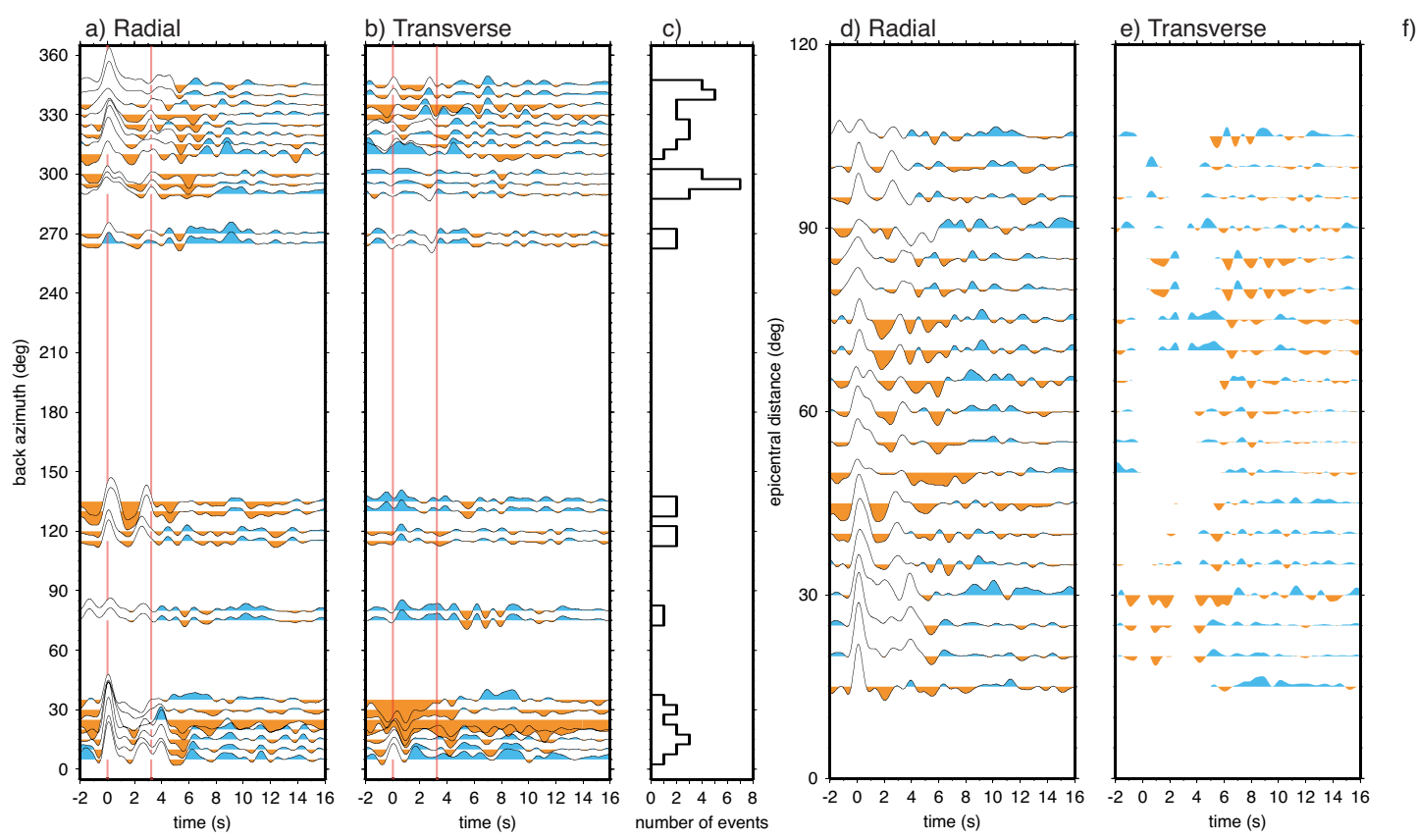

Figure 2.29: MILC receiver functions. Blue indicates a positive phase and red indicates a negative phase. (a) and (b) show $\mathrm{R}$ and $\mathrm{T}$ receiver fucntions stacked in back-azimuth bins. (d) and (e) show $\mathrm{R}$ and $\mathrm{T}$ receiver fucntions stacked in epicentral distance bins. (c) and (f) show the number of events in each stacking bin. The solid red lines are at $0 \mathrm{~s}$ and $3.2 \mathrm{~s}$ delay times

\subsection{Interpretation}

\subsubsection{Piercing points}

While receiver functions use events with raypaths that have a near vertical incidence angle, there is a horizontal offset between where the ray pierces a boundary and the station. This offset distance is dependent on the ray parameter, $p$, the depth of the boundary $(z)$, and the wave speed, $c$, between the boundary of interest and the receiver. The location where a raypath pierces a boundary (the piercing point) can be estimated using Snell's law (equation 2.3.1). For a given depth $z$ the horizontal offset $x$ of the piercing point for the Ps conversion is given by:

$$
x=\frac{z V_{s} p}{\sqrt{1-V_{s}^{2} p^{2}}}
$$

where $V_{s}$ is average shear wave velocity between the boundary and the receiver. The location of Moho piercing points have been estimated for each station assuming a 30 $\mathrm{km}$ thick crust, a $V_{s}$ of $3.54 \mathrm{~km} / \mathrm{s}$ and horizontal slowness parameters estimated from the AK135 travel time curves (figure 2.30). 


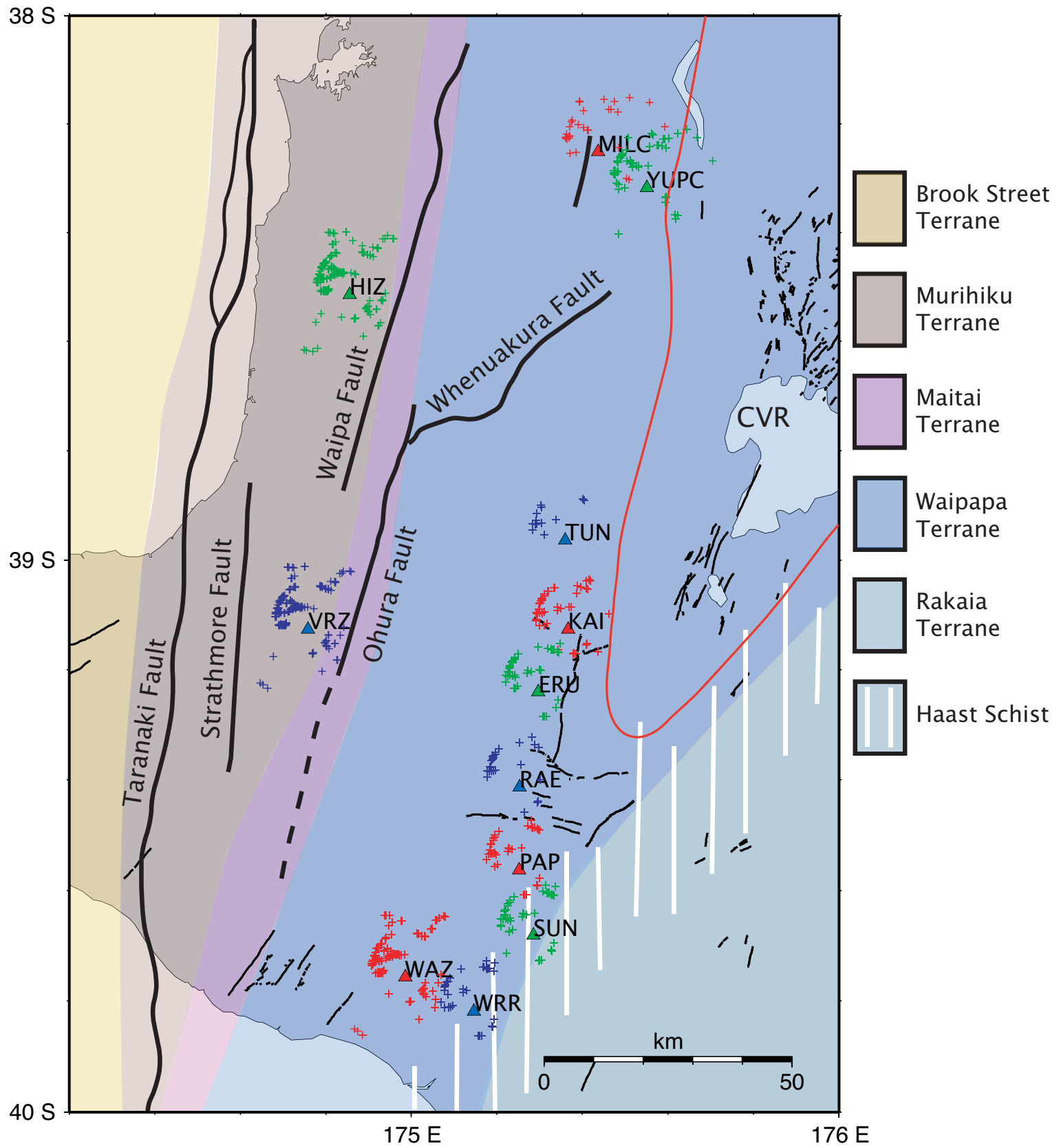

Figure 2.30: Piercing points at $30 \mathrm{~km}$ depth. Estimated piercing points for each station are shown as red, green and blue crosses. Colours are alternated according to station so that piercing points for each station can be differentiated from each other. Basement terranes and tectonic overprinting are indicated as per the key. Thick black lines indicate large scale crustal faults and thin black lines indicate active faulting [Edbrooke 2005]. The red line indicates the boundary of the Central Volcanic Region. Triangles indicate station locations 
Piercing point offsets for Ps conversions at $30 \mathrm{~km}$ depth range from $4.3 \mathrm{~km}$ to 14.6 $\mathrm{km}$. The lateral distance from the station sampled by the PpPs multiple can also be estimated using:

$$
x=z\left(\frac{V_{s} p}{\sqrt{1-V_{s}^{2} p^{2}}}+2 \frac{V_{p} p}{\sqrt{1-V_{p}^{2} p^{2}}}\right)
$$

where $V_{p}$ is the average $\mathrm{P}$ wave velocity between the boundary and the receiver. Assuming a $V_{p}$ of $6.1 \mathrm{~km} / \mathrm{s}$ and a boundary depth of $30 \mathrm{~km}$, the distance the PpPs multiple samples is up to $65 \mathrm{~km}$ from the receiver.

Another consideration is the radius of the Fresnel zone. Teleseismic waves can be approximated as plane waves. For this approximation the radius (R) of the 1st Fresnel zone is:

$$
\begin{aligned}
R & =\sqrt{\left(z+\frac{\lambda}{2}\right)^{2}-z^{2}} \\
& \approx \sqrt{z \lambda}
\end{aligned}
$$

where $z$ is the depth of the interface and $\lambda$ is the wavelength of the converted $\mathrm{S}$ wave [Sheriff and Geldart 1995]. For an S wave velocity of $3.54 \mathrm{~km} / \mathrm{s}$ (average crustal S wave velocity for the New Zealand Standard velocity model [Maunder 2002]) and a frequency of $1 \mathrm{~Hz}$, the Fresnel zone radius is $\sim 10 \mathrm{~km}$ for a depth of $30 \mathrm{~km}$.

\subsubsection{Moho arrivals}

The New Zealand standard velocity model [Maunder 2002](table 2.2) gives an average crustal $V_{p}$ of $6.1 \mathrm{~km} / \mathrm{s}$ and $V s$ of $3.54 \mathrm{~km} / \mathrm{s}$. Using these velocities, Ps conversions from the Moho would be expected at delay times between 2.5 and $5.0 \mathrm{~s}$ for depths between 20 and $40 \mathrm{~km}$. All but the southern four sites, WRR, WAZ, SUN and PAP, have a positive arrival in this time window, which is interpreted as the Moho arrival. Delay times for the Moho arrival generally increase from north to south.

Two stations have two positive arrivals in the expected time window (HIZ and YUPC). In the case of station HIZ, there are positive arrivals as $2.6 \mathrm{~s}$ and $4.7 \mathrm{~s}$ delay times (figures 2.22 and 2.23). Here the earlier arrival is favoured as the Moho conversion. This arrival has higher amplitudes than the $4.7 \mathrm{~s}$ arrival. HIZ is situated on basement rock and is therefore not effected by the low velocities of near surface sediments. If the general trend of decreasing delay time to the north is followed, the Moho conversion would be expected at delay times of less than $3.3 \mathrm{~s}$. Combined with the absence of sediments it is reasonable to expect this site to have small delay times for the Moho conversion. Data from HIZ were used as part of the local earthquake study of Sherburn 
and White [2005], which shows that this site is characterised by high $\mathrm{P}$ and $\mathrm{S}$ wave velocities. Reyners et al. [2006] also show that this region has low $V_{p} / V_{s}$ ratios $(<1.75)$, which would have the effect of decreasing delay times.

HIZ is positioned close to two large scale faults, the Taranaki fault $\sim 25 \mathrm{~km}$ to the west and the Waipa fault $\sim 10 \mathrm{~km}$ to the east (figure 2.30). Large scale faults can act as scatterers [Abers 1998], producing phases that are coherent with the Pp arrival. Station corrections for a local earthquake study in the Taranaki region indicate that there is a change in near surface $\mathrm{P}$ and $\mathrm{S}$ wave velocity across the Taranaki fault [Sherburn and White 2005], making this a potential source of near surface $\mathrm{P}$ to $\mathrm{S}$ conversions. This station is also in close proximity the Maitai terrane, a narrow band of ultramafic basement rock (also known as the Junction Magnetic Anomaly). This constitutes a change in the nature of basement rocks and may also be a source of crustal scatter. The small amplitude $4.7 \mathrm{~s}$ arrival may therefore be the result of near surface conversions from faults or geological boundaries.

Station YUPC has positive arrivals at $2.7 \mathrm{~s}$ and $4.1 \mathrm{~s}$ (figure 2.28). As with HIZ the earlier arrival is favoured as the Moho conversion. This is once again the higher amplitude arrival and this station is also situated on basement rock. As part of the analysis by Bannister et al. [2007], receiver gathers were calculated for CNIPSE stations west of the Central Volcanic Region (including MILC and YUPC). They show delay times for the Moho conversion decrease smoothly between MILC and YUPC from $\sim 3.3 \mathrm{~s}$ at MILC to $\sim 2.5 \mathrm{~s}$ for raypaths west of YUPC. They also show the results for a receiver function inversion for station YUPC which indicate a crust-mantle boundary at $\sim 20-25 \mathrm{~km}$.

Two stations, VRZ and RAE, have delay times that do not follow the trend of gradually increasing delay time southward. For station VRZ the prefered Moho arrival is at $4.4 \mathrm{~s}$ delay time (figure 2.24 and 2.25). Station VRZ is at approximately the same latitude as KAI (figure 2.30) but there is a $1 \mathrm{~s}$ increase in the delay time of the Moho arrival between these two stations. Both KAI and VRZ have approximately the same thickness of sediments below the station (table 2.1) so this can be ruled out as cause for the increase in delay time. A $1 \mathrm{~s}$ change in delay time would require an $8 \mathrm{~km}$ change in crustal thickness between the stations or a $10-15 \%$ increase in $V_{p} / V_{s}$. An increase in crustal thickness of this magnitude should be evident in the isostatic gravity anomaly, however there is little variation in the isostatic gravity anomaly between these two stations (figure 1.2). VRZ is situated in an area associated with high crustal $V_{p} / V_{s}$ (> 1.75) [Reyners et al. 2006], close to the Maitai terrane and within the region of the Junction Magnetic Anomaly[Mortimer et al. 1997]. This region is associated with the serpentinized Dun Mountain ophiolote belt [Mortimer et al. 1997]. Serpentinite has a very high $V_{p} / V_{s}$ ratio ( $>2.0$ ) [Christensen 1996]. Where the contact between the Murihuki and Maitai terranes is exposed in the South Island, the two terranes are 
commonly faulted [Coombs et al. 1976]. There are three large scale faults (Taranaki and Strathmore to the west and Ohura to the east)(figure 2.30) close to VRZ. Major crustal faulting is also associated with high $V_{p} / V_{s}$ ratios [Thurber et al. 2003]. The combination of faulting and the presence of serpentine could produce the required $10-15 \%$ increase in $V_{p} / V_{s}$.

At RAE the Moho conversion arrives at $4.3 \mathrm{~s}$ (figure 2.16 and 2.17), $0.9 \mathrm{~s}$ later than the station immediately to the north (ERU). Receiver functions for this station sample the same basement terrane as the stations immediately to the north (figure 2.30), there are no large scale faults in the vicinity and Reyners et al. [2006] show no significant increase in $V_{p} / V_{s}$ ratio in this region. A change in crustal thickness is therefore proposed as the origin of the high delay time at station RAE. This station is close to a steep slope in the isostatic gravity anomaly, which has been modeled as a change in crustal thickness of the order of $10 \mathrm{~km}$ [Stern et al. 1987]. This is approximately compatible with the increase in delay time seen at RAE.

Along with delay time there is also an increase in sediment depth from north to south (figure 2.4 and table2.1). Typically $V_{p} / V_{s}$ ratios for sediments are higher than those for crystalline basement. For orogenic belts such as New Zealand a representative $V_{p} / V_{s}$ ratio for the crust is 1.73 [Zandt and Ammon 1995] and for Wanganui basin sediments $V_{p} / V_{s}$ is $>1.8$ [Eberhart-Phillips et al. 2005]. For these $V_{p} / V_{s}$ ratios an increase in sediment thickness will result in an increase in delay time. To account for sediment thickness a delay time correction is calculated using equation 2.2.1, where $H_{s}$ is set to the sediment thickness below the station. The expected delay time is calculated for the sediment layer using a $V_{p}$ of $3.4 \mathrm{~km} / \mathrm{s}$ [Watson and Allen 1964] and $V_{p} / V_{s}$ of 1.85. The difference between this delay time and the delay time calculated using the average crustal velocity of the NZST velocity model (table 2.2) for the same $H_{s}$ value gives a time correction $\left(\Delta t_{P s}\right.$ sed equation 2.5.4) for each station. This calculation of station corrections is relatively insensitive to the $V_{p}$ values used, but very sensitive to the $V_{p} / V_{s}$ ratios. Station elevation also varies between stations, ranging from $700 \mathrm{~m}$ to near sea level. A correction for elevation $\left(\Delta t_{P s} e l e v\right)$ is calculated using equation 2.2.1, where $H_{e}$ is the elevation of the receiver.

$$
\begin{aligned}
& \Delta t_{P s} s e d=t_{P s}\left(H_{s}\right) \text { sediment }-t_{P s}\left(H_{s}\right) \text { crust } \\
& \Delta t_{P s} \text { elev }=t_{P s}\left(H_{e}\right) \text { crust }
\end{aligned}
$$

Sediment corrections vary from 0 for stations with no sediment to $0.37 \mathrm{~s}$ where the sediments are deepest. Elevation corrections are small $(<0.1 \mathrm{~s})$ in comparison to sediment corrections. Figure 2.31 shows profiles of elevation, sediment depth and Moho delay times along the line from stations WRR in the south to MILC in the north. Even 
after taking into account the variation in delay times caused by elevation and sediment thickness there is still a $0.8 \mathrm{~s}$ increase in delay time for the Moho arrival between stations ERU and RAE. This is equivalent to $\sim 6.5 \mathrm{~km}$ increase in crustal thickness assuming $V_{p} / V_{s}$ of 1.73 .

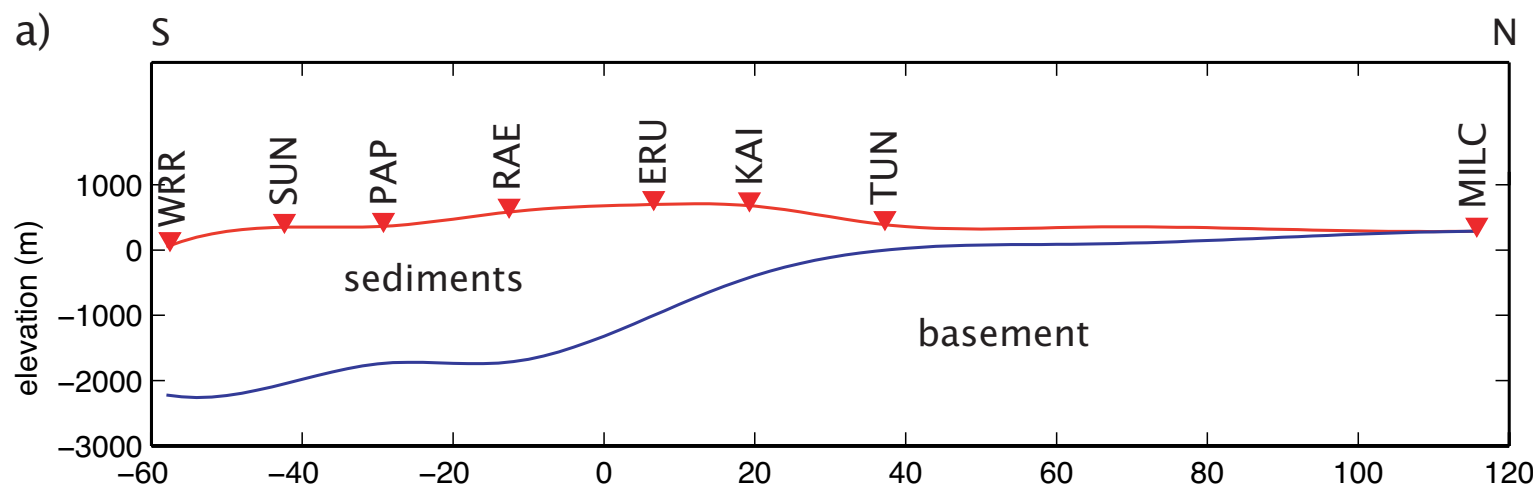

b)

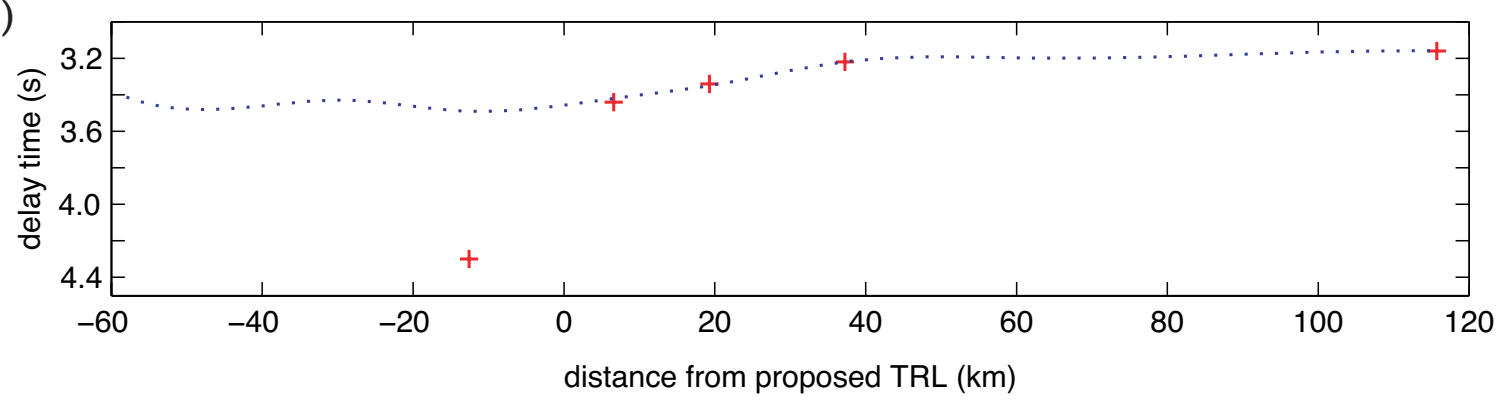

Figure 2.31: Expected delay times for a flat Moho. a) A profile through stations WRR to MILC showing the station elevation (solid red line) and the sediment depth (solid blue line). Stations along the profile are shown as inverted triangles. b) Delay times for Moho arrivals (red crosses). Expected variation in Moho delay times given the sediment depth and elevation from a) for a flat Moho are shown as a dotted line. Note that delay times for station WRR, SUN and PAP are not shown since no Moho arrival could be seen in the receiver functions for these individual stations.

At station KAI there is a strong Moho multiple. Multiples can be identified as phases where delay time increases with epicentral distance. The multiple identified at station KAI has arrival times between 10 and $12 \mathrm{~s}$ (figure 2.13). The presence of multiples can provide additional constraints on crustal thickness and geometry (see 2.5.3).

Mantle conversions In addition to the Moho arrivals, four stations (KAI, ERU, RAE and PAP) show a positive arrival at a delay time of $\sim 8 \mathrm{~s}$. The delay time for this arrival decreases with increasing epicentral distance, which is indicative of a Ps conversion. An $8 \mathrm{~s}$ delay time puts the boundary at which this conversion occurs within the mantle at approximately $65 \mathrm{~km}$ depth. 


\subsection{3 $H-V_{p} / V_{s}$ stacking}

Receiver functions provide time delays between the direct $\mathrm{P}$ arrival and Ps converted phases from seismic boundaries. Equation 2.5.6 can be used to translate these delay times to depth $(H)$.

$$
H=\frac{t_{P s}}{\sqrt{\frac{1}{V_{s}^{2}}-p^{2}}-\sqrt{\frac{1}{V_{p}^{2}}-p^{2}}}
$$

This equation requires both a $V_{p}$ and $V_{s}$ velocity model. While the depth calculation is not very sensitive to the absolute velocity model, it is highly dependent on the $V_{p} / V_{s}$ ratio. Zhu and Kanamori [2000] show that a $0.1 \mathrm{~km} / \mathrm{s}$ error in $V_{p}$ will result in $\sim 0.5$ $\mathrm{km}$ error in depth while a 0.1 error in $V_{p} / V_{s}$ will result in $\sim 4 \mathrm{~km}$ error in depth.

The ambiguity between $H$ and $V_{p} / V_{s}$ can be reduced if the additional information provided by multiples is used (equations 2.5.7 and 2.5.8).

$$
\begin{aligned}
& H=\frac{t_{P p P s}}{\sqrt{\frac{1}{V_{s}^{2}}-p^{2}}+\sqrt{\frac{1}{V_{p}^{2}}-p^{2}}} \\
& H=\frac{t_{P p S s+P s P s}}{2 \sqrt{\frac{1}{V_{s}^{2}}-p^{2}}}
\end{aligned}
$$

The $t_{P p S s+P s P s}$ phase, which should arrive after the $t_{P p P s}$ multiple and have a negative polarity, cannot be identified in any of the receiver function stacks (figures 2.11-2.29). If present this arrival would be close to the maximum limit in reliable receiver function retrieval $(16 \mathrm{~s})$. However for a given $V_{p}$ model the $t_{P p P s}$ multiple is sufficient to solve for $H$ (equation 2.5.7). One disadvantage of using multiples to constrain the depth of a boundary is that the multiples sample a much larger lateral area than the direct Ps conversion. For a $30 \mathrm{~km}$ Moho the piercing point of the Ps conversion typically occurs within $10 \mathrm{~km}$ (laterally) of the station, while the PpPs phase samples $\sim 5$ times this distance (figure 2.2). Consequently the use of multiples to determine $H$ will smooth any lateral variability over this distance.

In practice picking Ps and PpPs phase arrivals on individual receiver functions is often difficult with real data, therefore the method of Zhu and Kanamori [2000] has been used to stack receiver functions in the $H-V_{p} / V_{s}$ domain. The amplitudes of individual receiver functions are stacked at predicted phase arrival times for $H-V_{p} / V_{s}$ pairs for a given $V_{p}$. The Ps and PpPs phases have been stacked using equation 2.5.9.

$$
s(H, V p / V s)=\sum_{n=1}^{N} w_{1} r_{n}\left(t_{1}\right)+w_{2} r_{n}\left(t_{2}\right)
$$


where $s$ is the stacked amplitude in the $H-V_{p} / V_{s}$ domain, $N$ is the number of receiver functions stacked, $r_{n}(t)$ is the amplitude of the $n$th radial receiver function (or $\mathrm{Q}$ receiver function where the LQT reference frame is used) at time $t, t_{1}$ and $t_{2}$ are the predicted arrival times of the Ps and PpPs phases respectively and $w_{1}$ and $w_{2}$ are weighting factors for each phase $\left(\sum w_{i}=1\right)$. Where $s\left(H, V_{p} / V_{s}\right)$ reaches a maximum the two phases are stacked coherently, giving a best fit value for both $H$ and $V_{p} / V_{s}$. Errors have been calculated by a boot-strap method that uses 200 randomly selected sample populations of receiver functions to determine the $95 \%$ confidence interval.

This method of stacking is only appropriate where there is little lateral variability in $H$ or $V_{p} / V_{s}$. Where this is not the case, phases will not stack coherently. Therefore this method has not been used where the path of the PpPs multiple crosses the proposed TRL. Since most of the events used in this study come from the north, use of this method is restricted to stations north of the proposed TRL. Lateral variation in sediment thickness will only have a small impact on the $V_{p} / V_{s}$ ratio $(1 \mathrm{~km}$ increase in sediment thickness approximately translates to a 0.01 increase in $V_{p} / V_{s}$ ratio). Of the stations north of the proposed TRL, multiples can be clearly identified at station KAI (figure 2.13) making it ideal for $H-V_{p} / V_{s}$ stacking.

Since only two phases are considered here, each is given equal weighting $\left(w_{i}=0.5\right)$. Stacked amplitudes, $s\left(H, V_{p} / V_{s}\right)$, are calculated using equation 2.5.9 at increments of $0.2 \mathrm{~km}$ in $H$ and 0.01 in $V_{p} / V_{s}$. The $H$ range is restricted to $15-45 \mathrm{~km}$ (reasonable extremes for crustal thickness in the area) and $V_{p} / V_{s}$ from 1.5 to 2.0. Initially $V_{p}$ is set to an average crustal velocity of $6.0 \mathrm{~km} / \mathrm{s}$ calculated from the New Zealand Standard model with the addition of a $1 \mathrm{~km}$ low-velocity $(3.4 \mathrm{~km} / \mathrm{s})$ layer at the surface. Receiver functions that have been rotated to the LQT reference frame using a velocity of 6.0 $\mathrm{km} / \mathrm{s}$ are used since this reference frame accentuates the Ps and PpPs arrivals (figure 2.13). Figure 2.32a shows the stacking results for these parameters. Two lines of positive stacking amplitude are observed. These are the contributions to the stack of the two phases; the steeper line corresponds to the PpPs multiple and the other corresponds to the Ps arrival. Where the two phases intersect, the stacking amplitude reaches a maximum. Stacking returns an $H$ of $26.4 \pm 1.5 \mathrm{~km}$ and a $V_{p} / V_{s}$ of $1.72 \pm 0.09$.

For a region west of this study Sherburn and White [2005] derive a 1D crustal velocity model from a local earthquake study that has much lower upper crustal velocities than the standard New Zealand velocity model. Controlled source data [Stratford and Stern 2006] also indicates that the average crustal velocity of the northwestern North Island may be significantly lower that that of the standard New Zealand velocity model. An average crustal $V_{p}$ of $5.8 \mathrm{~km} / \mathrm{s}$ is calculated from the velocity model of Stratford and Stern [2006]. Figure 2.32b shows the stacking results using an average crustal $V_{p}$ of $5.8 \mathrm{~km} / \mathrm{s}$. With this crustal $V_{p}$, stacking returns an $H$ of $25.2 \pm 1.5 \mathrm{~km}$ and 

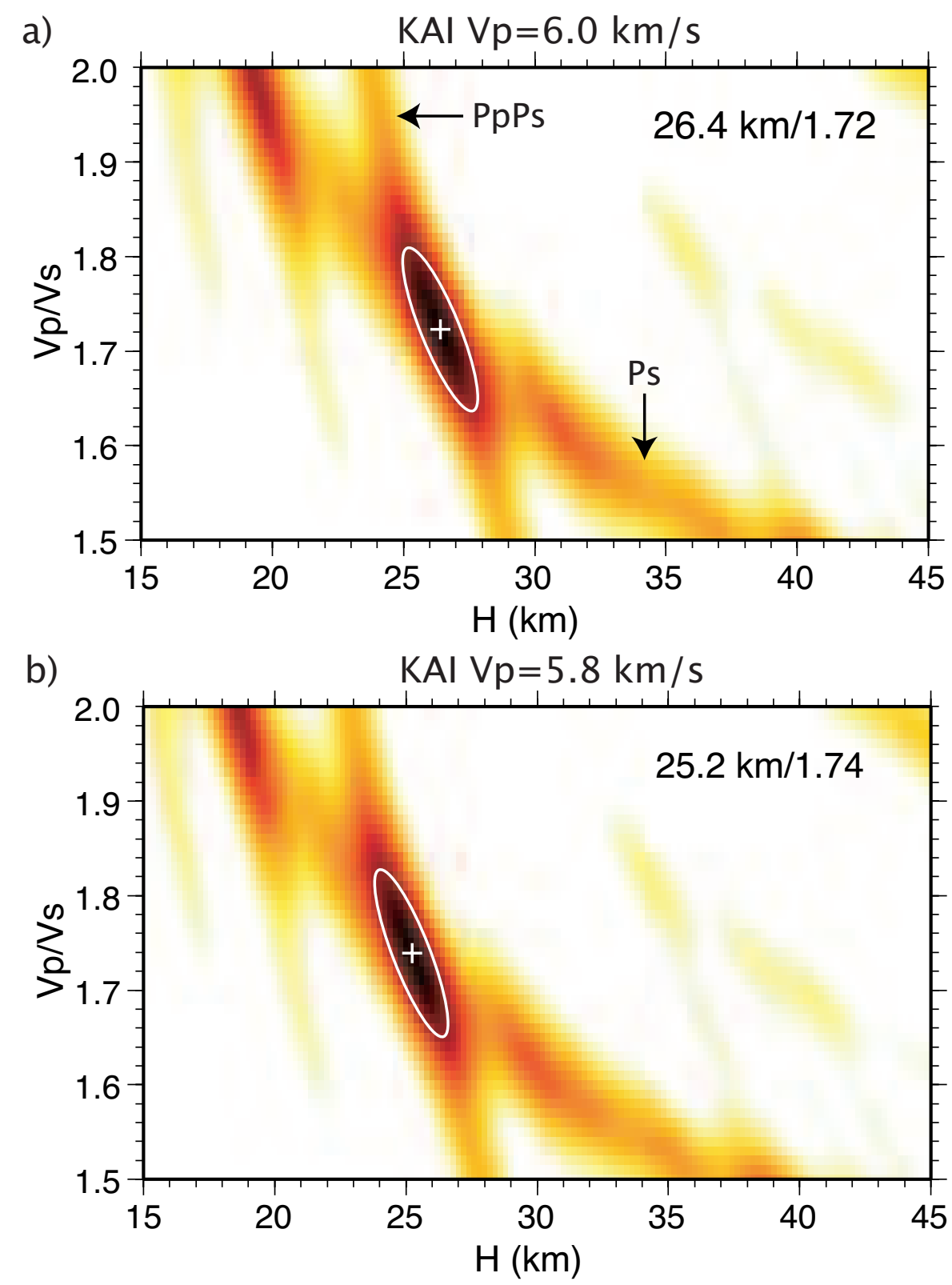

Figure 2.32: $H-V_{p} / V_{s}$ stack results for station KAI. a) shows results using a crustal $V_{p}$ of $6.0 \mathrm{~km} / \mathrm{s}$. The contributions from the Ps and PpPs phases are labeled. b) shows results using a crustal $V_{p}$ of $5.8 \mathrm{~km} / \mathrm{s}$. Darker red indicates higher stacking amplitude. The white cross indicates the peak amplitude and the white ellipse encompasses the $95 \%$ confidence region. For $V_{p}=6.0 \mathrm{~km} / \mathrm{s}$ the peak amplitude occurs at $H=26.4 \mathrm{~km}$, $V_{p} / V_{s}=1.72$. For $V_{p}=5.8 \mathrm{~km} / \mathrm{s} H=25.2 \mathrm{~km}, V_{p} / V_{s}=1.74$ 
$V_{p} / V_{s} 1.74 \pm 0.09$. The stacking results using the two different crustal $V_{p}$ values have overlapping uncertainties and are both in good agreement with the crustal thicknesses derived from a controlled source line to the north which range from $25 \mathrm{~km}$ [Harrison and White 2004] to $26 \pm 2 \mathrm{~km}$ [Stratford and Stern 2006].

Although the remaining TRAP stations north of the TRL do not have clear multiple arrivals, the information from station KAI can be used to estimate a crustal thickness. Ps arrivals from stations MILC, TUN, KAI and ERU range between 3.16-3.44 s. Without taking into account elevation or sediment thickness, this gives a depth range 23.6-25.9 km (using $V_{p} / V_{s} 1.74$ ) or 24.9-27.4 (using $V_{p} / V_{s} 1.72$ ). By using corrections for sediment thickness and elevation, this range can be reduced. From station ERU northward a crustal thickness of $25 \pm 1.5 \mathrm{~km}$ is observed. If the same $V_{p} / V_{s}$ ratios are applied to station RAE, a crustal thickness of $32 \mathrm{~km}$ is returned. This suggests there is a $7 \mathrm{~km}$ increase in crustal thickness between stations ERU and RAE.

The GEONET station, VRZ, has what appears to be a very weak multiple, which arrives just after $12 \mathrm{~s}$ delay time (figures 2.24-2.25). Since this station has an anomalously late Moho arrival, an $H-V_{p} / V_{s}$ stack is performed on its receiver functions. For this station the $V_{p} / V_{s}$ range is shifted upward, to include high $V_{p} / V_{s}$ ratios expected for serpentinite. The $H$ range is also limited to $16-45 \mathrm{~km}$, since positive energy out to $2 \mathrm{~s}$ causes a high in stacking amplitude at low $V_{p} / V_{s}$ and shallow depths.

Figure 2.33 shows stacking results for station VRZ. Unlike the stacking results at station KAI the multiple phase is not clear on the stacking plot. Peak stacking amplitudes occur at $\mathrm{H}$ values of $25 \pm 2 \mathrm{~km}$ and $24.1 \pm 2 \mathrm{~km}$ for $V_{p}$ values of $6.0 \mathrm{~km} / \mathrm{s}$ and $5.8 \mathrm{~km} / \mathrm{s}$ respectively. These depths are consistent with those at station KAI but $V_{p} / V_{s}$ ratios are much higher $(2.05 \pm 0.12)$.

\subsubsection{CCP gathers and depth migration}

$H-V_{p} / V_{s}$ stacking provides an average crustal thickness and $V_{p} / V_{s}$ ratio, over an area with a radius of up to $65 \mathrm{~km}$, under the assumption that the structure sampled by $\mathrm{P}_{m} \mathrm{~s}$ and $\mathrm{PpP}_{m} \mathrm{~s}$ is one-dimensional. To study the lateral variations in subsurface features, common conversion point (CCP) stacking is used. This is a technique similar to common mid point stacking used in reflection studies, but predicted conversion points are used instead of source receiver midpoints. This method also has the advantage that coherent noise in the receiver functions from a single station, such as sediment reverberation, often becomes incoherent in multiple stations stacks [Dueker and Sheehan 1998].

The geographical stacking method of Dueker and Sheehan [1997; 1998] has been used to make CCP gathers for stations in the TRAP array. Receiver functions are first 

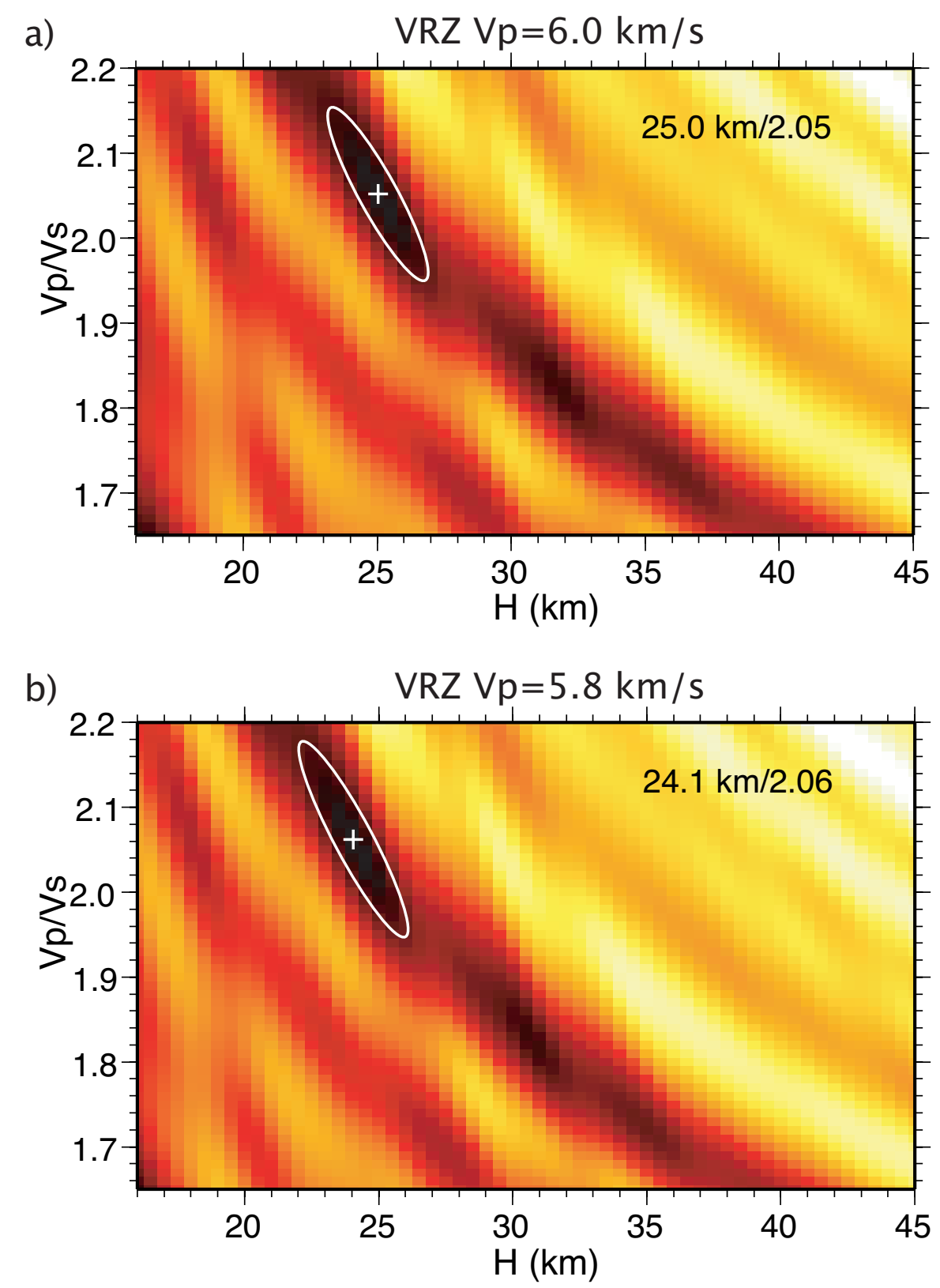

Figure 2.33: $H-V_{p} / V_{s}$ stack results for station VRZ. a) shows results using a crustal $V_{p}$ of $6.0 \mathrm{~km} / \mathrm{s}$ and b) shows results using a crustal $V_{p}$ of $5.8 \mathrm{~km} / \mathrm{s}$. Darker red indicates higher stacking amplitude. The white cross indicates the peak amplitude and the white ellipse encompasses the $95 \%$ confidence region. For $V_{p}=6.0 \mathrm{~km} / \mathrm{s}$ the peak amplitude occurs at $H=25 \mathrm{~km}, V_{p} / V_{s}=2.05$. For $V_{p}=5.8 \mathrm{~km} / \mathrm{s} H=24.1 \mathrm{~km}, V_{p} / V_{s}=2.06$ 
bandpass filtered using a zero-phase second order butterworth filter with corner frequencies of 0.2 and $1.5 \mathrm{~Hz}$. This helps reduce the amplitude of long period sediment reverberations. A station correction for sediment depth and station elevation is then applied as per equations 2.5.4 and 2.5.5. These are the only lateral variation corrections made for this data since tomographic modeling indicates there is little variation in crustal $V_{p} / V_{s}$ ratios below $4 \mathrm{~km}$ along the TRAP array [Reyners et al. 2006]. $\mathrm{P}_{d} \mathrm{~s}$ ( $\mathrm{P}$ to $\mathrm{S}$ conversion at depth $\mathrm{d}$ ) piercing points are found by ray tracing the converted $\mathrm{S}$ wave raypaths using a 1-D velocity model and equation 2.3.1. Piercing points are geographically binned using a 2-D vertical grid of sample points. Receiver functions are mapped along their ray paths traced in 3 dimensions through a flat-layered velocity model using equation 2.5.10 which corrects for event-station moveout while at the same time performing a pre-stack depth migration. This step is performed using the same flat-layered velocity model used for ray tracing. All rays within a bin are summed to form a stacked trace for each horizontal bin, creating a 2-D depth section.

$$
T_{P_{d} s}(p)=\int_{-D}^{0}\left[\sqrt{\frac{1}{V_{s}(z)^{2}}-p^{2}}-\sqrt{\frac{1}{V_{p}(z)^{2}}-p^{2}}\right] d z
$$

The receiver functions used were calculated in the LQT reference frame using a $V_{p}$ velocity of $3.5 \mathrm{~km} / \mathrm{s}$ to help remove $\mathrm{P}$ wave sediment multiples. The NZST velocity model is used as an initial 1-D velocity model. This velocity model gives both $\mathrm{P}$ and $\mathrm{S}$ wave velocities. The crustal $V_{p} / V_{s}$ ratio of this model is 1.71 down to $25 \mathrm{~km}$, slightly lower than estimates from $H-V_{p} / V_{s}$ stacking, but within the uncertainties. Since receiver functions are corrected for the low $V_{p} / V_{s}$ sediments in the stacking procedure, the $V_{p} / V_{s}$ ratio is expected to be reduced from $H-V_{p} / V_{s}$ stacking (which includes the sediments) by up to 0.03 so the model $V_{p} / V_{s}$ ratio is acceptable. The only significant difference between this model and that of Stratford and Stern [2006] down to $25 \mathrm{~km}$ depth is the top $2 \mathrm{~km}$ of low velocity material, which has already been compensated for in the station corrections for sediment depth. For a Moho up to $25 \mathrm{~km}$ depth these two models are therefore nearly indistinguishable and for a deeper Moho the NZST velocity model is more appropriate.

Since the primary focus is the topography of the Moho, horizontal bin width $(x)$ is set to the estimated width of the 1st Fresnel zone for an interface at $30 \mathrm{~km}$ depth calculated using equation 2.5.3. Assuming a signal frequency of $1 \mathrm{~Hz}$ (frequency at which the $\cos ^{2}$ filter reaches 0.25 and also the natural frequency of the instruments used) and an $\mathrm{S}$ wave velocity of $3.5 \mathrm{~km} / \mathrm{s}$ sets bin widths to $21 \mathrm{~km}$. Horizontal bins are spaced at $7 \mathrm{~km}$ centres to provide a 3 bin spatial correlation. The vertical bin width is set to $1 \mathrm{~km}$ since this a good estimate of vertical resolution, which is approximated as $1 / 4$ of the minimum wavelength. 
The out of plane distance of raypaths has not been limited. For a north-south section through the array, the out of plane distance for a piercing point at $30 \mathrm{~km}$ depth is less than $20 \mathrm{~km}$ and for $70 \mathrm{~km}$ depth, piercing points are up to $30 \mathrm{~km}$ out of plane. For a boundary which dips out of plane the maximum dip, $\theta$, which will stack into the 2-D depth section coherently for the entire out of plane width can be calculated using $\theta=\arctan (\lambda / 2 y)$, where $y$ is the out of plane distance to the piercing point. For an $\mathrm{S}$ wave velocity of $3.54 \mathrm{~km} / \mathrm{s}$ and a signal frequency of $1 \mathrm{~Hz}$ this equates to a maximum dip of $\sim 5$ degrees.

Figure 2.34 shows the resulting image, in the depth-distance domain, for a south to north cross section along the TRAP array. Positive amplitudes are shown in red and negative amplitudes are shown in blue. Positive amplitudes indicate an increase in velocity with depth across the boundary and negative amplitudes indicate decreasing velocities with depth. The main focus here is on the Moho conversion labeled $P_{m} s$. In the north (distances $>0$ ) this is a prominent flat positive feature at around 25 $\mathrm{km}$ depth. This feature remains reasonably flat even when no correction for lateral variation is applied. There is a step in Moho depth between stations RAE and ERU. In the south the Moho conversion is deeper $(>30 \mathrm{~km})$, weakens and dips southward reaching $35 \mathrm{~km}$ depth below station SUN. The dipping section of the Moho is lower in amplitude than the northern section. Since most of the events used are updip of the stations, the drop in Moho amplitude for the dipping section is expected [e.g. Cassidy 1992].

A strong positive phase is seen immediately below the $\mathrm{P}_{m} \mathrm{~s}$ converter $(\sim 32 \mathrm{~km})$ at the northern end of the line below station TUN. This phase weakens but continues southward to station ERU, approximately paralleling the Moho conversion. At station KAI this phase corresponds to the positive peak seen at $\sim 5 \mathrm{~s}$ (figure 2.13). The moveout of this phase at station KAI suggests that it is a multiple from a midcrustal boundary at $10-15 \mathrm{~km}$ depth. South of station ERU a similar phase is seen.

Above $15 \mathrm{~km}$ the advantages of stacking are lost since above this the Fresnel zones of adjacent stations do not always overlap. Above $15 \mathrm{~km}$ the sediment reverberations dominate the section, as can be seen by the positive amplitudes in the top $5-12 \mathrm{~km}$. The depth corresponding to this positive phase increases with increasing sediment thickness (figure 2.31) and table 2.1). For the southern sites much of the long period reverberation has been reduced by the band pass filter used, but the northern stations show a deep negative phase immediately after the initial positive one, which is likely to be due to the sediments.

A strong positive conversion with lateral coherence is apparent below the Moho at $\sim 65 \mathrm{~km}$ depth (labeled $\mathrm{P}_{2} \mathrm{~s}$ ). $\mathrm{P}_{2} \mathrm{~S}$ is coherent between stations TUN and RAE but disappears south of station RAE. There are no multiples from this boundary with which 

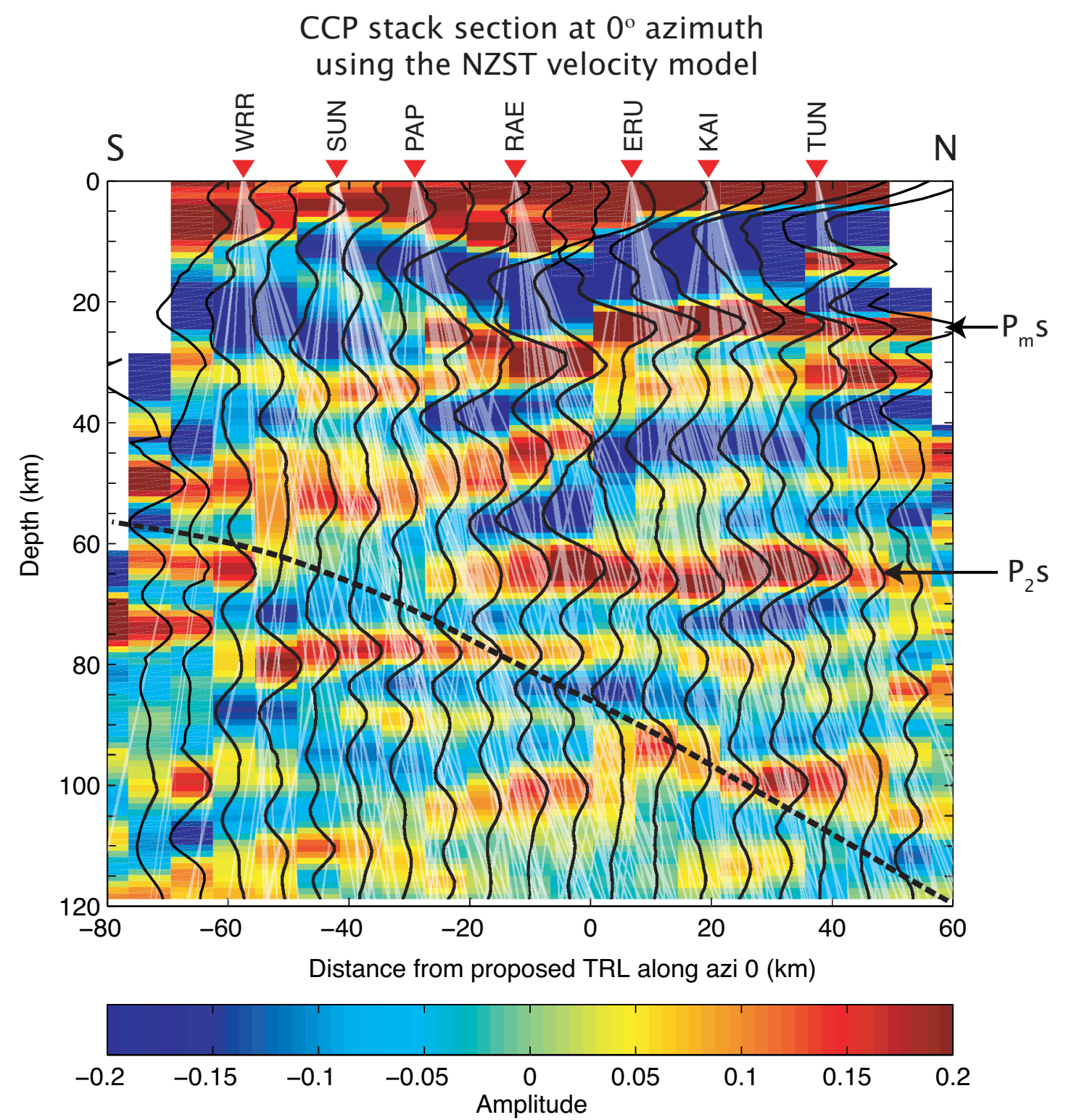

Figure 2.34: S-N CCP stack section for the TRAP array using the New Zealand standard crustal model. This section is oriented along the line of the TRAP array. Red colours indicate positive phases, blue colours indicate negative phases. Gathers have been corrected for sediment depth and elevation. Stacked traces are shown in black and in plane raypaths are shown in white. The Moho conversion is labeled $\mathrm{P}_{m} \mathrm{~s}$. This conversion is nearly flat north of station RAE. There is a step in Moho depth between stations RAE and ERU. South of RAE the moho conversion dips southward. The mantle conversion is labeled $\mathrm{P}_{2} \mathrm{~S}$. The dashed black line is the projected slab profile below this cross section [Ansell and Bannister 1996]. 


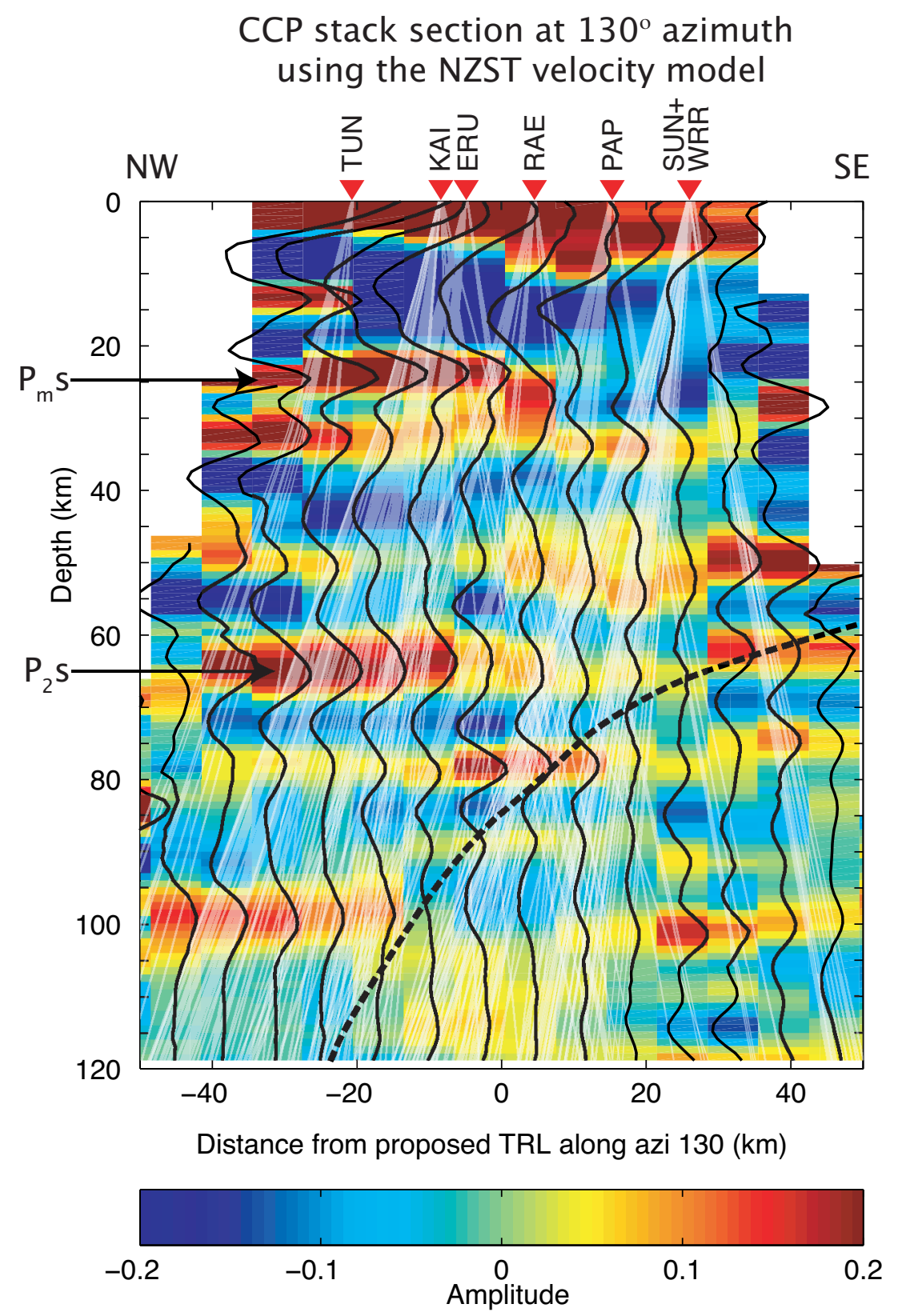

Figure 2.35: NW-SE CCP stack section for the TRAP array using the New Zealand standard crustal model. This section is oriented perpendicular to the strike of the subduction slab. Red colours indicate positive phases, blue colours indicate negative phases. Gathers have been corrected for sediment depth and elevation. Stacked traces are shown in black and in plane raypaths are shown in white. The Moho conversion is labeled $\mathrm{P}_{m} \mathrm{~s}$. This conversion is nearly flat north of station RAE. There is a step in Moho depth between stations RAE and ERU. South of RAE the moho conversion dips southward. The mantle conversion is labeled $\mathrm{P}_{2} \mathrm{~s}$. The dashed black line is the projected slab profile below this cross section [Ansell and Bannister 1996]. 
to constrain the $V_{p} / V_{s}$ ratio and depth, however the error in depth can be estimated by using the NZST velocity model down to $25 \mathrm{~km}$ depth and varying the $V_{p} / V_{s}$ ratio for the mantle between 1.7 and 1.8 and the mantle velocity between 7.2 and 8.2. Within these bounds the depth to the $\mathrm{P}_{2} \mathrm{~S}$ boundary is $65 \pm 5 \mathrm{~km}$ and approximately coincides with an increase in $V_{p} / V_{s}$ ratio seen in tomographic images [Reyners et al. 2006] and with an increase in seismic attenuation (see chapter 3 ). This conversion is above the expected projection of the subducting slab [Ansell and Bannister 1996], however to confirm that this is not a slab conversion a CCP stack has been performed for a cross section perpendicular to the strike of the slab (section azimuth is $130^{\circ}$ figure 2.35). The $\mathrm{P}_{2} \mathrm{~s}$ feature remains near horizontal in this projection, confirming that it is not a slab conversion. This boundary appears to be truncated close to the projection of the slab.

Figure 2.36a shows receiver functions for all the stations north of the TRL stacked in ray parameter $(\mathrm{p})$ bins. Bin widths are $0.09 \mathrm{~s} / \mathrm{km}$ wide and are spaced at $0.03 \mathrm{~s} / \mathrm{km}$ centres. Receiver functions have been corrected for sediment thickness and elevation prior to stacking. A prominent positive phase can be seen at $\sim 3.1 \mathrm{~s}$, which corresponds a NZST velocity model depth of $25 \mathrm{~km}$. The mantle phase seen on the CCP stacks is less obvious in this plot but can be seen as a positive phase between 7 and $8 \mathrm{~s}$. The Moho multiple arrives slightly after the moveout curve predicted by the model, most likely due to the effect of surface sediments on the first two crustal legs of travel, which have not been corrected for. A positive phase can aslo be seen $\sim 1 \mathrm{~s}$ after the Moho arrival. This phase corresponds to the positive phase paralleling the Moho arrival in the stacked section (figure 2.34). Delay times for this phase decrease with increasing ray parameter supporting the view that this phase is a mid-crustal multiple.

Figure 2.36b shows the receiver functions for stations south of the TRL stacked in ray parameter bins as for 2.36a. Receiver functions for station RAE have been excluded, since this stations is very close to the change in crustal thickness observed in the CCP stacks. A positive phase arrives just before $5 \mathrm{~s}$. This phase corresponds to a model depth of $35 \mathrm{~km}$. Some raypaths from these stations sample the mantle converter at 65 $\mathrm{km}$ depth and positive phases can be seen on the p-stacks where this phase is expected to arrive. There is no Moho multiple observed for these stations. The predicted arrival for the Moho arrival show is close to the maximum time window for these receiver functions without accounting for the additional 2 sediment legs of travel. A positive phase is also observed at delay times of $\sim 6 \mathrm{~s}$. This phase corresponds to the positive phase seen in the stacked section just below the Moho (figure 2.34). Delay times for this phase decrease with increasing ray parameter indicating that it is likely to be a midcrustal multiple. 

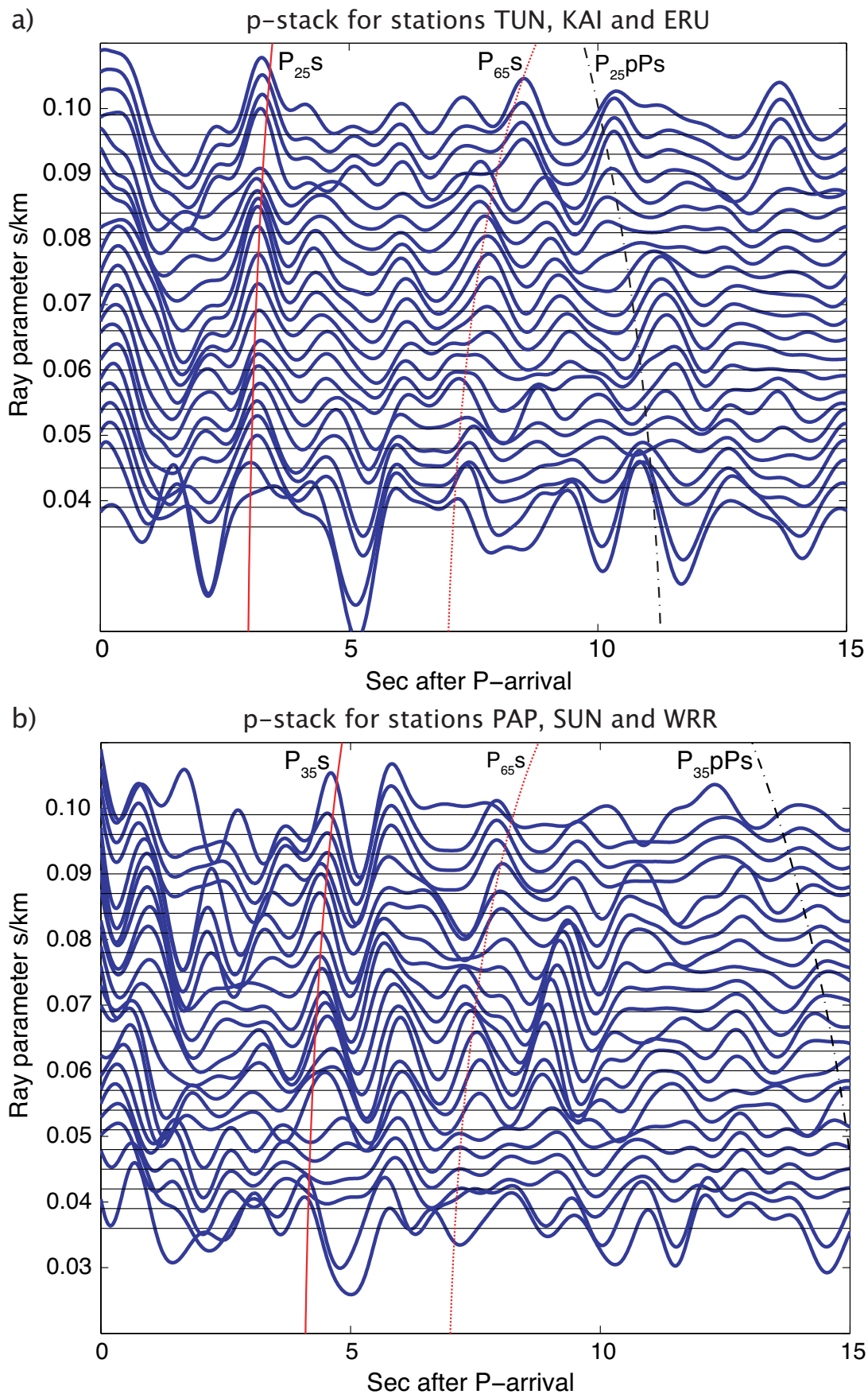

Figure 2.36: Ray parameter stacks for TRAP sites. (a) Stacks for northern stations (TUN, KAI and ERU). Ps phase moveout curves for the NZST model are shown for depths of $25 \mathrm{~km}$ (solid red line labeled $\mathrm{P}_{25} \mathrm{~s}$ ) and $65 \mathrm{~km}$ (dotted red line labeled $\mathrm{P}_{65} \mathrm{~s}$ ). The PpPs phase moveout curve for a depth of $25 \mathrm{~km}$ is also shown (black dashed line labeled $\mathrm{P}_{25} \mathrm{pPs}$ ) (b) Stacks for southern stations (PAP, SUN and WRR). Ps phase moveout curves for the NZST model are shown for depths of $35 \mathrm{~km}$ (solid red line labeled $\mathrm{P}_{35} \mathrm{~s}$ ) and $65 \mathrm{~km}$ (dotted red line labeled $\mathrm{P}_{65} \mathrm{~s}$ ). The PpPs phase moveout curve for a depth of $35 \mathrm{~km}$ is also shown (black dashed line labeled $\mathrm{P}_{35} \mathrm{pPs}$ ) 
Table 2.2: New Zealand velocity models [Maunder 2002]

\begin{tabular}{|c|c|c|c|}
\hline Model & layer depth $(\mathrm{km})$ & $V_{p}(\mathrm{~km} / \mathrm{s})$ & $V_{s}(\mathrm{~km} / \mathrm{s})$ \\
\hline \multirow{3}{*}{$\begin{array}{l}\text { New Zealand } \\
\text { Standard }\end{array}$} & $0.0-12.0$ & 5.50 & 3.30 \\
\hline & $12.0-33.0$ & 6.50 & 3.70 \\
\hline & $>33.0$ & 8.10 & 4.60 \\
\hline \multirow[t]{7}{*}{ Wellington } & $0.0-0.4$ & 4.40 & 2.54 \\
\hline & $0.4-5.0$ & 5.63 & 3.16 \\
\hline & $5.0-15.0$ & 5.77 & 3.46 \\
\hline & $15.0-25.0$ & 6.39 & 3.50 \\
\hline & $25.0-35.0$ & 6.79 & 3.92 \\
\hline & $35.0-45.0$ & 8.07 & 4.80 \\
\hline & $>45.0$ & 8.77 & 4.86 \\
\hline \multirow[t]{7}{*}{ Taupo } & $0.0-2.0$ & 3.00 & 1.70 \\
\hline & $2.0-5.0$ & 5.30 & 3.00 \\
\hline & $5.0-15.0$ & 6.00 & 3.50 \\
\hline & $15.0-33.0$ & 7.40 & 4.30 \\
\hline & $33.0-65.0$ & 7.78 & 4.39 \\
\hline & $65.0-96.4$ & 7.94 & 4.51 \\
\hline & $>96.4$ & 8.77 & 4.86 \\
\hline \multirow{5}{*}{$\begin{array}{l}\text { Stratford and Stern [2006] } \\
\text { Western North Island }\end{array}$} & $0.0-2.0$ & 4.00 & \\
\hline & $2-7$ & 5.50 & \\
\hline & $7.0-15.0$ & 6.00 & \\
\hline & $15.0-25.0$ & 6.20 & \\
\hline & $>25.0$ & 7.80 & \\
\hline
\end{tabular}




\subsubsection{Dipping layers and anisotropy}

Receiver function analysis in the previous sections uses the assumption of flat layered isotropic velocity models. Despite this, results of CCP stacking indicate the presence of a step in the Moho between stations ERU and RAE along with a southward dipping Moho south of station RAE (figure 2.34). Additionally, transverse receiver functions have a significant amount of energy on them. This indicates there is a component of out of plane converted energy from dipping layers or anisotropy. Dipping layers and anisotropy also introduce an azimuthal dependence to receiver functions [Jones and Phinney 1998, Savage 1998].

Although there is insufficient azimuthal coverage to be able to discriminate decisively between different models of dipping layers and anisotropy, models can be tested against receiver functions results north of the TRL using forward models. The method of Frederiksen and Bostock [2000] has been used to create synthetic seismograms, which can then be run through the same processing as the real data.

Constraints on the anisotropy are obtained from local earthquake and SKS splitting studies. Using local earthquakes, Morely et al. [2006] and Audoine et al. [2004] show that for the northwestern North Island there is a north-south trend in fast orientation of anisotropy. Based on no depth dependence being observed in the data, Morely et al. [2006] confine this anisotropy to depths less that $115 \mathrm{~km}$ and give a minimum anisotropy of $1.4 \%$. SKS splitting results show no anisotropy [Greve and Savage 2007], but would not be able to resolve the small delay times seen in the local data. The CCP stacking results indicate that the Moho and the boundary at $65 \mathrm{~km}$ are both flat. Layer dips have therefore been restricted to less that $5^{\circ}$. The sediments generally have a shallow southward dip $\left(<5^{\circ}\right)$ but dips for this layer may vary significantly locally. Results from the previous sections have been used to define a 3 layered model (sediment, crust and one mantle layer above a halfspace) to test the effects of introducing dipping layers and anisotropy. Receiver functions from the synthetic data were then compared to receiver functions for station KAI.

\section{Dipping layers}

Dipping layers will cause energy to be transfered to the transverse component for the dipping layer and all layers above. Any dip on the sediment layer has therefore been ignored as this will have no effect on conversions for boundaries below it and dips may vary locally below a station. A dip on the Moho produces phases of opposite polarity on the transverse receiver functions with the same timing as the Moho conversion and multiple. This is not observed at station KAI; instead the Moho conversion and multiple both have a positive polarity on the transverse receiver functions.

A small dip with a strike of $300^{\circ}$ on the $65 \mathrm{~km}$ boundary introduces a negative phase 
on the transverse receiver functions, with the same timing as the mantle arrival, for back-azimuths between $275^{\circ}$ and $345^{\circ}$ and a posistive phase between $65^{\circ}$ and $210^{\circ}$. This is similar to what is observed in the data.

\section{Anisotropy}

Anisotropy with a N-S fast orientation in the mantle layer produces a positive arrival on the transverse component with the same timing as the mantle conversion. An E-W fast direction in this layer is required to obtain the negative on the transverse observed in the data. Anisotropy in the halfspace does provide the negative on the transverse for a N-S fast orientation. However, this would place all the anisotropy below $65 \mathrm{~km}$, which would be expected to have been observed as a depth dependence in the local earthquake studies, which it was not [Audoine et al. 2004]. Crustal anisotropy with a N-S fast orientation causes a paired negative and positive phase on the transverse receiver functions. The positive phases between $275^{\circ}$ and $345^{\circ}$ have the same timing as the Moho and multiple phase on the radial receiver functions. This fits well with the observations.

While the above is only a qualitative analysis, it suggests that anisotropy north of the TRL is confined to the crust and that there may be a small NW dip on the mantle boundary. Figure 2.37 shows the receiver function results for station KAI compared with the synthetic results for a model with $3 \%$ anisotropy in the crust (N-S fast direction) and a $5^{\circ}$ dip with a strike of $300^{\circ}$ on the mantle boundary (figure 2.38). Arrival times for the major phases are matched on the radial component of the observed and the synthetic receiver functions and general trends in amplitudes are matched, but absolute amplitudes are different. This is likely to be due to the presence of noise in the real data and may also be contributed to by the sediment layer. There is also a good match between the synthetics and the data for the transverse components.

\subsection{Receiver function summary}

\section{Moho}

For the western North Island, receiver function analysis reveals a flat shallow Moho at $25 \pm 1.5 \mathrm{~km}$ depth north of the TRL. At the TRL there is a $\sim 7 \mathrm{~km}$ step in the Moho. This step in crustal thickness happens over a horizontal distance of less than $20 \mathrm{~km}$. South of the TRL the Moho is at a depth $>32 \mathrm{~km}$ and dips $\sim 5$ degrees to the south. The $V_{p} / V_{s}$ ratio for crust sampled by the TRAP array is $1.73 \pm 0.1$. This is consistent with global averages of $V_{p} / V_{s}$ for orogenic belts. Energy on the transverse receiver functions indicate the presence of crustal anisotropy with a N-S fast orientation. 

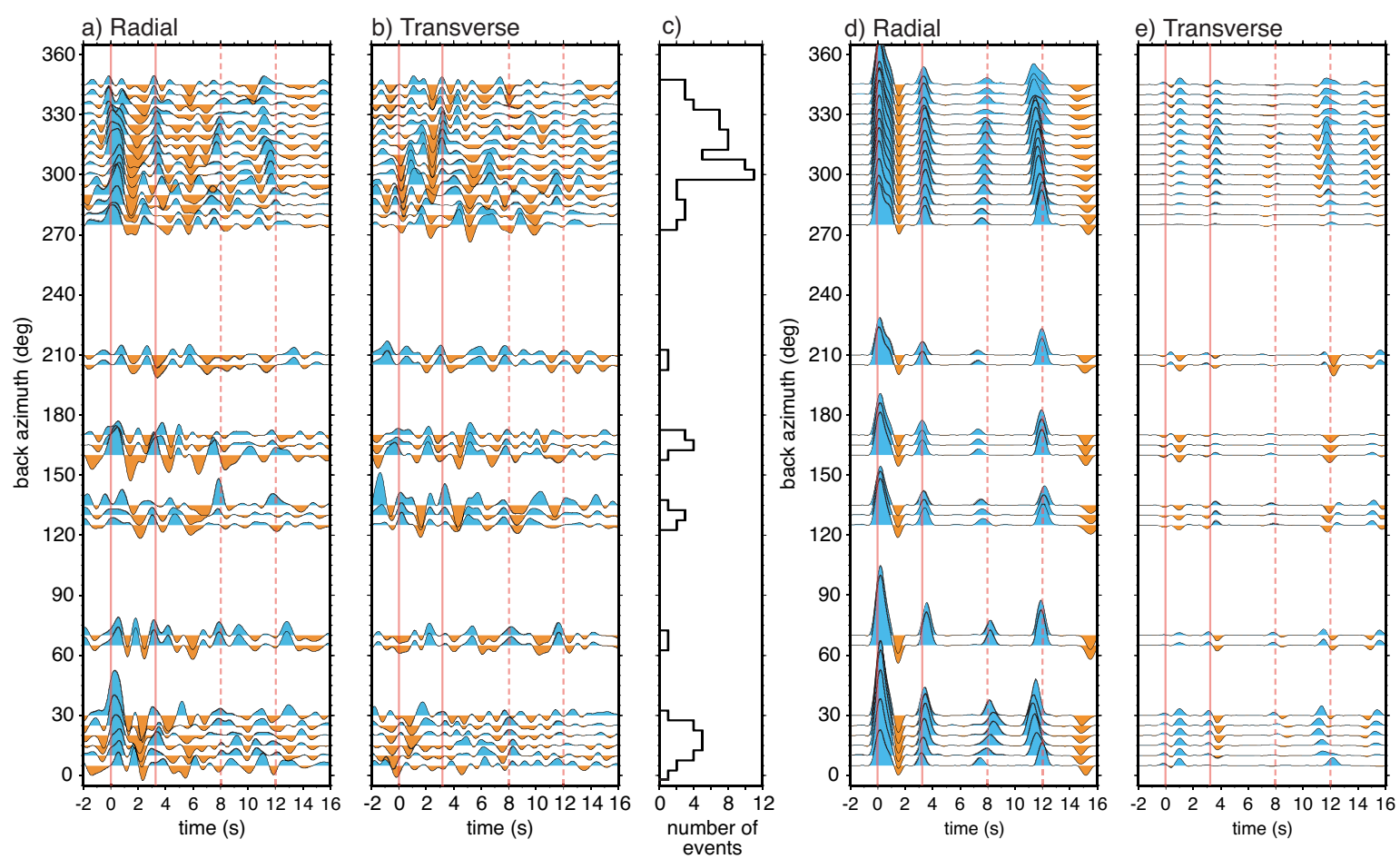

Figure 2.37: Synthetic receiver functions. (a) and (b) Shows the $\mathrm{R}$ and $\mathrm{T}$ receiver functions for stations KAI stacked in back-azimuth bins. (c) Shows the number of events in each bin. (d) and (e) show the $\mathrm{R}$ and $\mathrm{T}$ receiver functions for the synthetic data created using the model in figure 2.38. Solid red lines indicate the $0 \mathrm{~s}$ and $3.3 \mathrm{~s}$ delay times. Dashed red lines are at $8 \mathrm{~s}$ and $12 \mathrm{~s}$.

\section{Mantle boundary}

A mantle boundary is also identified in the receiver functions. This boundary is at $65 \pm 5 \mathrm{~km}$ depth and is truncated by the presence of the slab at the southern end of the line. This boundary is identified as a positive phase on the receiver functions, indicating that the boundary has higher velocities below and lower velocities above. Transverse receiver functions indicate that this layer has a small dip to the NW. The depth of this boundary coincides with an increase in $V_{p} / V_{s}$ ratio seen in tomographic studies of the region [Reyners et al. 2006]. A similar mantle boundary is observed at $75-100 \mathrm{~km}$ depth in the mantle wedge below Tonga and has been associated with compositional zonation caused by fluids rising through the wedge [Zheng et al. 2007]. 


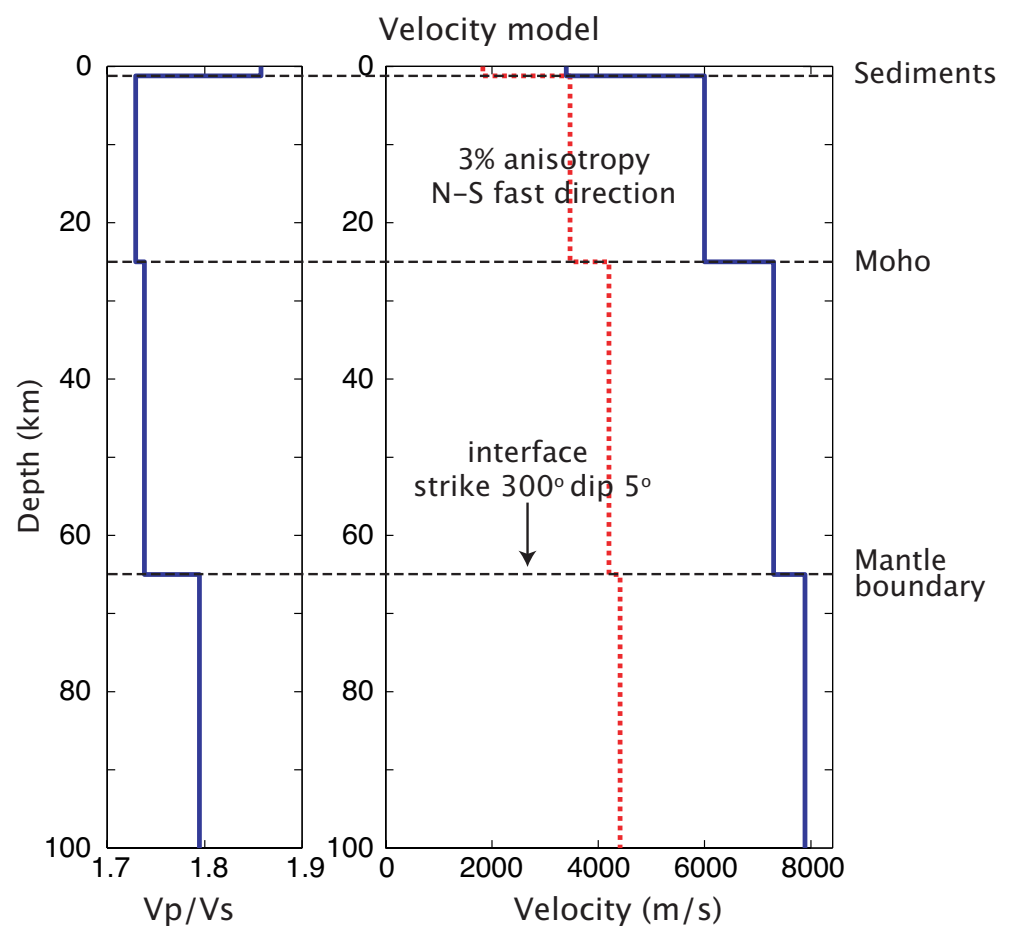

Figure 2.38: Western North Island velocity model for the area north of the TRL from receiver functions. $V_{p} / V_{s}$ ratios are shown on the left and velocities on the right. The solid blue line on the velocity plot is for $V_{p}$ and the dotted red line is $V_{s}$. Dashed lines indicate layer boundaries. Velocities in the mantle are poorly constrained but do require an increasing velocity with depth for both $V_{p}$ and $V_{s}$. 


\section{Chapter 3}

\section{SEISMIC ATTENUATION}

\subsection{Attenuation abstract}

Spectral decay analysis of $\mathrm{P}$-wave data, assuming $\alpha=0$, reveal four distinct attenuation regions below North Island New Zealand; a region of extremely high attenuation below the Central Volcanic Region $\left(1000 / Q_{p}^{\prime}=5.0\right)$, a region of intermediate attenuation below the northwestern North Island $\left(1000 / Q_{p}^{\prime}=1.9\right)$, a region of low attenuation below the southwestern North Island $\left(1000 / Q_{p}^{\prime} \leqslant 1\right)$ and a region with patches of high attenuation along the east coast fore-arc $\left(1000 / Q_{p}^{\prime} \geqslant 2\right)$. The Taranaki-Ruapehu Line (TRL) marks the boundary between the two western regions with low attenuation south of the line and intermediate attenuation north of the line.

Seismic attenuation can be used as a proxy for mantle temperature. High attenuation observed below the CVR is consistent with a hydrated mantle wedge at temperatures just above the solidus. At $30 \mathrm{~km}$ depth this equates to a temperature of $\sim 990^{\circ} \mathrm{C}$ and is indicative of the presence of $\sim 2 \%$ partial melt. The region of intermediate attenuation below the northwestern North Island is consistent with a hydrated mantle at temperatures elevated to just below the solidus $\left(\sim 940^{\circ} \mathrm{C}\right.$ at $30 \mathrm{~km}$ depth). The drop in attenuation south of the TRL is consistent with a drop in mantle temperature to temperatures more consistent with normal lithospheric mantle.

\subsection{Seismic attenuation theory}

Seismic attenuation is the dissipation of energy in seismic waves as they propagate. There are two types of seismic attenuation: intrinsic and scattering. Intrinsic attenuation describes the loss of kinetic energy by anelastic processes. Scattering attenuation is the result of displacement of energy from the direct arrival into the coda and is caused by heterogeneities reflecting and refracting part of the seismic energy. This study is mainly concerned with intrinsic attenuation and unless specified, attenuation will hereafter refer to intrinsic attenuation.

The quality factor, $Q$, is used to quantify seismic attenuation. $Q$ is the inverse of 
attenuation and is defined in terms of the fractional loss of energy per cycle of oscillation [Fowler 1990, Lay and Wallace 1995].

$$
Q=-\frac{2 \pi E}{T \frac{d E}{d t}}
$$

Where $E$ is the peak energy, $T$ is the period of the seismic wave and $t$ is time. Integrating this equation gives an expression for the variation in energy with time due to attenuation.

$$
E(t)=E_{0} e^{\frac{-2 \pi t}{Q T}}
$$

Where $E_{0}$ is the energy in the wave at $t=0$. In the context of seismology it is more useful to have this equation in terms of motion rather than energy. Displacement amplitudes are proportional to the square root of the energy [Fowler 1990] giving

$$
\begin{aligned}
A(t) & =A_{0} e^{\frac{-\pi t}{Q T}} \\
& =A_{0} e^{\frac{-\pi f t}{Q}}
\end{aligned}
$$

where $A_{0}$ is the displacement amplitude at $t=0$ and $f$ is the frequency. Equation 3.2.3 describes the exponential decay of the signals amplitude, which is superimposed on the harmonic oscillation illustrated in figure 3.1. The rate of decay increases with

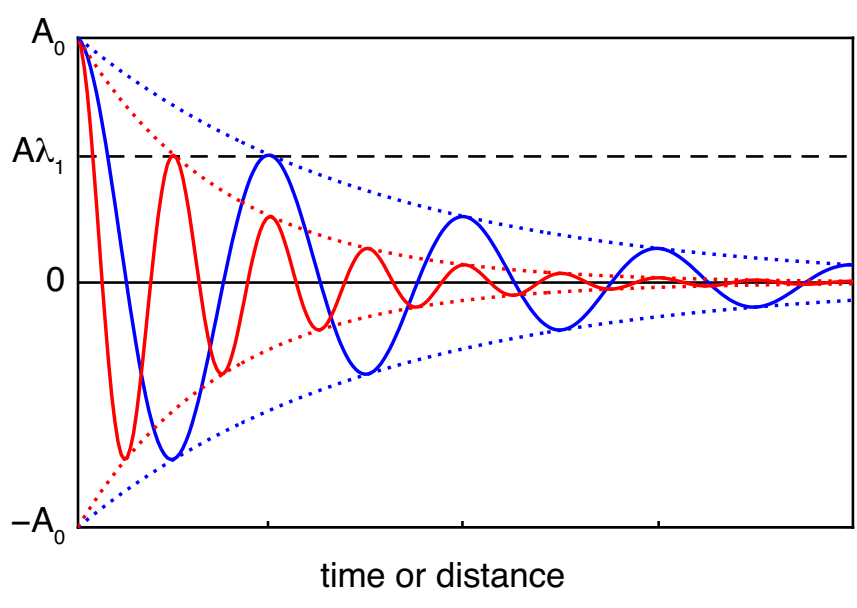

Figure 3.1: Displacement amplitude decay for a constant $Q$ value. Solid blue and red lines show the exponential decay in amplitude caused by attenuation superimposed on harmonic oscillations of frequency $f$ and $2 f$ respectively. The dotted red and blue lines show the exponential decay envelopes for the two signals given by equation 3.2.3 and the dashed black line is the amplitude after one cycle of oscillation $\left(A \lambda_{1}\right)$ for both frequencies.

decreasing $Q$ and increasing $f$. Because $Q$ is defined in terms of energy loss per cycle, an increase in frequency will result in an increase in the amount of energy that has been lost at any given time. Figure 3.1 shows the amplitude decay of two harmonic signals with frequencies $f$ and $2 f$. The amplitude of the higher frequency signal decays at twice the rate of the rate of the lower frequency, however the loss of amplitude after each cycle of oscillation is the same for both. Seismic signals encompass a wide bandwidth of frequencies, consequently the varying decay rates of different frequencies can be used 
to estimate $Q$.

\section{Attenuation mechanisms}

While the physical mechanisms that cause seismic attenuation are uncertain, some information about these processes can be obtained by looking at attenuation of different types of waves. Surface waves and free oscillations from large earthquakes have been used to determine the shear $\left(Q_{\mu}^{-1}\right)$ and compressional $\left(Q_{\kappa}^{-1}\right)$ components of seismic attenuation [e.g. Heinz et al. 1982, Widmer et al. 1991]. In the mantle $Q_{\mu}^{-1}$ is consistently higher than $Q_{\kappa}^{-1}$ indicating the mechanisms responsible for seismic attenuation are predominantly shear processes. Typically shear attenuation is at least an order of magnitude higher than compressional attenuation [Resovsky et al. 2005].

Attenuation of body waves can be related to $Q_{\mu}$ and $Q_{\kappa}$ using equations 3.2.4-3.2.6 [Lay and Wallace 1995].

$$
\begin{aligned}
Q_{s} & =Q_{\mu} \\
Q_{p}^{-1} & =\frac{L}{Q_{\mu}}+\frac{(1-L)}{Q_{\kappa}} \\
L & =\frac{4}{3}\left(\frac{v_{s}}{v_{p}}\right)^{2}
\end{aligned}
$$

Where $Q_{s}$ is the $\mathrm{S}$-wave quality factor, $Q_{p}$ is $\mathrm{P}$-wave quality factor, $v_{p}$ is the $\mathrm{P}$-wave velocity and $v_{s}$ is the S-wave velocity. For the mantle $Q_{\kappa} \gg Q_{\mu}$ [Dziewonski and Anderson 1981] and therefore, assuming a poisson solid, $Q_{p} \approx 2.25 Q_{s}$.

Globally, regions of high attenuation correlate with regions of elevated temperature. This suggests that attenuation mechanisms are thermally activated. Laboratory studies of peridotite confirm that both $\mathrm{P}$ - and S-wave attenuation have a strong temperature dependence [Jackson et al. 2004, Sato et al. 1989]. Unlike seismic velocities, attenuation is insensitive to changes in major element chemistry [Faul and Jackson 2005, Karato 2003]. This insensitivity combined with the strong temperature dependence make attenuation a good candidate for determining mantle temperatures.

\section{Frequency and grain size dependence}

A weak frequency dependence has been reported for both laboratory and field measurements of $Q$ at upper mantle conditions. This frequency dependence is described using the parameter $\alpha$ where:

$$
Q(f)=Q_{0} f^{\alpha}
$$

$Q_{0}$ is the reference the quality factor at $1 \mathrm{~Hz}$. Recent laboratory results for melt free olivine give an $\alpha$ value of 0.27 over a frequency range of $0.001-1 \mathrm{~Hz}$ [Faul and 
Jackson 2005]. A weak frequency dependence of $Q$ in the Earth's upper mantle is also observed in body wave spectra [e.g. Flanagan and Wiens 1998, Shito et al. 2004]. Although the earthquake spectra used do not provide the fine control achievable in the laboratory environment, $\alpha$ values of $0.2-0.4$ for upper mantle broadly agree with laboratory measurements.

In the presence of melt Jackson et al. [2004] show frequency dependence is enhanced over some frequency ranges due to a dissipation peak superimposed on the melt free relationship (figure 3.2). This peak moves to higher frequencies as temperature increases. For frequencies greater than that of the dissipation peak, the frequency dependence of $Q$ is enhanced. For frequencies less than that of the dissipation peak, the frequency dependence of $Q$ is diminished.

Frequency dependence often cannot be resolved from earthquake data. Attenuation estimates from body waves therefore commonly assume that $Q$ has no frequency dependence $(\alpha=0)$ [e.g. Schurr et al. 2006, Takanami et al. 2000]. This will lead to high estimates of $Q$ for $f>1 \mathrm{~Hz}$ and low estimates of $Q$ for $f<1 \mathrm{~Hz}$.

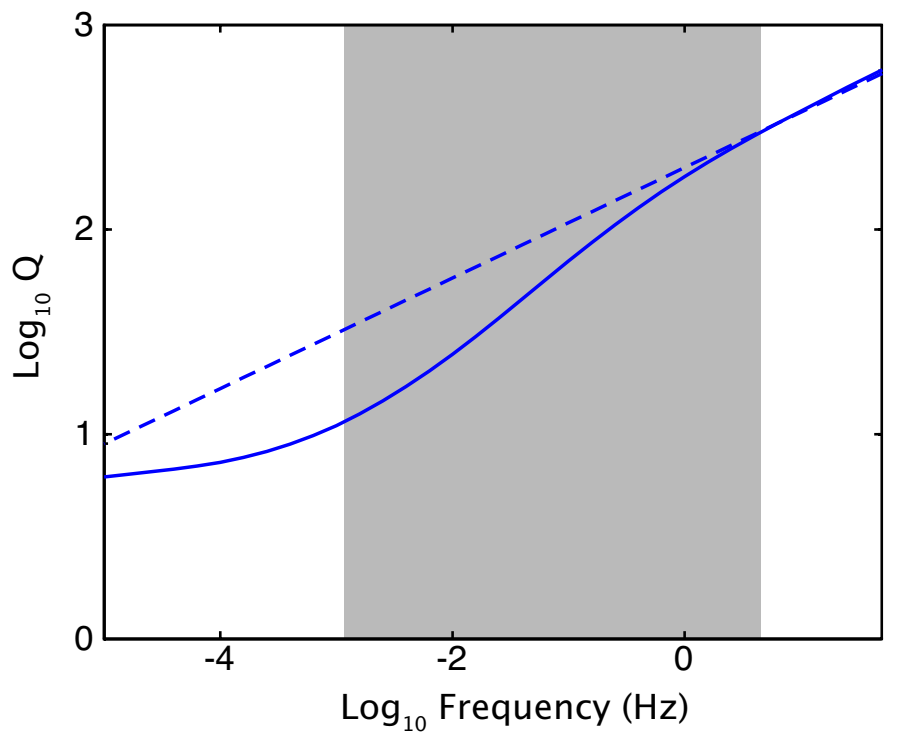

Figure 3.2: Dissipation peak model for melt-bearing olivine from Jackson et al. [2004]. The solid blue line shows the dissipation peak for olivine with a $1 \%$ melt content and a $3 \mathrm{~mm}$ grain size at $1100^{\circ} \mathrm{C}$. Note that dissipation increases with decreasing $Q$ so the peak is inverted. The dashed line shows the expected variation in quality factor with frequency for $\alpha=0.27$. The shaded region represents the frequency range where frequency dependence of $Q$ is enhanced.

Laboratory measurements of attenuation are generally carried out on fine-grained synthetic polycrystals with grain sizes of the order of $\mu \mathrm{m}$. For polycrystalline olivine $Q$ increases with increasing grain size [Jackson et al. 2004]. Mantle grain sizes are estimated to be in the range of 1-10 mm [Ave Lallemant et al. 1980]. This difference in grain size must be accounted for when comparing laboratory measurements with measurements from earthquake data. 


\section{Typical $Q$ values}

Typical mantle $Q$ values vary widely from study to study. This variation is due to the diversity of data that are used. As already discussed $Q_{p}$ is generally larger than $Q_{s}$ by a factor of $\sim 2.25$. The frequency dependence of $Q$ means that estimated $Q$ values will vary depending on the frequency range of the data used and whether this frequency dependence has been accounted for.

Global models of $Q$ generally show a decrease in $Q_{\mu}$ at the lithosphere-asthenosphere boundary. The Preliminary Reference Earth Model (PREM) [Dziewonski and Anderson 1981] reports values of $Q_{\mu}$ and $Q_{\kappa}$, at a reference frequency of $1 \mathrm{~Hz}$, for the mantle lithosphere (24-80 km depth range) of 600 and 57823 respectively. At the lithosphere asthenosphere boundary $Q_{\mu}$ drops to 80 and $Q_{\kappa}$ remains constant. More recently the QL6 model of seismic attenuation [Durek and Ekstrom 1996] reports lithospheric values of $Q_{\mu}$ and $Q_{\kappa}$ as 191 and 943 respectively. Although these values are smaller than those of the PREM, frequencies used are $\leqslant 0.01 \mathrm{~Hz}$. Converting $Q$ for a frequency of $0.01 \mathrm{~Hz}$ to the reference frequency of $1 \mathrm{~Hz}$ assuming $\alpha=0.27$ returns $Q_{\mu}=662$ and $Q_{\kappa}=3270$ for the lithosphere. The $Q_{\mu}$ values for both models are therefore consistent when frequency dependence is taken into account. Frequency dependence does not account for the differences in $Q_{\kappa}$. $Q_{\kappa}$ is usually poorly constrained compared with $Q_{\mu}$ [Resovsky et al. 2005] but, because it is usually an order of magnitude larger than $Q_{\mu}$, this parameter has less influence on the attenuation of body waves. Using equations 3.2.4-3.2.6 $Q_{s} \approx 600$ and $Q_{p} \approx 1300$ for lithospheric mantle. The QL6 model gives $Q_{\mu}=70$ and $Q_{\kappa}=943$ for the asthenosphere. $Q_{\mu}$ for this model is in agreement with the PREM value despite the frequency discrepancy. At temperatures expected in the asthenosphere the frequency dependence of $Q$ is expected to be close to 0 for the frequency range $0.01-1 \mathrm{~Hz}$ where melt is present [Faul et al. 2004]. From equations 3.2.4-3.2.6 $Q_{s}$ and $Q_{p}$ values for the asthenosphere are $\sim 70$ (from QL6) and $\sim 180$ (from both PREM and QL6) respectively.

Departures from globally averaged $Q$ models correlate with tectonic features. Low $Q$ is associated with oceanic ridges and back-arcs, while high $Q$ is associated with continental cratons [Romanowicz 1995].

\subsection{Data collection and analysis}

Local-regional earthquake data from 16 seismic stations (figure 3.3) were used for spectral decay analysis; 7 stations of the Taranaki-Ruapehu Attenuation Project (TRAP) and 9 stations from the western end of the Central North Island Passive Seismic Experiment (CNIPSE). TRAP stations were deployed in a north-south line crossing the Taranaki-Ruapehu Line (TRL) inferred from gravity, seismicity and deformation style. The CNIPSE stations used are located at the western boundary of the Central Volcanic 
Region.

TRAP stations were all short period seismographs using $1 \mathrm{~Hz}$ three-component L4C3D seismometers and Reftek 72A-07 and Reftek 130 recording systems. These instruments recorded continuously a over a 9 month period. The CNIPSE stations consisted of six short-period seismographs and three broadband seismographs. The short period instruments were portable EARSS (Equipment for the Automatic Recording of Seismic Signals) digital seismographs [Gledhill et al. 1991] with $1 \mathrm{~Hz}$ three-component L4C-3D seismometers. These seismographs were deployed for a 6 month period and recorded only when triggered. The broadband instruments comprised of Orion digital seismograph recording units and three component broadband Guralp 40T seismometers. These stations were deployed for a 12 month period and recorded continuously. Sampling rates for all instruments were set to $100 \mathrm{~Hz}$.

\subsubsection{Earthquake event selection}

For the TRAP array the GEONET catalogue [GNS Science and the Earthquake Commission 2003] was used to extract data from earthquakes with $M_{L} \geqslant 3.0$ and epicentres between latitudes 41.5-37S and longitudes 173-178E. Over the 9 month period of this deployment there were just over 1300 events that matched these criteria. Of these events, $\sim 900$ were recorded with sufficient quality to be used for spectral decay analysis. Figures 3.4 and 3.5 show spatial distribution of these earthquakes.

Because the short period instruments of the CNIPSE array did not provide continuous data, earthquakes for this array were selected based on the number of stations triggered. Earthquake events that triggered at least 4 of the short period stations were selected for analysis. Over the six month period of deployment 81 earthquakes matched the criteria. Few of these earthquakes were observed west of the CVR and the selection criteria were relaxed to include an additional 13 events for this area in order to increase azimuthal coverage. Magnitudes and locations of these earthquakes were extracted from the GEONET catalogue. Figure 3.6 show the spatial distribution of the 94 events used for spectral analysis.

\subsubsection{Spectral decay method}

A path averaged $Q$ value $\left(Q^{\prime}\right)$ can be estimated from the spectral decay of a seismic wave at time $t$. For a travel time, $t$, higher frequency waves will have undergone more cycles of oscillation than lower frequencies and will therefore have lost more energy (figure 3.1). The waveform of the seismogram is the convolution of the source waveform with path and instrument effects. In the frequency domain the displacement amplitude 


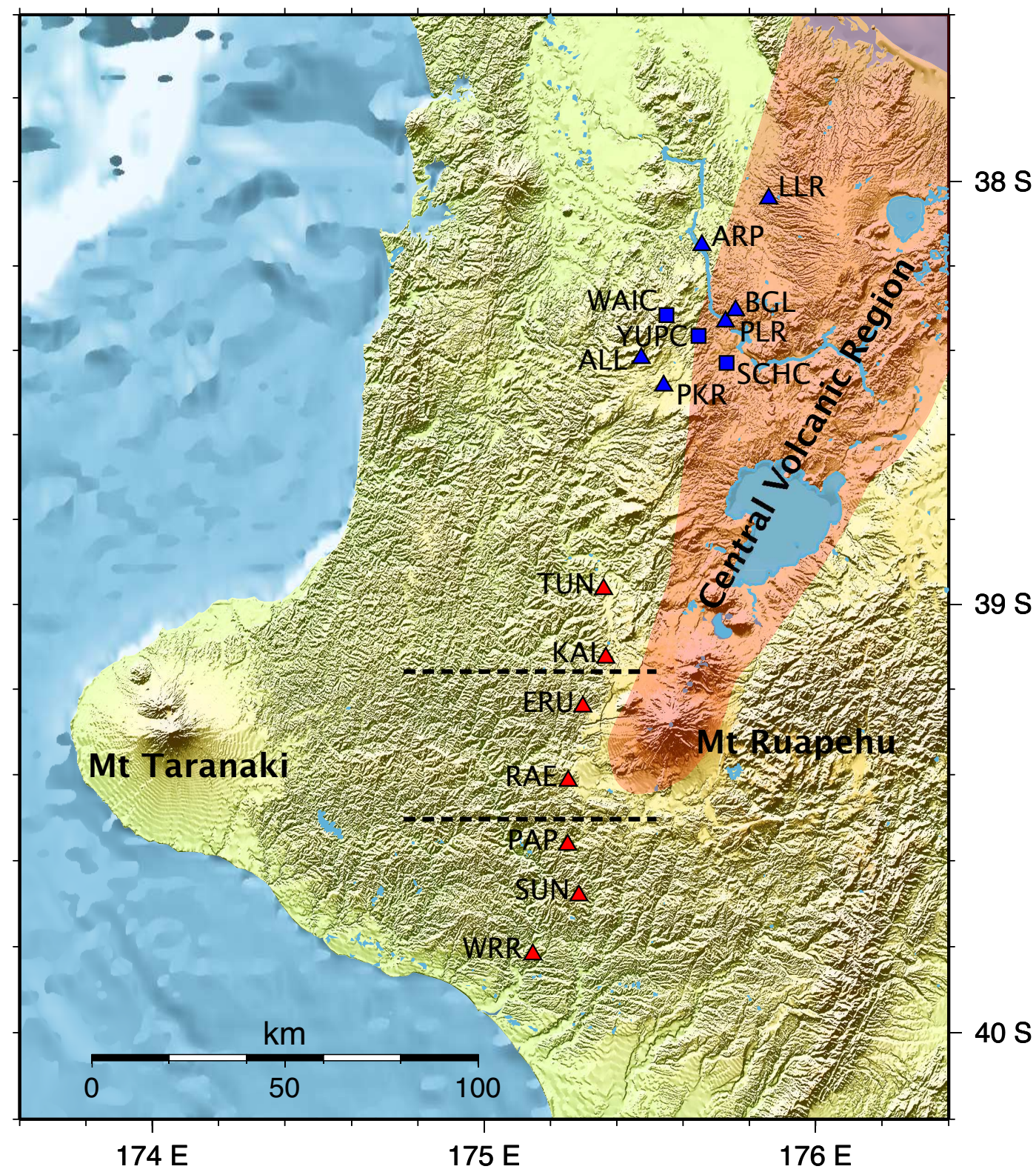

Figure 3.3: Station locations. Short period stations in the CNIPSE array are shown as blue triangles and the broadband stations are shown as blue squares. Red triangles indicate the location of the short period stations of the TRAP array. The red shaded region indicates the extent of the Central Volcanic Region. The dashed lines indicate the northern and southern limits of the proposed boundary. The northern line corresponds to northern extent of micro-seismicity at 30-40 km depth [Reyners 1978]. The southern line corresponds to the southern limit of an attenuating region seen in early national network data [Mooney 1970]. 


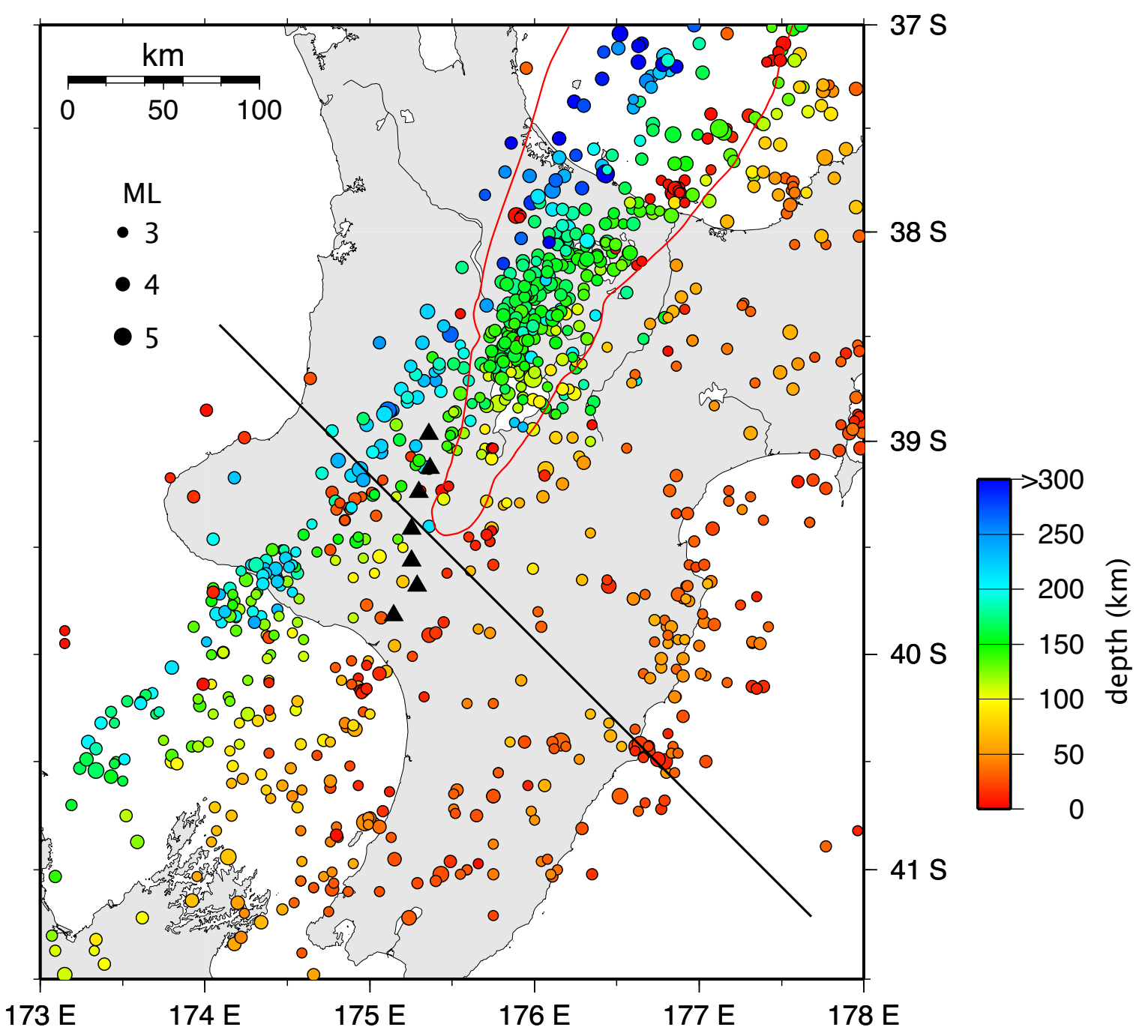

Figure 3.4: Local earthquake locations for the TRAP array. Colours indicate the focal depth and circle size indicates the magnitude of each event. TRAP stations are shown as black triangles and the boundary of the CVR is shown in red. The black line indicates the azimuth of the vertical cross section onto which earthquakes are projected in figure 3.5 


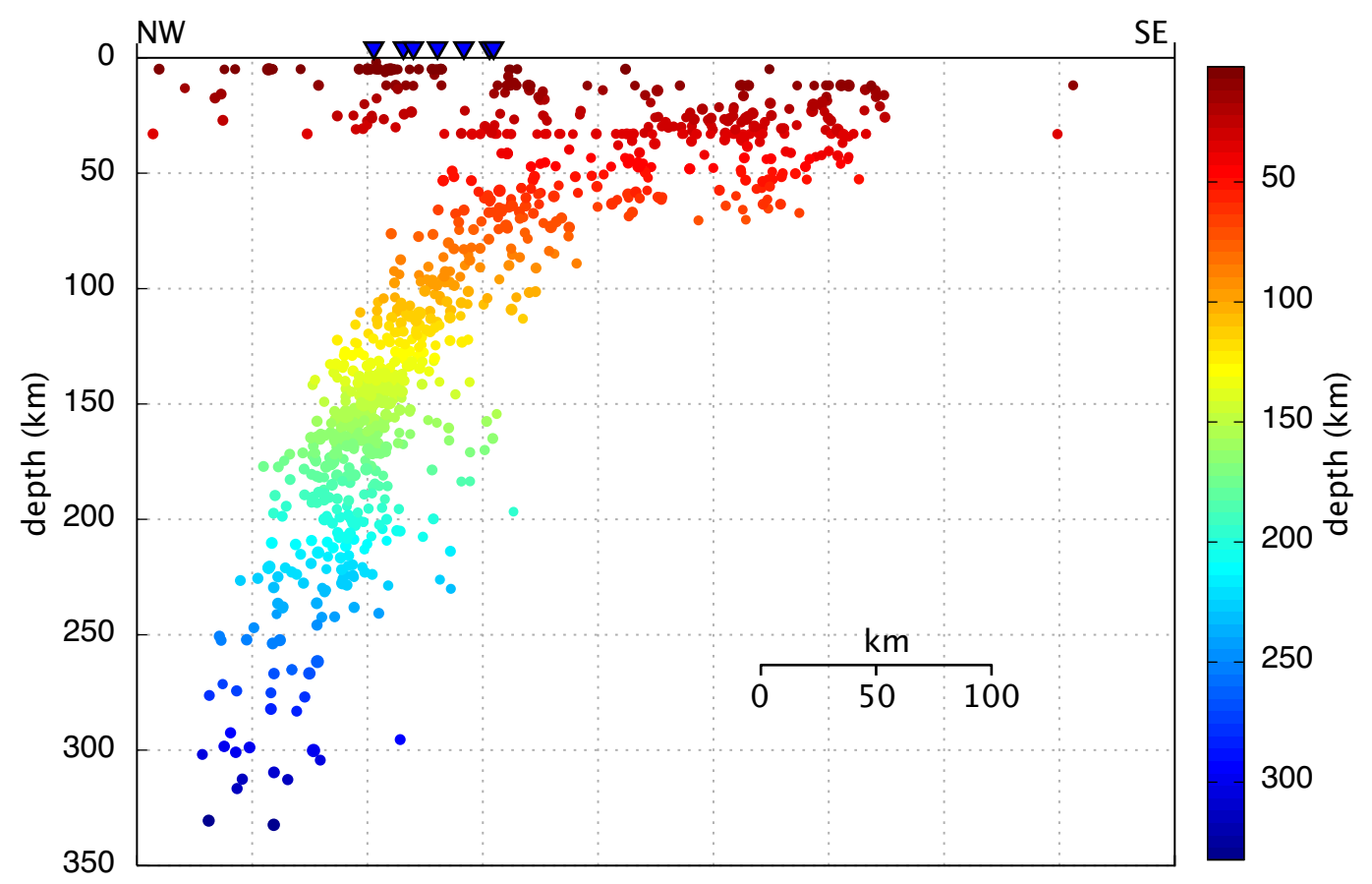

Figure 3.5: Earthquake depth profile for the TRAP array. Earthquakes are projected onto a cross section with a $135^{\circ}$ azimuth shown in figure 3.4. Colours indicate the focal depth. TRAP stations are shown as blue triangles.

spectrum $A(f)$ of a seismogram at time $t$ can be described by:

$$
A(f)=S(f) R(f) I(f) e^{-\pi f t / Q^{\prime}}
$$

where $S(f)$ combines both the source spectrum and the effects of geometric spreading, $R(f)$ is the site response spectrum and $I(f)$ is the instrument response spectrum. Collectively these terms make up $A_{0}$ in equation 3.2.3. For a $Q$ that is independent of frequency the value of $Q$ can be estimated from the frequency dependence of $A / A_{0}$ using equation 3.3.2:

$$
\frac{1}{Q^{\prime}}=-\frac{1}{\pi t} \frac{d \ln \left(A / A_{0}\right)}{d f}
$$

In order to determine a path averaged $Q$ for a station-event pair, the frequency dependent component of $A_{0}$ must first be quantified.

\section{Source spectrum}

A far-field simple source spectrum is assumed of the form:

$$
S(f)=\frac{\Omega_{0}}{1+\left(f / f_{0}\right)^{2}}
$$




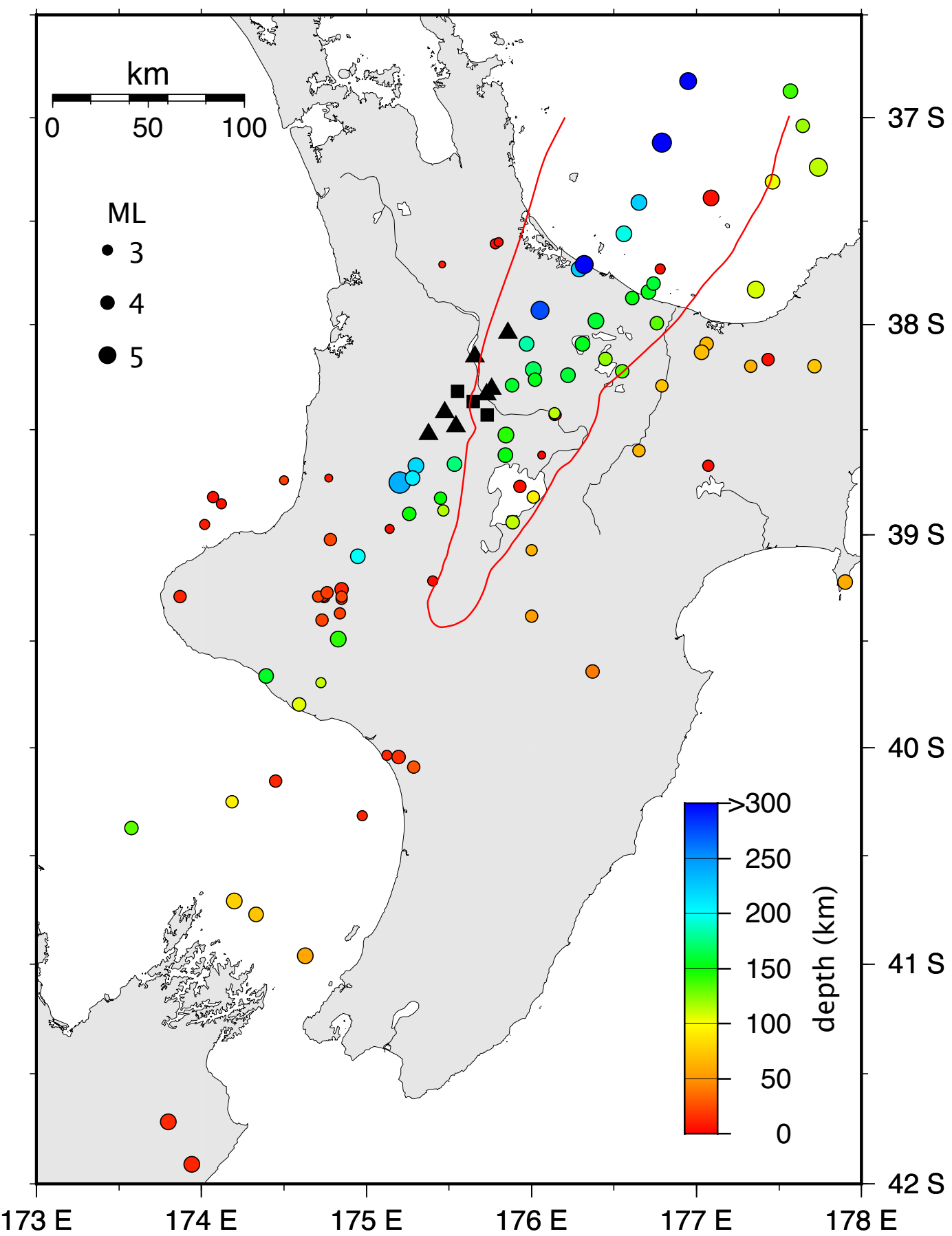

Figure 3.6: Local earthquake locations for the CNIPSE array. Colours indicate the focal depth and circle size indicates the magnitude of each event. CNIPSE shortperiod stations are shown as black triangles and broadband stations are shown as black squares. The boundary of the CVR is shown in red. 
Where $\Omega_{0}$ is the long-period amplitude and $f_{0}$ is the corner frequency given by:

$$
f_{0}=k_{p, s} v_{s}\left(\frac{16 \Delta \sigma}{7 M_{0}}\right)^{1 / 3}
$$

Where $M_{0}$ is the moment, $\Delta \sigma$ is the stress drop, $v_{s}$ is the shear wave velocity at the source and $k_{p, s}$ is a constant dependent on source mechanism [Brune 1970; 1971]. The long-period amplitude is estimated from the displacement spectra. This term takes account of the effects of geometric spreading and frequency-independent site effects. Stress drop is assumed to be constant at $3 \mathrm{MPa}$ [Abercrombie 1995, Kanamori and Anderson 1975]. The value of $k_{s}$ has been shown to be $\sim 0.33$ for a wide range of source mechanisms [Brune et al. 1979] and theoretical models show $k_{p}=1.5 k_{s}[$ Molnar et al. 1973]. The shear wave velocity at the source is estimated from the 1D regional velocity models used to locate earthquakes in New Zealand [Maunder 2002].

Moments are calculated from the local magnitudes provided by the GEONET catalogue using the following relationships for plate margin earthquakes given by Hanks and Boore [1984]:

$$
\begin{array}{rlr}
\log _{10} M_{0} & =1.0 m_{b}+11.15 & m_{b} \leqslant 4.4 \\
\log _{10} M_{0} & =2.0 m_{b}+6.75 & 4.4 \leqslant m_{b} \leqslant 6.9 \\
m_{b} & =M_{L}-0.4 &
\end{array}
$$

\section{Site response}

Site responses are assumed to be independent of frequency, in which case this term is included in the source term. Two situations where this assumption may break down are for sites close to topographic extremes and soft rock sites. Both topographic extremes and soft rocks can produce peaks in site response spectra [Boore and Joyner 1997, Chavez-Garcia et al. 1996]. The effect this has on the spectral decay should be small for smoothed spectra provided sufficient bandwidth is used [Bock and Clements 1982].

Frequency-dependent site response will remain constant at a station for all stationevent pairs. Spectral decay from seismic attenuation, however, is also a function of travel time and therefore any frequency-dependent site response unaccounted for in the spectra will be more significant for events with shorter travel times.

\section{Instrument response}

Different instrument systems have different responses and will therefore distort the incoming waveform in different ways. Figure 3.7 shows the response spectra for the instruments from the TRAP and CNIPSE arrays [Gledhill et al. 1991, IRIS PASSCAL 2005]. For the frequency range used in this study $(1-15 \mathrm{~Hz})$ only the instrument gain 
varies between instruments. At lower frequencies the differences between the systems is more marked since the corner frequency of the broadband CMG-40T is much lower than that of the L4C-3D seismometer.

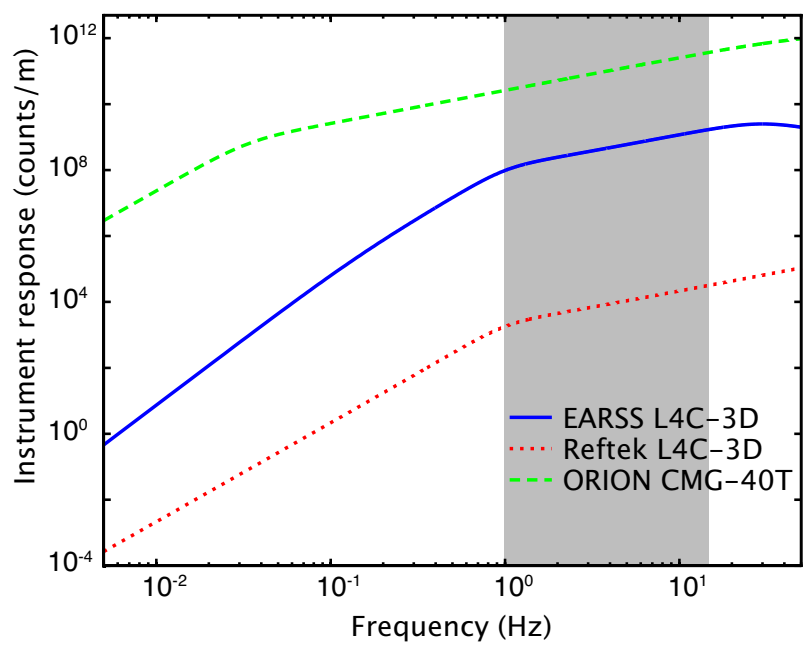

Figure 3.7: Instrument responses. Displacement responses for all station configurations used are shown. The grey shaded area indicates the frequency range used in this study. Within this frequency range the only difference between instrument responses is the gain.

To ensure instrument responses have been accounted for correctly, huddle tests were conducted. A huddle test is a short recording using instruments located close together. A distant earthquake recorded by instruments in a huddle test can be assumed to provide the same input into all instruments in the huddle test. Huddle tests allow the calibration of a group of instruments and can help identify defective hardware. After the instrument response is removed from recorded data, event waveforms and spectra for different instruments should look the same.

Data from two separate huddle tests have been used to ensure that instrument responses are correct and to check the performance of the seismometers. The first of these was conducted prior to the CNIPSE deployment. Two of the ORION CMG40T systems and five of the EARSS L4C-3D systems were tested. The second huddle test was conducted at the end of the TRAP deployment and used all the L4C-3D seismometers used in the TRL deployment along with three different DAS (Data Acquisition System) units; the EARSS, Reftek 72A-07 and Reftek 130-01. This was done so that a comparison of all the systems used throughout both the TRAP and CNIPSE deployments could be made. The reference system is the EARSS system.

In figure 3.8 vertical-component data corrected for instrument response is shown for an earthquake recorded during the first huddle test. The data has been high-pass filtered at $0.5 \mathrm{~Hz}$. Waveform and spectral data for both instruments are nearly coincident indicating that the instrument responses for the two instrument set ups has been accounted for correctly.

Figure 3.9 shows vertical-component data, corrected for instrument response, for an earthquake recorded during the second huddle test. The data has been high-pass 


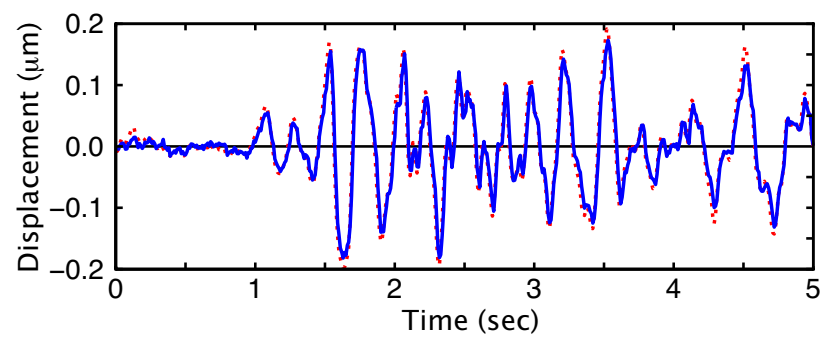

Figure 3.8: ORION-EARSS huddle test. The top figure displays the vertical-

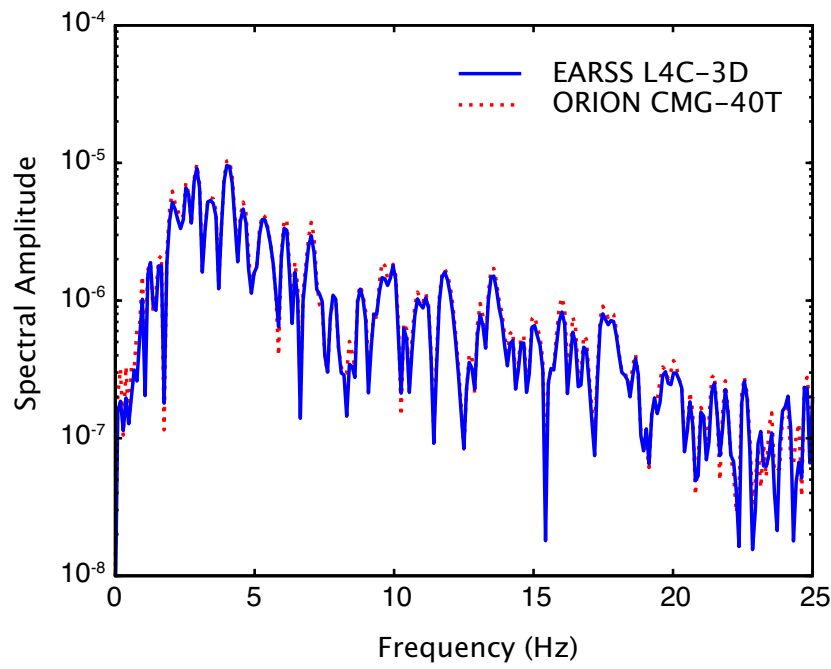
component of the $\mathrm{P}$-wave displacement for a $M_{L} 3.58$ earthquake recorded during the huddle test. The solid blue line indicates the displacement recorded on the EARSS system and the dotted red line indicates the displacement recorded by the ORION system. Both have been corrected for instrument response. The lower figure shows the spectra of the waveforms above.

filtered at $0.5 \mathrm{~Hz}$. Waveform and spectral data for both instruments are nearly coincident, indicating that the instrument responses for the three instrument set ups has been accounted for correctly.

\subsubsection{Processing}

Earthquake data for the TRAP array was cut from the continuous data using locations and origin times from the GEONET catalogue and AK135 travel time estimates [Kennett et al. 1995]. Initially $300 \mathrm{~s}$ windows were cut, including the $60 \mathrm{~s}$ preceding the expected first arrival. For the CNIPSE array triggering provided data 10-30s prior to the first arrival and window lengths varied depended on the coda decay of the event. Pre-processing of the seismograms included demeaning, tapering, instrument response removal and a high-pass filter at $0.55 \mathrm{~Hz}$. On inspection of the cut data it was discovered that for much of the data set the S-wave arrivals were attenuated to amplitudes less than those of the P-wave coda (figure 3.10). For the triggered data this sometimes meant that the S-wave data was not recorded and therefore only the $\mathrm{P}$-waves have been analysed in this study.

Five-second windows beginning $\sim 1 \mathrm{~s}$ before the onset of the P-wave, were extracted from the vertical component data for spectral analysis. Five second windows were also extracted immediately before these $\mathrm{P}$-wave windows to be used to estimate the spectral noise. An example of the noise and P-wave windows is shown in figure 3.11. These windows were tapered and then Fourier transformed into the frequency domain. 

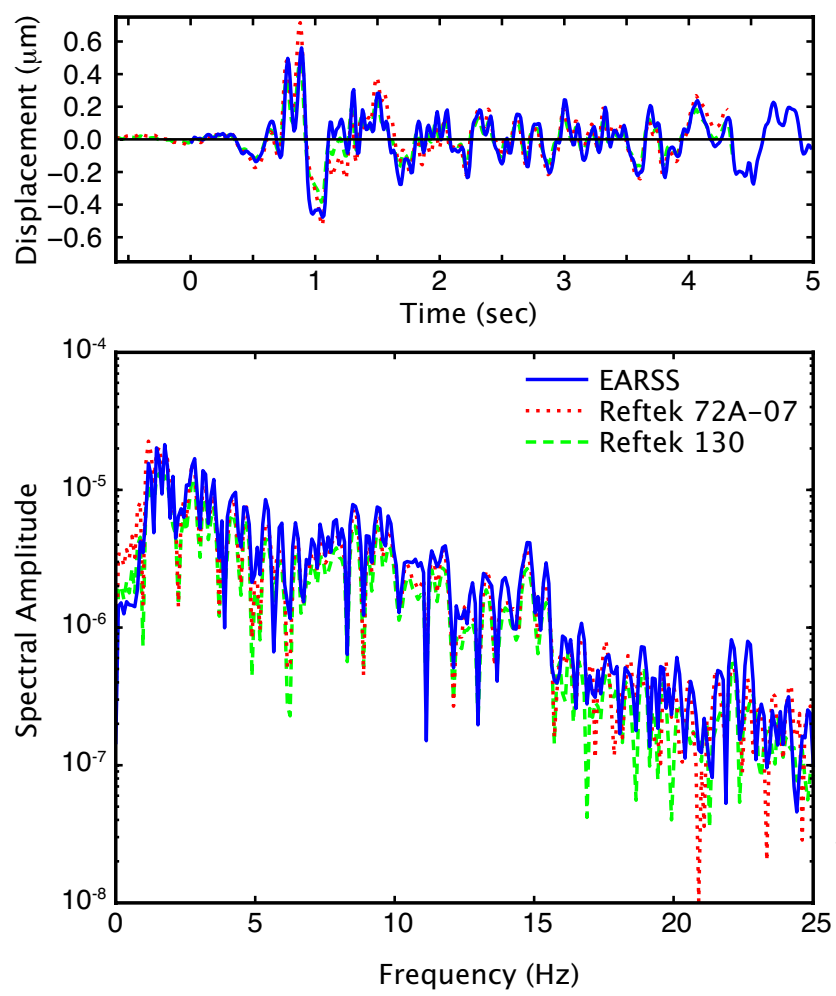

Figure 3.9: EARSS-Reftek huddle test. The top figure displays the verticalcomponent of the $\mathrm{P}$-wave displacement for a $M_{L} 5.17$ earthquake recorded during the huddle test. The solid blue line indicates the displacement recorded on the EARSS system, the dotted red line indicates the displacement recorded Reftek 72A-07 system and the dashed green line indicates the displacement recorded Reftek 130 system. All have been corrected for instrument response. The lower figure shows the spectra of the waveforms above.

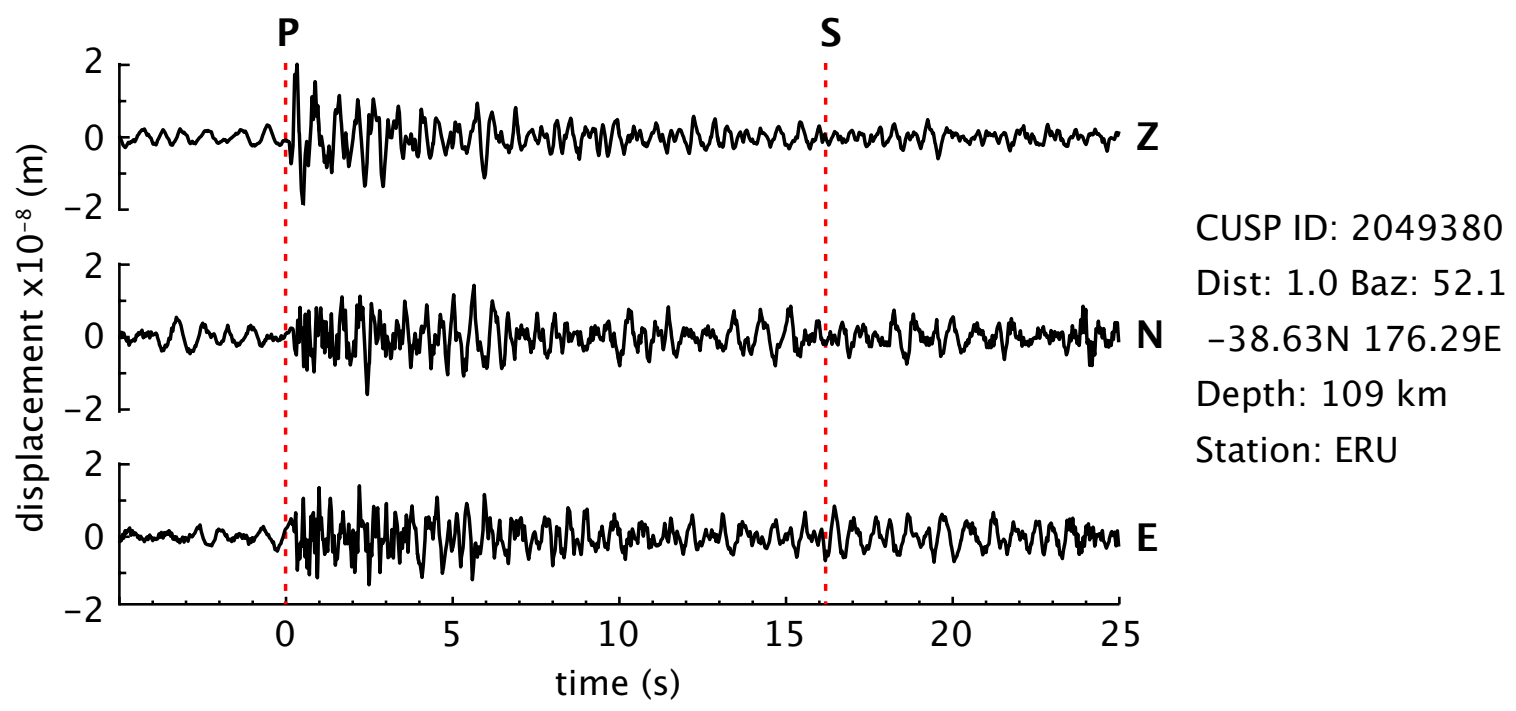

Figure 3.10: Event with attenuated S-wave recorded at station ERU. The dashed red lines indicate the expected P-and S-waves arrival from the AK135 tables. Although the $\mathrm{P}$-wave arrival is clearly observed on all three components there is no clear $\mathrm{S}$-wave arrival. 


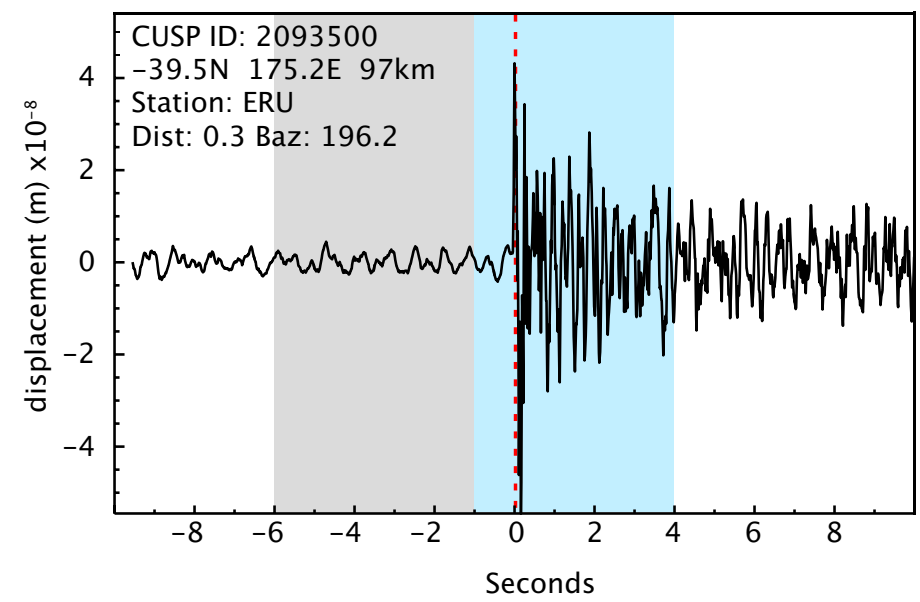

Figure 3.11: Vertical component P-wave data windows used for spectral analysis is shown in blue and the noise window is shown in grey. The dashed red line indicates the expected arrival time of the Pwave from the AK135 tables.

Once in the frequency domain, spectra were smoothed using a three point triangular smoothing function. Signal to noise ratios were calculated for use in quality control. Noise spectra were subtracted from the P-wave spectra. The resulting displacement spectra include source, site and propagation effects. The source spectra and the frequency-independent component of site response, calculated using equation 3.3.3, were then removed.

The gradient of the spectral decay $\left(d\left(\ln A / A_{0}\right) / d f\right)$ was calculated by least-squares linear regression over the frequency range 1-15 Hz. Frequencies with signal to noise ratios less than 2 were excluded from the regression. Where signal to noise ratios dropped below 2 for a bandwidth of more than $1 \mathrm{~Hz}$ the linear regression was carried out for frequencies below this frequency band.

Finally, P-wave travel times were estimated from origin times provided by the GEONET catalogue and $Q^{\prime}$ was calculated using equation 3.3.2.

The spectral decay technique used here provides good estimates of $Q^{\prime}$ where there is high attenuation; however, where attenuation is low errors may exceed the variation in attenuation. For example, typical errors in magnitude for this data set are \pm 0.2 $M_{L}$. An error in magnitude will effect the corner frequency and hence the shape of the source function. Where $M_{L}$ is underestimated the slope of the spectral decay will also be underestimated and where $M_{L}$ is overestimated the spectral decay will be overestimated. Typical errors in $M_{L}$ for this study will result in error in the spectral slope of \pm 0.02 [GNS Science and the Earthquake Commission 2003]. By comparison, the spectral slope caused by seismic attenuation for a raypath with a $20 \mathrm{~s}$ travel time and $Q^{\prime}$ values of 2000,500 and 250 will be $0.03,0.13$, and 0.25 respectively. For the low attenuation raypath $\left(Q^{\prime}=2000\right)$ the uncertainty in spectral decay caused by the magnitude is of the same order as the spectral decay.

Another potential source of error is the assumption of a constant stress drop. As with magnitudes, an error in the stress drop will effect corner frequency of the source 
function. The stress drop used for this study $(3 \mathrm{MPa})$ is a typical stress drop for intraplate earthquakes, however stress drops vary globally from 1-10 $\mathrm{MPa}$ [Kanamori and Anderson 1975]. Overestimates of stress drop will lead to and underestimate in the spectral slope and vice versa. For the worst case scenario $(\Delta \sigma=1$ or $10 \mathrm{MPa}$ and $\left.M_{L}=3\right)$ the error in the spectral slope will be \pm 0.04 .

For shorter travel times, errors in the measurement of the spectral decay slope will result in larger errors in $Q$. This and the far-field assumption require that data from events within $25 \mathrm{~km}$ of the station be excluded from the following analysis.

\subsection{Attenuation results}

Figure 3.12 shows data for three typical attenuation regimes. Signal amplitudes are generally well above the ambient noise for the frequency range 1-15 Hz. The effect of seismic attenuation on the frequency content can be clearly observed in both spectra and time domain data. High attenuation paths (left) have less high frequency content and steeper spectral decay slopes than low attenuation paths (right). For low attenuation raypaths errors in spectral decay slopes are often of the same order of magnitude as the spectral decay itself. As a result sometimes negative values of $Q_{p}^{\prime}$ are returned, which is physically impossible. It has therefore been assumed that negative values of $Q_{p}^{\prime}$ represent low attenuation raypaths. Where a positive value of $Q^{\prime}$ is outside the $95 \%$ error bounds of the measurement, the $Q_{p}^{\prime}$ value has been excluded from further analysis.

\subsubsection{CNIPSE data attenuation results}

Results for the CNIPSE data can be seen in figures 3.13 and 3.14. Data reveal two distinct attenuation regions. The first is a high attenuation region, which encompasses most of the CVR. The second region has lower attenuation and encompasses the northwestern corner of the CVR and the rest of the western North Island.

For stations either inside or on the edge of the CVR (LLR, ARP, YUPC, BGL, PLR and SCHC figure 3.3), the 133 raypaths that cross the CVR have an average $Q_{p}^{\prime}$ value of $200 \pm 20$. The consistency of measurements between stations indicates that the effect of any frequency-dependent site response is small. $Q_{p}^{\prime}$ values increase for raypaths that traverse the northwestern corner of the CVR. Raypaths from an earthquake more than $100 \mathrm{~km}$ east of the CVR also show a decrease in attenuation (circled event in figure 3.13). A large portion of these raypaths are outside the CVR indicating that pathaveraged attenuation increases with increasing travel time outside the CVR. Raypaths that do not cross the CVR have more variable $Q_{p}^{\prime}$ measurements but typically $Q_{p}^{\prime}$ is higher. Figure 3.13 illustrates the lateral variation of attenuation for the CNIPSE data. 

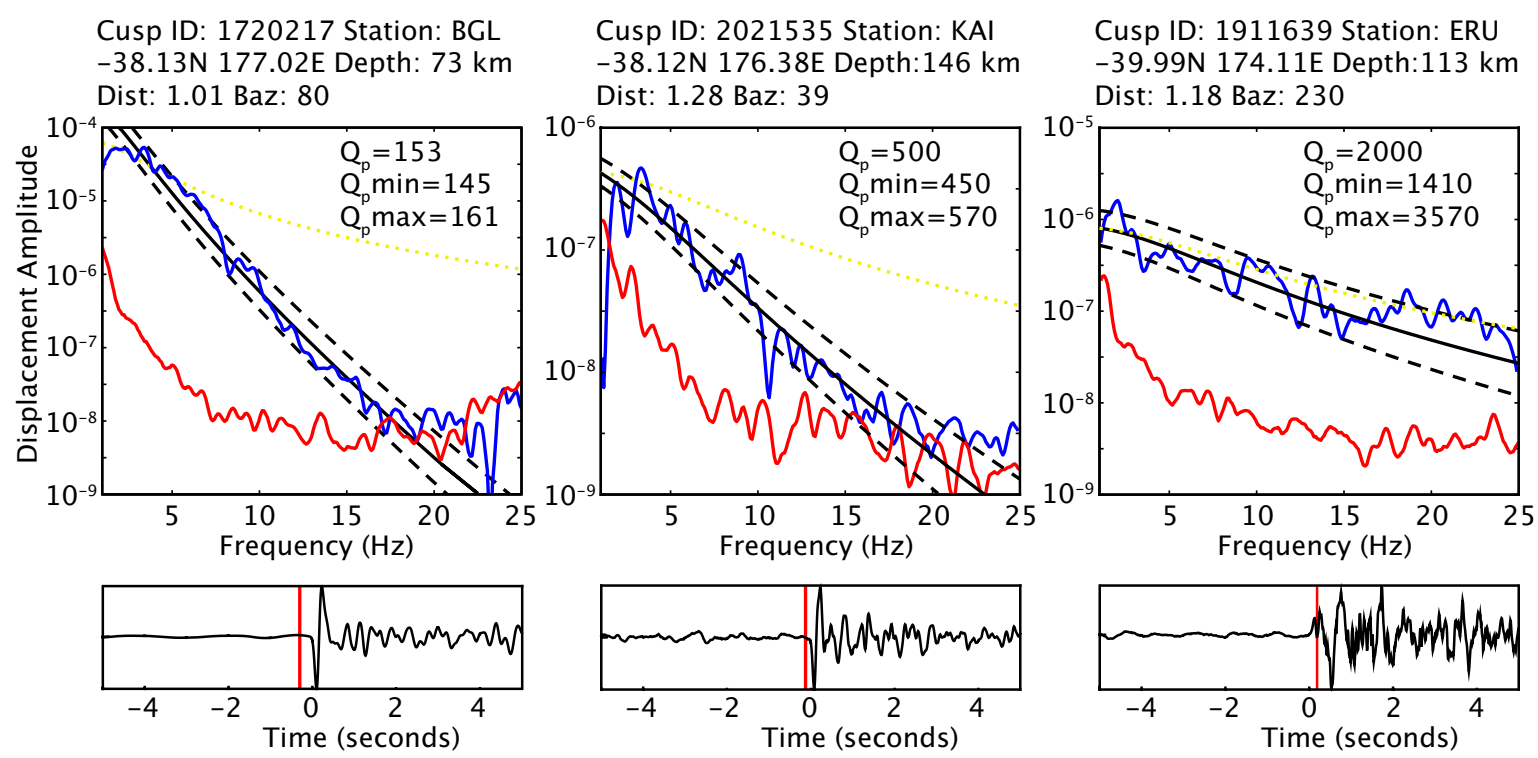

Figure 3.12: Typical attenuation data fit. Spectra (top) and time domain data (bottom) are shown for three typical attenuation paths. Note the different scales on the y-axis. The first is for a high attenuation CVR path, the second for a medium attenuation north-west North Island path and the last is for a low attenuation south-west North Island path. Smoothed spectra corrected for noise are shown in blue. Noise spectra are shown in red. Dotted yellow lines indicate the estimated source function. Solid black lines show the data fit for the best estimate of $Q_{p}^{\prime}$ and the dashed lines indicate the upper and lower limits of the $95 \%$ confidence interval. In the time domain plots below the red line marks the expected P-wave arrival time from the AK135 model.

CVR raypaths show little variation in $Q_{p}^{\prime}$ with event depth. Outside the CVR, however, $Q_{p}^{\prime}$ decreases with increasing event depth down to $\sim 150 \mathrm{~km}$ and then starts to increase again. Figure 3.14 shows the depth dependence of $Q_{p}^{\prime}$ for the two attenuation regions. There is a large scatter in $Q_{p}^{\prime}$ values for crustal earthquakes outside the CVR. This may be a result of an increase in the influence of scattering in the crust. The high $Q_{p}^{\prime}$ values for deep events is likely to be a result of low attenuation slab paths. Also shown in figure 3.14 are the variations in $Q_{p}^{\prime}$ that would be expected for three $Q$ models. The path-averaged attenuation for a layered model can be calculated using equation 3.4.1:

$$
Q_{p}^{\prime}=\frac{\sum t_{n}}{\sum t_{n} / Q_{n}}
$$

where $Q_{n}$ is the quality factor for layer $n$ and, $t_{n}$ is the percentage of the overall travel time the raypath spends in layer $n$. The first model is for a $Q_{p}$ of 200 for the crust and mantle wedge $(0-150 \mathrm{~km})$ and 4000 for the subducting slab $(>150 \mathrm{~km})$. The model provides a reasonable fit to CVR raypaths. The second model is the same as the first but a high $Q_{p}$ crustal layer $\left(Q_{p}=1200\right.$ between depths of $\left.0-25 \mathrm{~km}\right)$ has been added. For this model $Q_{p}^{\prime}$ drops sharply for events with depths greater than $25 \mathrm{~km}$ and 


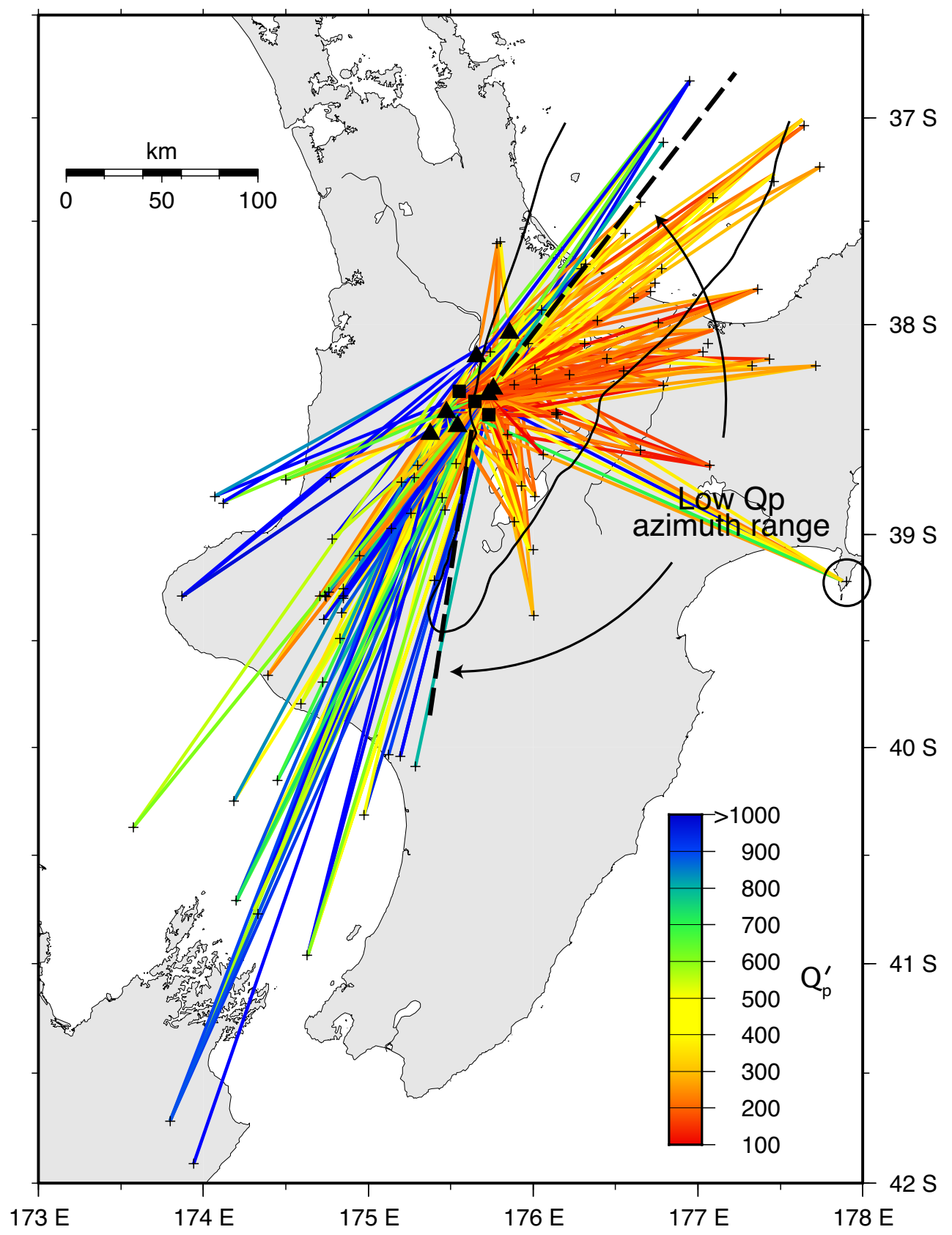

Figure 3.13: CNIPSE data $Q_{p}^{\prime}$ results. Raypaths are colour coded according to their path-averaged $Q_{p}$ values. Red indicates high attenuation and blue indicates low attenuation. All stations show the same pattern of low $Q_{p}^{\prime}$ values in an azimuth range from $\sim 40-200^{\circ}$. The dashed line shows the extent of the low $Q_{p}^{\prime}$ ' azimuth range and is approximately coincident with the eastern CVR. $Q_{p}^{\prime}$ gradually increases in the northwestern corner of the CVR. For earthquakes east of the CVR, $Q_{p}^{\prime}$ increases as the proportion of the ray path east of the CVR increases as seen by the circled evet on the east coast. Typical errors in $Q_{p}^{\prime}$ measurements are $\pm 10 \%$. 


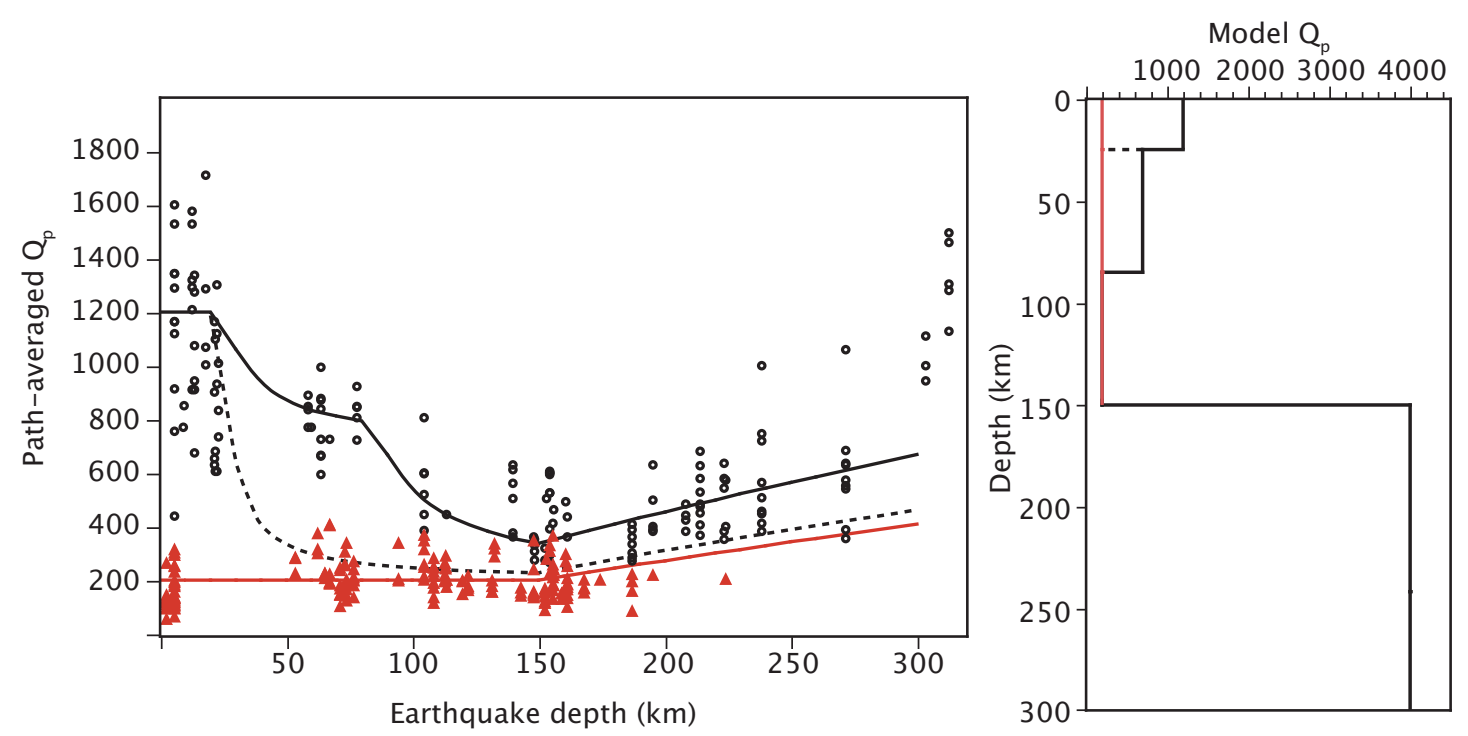

Figure 3.14: Earthquake depths vs. $Q_{p}^{\prime}$. On the left $Q_{p}^{\prime}$ values are plotted against earthquake depth. Events with raypaths within the low Qp azimuth range are shown as red triangles and raypaths outside this region are shown as black circles. Expected $Q_{p}^{\prime}$ for the three $Q_{p}$ models shown on the right are also plotted. The solid red line is for a model with constant low $Q_{p}\left(Q_{p}=200\right)$ in the crust and the full depth of the mantle wedge and a high $Q_{p}$ slab $\left(Q_{p}=4000\right)$. A high $Q_{p}$ crustal layer $\left(Q_{p}=1200\right)$ is added for the model represented by the dashed black line. An additional layer with $Q_{p}$ of 700 has been added directly below the crust for the last model and is represents by the solid black line.

illustrates that the depth dependence of the raypaths outside the CVR cannot be due solely to a high crustal $Q_{p}^{\prime}$. Changing the $Q_{p}$ value in the crust up or down in fact has little effect on the shape of the $Q_{p}^{\prime}$ vs. depth curve below $50 \mathrm{~km}$ depth. The last model has an additional intermediate attenuation layer directly below the crust $\left(Q_{p}=700\right.$ for depths $25-85 \mathrm{~km}$ ). This model fits provides a reasonable fit to the observed variations in $Q_{p}^{\prime}$ with event depth for events outside the CVR.

\subsubsection{TRAP data attenuation results}

$Q_{p}^{\prime}$ values from the TRAP array have a larger range than the CNIPSE data and it is therefore easier to describe the variations of $1000 / Q_{p}^{\prime}$. Lateral variations in $1000 / Q_{p}^{\prime}$ have been mapped for each station separately in figures 3.15-3.16. For each stationevent pair $1000 / Q_{p}^{\prime}$ values have been binned according to event epicentre. Bins are approximately $20 \times 20 \mathrm{~km}$ squares. For each bin a weighted mean $1000 / Q_{p}^{\prime}$ is calculated. Weightings are proportional to 1 /error of each value so that $1000 / Q_{p}^{\prime}$ values with large errors are down-weighted. The resulting contour plots of $1000 / Q_{p}^{\prime}$ can be split into two categories. The first is for the four northernmost stations (TUN, KAI, ERU and RAE) and the second is for the southern 3 stations (PAP, SUN and WRR). 
For the northern stations the attenuation is low $\left(1000 / Q_{p}^{\prime}<1\right)$ for events from the south west (figure 3.15). For stations KAI, ERU and RAE the 1000/ $Q_{p}^{\prime}=1$ contour runs $\mathrm{E}-\mathrm{W}$ at approximately the same latitude as the change in crustal thickness seen in the receiver functions. East of the CVR the $1000 / Q_{p}^{\prime}=1$ contour swings southward to an azimuth that approximately follows the main tectonic strike of New Zealand. Station TUN was only operational for 50 days and consequently less data was available for this station. The limited data set for this station still shows the N-S change in attenuation seen in the other three northern stations. Along the eastern side of the North Island there are isolated regions of high attenuation $\left(1000 / Q_{p}^{\prime}>2\right)$.

The southern stations do not show the defined region of low attenuation for earthquakes from the southwest (figure 3.16). For these three stations earthquakes from most azimuths have low attenuation. As with the northern stations, regions of high attenuation are observed for these stations along the east coast of the North Island. An isolated region of high attenuation on the west coast at approximately the same latitude as the change in crustal thickness can be seen in the results of four of the stations (ERU, RAE, PAP and SUN). This is the result of crustal earthquakes close to the volcanic centre of Mt Taranaki (figure 1.1).

In common to both the northern and southern stations are regions of high attenuation along the fore-arc region of the eastern North Island. In particular a region at $\sim 40.5$ $\mathrm{S}$ and a region close to Hawke Bay, can be seen in the data from all stations except TUN. Earthquakes from these two regions have $1000 / Q_{p}^{\prime} \geqslant 2$. These two regions align with a high attenuation region seen below the eastern North Island in an attenuation study of the southern North Island [Eberhart-Phillips et al. 2005].

Earthquakes north of the change in crustal thickness and west of the CVR are all from depths $<50 \mathrm{~km}$ or $>120 \mathrm{~km}$ (figure 3.4). A depth dependence of attenuation cannot therefore be determined from this data set.

Figure 3.17 shows mean values of $1000 / Q_{p}^{\prime}$ for earthquakes from the low attenuation region in the southwest and for events north of the change in crustal thickness. Mean values have been normalised using RAE as a reference station to eliminate any event dependent changes. Station RAE was chosen as a reference station since it is the closest station to the change in crustal thickness that was operational for the entire deployment. First the weighted mean $1000 / Q_{p}^{\prime}$ values were calculated for sets of common events for two station groups. Weights used in the calculation were determined based on the errors for the individual measurements in the same way as for the contour plots.

The first set of events used was from the low attenuation region in the southwest. The first station group includes stations north of the change in crustal thickness (TUN, KAI and ERU) and the reference station RAE. Excluding station TUN, this group of stations had southwest 43 events in common. Station TUN was excluded because the 

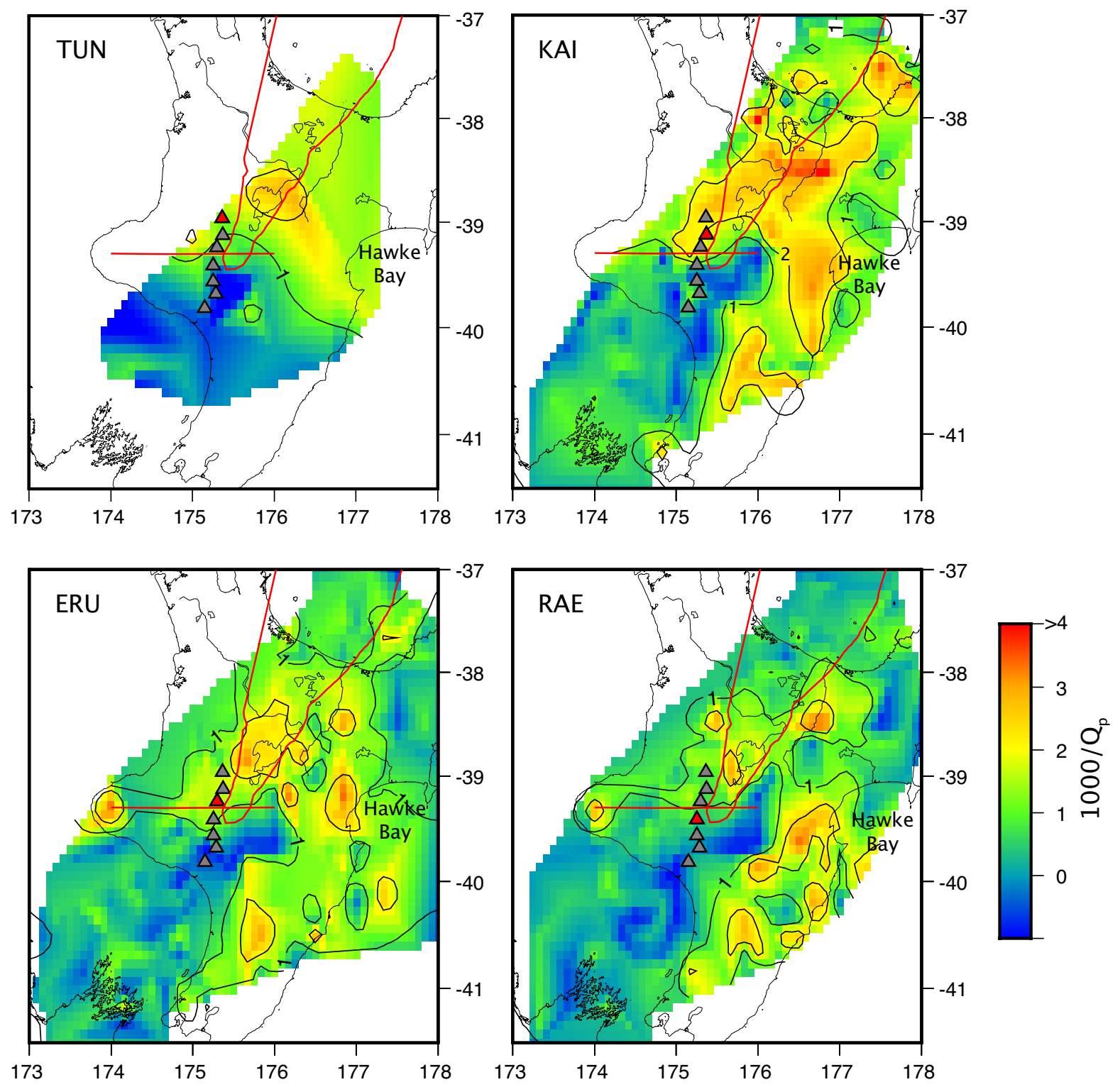

Figure 3.15: Northern TRAP stations $Q_{P}^{\prime}$ results. TRAP stations are shown as triangles the red triangle indicates the station used in each plot. Weighted mean values of $1000 / Q_{P}^{\prime}$ are contoured. Two contour lines are shown; the first is for $1000 / Q_{p}^{\prime}=1$ and the second is for $1000 / Q_{p}^{\prime}=2$. The red line running $\mathrm{E}-\mathrm{W}$ indicates the location of the change in crustal thickness seen in the receiver functions. Also shown in red is the boundary of the CVR. 

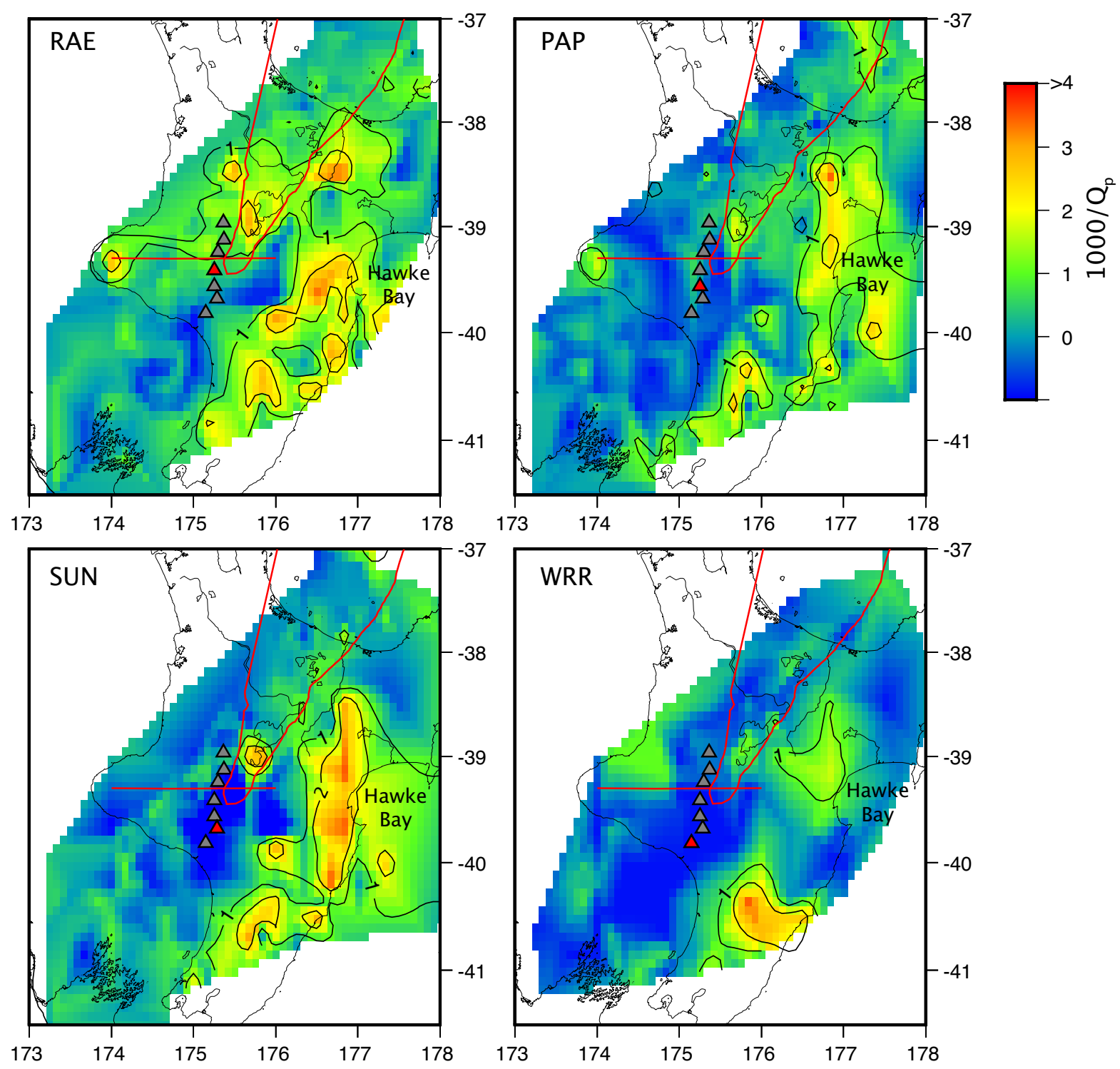

Figure 3.16: Southern TRAP stations $Q_{P}^{\prime}$ results. TRAP stations are shown as triangles the red triangle indicates the station used in each plot. Weighted mean values of $1000 / Q_{P}^{\prime}$ are contoured. Two contour lines are shown; the first is for $1000 / Q_{p}^{\prime}=1$ and the second is for $1000 / Q_{p}^{\prime}=2$. The red line indicates the location of the change in crustal thickness seen in the receiver functions. Also shown in red is the boundary of the CVR. 


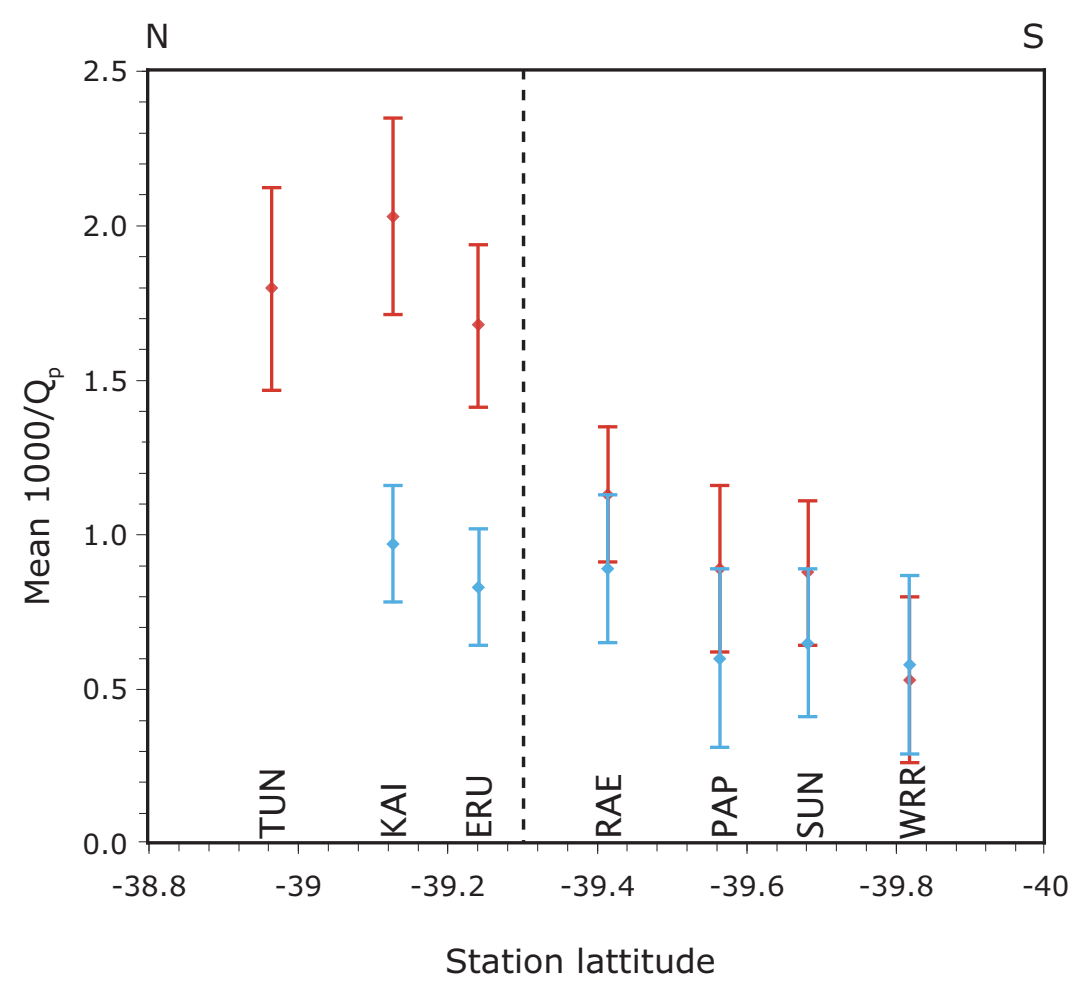

Figure 3.17: Mean attenuation for TRAP stations. Red diamonds show the normalised mean $1000 / Q_{p}^{\prime}$ values for events north of the Taranaki-Ruapehu line observed at each station. The blue diamonds show the normalised mean $1000 / Q_{p}^{\prime}$ values for events from the low attenuation region in the southwest of the North Island. Error bars are for the 95\% confidence level. The dashed line indicates the approximate latitude of the change in crustal thickness seen in the receiver functions.

shortened operational time of this station meant it had only 7 events from the south west in common with station RAE. The second station group includes the four stations south of the change in crustal thickness (RAE, PAP, SUN and WRR). Stations RAE, PAP, and SUN recorded 47 common events from the southwest region. Events recorded at station WRR returned many negative $Q_{p}^{\prime}$ values and consequently this station only had 18 in common with the other stations in the group, which were suitable for this analysis. The mean $1000 / Q_{p}^{\prime}$ for station RAE was compared for all station sets and mean values were multiplied by a normalisation factor that made the values of mean $1000 / Q_{p}^{\prime}$ constant for station RAE for both station groups. For the southern group of station the mean values of $1000 / Q_{p}^{\prime}$ for station RAE was 0.9 and 0.67 for the northern set of stations. For southwestern events the southern station group has been used as a reference since raypaths for event-station pairs in this group are entirely within the region of low attenuation. The mean values for the northern stations have therefore been multiplied a normalisation factor of 1.34 . For the mean $1000 / Q_{p}^{\prime}$ values and normalisation factors see appendix C C.1.

The second set of events used were from the region north of the change in crustal thickness. The same station groups were used. Attenuation results for station TUN 
included 14 events in common with the other northern stations and this station has been included in the analysis of this set of events. Stations KAI, ERU and RAE had 135 events in common, while stations the southern station group had 56 events in common. The mean $1000 / Q_{p}^{\prime}$ value for station RAE for the southern station group was 1.20 and for the northern station group it was 1.13. For this set of events the northern station mean $1000 / Q_{p}^{\prime}$ value for station $\mathrm{RAE}$ is taken as the reference value and the mean values for the southern stations are multiplied by 0.94 .

The resulting mean values of $1000 / Q_{p}^{\prime}$ for each station give a representative view of how attenuation varies along the line of the seismic stations. For stations KAI-WRR the mean values of $1000 / Q_{p}^{\prime}$ for events from the south west are between 0.5 and 1 (figure 3.17). For these events the northern stations exhibit slightly higher values but are within error of the southern stations. There is a larger contrast in station means for events originating from the north. For these events the mean $1000 / Q_{p}^{\prime}$ for stations north of the TRL range from 1.68-2.03. This corresponds to $Q_{p}^{\prime}$ values of 500-600, giving a similar result to that observed for the western North Island in the CNIPSE data. For stations south of the TRL $1000 / Q_{p}^{\prime}$ value drops quickly to values similar to those seen for events from the southwest. The sudden drop in attenuation may be in part due to slab ray paths, which will not sample the mantle below the northwestern North Island. The slab beneath these stations is shallower (50-60 km [Ansell and Bannister 1996]) than for stations in the north and slab paths for events deeper than this are likely to be longer. It can therefore be assumed that the mantle sampled by these station-event pairs is south of the TRL and hence the mean values of the northern events at these stations give similar results as southern events.

\subsection{Interpretation}

North Island New Zealand can be split up into four distinct attenuation regions; a region of extremely high attenuation below the $\operatorname{CVR}\left(1000 / Q_{p}^{\prime}=5.0 \pm 0.5\right)$, a region of intermediate attenuation below the northwestern North Island $\left(1000 / Q_{p}^{\prime}=1.9 \pm 0.5\right)$, a region of low attenuation below the southwestern North Island $\left(1000 / Q_{p}^{\prime} \leqslant 1\right)$ and a region with patches of high attenuation along the east coast $\left(1000 / Q_{p}^{\prime} \geqslant 2\right)$.

In order to compare the values of $Q_{p}^{\prime}$ obtained for each region to other studies and global norms, the effect of assuming the quality factor is independent of frequency must first be examined. Generally frequency dependence cannot be determined from field data. This is certainly the case for this data set. Figure 3.18 shows the data from figure 3.12 fitted for $\alpha=0.27$ and $\alpha=0.5$. As discussed previously, $\alpha=0.27$ is appropriate for melt free mantle materials and $\alpha=0.5$ is a maximum value expected for $2 \%$ melt over the frequency range of this study [Faul and Jackson 2005, Jackson et al. 2004]. Data can be fit equally as well using this range of $\alpha$, but $Q$ values must 
be reduced by a factor of 2.3 and 5.2 for $\alpha$ vales of 0.27 and 0.5 respectively. Assuming $Q$ is independent of frequency causes $Q$ values to be overestimated for $\alpha<1$.
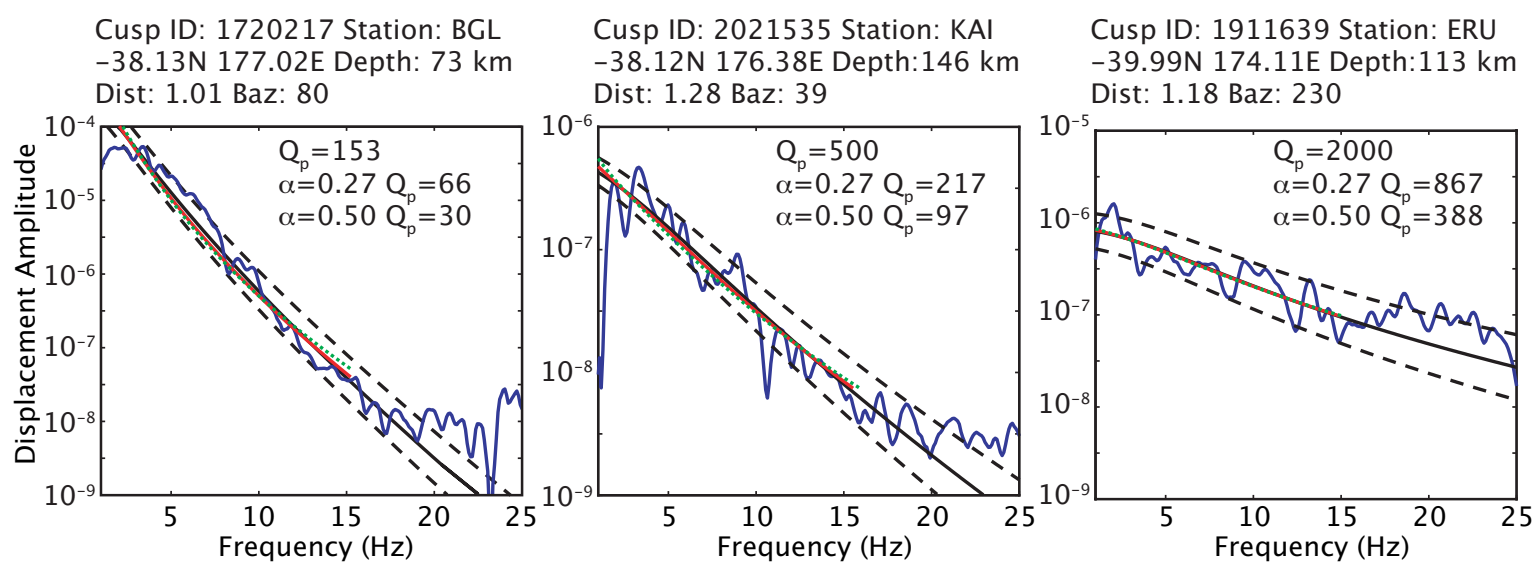

Figure 3.18: Fit to data using different frequency dependence values. Spectral data are shown as solid blue lines. For each spectra the solid black line shows the data fit for the best fit $Q_{p}$ value assuming no frequency dependence and the dashed black lines indicate upper and lower limits of the $95 \%$ confidence interval. The red line and the green line show the data fit between 1 and $15 \mathrm{~Hz}$ for frequency dependent $Q_{p}$ with $\alpha=0.27$ and $\alpha=0.5$ respectively. $Q_{p}$ values at a reference frequency of $1 \mathrm{~Hz}$ are also shown for the frequency independent case and the two frequency dependent cases.

At a reference frequency of $1 \mathrm{~Hz}$ typical $Q_{p}$ values for normal lithospheric mantle are $\sim 1300$ (see typical $Q$ values in section 3.2). $Q_{p}^{\prime}$ values for the CVR, northwestern North Island and the eastern North Island are at least a factor of 2 smaller than this even if frequency dependence is not taken into account.

Earthquakes from the eastern North Island are from depths of less than $75 \mathrm{~km}$ and sample the fore-arc region (figure 3.5). Patches of high attenuation have previously been observed along the southeastern margin of New Zealand in regions where the interseismic coupling between the overriding Australian plate and the subducting Pacific plate are weak [Eberhart-Phillips et al. 2005, Satake and Hashida 1989]. These regions have been associated with over-pressured subducted sediments. The high attenuation patch close to Hawke Bay correlates with a region where there is low plate coupling and the patch at $40.5 \mathrm{~S}$ correlates with the southern extent of low plate coupling coefficients [Wallace et al. 2004].

For the CVR the constant low $Q_{p}^{\prime}$ over the depth range 0-220 km (figure 3.14) suggests high attenuation in this region persists for the full depth of the mantle wedge. For the northwestern North Island the increasing $Q_{p}^{\prime}$ with depth observed in the CNIPSE data indicate that the source of the low $Q_{p}^{\prime}$ values lies within the mantle lithosphere. For these two regions there are two possible explanations for the low $Q_{p}$ values observed. The first is the presence of water [Karato 2003] and the second is elevated temperatures [Sato et al. 1989]. The back-arc setting and surface volcanism mean it is likely that 
both factors contribute to the observed high attenuation.

\subsubsection{Temperature, melt, water and $\mathrm{Q}$}

Various laboratory experiments have been conducted to determine the empirical relationship between attenuation and temperature in mantle materials. The recent experiments of Faul et al. [2004], Jackson et al. [2004] provide empirical relationships between $Q_{s}$ and temperature using an Andrade-pseudoperid model (equations C.0.1a-C.0.1h), which take into account the effects of pressure, grain size, frequency and the presence of melt. An increase in attenuation is observed at temperatures approaching melting temperatures $(\mathrm{Tm})$.

Little laboratory work has been done to determine empirical relationships between attenuation and water content. However regions in the earth where high water contents in the mantle are expected (e.g. in the mantle wedge above subduction zones) are often associate with high attenuation [e.g. Roth and Wiens 1999, Shito et al. 2006]. Karato [2003; 2006] describes theoretical relationships based on the experimentally observed enhancement of diffusion creep and grain boundary processes caused by water. Faul et al. [2004] conclude that grain boundary sliding accommodated by diffusion creep is the major mechanism contributing to attenuation at high temperatures, providing additional support to the Karato's assumptions. Sato et al. [1989] relate $Q_{p}$ to homologous temperature $(T / T m)$ and suggest that both melting and attenuation properties have a common origin in peridotite.

Figure 3.19 shows the empirical relationship between $Q_{s}$ and homologous temperature at a frequency of $1 \mathrm{~Hz}$ adapted from Jackson et al. [2004] (for equations see appendix C). Curves for both melt free and melt bearing olivine are shown at a pressure of 1 GPa. This pressure is representative of conditions just below the crust/mantle boundary for the CVR and the northwestern North Island. Homologous temperatures have been calculated assuming a dry melting temperature of $1220^{\circ} \mathrm{C}$ [e.g. Hirth and Kohlstedt 1996, Takahashi and Kushiro 1983]. The melt bearing and melt free relationships converge at $\sim T / T m=0.85$, therefore the melt free relationship is appropriate for $T / T m \leqslant 0.85$ and the melt bearing relationship should be used for $T / T m>0.85$.

$Q_{p}^{\prime}$ results are converted to equivalent $Q_{s}$ values at a reference frequency of $1 \mathrm{~Hz}$ using equation 3.2.7. Initially the melt free frequency dependence $(\alpha=0.27)$ is assumed. For the CVR the estimated $Q_{s}(\alpha=0.27)$ value is 40 . From figure 3.19 this low $Q_{s}$ value is indicative of temperatures well in excess of the solidus $(T / T m>1)$ and therefore the $Q_{s}$ value is recalculated using the melt bearing frequency dependence $(\alpha=0.5)$. For melt bearing olivine, $Q_{s}(\alpha=0.5)$ at the reference frequency of 1 $\mathrm{Hz}$ is 18 , which is indicative of temperatures just above melting $(T / T m=1.02)$ and the presence of 1-2\% melt volume [Sato et al. 1989]. Back-arc settings such as the 


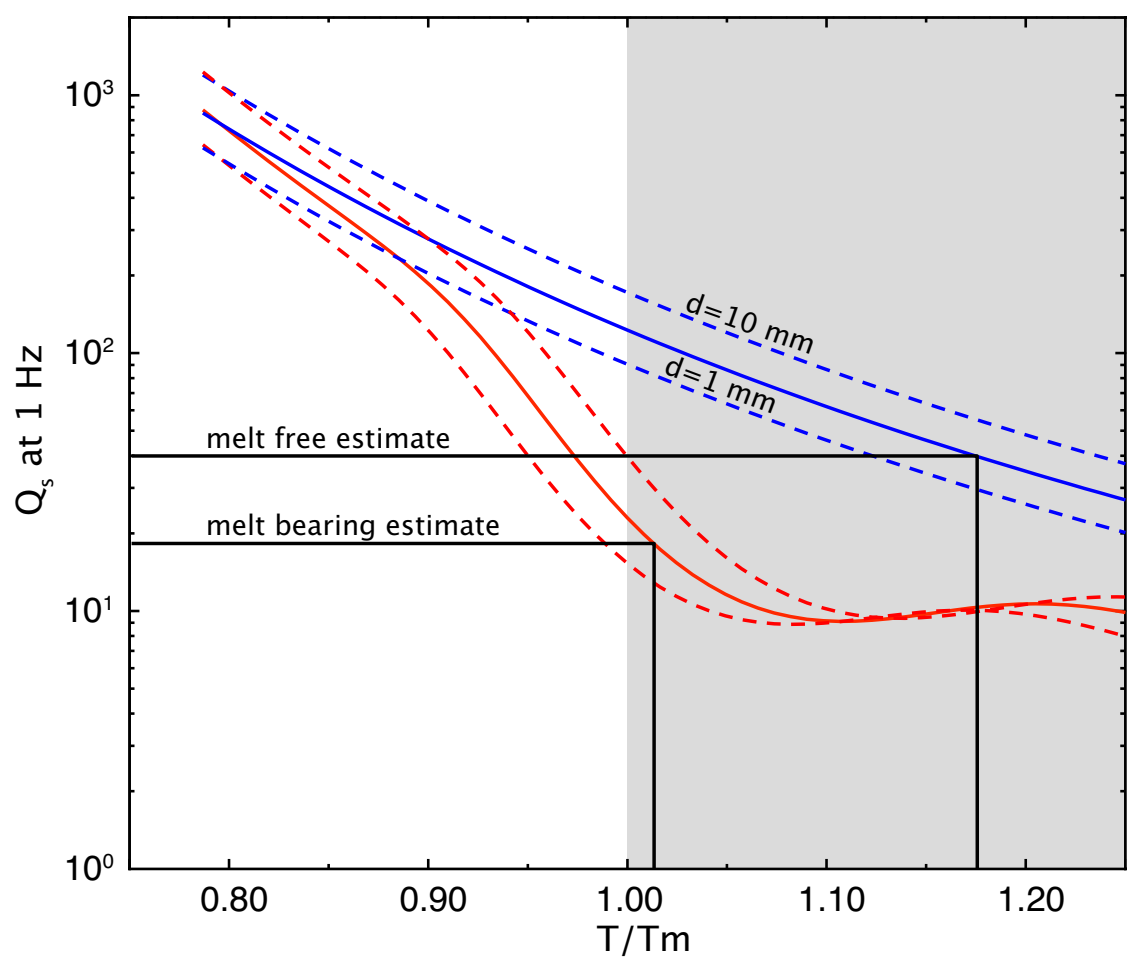

Figure 3.19: Homologous temperature vs $Q_{s}$ adapted from equations of Jackson et al. [2004]. The solid blue line show the relationship between temperature and $Q_{s}$ for melt free olivine with a $3 \mathrm{~mm}$ grain size. The solid red line shows the relationship between attenuation and $Q_{s}$ for a $1 \%$ partial melt at $1 \mathrm{GPa}$. The dashed lines show limits of estimated mantle grain sizes $1-10 \mathrm{~mm}$. Solid black lines show the T/Tm estimates for the CVR for both the melt free and melt bearing cases.

CVR are usually associated with hydrated mantle wedges. The presence of water has the effect of reducing the melting temperatures. For a water saturated mantle the melting temperature is reduced to $970^{\circ} \mathrm{C}$ [Green 1973 , Iwamori 1998] and $\mathrm{T} / \mathrm{Tm}=1.02$ therefore equivalent to an absolute temperature of $990^{\circ} \mathrm{C}$.

For the northwestern North Island, melt free $Q_{s}(\alpha=0.27)$ at the reference frequency is estimated to be 100. This corresponds to a temperature just above melting if the melt free relationship is used, suggesting that the melt bearing relationship should also be used for this region. $Q_{s}$ recalculated using the melt bearing $\alpha$ is 46 , which corresponds to a temperature just below melting $(T / T m=0.97)$. Although this indicates the absence of melt the melt bearing relationship still holds for $T / T m>0.85$. Assuming water saturated conditions $T / T m=0.97$ is equivalent to an absolute temperature of $940^{\circ} \mathrm{C}$. This temperature is consistent with estimates of $800-950^{\circ} \mathrm{C}$ at based on heatflow data for the region [Pandey 1981a]. 


\section{Chapter 4}

\section{MAGNETOTELLURICS}

\subsection{Magnetotellurics abstract}

Magnetotellurics (MT) is a passive source technique that uses natural fluctuations in the Earth's magnetic field to determine the resistivity structure of the Earth. Resistivity decreases with temperature and water content. This makes magnetotellurics an ideal tool for investigating the Taranaki-Ruapehu Line (TRL) since this boundary is associated with elevated heat flow and a change in crustal thickness.

MT data were collected along a N-S line, paralleling the seismic stations used for the receiver function and attenuation chapters. MT data were inverted using a 2D smooth model code to provide a $2 \mathrm{D}$ resistivity profile. The resistivity profile reveals a low resistivity surface sediment layer $(<100 \Omega \mathrm{m})$, which deepens southward. Below this is a high resistivity crustal layer $(>1000 \Omega \mathrm{m})$. North of the TRL $\left(-39.3^{\circ}\right)$ there is a low resistivity anomaly $(100 \Omega \mathrm{m})$ within the mantle, which is coincident with a region of high attenuation (chapter 3). This resistivity anomaly indicates that the mantle north of the TRL must be hydrated and at elevated temperatures. Assuming water saturated peridotite, the mantle resistivity anomaly equates to a minimum temperature of $850^{\circ} \mathrm{C}$ at $30 \mathrm{~km}$ depth.

\subsection{MT Theory}

\subsubsection{Source Signal and Basic Principles}

MT uses external time variations in the geomagnetic field as a source signal. A changing magnetic field induces an orthogonal electric field that will produce a current in a conductor, in this case the earth. By measuring time variations in electrical potential at the surface of the Earth and time variations in the magnetic field, the resistivity of the earth can be determined.

Most of the Earth's magnetic field is generated internally, as are very long wavelength variations (eg. secular variation). Shorter wavelength fluctuations in the geomagnetic field are of external origin and are caused by the interaction of the solar wind with 


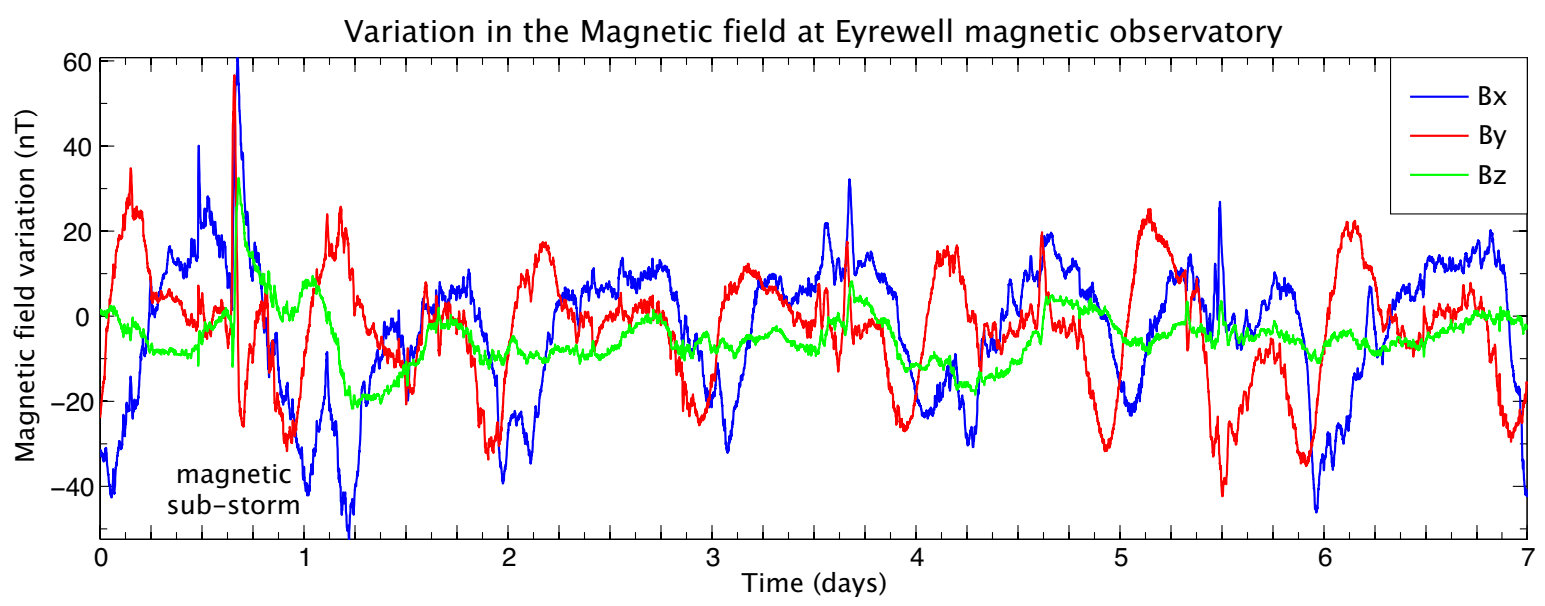

Figure 4.1: 3-component magnetic field variations at the Eyrewell magnetic observatory New Zealand [GNS Science 2002] over the time of our survey (26/08/2002 to 01/09/2002). Measurements are recorded every minute.

the Earth's magnetic field and by distant thunderstorm activity. Figure 4.1 shows the 3-component variation in the magnetic field at New Zealand's magnetic observatory over the time period of this study [GNS Science 2002]. Externally derived geomagnetic fluctuations have a broad frequency range (figure 4.2). MT utilises frequencies from 0.0001 to about $10000 \mathrm{~Hz}$. Generally fluctuations $\geq 1 \mathrm{~Hz}$ are caused by lightning discharges, the energy from which propagates around the Earth in the ionospheric cavity. Fluctuations $<1 \mathrm{~Hz}$ are caused by the solar wind, which can deflect and compress the geomagnetic field, inject plasma into the ionosphere and induce currents in the ionosphere. These sources constantly provide a signal for MT surveys.

MT uses the assumptions that the source field is a plane wave normally incident to the $z$ direction. This means that the source signal has only components in the $x$ and $y$ directions. This assumption is valid for frequencies $<1 \mathrm{~Hz}$ but is only valid for frequencies $\geq 1 \mathrm{~Hz}$ if the source is distant. For this reason it is not ideal for lightning sources to be close to the MT site, in fact most of the lightning energy comes from equatorial lightning belts.

The basic theory for MT is that of electromagnetic induction and is based on Maxwell's equations, which describe the behaviour of electromagnetic waves [Maxwell 1865].

$$
\begin{aligned}
\nabla \times \underline{B} & =\mu_{0} \underline{J}+\mu_{0} \varepsilon_{0} \frac{\partial \underline{E}}{\partial t} \\
\nabla \times \underline{E} & =-\frac{\partial \underline{B}}{\partial t} \\
\nabla \cdot \underline{E} & =\frac{q}{\varepsilon_{0}} \\
\nabla \cdot \underline{B} & =0
\end{aligned}
$$




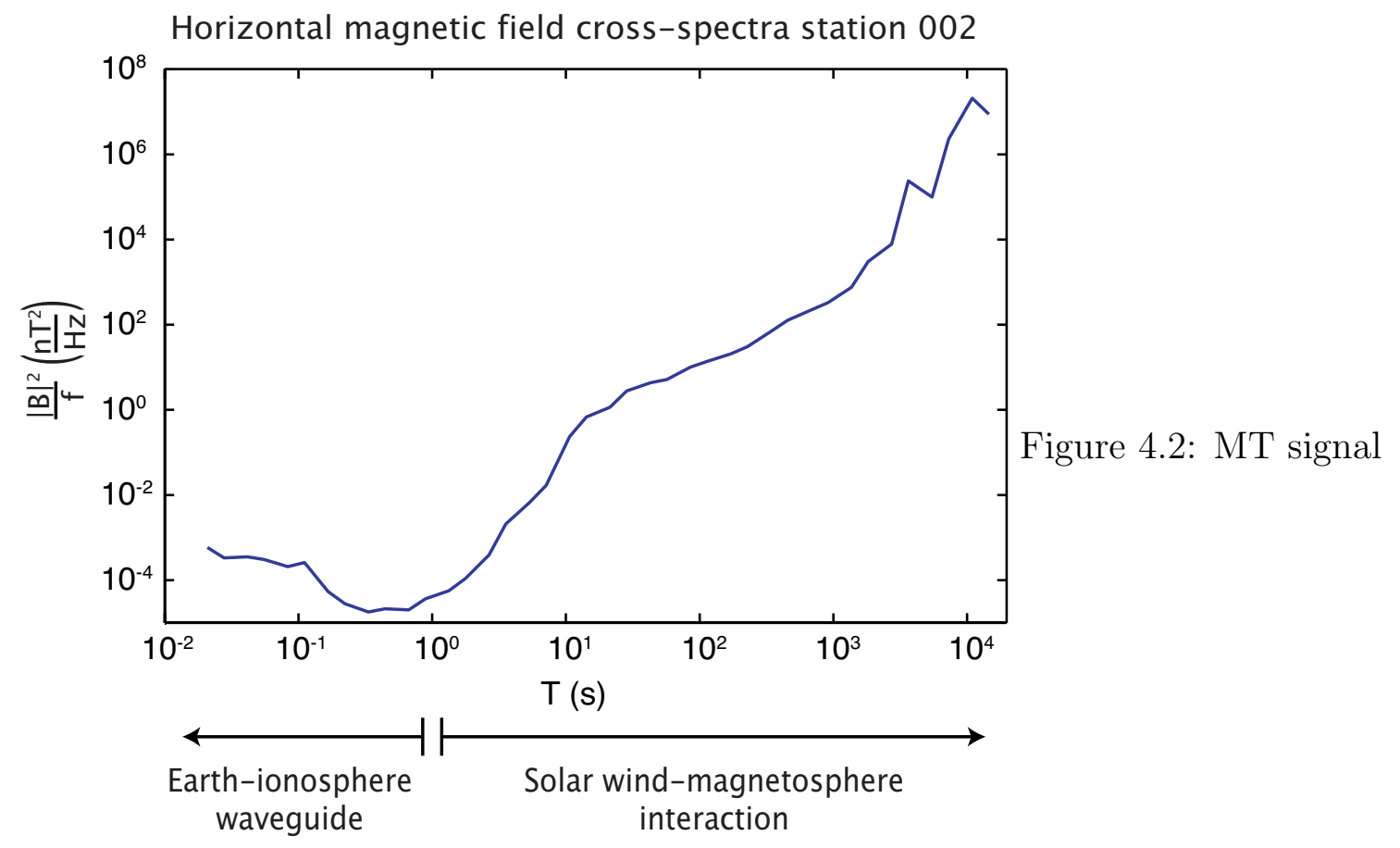

Where $\underline{B}$ is the magnetic field, $\underline{E}$ electric field, $\underline{J}$ is the electric current density, $q$ is a volume density of charge, $\mu_{0}$ is the permeability of free space $\left(4 \pi \times 10^{-7} \mathrm{~Wb} / \mathrm{Am}\right)$ and $\epsilon_{0}$ is the permittivity of free space $\left(8.8542 \times 10^{-12} \mathrm{C}^{2} / \mathrm{Nm}^{2}\right)$. A harmonic time dependence is assumed for $\underline{B}$ and $\underline{E}$ in the form $e^{i \omega t}$, where $\omega$ is the angular frequency. Equation 4.2 .1 can be simplified using Ohm's law to relate the $\underline{J}$ to $\underline{E}$.

$$
\underline{J}=\sigma \underline{E}
$$

Where $\sigma$ is the electrical conductivity ( $\sigma=1 / \rho$, where $\rho$ is the resistivity). The second term on the right of 4.2.1 represents the displacement current. This term is small for the frequency and resistivity range considered for MT and can therefore be neglected.

By substituting 4.2.5 into 4.2.1 and taking the curl, it can be demonstrated that the time varying magnetic induction, $\underline{B}$, obeys the diffusion equation.

$$
\begin{aligned}
\nabla^{2} \underline{B} & =\mu_{0} \sigma \frac{\partial \underline{B}}{\partial t} \\
& =\mu_{0} \sigma i \omega \underline{B}
\end{aligned}
$$

Taking the curl of 4.2.2 gives:

$$
\begin{aligned}
\nabla^{2} \underline{E} & =\mu_{0} \sigma \frac{\partial \underline{E}}{\partial t}+\nabla\left(\frac{q}{\epsilon_{0}}\right) \\
& =\mu_{0} \sigma i \omega \underline{E}+\nabla\left(\frac{q}{\epsilon_{0}}\right)
\end{aligned}
$$


Where the second term on the right arises from accumulation of charge along boundaries. For a 1D earth this term will be zero.

\subsubsection{Skin depth}

Electromagnetic waves decay exponentially as they diffuse into a medium [Cagniard 1953]. The depth to which the time varying electromagnetic field penetrates the Earth can be estimated by considering a horizontally oscillating $\underline{B}$ field interacting with a conducting half space with the general form:

$$
\underline{B}=B_{0} e^{-\alpha z} e^{i \omega t}
$$

Substituting 4.2 .8 into 4.2 .6 and constraining $\alpha$ to be positive, since $\underline{B}$ must decay with depth, gives:

$$
\alpha=\sqrt{\frac{\omega \mu_{0} \sigma}{2}}+i \sqrt{\frac{\omega \mu_{0} \sigma}{2}}
$$

and

$$
\underline{B}=\underline{B}_{0} e^{-z / \delta} e^{i(\omega t-z / \delta)}
$$

where

$$
\delta=\sqrt{\frac{2}{\omega \mu_{0} \sigma}}=\sqrt{\frac{2 \rho}{\omega \mu_{0}}}
$$

Within the conductor, $\underline{B}$ is thus a travelling wave with exponentially decaying amplitude. $\delta$ is the distance into the conductor over which the initial amplitude of $\underline{B}$ decays by a factor of $1 / e$ and is known as the skin depth. The skin depth formula gives us the important result, fundamental to MT, that depth of penetration is a function of both frequency and conductivity. Penetration depth increases with decreasing frequency and decreasing conductivity. The exponential decay of electromagnetic fields makes them insensitive to conductivity structure deeper than the skin depth, thus high frequency MT signals will sample only the shallow structure, while low frequency MT signals will sample deeper into the earth. As seen previously, the MT source signal contains a broad range of frequencies giving us a broad range of penetration depths.

\subsubsection{The impedance tensor}

In practice, a magnetotelluric sounding measures the variations in the magnetic and electric fields at the surface of the earth at many frequencies. The interaction of these two fields is a function of the resistivity structure as can be seen by combining equations 4.2.1 and 4.2.5. The relationship between $\underline{E}$ and $\underline{B}$ can be expressed in terms of an 
impedance tensor, $\underline{Z}$, where:

$$
\left[\begin{array}{c}
E_{x} \\
E_{y}
\end{array}\right]=\frac{1}{\mu_{0}}\left[\begin{array}{ll}
Z_{x x} & Z_{x y} \\
Z_{y x} & Z_{y y}
\end{array}\right]\left[\begin{array}{c}
B_{x} \\
B_{y}
\end{array}\right]
$$

By determining the components of the impedance tensor at a single frequency we can extract a volumetrically averaged resistivity (apparent resistivity, $\rho_{a}$ ) of the earth to the depth sampled by that frequency. The easiest way to illustrate this process is to look at the simplest case, that of a homogeneous half space.

\section{Homogeneous half-space}

For this case there are no vertical boundaries where charge may accumulate. The right hand side of equation 4.2.3 can be set to zero, as can the second term of 4.2.7. The electric field and both the primary and induced magnetic field will therefore only have components in the $x$ and $y$ directions. Along with the plane wave assumption this allows $\frac{\partial B_{z}}{\partial y}, \frac{\partial B_{z}}{\partial x}, \frac{\partial B_{y}}{\partial x}$ and $\frac{\partial B_{x}}{\partial y}$ to be set to zero and equations 4.2 .1 and 4.2 .5 give:

$$
\begin{aligned}
& -\frac{\partial B_{y}}{\partial z}=\mu_{0} \sigma E_{x} \quad \Rightarrow \quad \alpha B_{y} e^{-\alpha z} e^{i \omega t}=\mu_{0} \sigma E_{x} e^{-\alpha z} e^{i \omega t} \\
& \frac{\partial B_{x}}{\partial z}=\mu_{0} \sigma E_{y} \quad \Rightarrow \quad-\alpha B_{x} e^{-\alpha z} e^{i \omega t}=\mu_{0} \sigma E_{y} e^{-\alpha z} e^{i \omega t}
\end{aligned}
$$

Therefore, for the homogeneous half-space, $E_{x}$ is independent of $B_{x}, E_{y}$ is independent of $B_{x}$ and the $Z_{x x}$ and $Z_{y y}$ components of $\underline{Z}$ are zero. The two non-zero components of the impedance tensor can be defined as:

$$
\begin{aligned}
& Z_{x y}=\mu_{0} \frac{E_{x}}{B_{y}}=\sqrt{\frac{\mu_{0} \omega}{2 \sigma}}+i \sqrt{\frac{\mu_{0} \omega}{2 \sigma}} \\
& Z_{y x}=\mu_{0} \frac{E_{y}}{B_{x}}=-\sqrt{\frac{\mu_{0} \omega}{2 \sigma}}-i \sqrt{\frac{\mu_{0} \omega}{2 \sigma}}
\end{aligned}
$$

For this case $Z_{x y}=-Z_{y x}$. The same result can be obtained starting from equation 4.2.2. $\underline{Z}$ is complex and can therefore be expressed as a magnitude and phase angle $(\phi)$. The magnitude of the diagonal components of $\underline{Z}$ can be used to calculate the apparent resistivity (4.2.15) and in this case will be the true resistivity of the half-space. The real and imaginary parts of $\underline{Z}$ are equal in magnitude for the homogeneous half-space, therefore the phase angle is $45^{\circ}(4.2 .16)$.

$$
\begin{aligned}
\rho_{a, i j} & =\frac{1}{\sigma}=\left|Z_{i j}(\omega)\right|^{2} \frac{1}{\mu_{0} \omega} \\
\phi_{a} & =\arctan \left(\frac{\operatorname{Im} Z_{i j}}{\operatorname{Re} Z_{i j}}\right)
\end{aligned}
$$




\section{D layered earth}

For a half-space with $N$ layers (figure 4.3) the diffusion equation holds within each individual layer. An additional term must be added to the general solutions of equations 4.2.6 and 4.2.7 to allow for reflections from the lower boundary of the layer. For the electric field the general solution within the $n$th layer is:

$$
\begin{aligned}
E_{n} & =E_{1 n} e^{i \omega t-\alpha_{n} z}+E_{2 n} e^{i \omega t+\alpha_{n} z} \\
& =a_{n} e^{-\alpha_{n} z}+b_{n} e^{\alpha_{n} z}
\end{aligned}
$$

The second term on the right hand side represents the upward travelling reflected

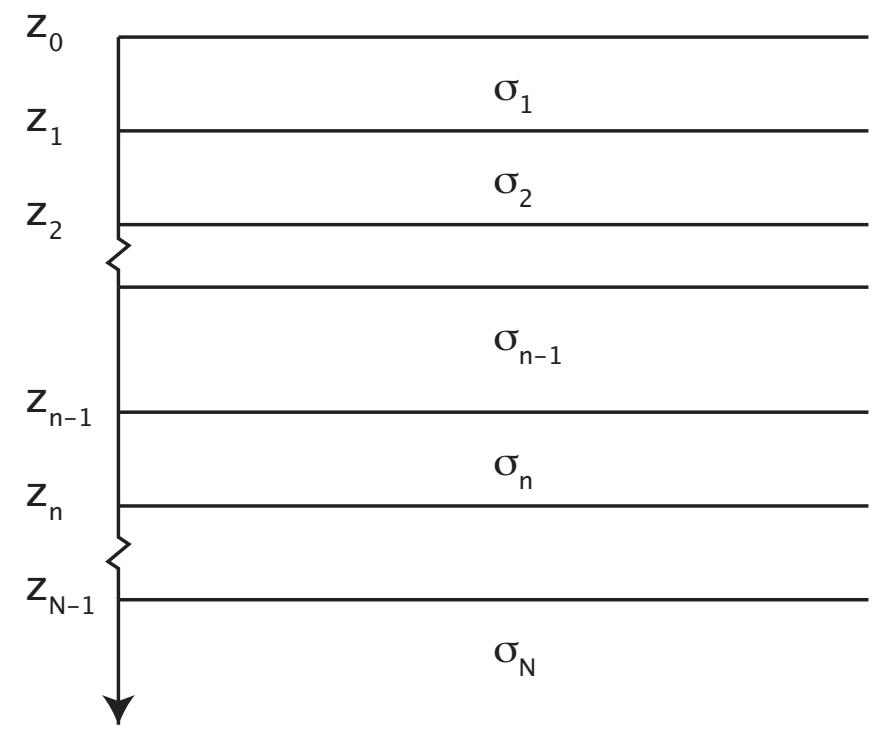

Figure 4.3: 1D layered earth

component and time dependent components are incorporated into the coefficients $a_{n}$ and $b_{n}$. Substituting 4.2.17 and 4.2.5 into equation 4.2.1 gives $B_{y n}$ and $B_{x n}$ in terms of $a_{n}$ and $b_{n}$ :

$$
\begin{aligned}
& B_{y n}=\frac{\alpha_{n}}{i \omega}\left[a_{n} e^{-\alpha_{n} z}-b_{n} e^{\alpha_{n} z}\right] \\
& B_{x n}=\frac{\alpha_{n}}{i \omega}\left[-a_{n} e^{-\alpha_{n} z}+b_{n} e^{\alpha_{n} z}\right]
\end{aligned}
$$

From these equations expressions for $\underline{Z}$ at the top and bottom of the $n$th layer can be derived. As for the homogeneous model $Z_{x x}$ and $Z_{y y}$ are zero, since there are no vertical boundaries where charge may accumulate, and $Z_{x y}=-Z_{y x}$.

At the boundary between the $n$th and the $(n+1)$ th layer the components of the magnetic and electric fields must be continuous. By using these continuity conditions an expression for $Z_{n}$ at $z_{n-1}$ can be derived in terms of $Z_{n+1}$ at $z_{n}, \alpha_{n}$ and the thickness 
of layer $n$ :

$$
Z_{n}\left(z_{n-1}\right)=\frac{1}{\alpha_{n}} \frac{\frac{\alpha_{n}}{i \omega} Z_{n+1}\left(z_{n}\right)+\tanh \left[\alpha_{n} d_{n}\right]}{1+\frac{\alpha_{n}}{i \omega} Z_{n+1}\left(z_{n}\right) \tanh \left[\alpha_{n} d_{n}\right]}
$$

where $d_{n}$ is the thickness of layer $n$. This equation is known as Wait's recursion formula and can be used to calculate the surface impedance of any layered half-space. From the surface impedance the apparent resistivity is calculated as for the homogeneous half-space (equation 4.2.15). This value now represents the resistivity of an equivalent half-space and is a volumetrically averaged resistivity. The change in resistivity with depth is reflected in the change in apparent resistivity with change in frequency. This is a result of the penetration depth.

The phase of the impedance provides additional constraints on the resistivity; Kunetz [1976] showed that for a 1D layered earth the surface impedance is a minimum phase function. This result implies that the magnitude and phase of the impedance are not independent and can be related through the Hilbert transform. Weaver [1994] shows that, to a first order approximation, the phase is related to the apparent resistivity by:

$$
\phi(T) \approx \frac{\pi}{4}-\frac{\pi}{4} \frac{d \log \rho_{a}}{d \log T}
$$

or the phase will be $>45^{\circ}$ where $\rho$ is decreasing with depth and $<45^{\circ}$ where $\rho$ is increasing with depth.

\section{$2 \mathrm{D}$ structures}

For a $2 \mathrm{D}$ structure it is advantageous to rotate the co-ordinate system so that the $x$ and $y$ axis are respectively parallel and perpendicular to the strike of the structure. In this reference frame the first two of Maxwell's equations (4.2.1 and 4.2.2) can be separated into two independent systems. The first is for the electric field parallel to the strike of the structure:

$$
\begin{aligned}
\frac{\partial B_{z}}{\partial y}-\frac{\partial B_{y}}{\partial z} & =\mu_{0} \sigma E_{x} \\
\frac{\partial E_{x}}{\partial z} & =-i \omega B_{y} \\
\frac{\partial E_{x}}{\partial y} & =i \omega B_{z}
\end{aligned}
$$


This is called the E-polarisation or the Transverse Electric (TE) mode. The second system is for the magnetic field parallel to the strike of the structure:

$$
\begin{aligned}
\frac{\partial E_{z}}{\partial y}-\frac{\partial E_{y}}{d z} & =-i \omega B_{x} \\
\frac{\partial B_{x}}{\partial z} & =\mu_{0} \sigma E_{y} \\
\frac{\partial B_{x}}{\partial y} & =-\mu_{0} \sigma E_{z}
\end{aligned}
$$

This is called the B-polarisation or the Transverse Magnetic (TM) mode. In this reference frame the impedance tensor take the form:

$$
\left[\begin{array}{c}
E_{x} \\
E_{y}
\end{array}\right]=\frac{1}{\mu_{0}}\left[\begin{array}{cc}
0 & Z_{x y} \\
Z_{y x} & 0
\end{array}\right]\left[\begin{array}{c}
B_{x} \\
B_{y}
\end{array}\right]
$$

$Z_{x y}$ and $Z_{y x}$ are no longer equal in magnitude. Where the strike of the $2 \mathrm{D}$ structure is not known it can be found by rotating the impedance tensor to minimise $Z_{x x}$ and $Z_{y y}$ [e.g. Simpson and Bahr 2005, pg. 81]. A phase and apparent resistivity can be calculated for each of the non-zero terms of the impedance tensor.

Unlike the 1D case there is no analytical solution to Maxwell's equations for 2D structures and numerical solutions are sought using finite difference or finite element methods.

\section{Distortions}

Magnetotelluric data can be distorted by localised near surface heterogeneities and topography. Distortions caused by small-scale conductivity anomalies close to the surface are called galvanic distortions. The simplest of these distortions has the effect of shifting the entire apparent resistivity data up or down and are known as static shifts. Static shifts can be removed easily using some reference resistivity. Often DC resistivity or TEM data are used as a near surface (short period) reference. More complex galvanic distortions require more complex removal methods [Bibby et al. 2005, Groom and Bailey 1989, Lilley 1998].

\section{$3 \mathrm{D}$ structure}

For 3D structures, Maxwell's equations cannot be simplified and all components of the impedance tensor are non-zero. As for the 2D case there is no analytical solution. 


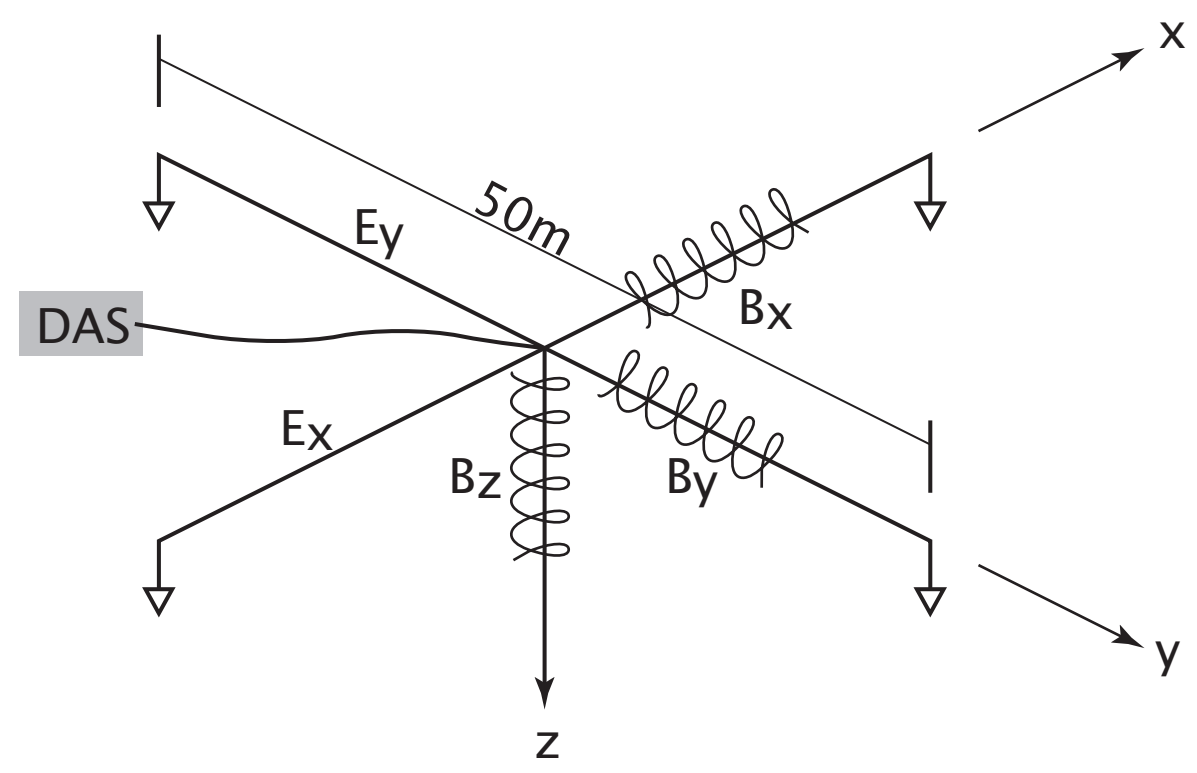

Figure 4.4: Typical MT station layout. Electrodes are shown as inverted triangles and induction coils are shown as coils. Electrodes and coils are connected to a data acquisition system (DAS) along with a GPS antenna for timing. For this study the $\mathrm{x}$-axis was oriented to magnetic north.

\subsection{MT Data}

\subsubsection{Data acquisition}

In practice both the source field (incident magnetic field) and the induced field (electric field and induced magnetic field) are measured at the surface of the earth. Figure 4.4 shows a typical layout for a MT station. The magnetic field is measured using induction coils and the electric field is determined by measuring the potential difference between two electrodes at the surface. For a $1 \mathrm{D}$ earth it is sufficient to measure the magnetic field variations in the horizontal plane $(x y)$ however $2 \mathrm{D}$ and $3 \mathrm{D}$ structures may induce a vertical component in the magnetic field and additional information can be gained from measuring this component. Only the horizontal component of the electric field is measured since the large conductivity difference between the air and the Earth means that the vertical electric field will be very small. Sites are preferably located away from cultural electrical noise such as electric fences, power lines and electrified rail lines.

Data for this study were collected in conjunction with GNS Science using three of their Phoenix Systems. This system consists of a MTU-5 data acquisition system (DAS), a GPS antenna, three MTC-50 magnetic sensor coils and PE-4 non-polarising electrodes. This set-up can be used to record data with a frequency range of 0.00002-400 Hz. Data were recorded at six sites located at approximately $20 \mathrm{~km}$ spacings in a N-S line, which parallels the line of seismic stations (figure 4.5 and appendix A table A.3).

The remote locations of sites in this survey ensured that sites could generally be located 


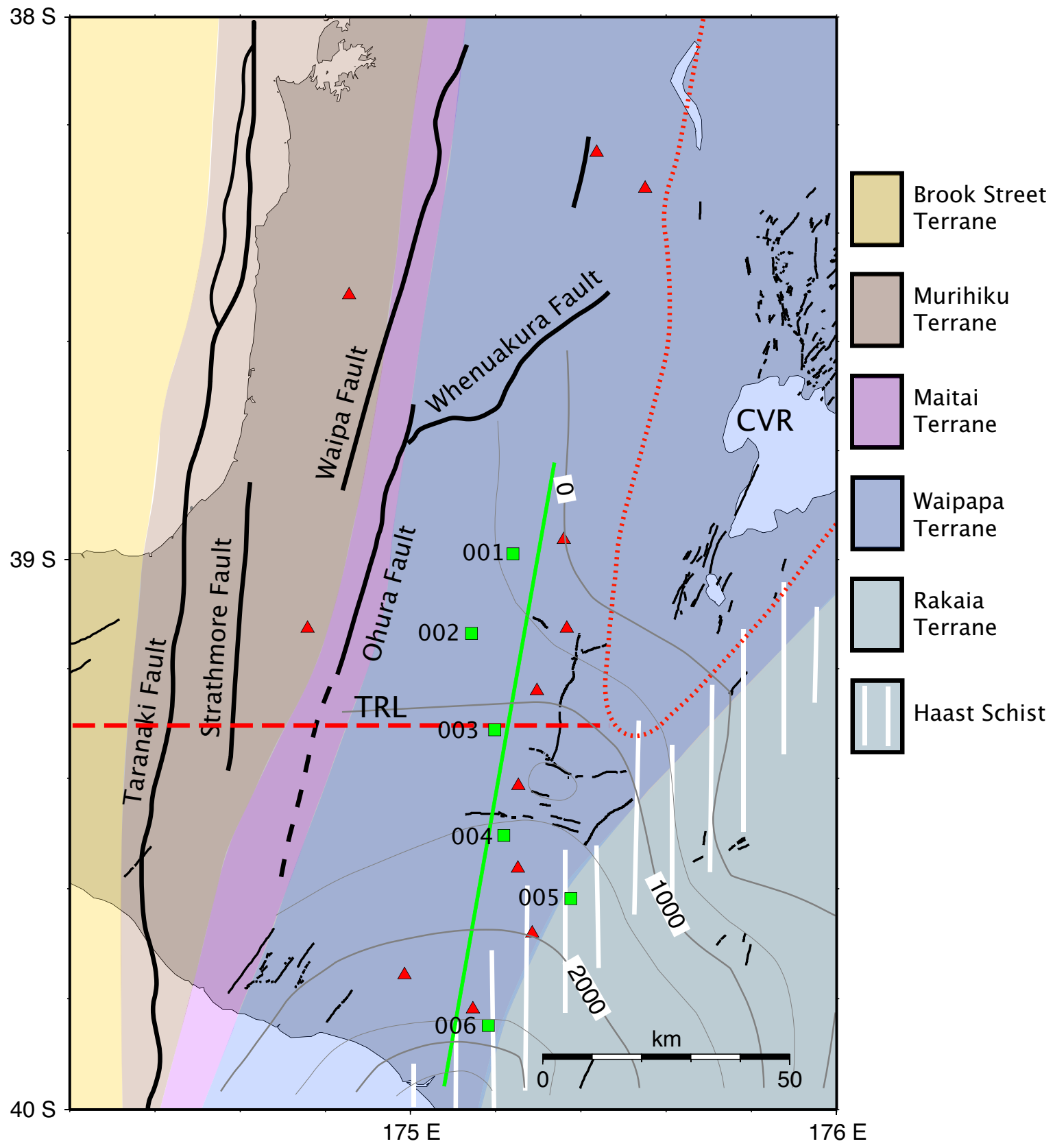

Figure 4.5: Stations location. MT stations used for this study are shown as green squares and seismic stations as red triangles. The green line through the stations is the profile used for 2D modelling. The boundary of the Central Volcanic Region (CVR) is shown as a red dotted line and the Taranaki-Ruapehu line is shown as a red dashed line. Thin black lines indicate active faulting and thick black lines indicate large-scale faults. Basement contours shown in grey are relative to mean sea level. Basement terranes and tectonic overprinting are indicated as per the key. 
away from most electrical noise. At some sites electric fences could not be avoided but arrangements were made for them to be turned off for the duration of the survey. A 50 $\mathrm{Hz}$ notch filter was used to minimise any noise from power mains. Sites were chosen where the equipment could be deployed on reasonably flat lying land with a good sky view for GPS timing and location.

Stations were left to record data for up to 64 hours, however GNS Science was in the initial stages of testing this equipment and two of the stations failed at some point. The northern most station recorded for less than 12 hours and the southern most station recorded for only 44 hours. The shorter recording times limit the useable frequency range of the data (see figure 4.14). The strength of the incident magnetic field signal varies with time depending mainly on magnetic storm activity. The Disturbance stormtime index (Dst) is a measure of global magnetic storm activity. A normal baseline value of the Dst index is approximately -20 $\mathrm{nT}$ and decreases with increasing magnetic storm activity [Gonzalez et al. 1994]. Dst data can be downloaded from the National Geophysical Data Center website [NOAA 2007]. Figure 4.6 shows the hourly Dst index for the time of the MT survey. This study was conducted over a 7-day period from $26 / 08 / 2002$ to $01 / 09 / 2002$. In the week prior to the field campaign there was intense magnetic storm activity. During the field campaign magnetic storm activity was relatively normal with three periods where sub-storm levels were reached. The sharp decrease in the Dst indices between hours 13 and 19 matches the sub-storm event identified in the Eyrewell observatory data (figure 4.1).

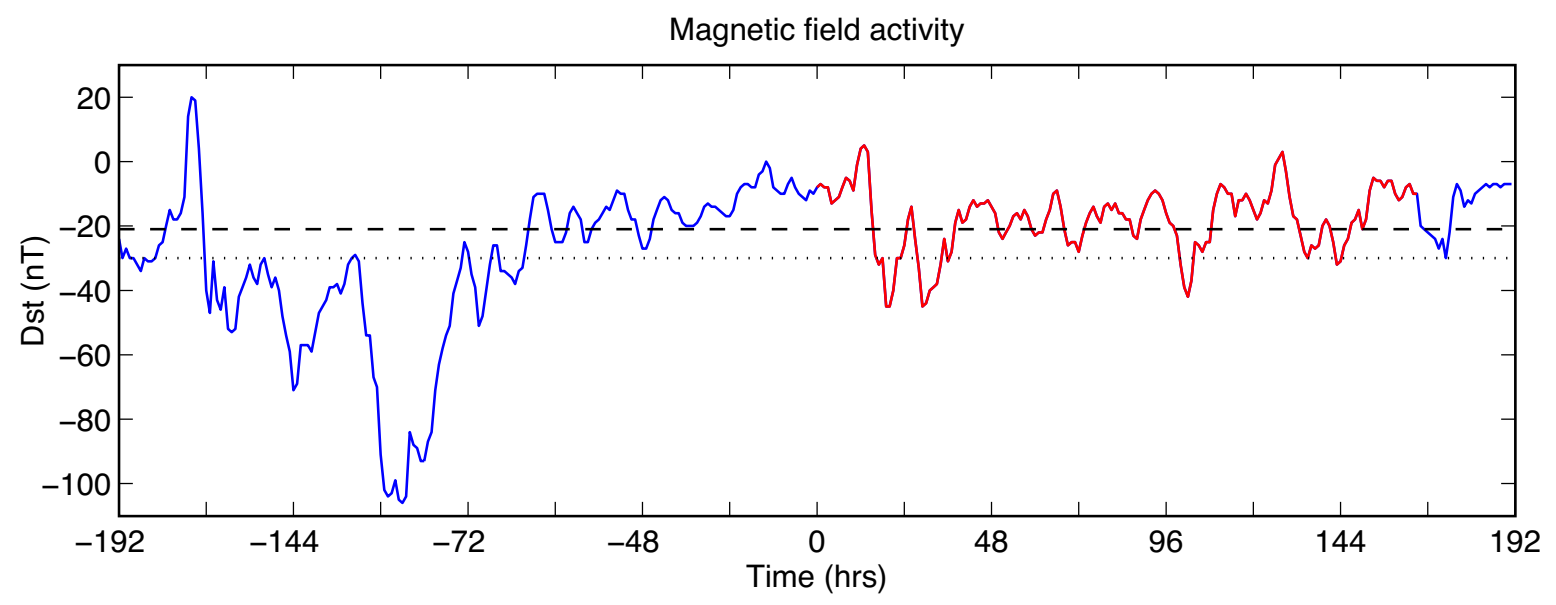

Figure 4.6: Magnetic storm activity. The solid blue line indicates the magnetic storm activity before and after the MT field campaign. The red line indicates the storm activity over the recording period. The dashed line indicates the mean Dst index for 2002 and the dotted line is at magnetic sub-storm level. 


\subsubsection{Processing}

Single site processing of the time domain data was conducted by GNS Science using Phoenix Geophysics proprietary software (SSMT). This processing includes the following steps:

1. Fourier transform. Spectra estimates are calculated using the cascade decimation technique [ Wight and Bostick 1980] over the frequency range 48-6.866 $\times 10^{-5}$ Hz. In order to avoid aliasing in the frequency domain a minimum of 6 evaluation frequencies are used for every decade of frequency. A constant percentage bandwidth was used to evaluate spectra at 40 frequencies evenly spaced along a log-frequency scale.

2. Calibration. Instrument responses were removed from the spectral data using calibration data.

3. Auto- and cross-power spectral estimates. Robust processing is used to determine auto- and cross -spectral estimates for the 5 channels. This process down-weights outliers in calculations of the mean estimates. At this stage minimum coherence values were set to $85 \%$. Twenty mean auto- and cross-power estimates are provided for each evaluation frequency for manual inspection. Obvious outliers are eliminated and remaining values are averaged.

4. Impedance tensor estimates. The final averaged auto and cross-power spectral estimates are used to calculate estimates of impedance. From equation 4.2.12:

$$
E_{x}=\frac{1}{\mu_{0}}\left[Z_{x x} B_{x}+Z_{x y} B_{y}+n\right]
$$

where $\mathrm{n}$ is due to random noise in the data. Taking cross-powers of this equation with $B_{x}$ and $B_{y}$ gives:

$$
\begin{aligned}
& <E_{x} B_{x}^{*}>=\frac{1}{\mu_{0}}\left[Z_{x x}<B_{x} B_{x}^{*}>+Z_{x y}<B_{y} B_{x}^{*}>+<n B_{x}^{*}>\right] \\
& <E_{x} B_{y}^{*}>=\frac{1}{\mu_{0}}\left[Z_{x x}<B_{x} B_{y}^{*}>+Z_{x y}<B_{y} B_{y}^{*}>+<n B_{y}^{*}>\right]
\end{aligned}
$$

where $B_{x}^{*}$ and $B_{y}^{*}$ are the complex conjugates of $B_{x}$ and $B_{y}$ respectively and $<>$ indicates the band averaged values calculated in step 3. Since $\mathrm{n}$ is random $<n B_{x}^{*}>$ and $<n B_{y}^{*}>\approx 0$. These two equations can be solved simultaneously 
to give estimates of $Z_{x x}$ and $Z_{x y}$.

$$
\begin{array}{r}
Z_{x x}=\mu_{0} \frac{<E_{x} B_{x}^{*}><B_{y} B_{y}^{*}>-<E_{x} B_{y}^{*}><B_{y} B_{x}^{*}>}{<B_{x} B_{x}^{*}><B_{y} B_{y}^{*}>-<B_{x} B_{y}^{*}><B_{y} B_{x}^{*}>} \\
Z_{x y}=\mu_{0} \frac{<E_{x} B_{y}^{*}><B_{x} B_{x}^{*}>-<E_{x} B_{x}^{*}><B_{x} B_{y}^{*}>}{<B_{x} B_{x}^{*}><B_{y} B_{y}^{*}>-<B_{x} B_{y}^{*}><B_{y} B_{x}^{*}>}
\end{array}
$$

Where noise is not random or there is excessive polarisation of the signals the above method can cause impedance estimates to be biased downwards by noise in the magnetic field but estimates are unaffected by noise in the electric field.

5. Error calculations. Variances in impedance are calculated using the method of Gamble et al. [1979a]. This method was designed for use with a remote reference site to measure the horizontal magnetic field distant to the primary sites [Gamble et al. 1979b]. For this data set, however, there was no remote reference site and consequently data errors may be underestimated.

The remaining processing steps use the impedance output from the SSMT program.

Distortion removal. The method of Bibby et al. [2005] has been used to remove distortion caused by localised, near surface heterogeneities. This method also provides some diagnostic parameters, which can be used to determine dimensionality and strike direction.

Rotation into profile co-ordinate system. MT sites were set up using magnetic north to align the magnetometers and electrodes. The impedance tensor is therefore also aligned with magnetic north $\left(\sim 21^{\circ}\right.$ east of true north). The impedance tensor can be rotated into any co-ordinate system using a rotation tensor, $\underline{\underline{R}}$.

$$
\begin{aligned}
\underline{\underline{Z}}^{\prime} & =\underline{\underline{R}} \underline{\underline{Z}} \underline{\underline{R}}^{T} \\
\underline{\underline{R}} & =\left(\begin{array}{cc}
\cos \theta & \sin \theta \\
-\sin \theta & \cos \theta
\end{array}\right)
\end{aligned}
$$

Where $\theta$ is the clockwise rotation angle from $\underline{\underline{Z}}$ to $\underline{\underline{Z}}$.

Apparent resistivity and phase. Apparent resistivity and phase are calculated for two modes from the $Z_{x y}^{\prime}$ and $Z_{y x}^{\prime}$ components of the impedance tensor using equations 4.2.15 and 4.2.16. Standard errors were calculated from the variance of the impedance.

\subsubsection{Magnetotelluric results}

The following results are plotted against the $\log _{10}$ of the period, $T$, where $T=2 \pi / \omega$. Using this convention depth of penetration increases with $T$. 


\section{Phase tensor analysis and dimensionality}

The phase tensor method used to remove distortion [Bibby et al. 2005] also outputs a number of parameters that can be used to determine the dimensionality of the electrical structure and indicate the approximate 2D strike direction. Figures 4.7-4.12 show the results of this analysis for each station. For each station three analysis quantities are shown. The first graph shows the variation in phase ellipticity, $\lambda$, with $T$ and is a measure of how close the data are to a $1 \mathrm{D}$ structure. For $\lambda \leqslant 0.15$ the structure can be considered to be 1D. The second graph gives a measure of the departure from simple $2 \mathrm{D}$ symmetry. Data can be considered to be $2 \mathrm{D}$ if $\lambda>0.15$ and $\beta$ is small $\left(<5^{\circ}\right)$. The larger the value of $\beta$ the larger the departure from the simple 2D case [Bibby et al. 2005]. The last graph gives the principal direction of the phase tensor $(\alpha-\beta)$. The principal direction of the phase tensor can be used to determine the strike direction of the resistivity structure, however there is a 90 degree ambiguity in this measure since this principal direction can be either perpendicular or parallel to the strike direction.

For all stations, short period data have $\lambda<0.15$ indicating that the shallow structure is essentially 1D. The $T$ at which $\lambda$ first exceeds 0.15 varies from station to station. With the exception of stations 002 and 006 the azimuth of the phase tensor has two clear directions. A shorter period direction of approximately $-30^{\circ}$ to magnetic north and a longer period direction of approximately $\pm 80^{\circ}$ to magnetic north. Station 002 (figure 4.8) is $1 \mathrm{D}$ to longer periods than the other stations and the $2 \mathrm{D}$ data has only the $\pm 80^{\circ}$ azimuth. Station 006 (figure 4.12 ) also has the long period $\pm 80^{\circ}$ azimuth, but has an azimuth that varies from $-45^{\circ}$ to $-90^{\circ}$ from $T=20 \mathrm{~s}$ to $T=170 \mathrm{~s}$. For all stations, errors for all three parameters increase beyond $T=3600 \mathrm{~s}$ and become scattered.

Figure 4.13 shows three rose diagrams of the phase tensor principal directions relative to true north for $\lambda>0.15$ and $\beta<5$. The first shows the principal directions of all six stations and the other two divide the results into 3 northern stations (001-003) and 3 southern stations. The two strike directions from figures $4.7-4.12$ can be seen clearly in these plots, the first is between $330^{\circ}$ and $360^{\circ}$ (shown in red) and the second between $90^{\circ}$ and $120^{\circ}$ (shown in blue). For the northern 3 stations the long period principal direction is in the azimuth range $90^{\circ}-125^{\circ}$. For the southern stations the long period principal direction is in the range $295^{\circ}-300^{\circ}$.

Because there is a $90^{\circ}$ ambiguity in correlating these principal directions with the geoelectric strike, additional information is required. The short period principal direction corresponds to crustal depths $(1-35 \mathrm{~km})$. The $-15^{\circ}$ azimuth is not parallel or perpendicular to any prevalent structural features such as faulting or basement terranes (figure 4.5). However ellipticity of the phase tensor may also be caused by a change from isotropic resistivity to anisotropic resistivity [Heise et al. 2006]. Shallow seismic 


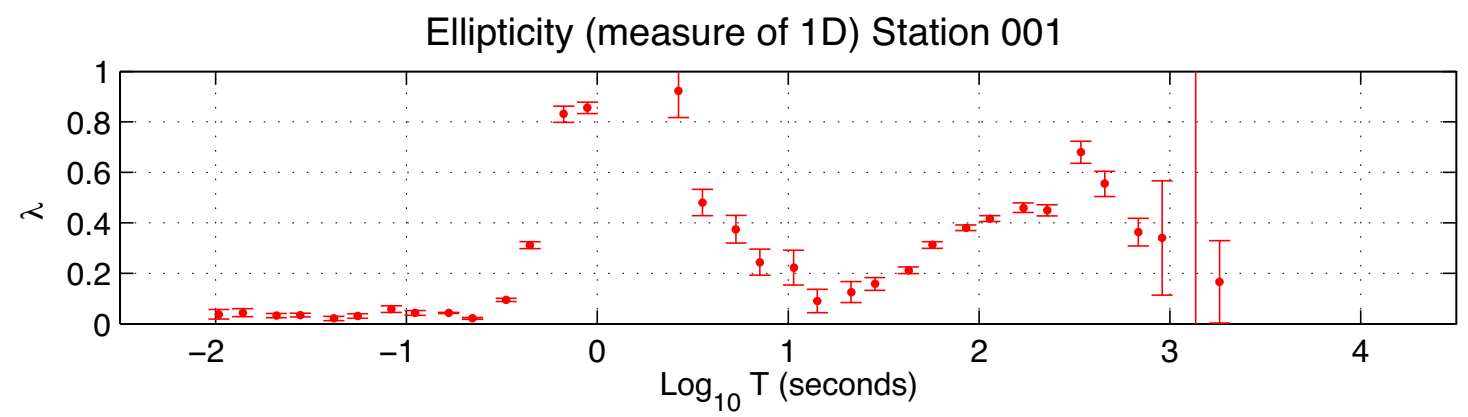

Phase tensor $\beta-2 D$ Station 001

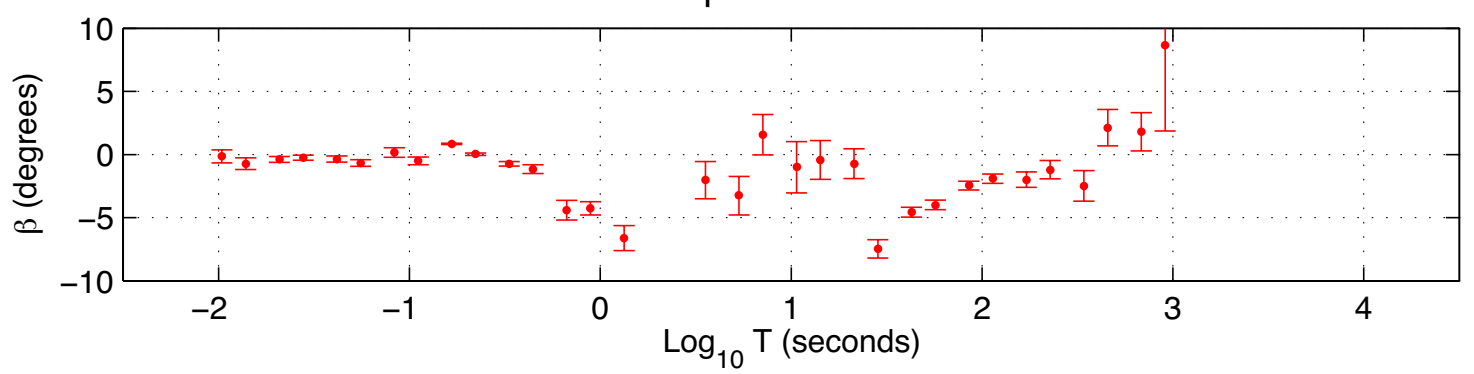

Azimuth of Phase tensor maximum Station 001

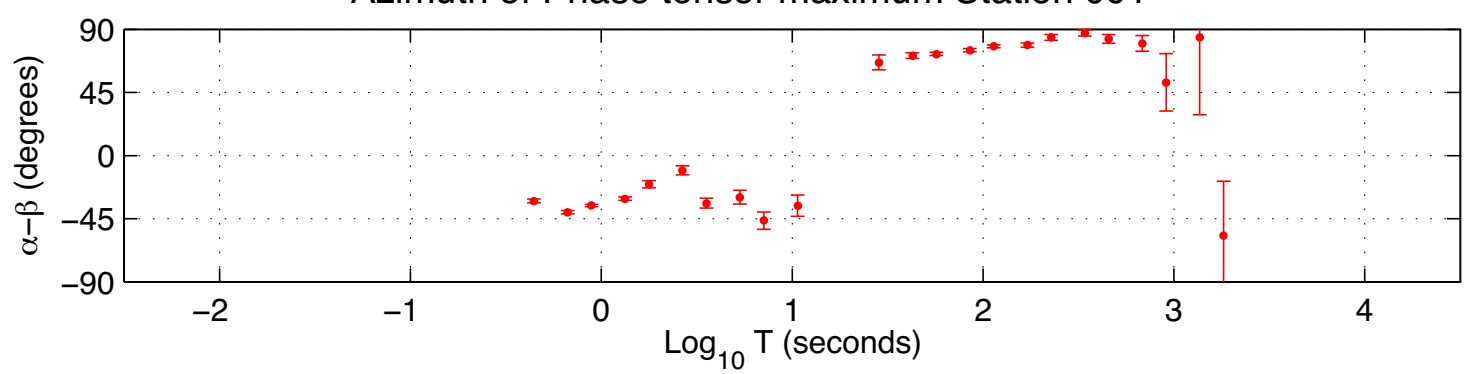

Figure 4.7: Phase tensor results for station 001. 
Ellipticity (measure of 1D) Station 002

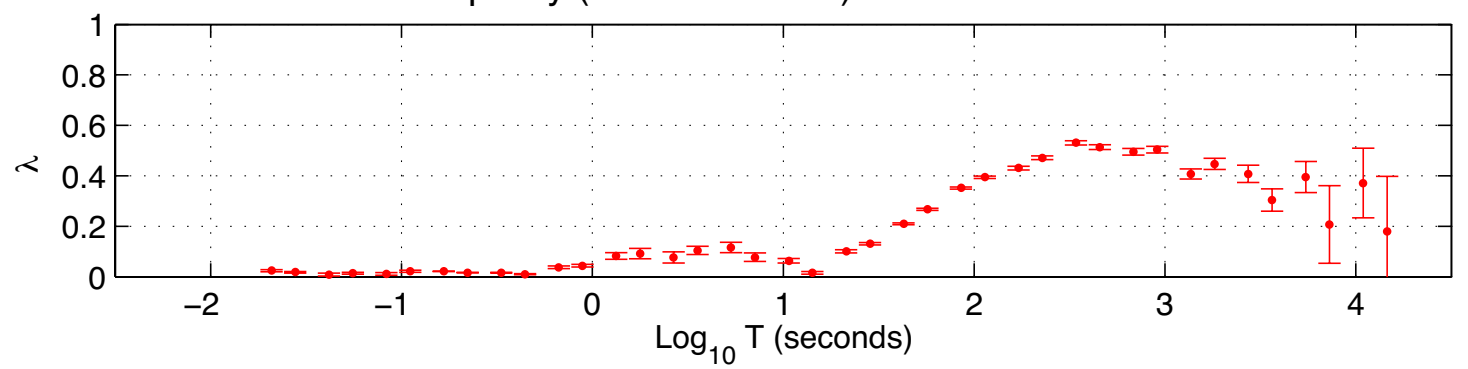

Phase tensor $\beta-2 D$ Station 002

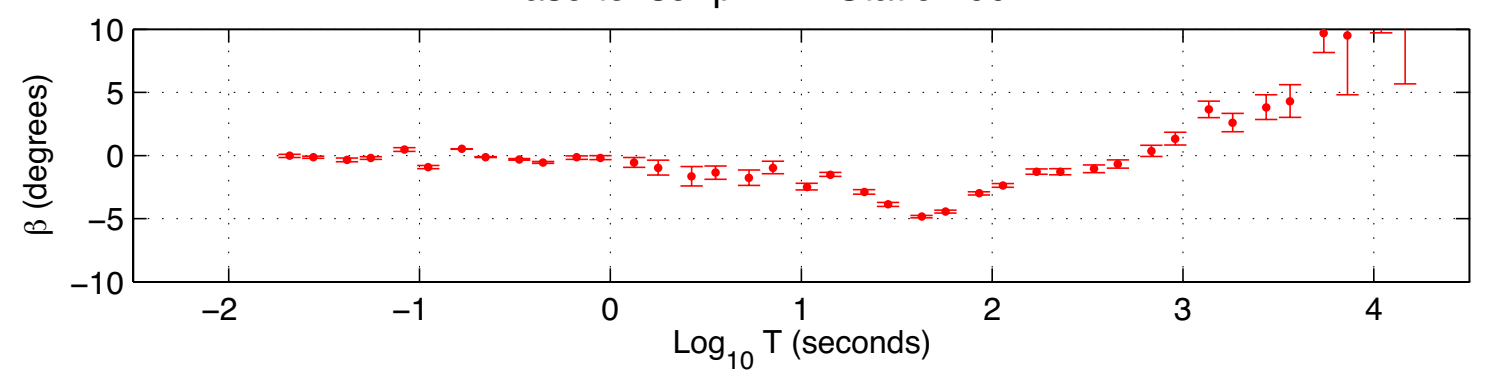

Azimuth of Phase tensor maximum Station 002

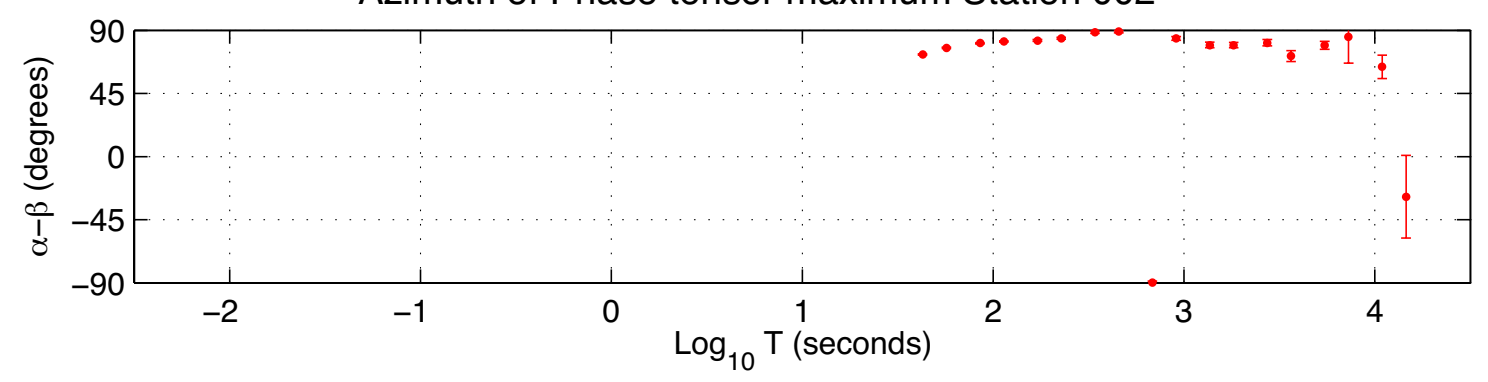

Figure 4.8: Phase tensor results for station 002 
Ellipticity (measure of 1D) Station 003

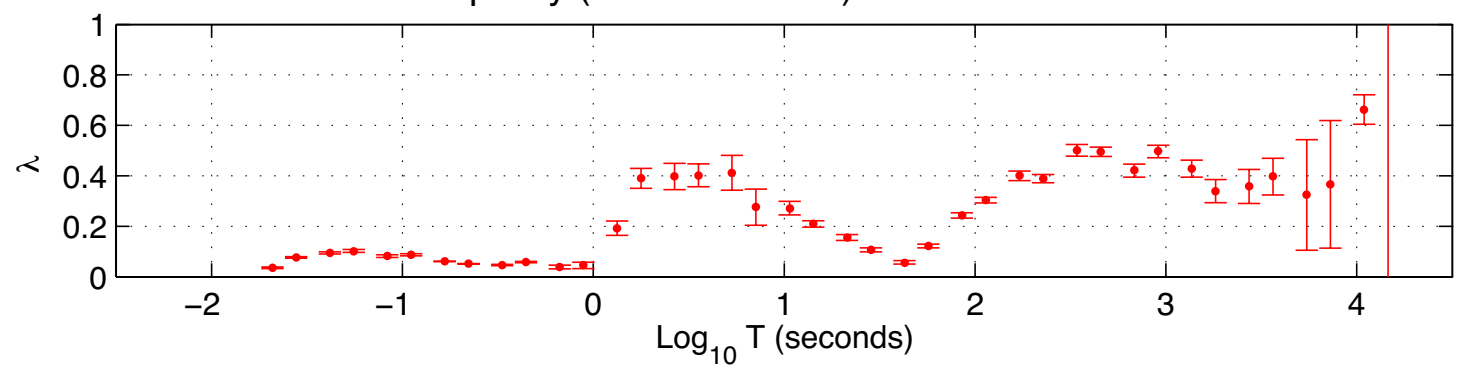

Phase tensor $\beta-2 \mathrm{D}$ Station 003

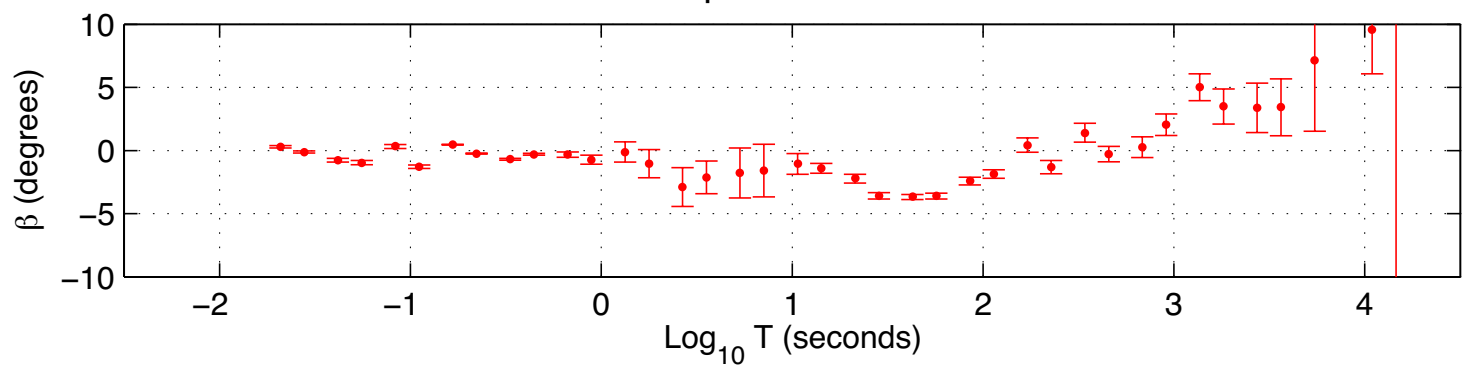

Azimuth of Phase tensor maximum Station 003

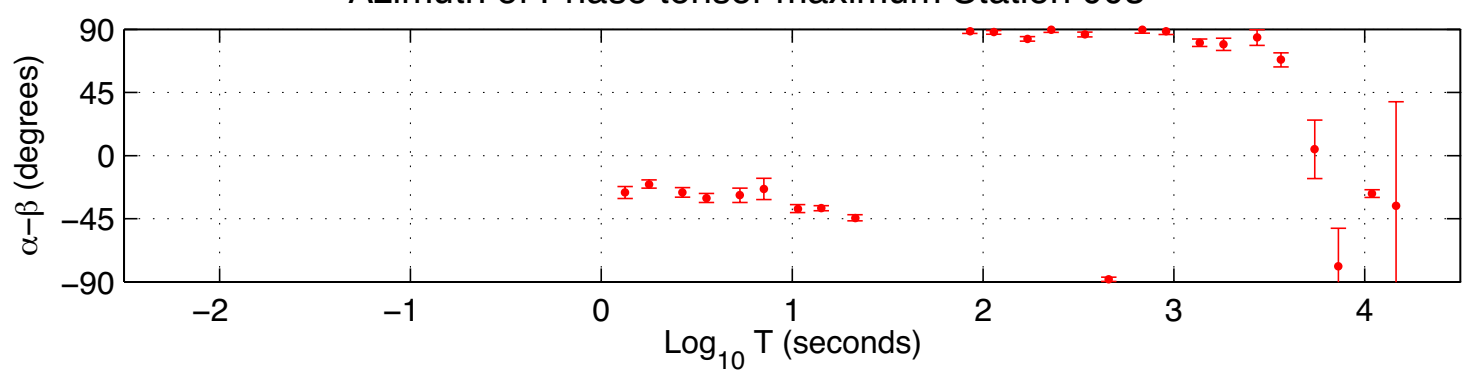

Figure 4.9: Phase tensor results for station 003 
Ellipticity (measure of 1D) Station 004

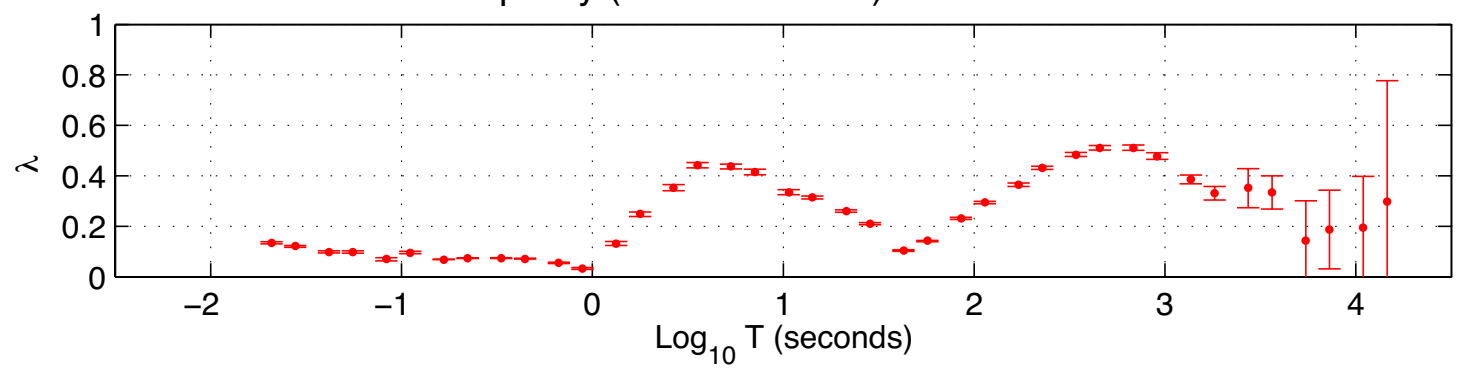

Phase tensor $\beta-2 D$ Station 004

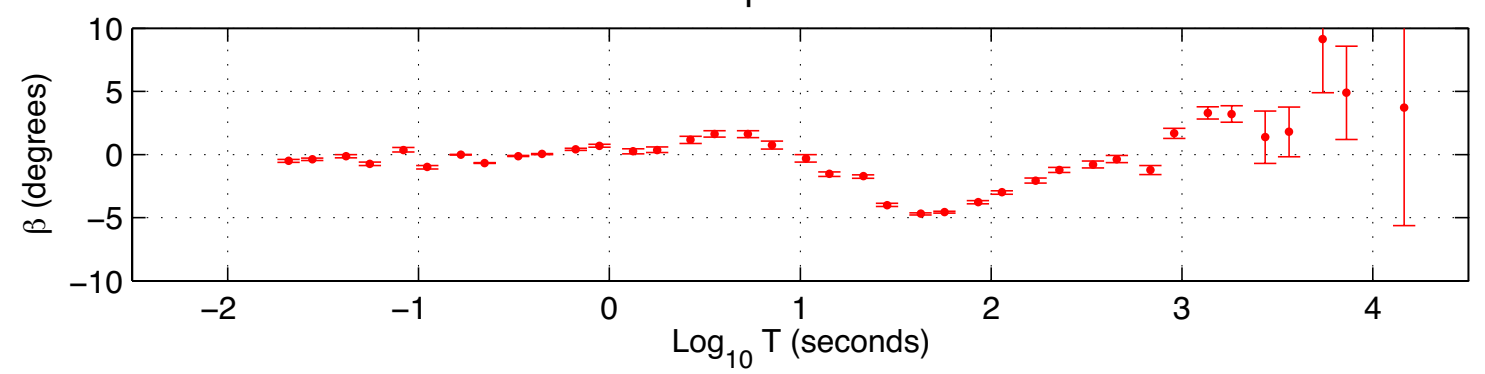

Azimuth of Phase tensor maximum Station 004

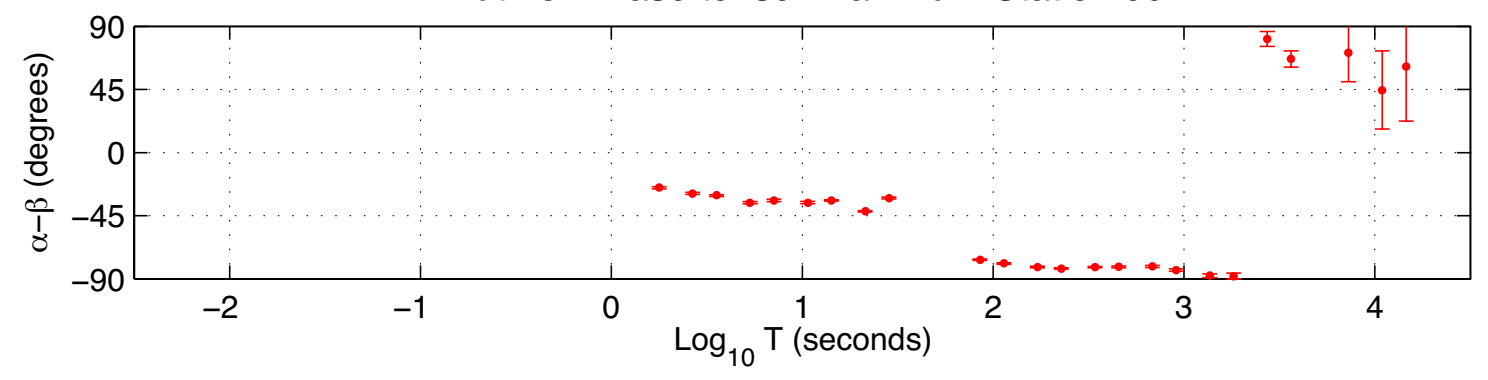

Figure 4.10: Phase tensor results for station 004 
Ellipticity (measure of 1D) Station 005

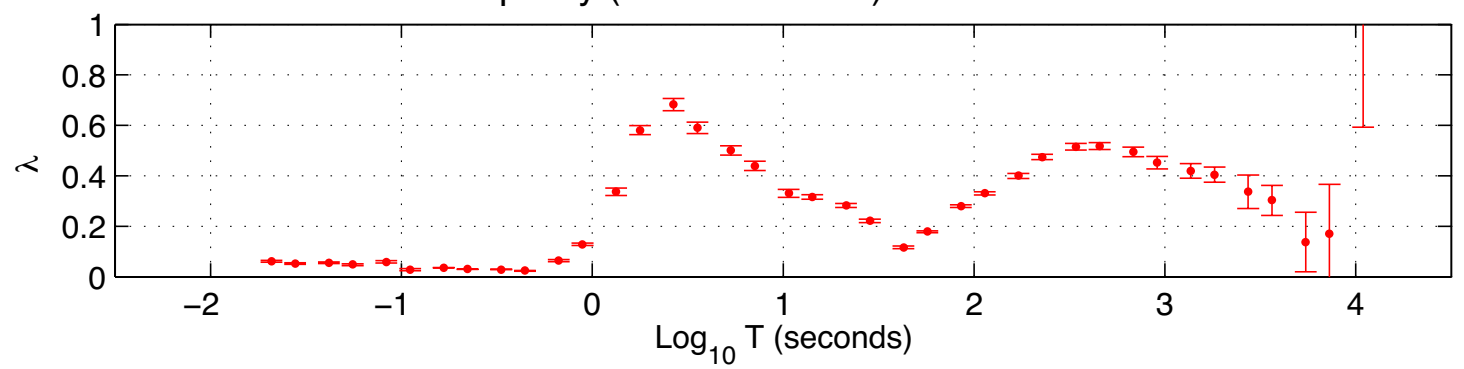

Phase tensor $\beta-2 D$ Station 005

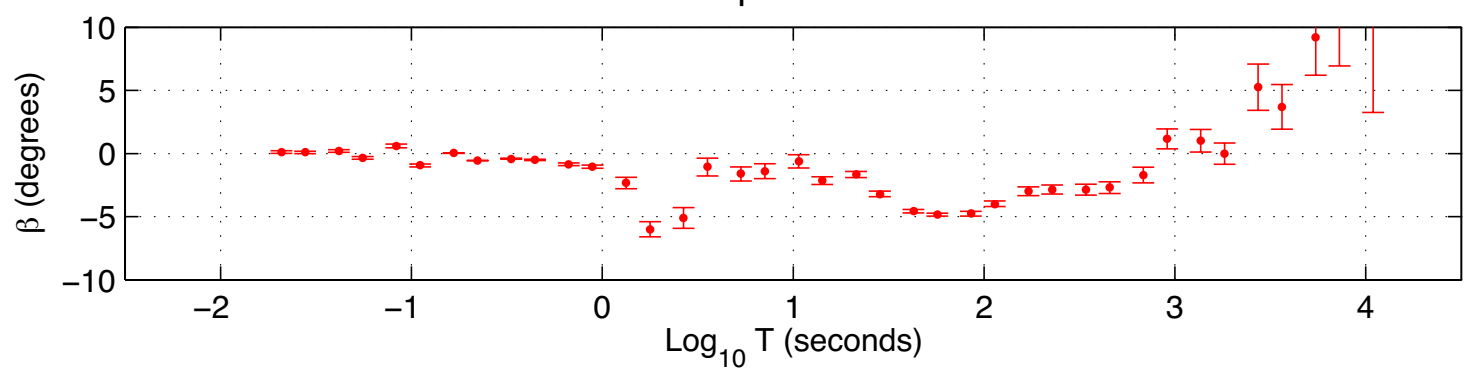

Azimuth of Phase tensor maximum Station 005

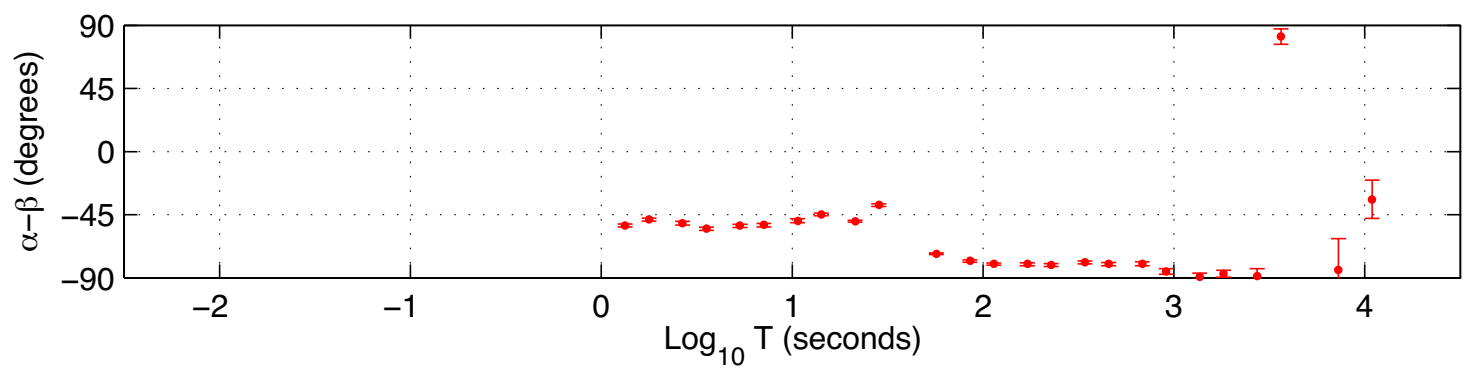

Figure 4.11: Phase tensor results for station 005 
Ellipticity (measure of 1D) Station 006

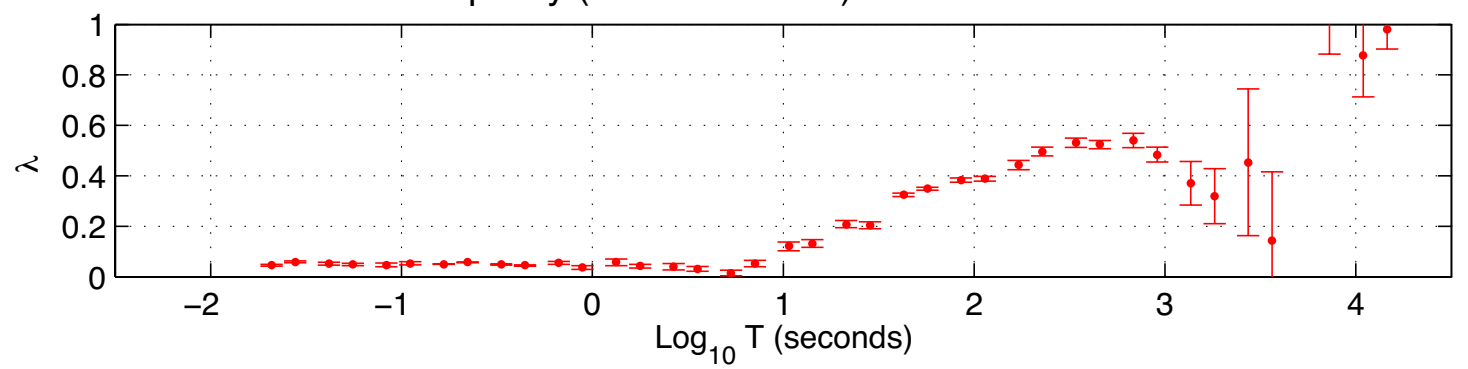

Phase tensor $\beta-2 D$ Station 006

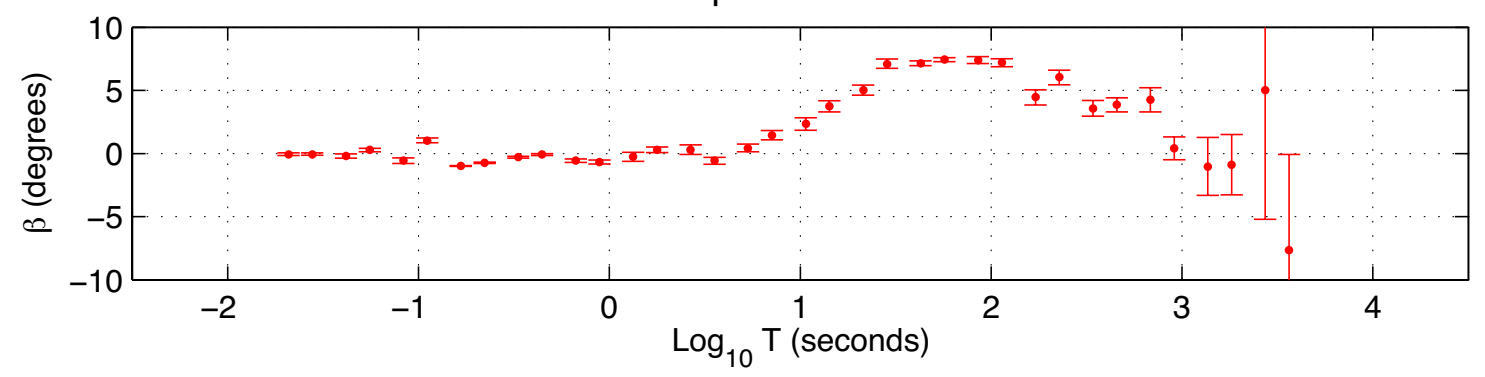

Azimuth of Phase tensor maximum Station 006

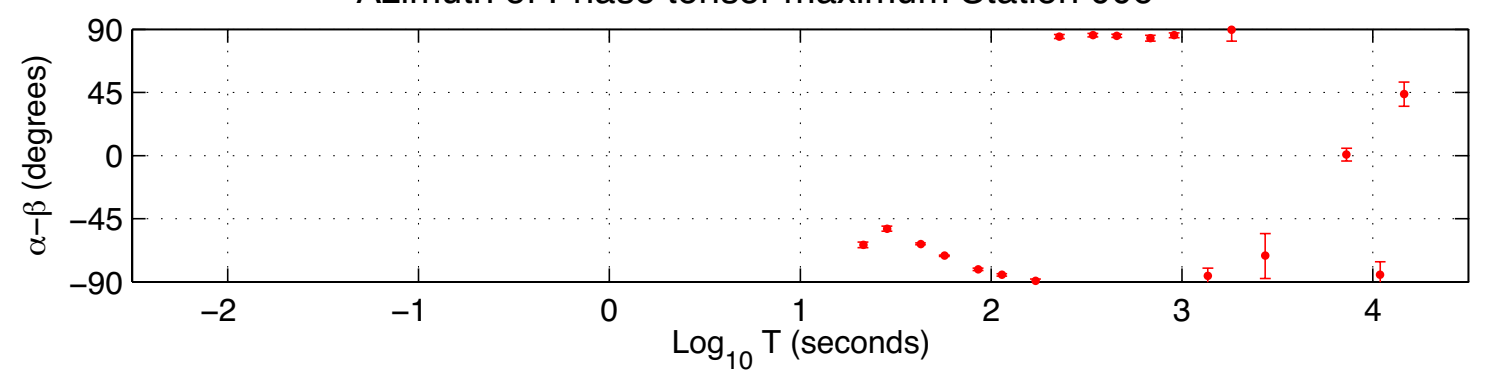

Figure 4.12: Phase tensor results for station 006 

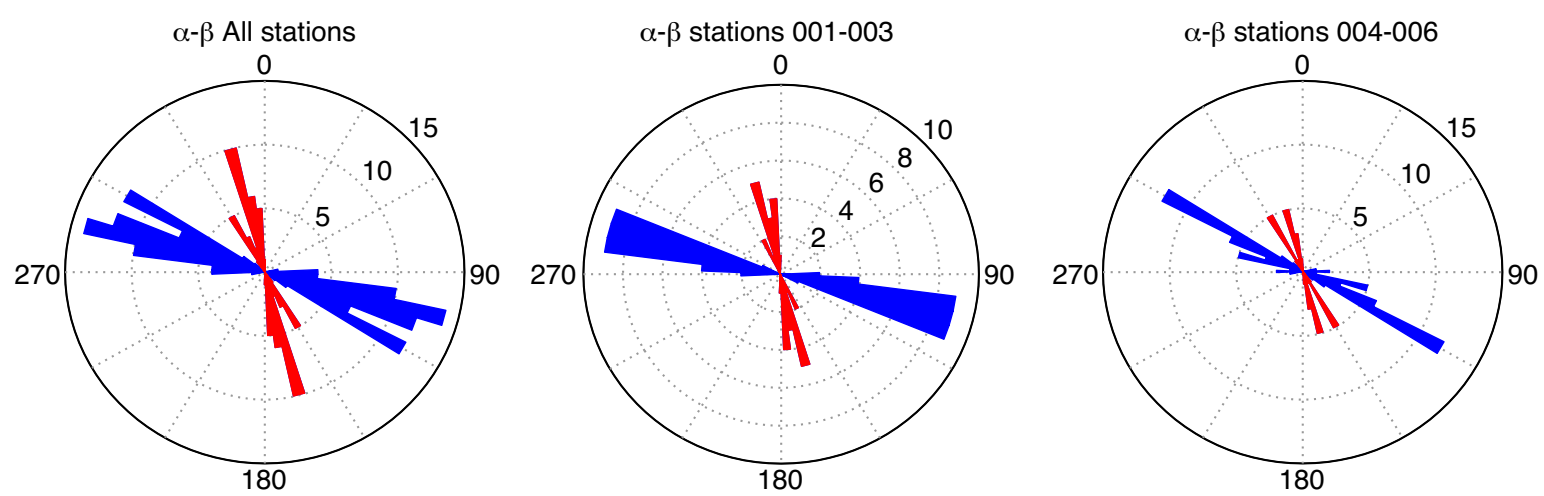

Figure 4.13: Orientation of the phase ellipse for $\beta<5$ oriented to true north. The rose diagrams plot the number of phase ellipses oriented in each direction over the period range where $\lambda>0.15$ and $\beta<5$. The red sectors are for the shorter period data and the blue is the longer period data. The first rose diagram shows all stations together. The second is for the northern stations and the last is for the southern stations. It should be noted that station 005 has the highest average angle of 118 degrees.

anisotropy is observed close to the MT line indicating the presence of an anisotropic crustal layer [Audoine et al. 2000; 2004].

The longer period principal directions correspond to crust/upper-mantle depths $(>26$ $\mathrm{km})$. For the northern 3 sites this azimuth $\left(100 \pm 10^{\circ}\right)$ is approximately parallel to isostatic gravity anomaly contours in the region (figure 1.2) and perpendicular to local strike of basement terranes and faulting. The southern sites have a long period principal direction closer to $120^{\circ}$, approximately perpendicular to the regional tectonic strike of the Hikurangi subduction zone. As seen previously (chapter 1.2) the steep gradient in the gravity anomaly can be modelled as a change in crustal thickness and hence fits with a geo-electric strike direction that is approximately E-W. Assuming an E-W geo-electric strike means that the $x y$ and $y x$ components of the phase and apparent resistivity correspond to the TM and TE mode respectively.

\section{Apparent resistivity and phase}

Figures 4.14 to 4.19 show apparent resistivity and phase data with distortion corrections rotated into the geo-electric strike of the northern sites ( $10^{\circ}$ clockwise from true north). The TM mode is represented by $\rho_{x y}$ and $\phi_{x y}$ and the TE mode is represented by $\rho_{y x}$ and $\phi_{y x}$. The error bars indicate standard errors. Data quality can be gauged qualitatively by both the size of the error bars and the smoothness of the curve. Since the propagation of electromagnetic waves can be described by the diffusion equation, both apparent resistivity and phase curves should be relatively smooth. Station 001 had the shortest recording time and hence this station has the poorest data quality. At $T$ between 0.5 and $5 \mathrm{~s}$, errors tend to increase and data curves become less smooth. This range of data is in what is often referred to as the dead band [e.g. Simpson and 
Bahr 2005, pg. 3], a frequency range where there is a drop in signal strength. With the exception of stations 001 and 006 data quality is generally good out to $\sim 3500 \mathrm{~s}$.

\section{Short period apparent resistivity and phase}

At short periods $\rho_{x y}$ and $\rho_{y x}$ are concurrent indicating an approximately 1D shallow structure. With the exception of station 005 the phase and apparent resistivity at short periods follows a similar pattern for all stations. Apparent resistivity initially decreases with increasing $T$. This decrease in resistivity is matched by an increasing phase angle. The $T$ at which the minimum apparent resistivity is reached increases southward from $0.22 \mathrm{~s}$ at station 001 in the north (figure 4.14) to $5.3 \mathrm{~s}$ at station 006 in the south (figure 4.19). Apparent resistivities at the shortest periods also increase to the south from $10 \Omega \mathrm{m}$ at station 001 to $40 \Omega \mathrm{m}$ at station 006 . These resistivities are within the range expected for the sediments of the Wanganui Basin [Ingham et al. 2001]. Using these resistivities and equation 4.2.11 to estimate the minimum penetration depth, the shortest period (0.021 s) samples the top 160-480 m (appendix D table D.1).

For station 005 there is no initial decrease in the apparent resistivity, the resistivity instead remains nearly constant for $T<1.0 \mathrm{~s}$ at $\sim 5 \Omega \mathrm{m}$. This resistivity is less than that seen at the other stations. The phase at this station has no initial increase, instead the phase at $0.021 \mathrm{~s}$ is initially above $45^{\circ}$ and decreases with increasing $T$. This station was located in a boggy field close to a small stream. Since the presence of interconnected water enhances conductivity, an elevated water table could account for the low resistivity at this site. This offset reduces the expected basement depth based on the contour plot (see figure 4.5). This site is also offset to the east of the other stations, which may be a contributing factor to the differences seen between the resistivity and phase curves of this station and the remainder of the sites.

Stations 001 to 005 initially have a phase above $45^{\circ}$ indicating that there is a higher resistivity surface layer, which is thinner than the skin depth of the shortest period. At station 006 the phase is initially just below $45^{\circ}$ indicating that there is a low resistivity layer at the surface, which is thinner than the penetration depth of the shortest period.

Short period observations are consistent with a sedimentary layer, which increases in resistivity with depth overlying a low resistivity sedimentary layer. Sediments of the Wanganui Basin thicken along the MT line. At the northern end the sediments are thinner and the low resistivity layer is closer to the surface. At the southern end the sediments are thicker than the minimum skin depth.

\section{Long period resistivity and phase}

At longer periods the $\rho_{x y}$ and $\rho_{y x}$ curves diverge. Beyond the low in resistivity at short periods both modes have increasing resistivity with increasing $T$. For $T \gtrsim 40 \mathrm{~s} \rho_{x y}$ 
flattens out while the $\rho_{y x}$ continues to increase to periods of at least $1000 \mathrm{~s}$. The phase curves also diverge at longer periods. A minimum in $\phi_{x y}$ is observed between periods of $2 \mathrm{~s}$ and $30 \mathrm{~s}$. Again, with the exception of station 005, the $T$ at which this minimum occurs increases to the south. Using equation 4.2.11 the depth at which this minimum in $\phi_{x y}$ occurs is roughly from 4 to $16 \mathrm{~km} . \phi_{x y}$ and $\phi_{y x}$ are nearly coincident for all stations to at least this minimum in $\phi_{x y}$. With the exception of station $006 \phi_{x y}$ and $\phi_{y x}$ then increase together until periods of $\sim 30$ s then $\phi_{y x}$ decreases again reaching a local minimum between 220 and $350 \mathrm{~s}$. At station 006 the two curves diverge where $\phi_{x y}$ reaches its minimum $(T=30 \mathrm{~s})$. For this station the $\phi_{y x}$ curve continues to decrease to $T=230 \mathrm{~s}$. The skin depth of the kink in the $\phi_{y x}$ curve is $\sim 20-30 \mathrm{~km}$.

\section{Pseudo-sections}

Pseudo-sections are used to illustrate the changes in phase and apparent resistivity along a profile perpendicular to the structure of interest. Figures 4.20 and 4.21 are contour plots of the data shown in figures 4.14 to 4.19 plotted along a profile with an azimuth of $10^{\circ}$. The $x y(\mathrm{TM})$ and $y x$ (TE) modes are shown separately.

Apparent resistivities vary considerably along the profile. Some of this variation may be due to static shifts, which are not accounted for when using the phase tensors to remove distortion. The pseudo-sections allow the comparison of lateral changes in the phase and resistivity. Since the phase is unaffected by static shift, if significant lateral changes in resistivity are accompanied by lateral changes in the phase they can be assumed to be real. Where this is not the case static shift may be a contributing factor.

The apparent resistivity at station 005 is anomalous at short periods. Below this station short period data have a lower resistivity than the adjacent stations. This lateral change in resistivity is reflected in a change in phase at this station. It can therefore be assumed that the change in short period resistivity at this station is real. This station is offset to the east of the other stations and may be sampling different structures. For the remainder of the stations, pseudo-sections confirm the $T$ at which the minimum resistivity is reached generally increases to the south. At longer periods the phase data are relatively smooth. For the $x y$ mode the contours slope to the south. The resistivity contours of both modes oscillate up and down. This may in part be due to static shifts.

\subsection{MT modelling}

Although some knowledge of the subsurface resistivity can be gained from apparent resistivity and phase curves, the ultimate goal is to determine a $2 \mathrm{D}$ resistivity model. Phase tensor analysis shows that the short period data are approximately 1D and 

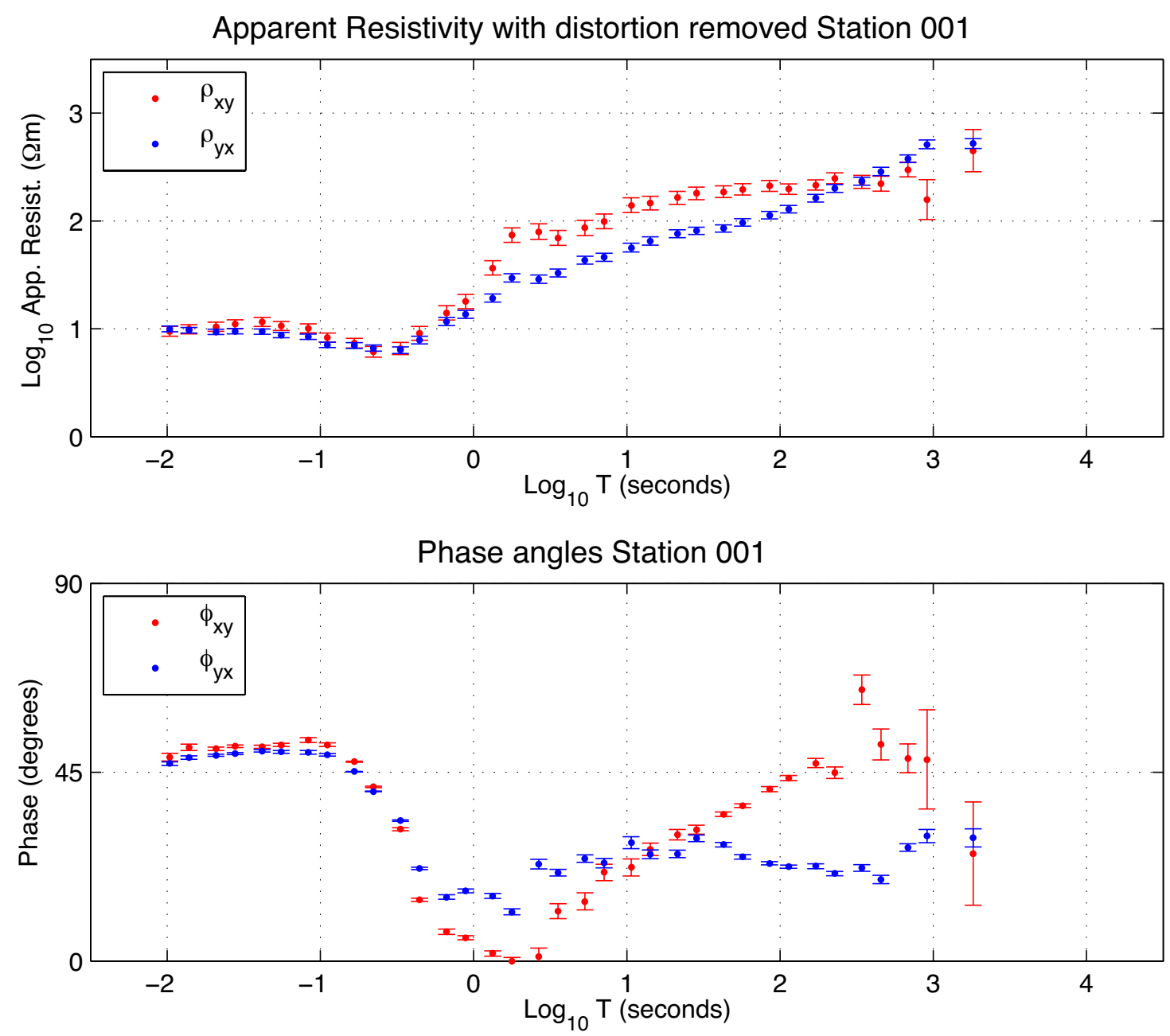

Figure 4.14: Resistivity and phase results for station 001. Data have been rotated so that the principal directions are $10^{\circ}$ and $100^{\circ}$ from true north. Calculated standard errors are shown as error bars. The TM mode data are shown in red and the TE mode is shown in blue. 

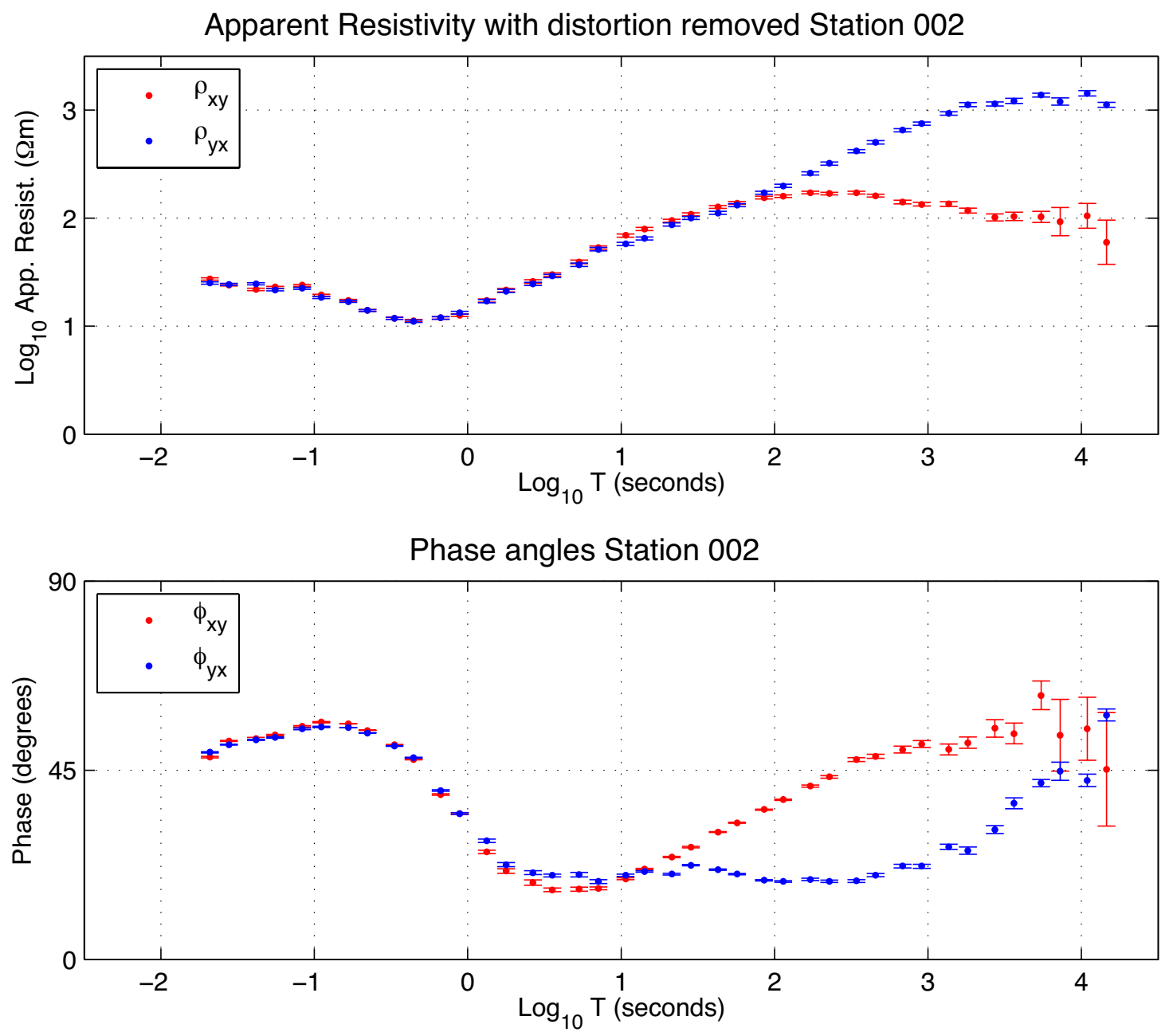

Figure 4.15: Resistivity and phase results for station 002. Data have been rotated so that the principal directions are $10^{\circ}$ and $100^{\circ}$ from true north. Calculated standard errors are shown as error bars. The TM mode data are shown in red and the TE mode is shown in blue. 


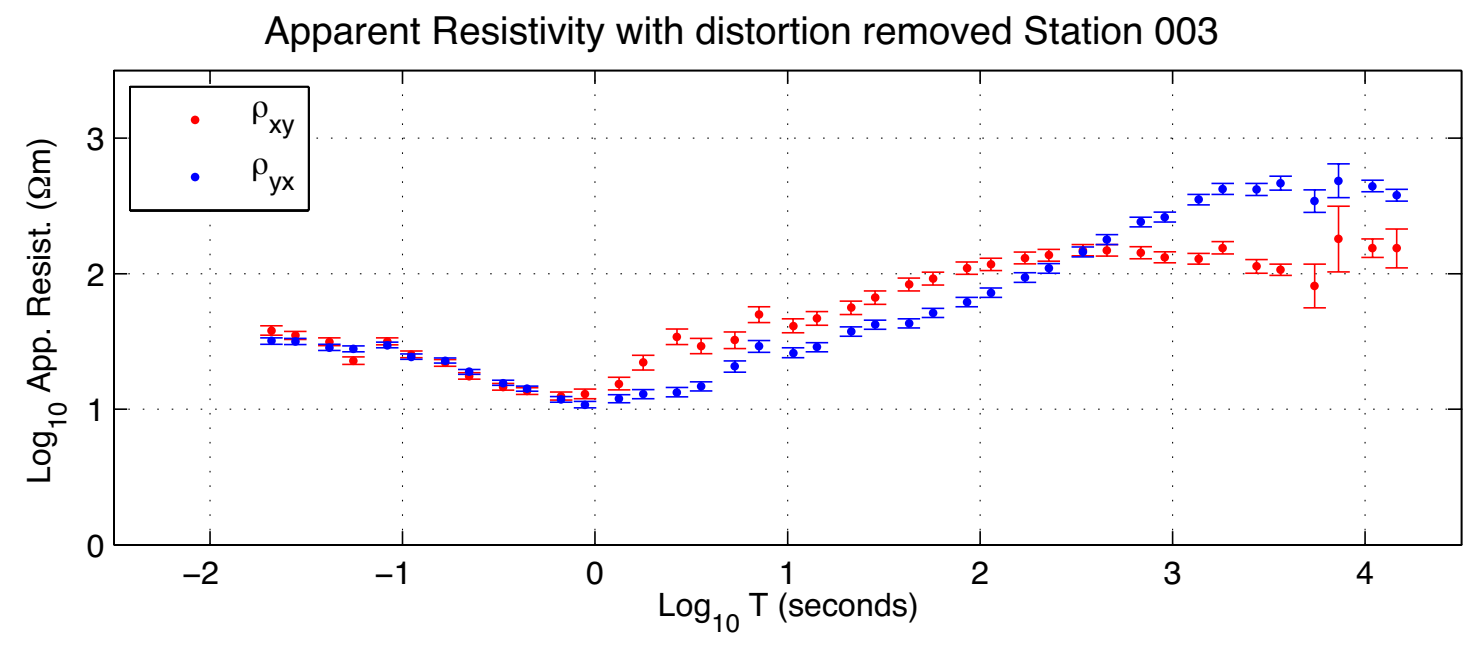

Phase angles Station 003

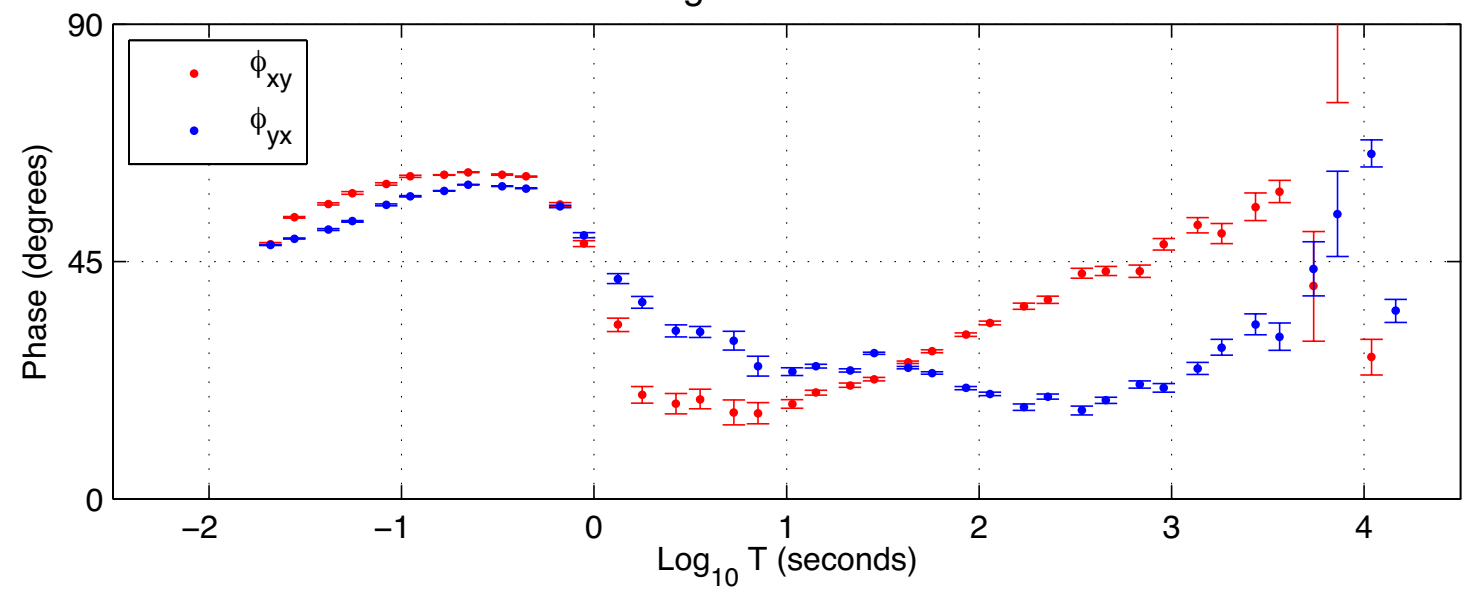

Figure 4.16: Resistivity and phase results for station 003. Data have been rotated so that the principal directions are $10^{\circ}$ and $100^{\circ}$ from true north. Calculated standard errors are shown as error bars. The TM mode data are shown in red and the TE mode is shown in blue. 

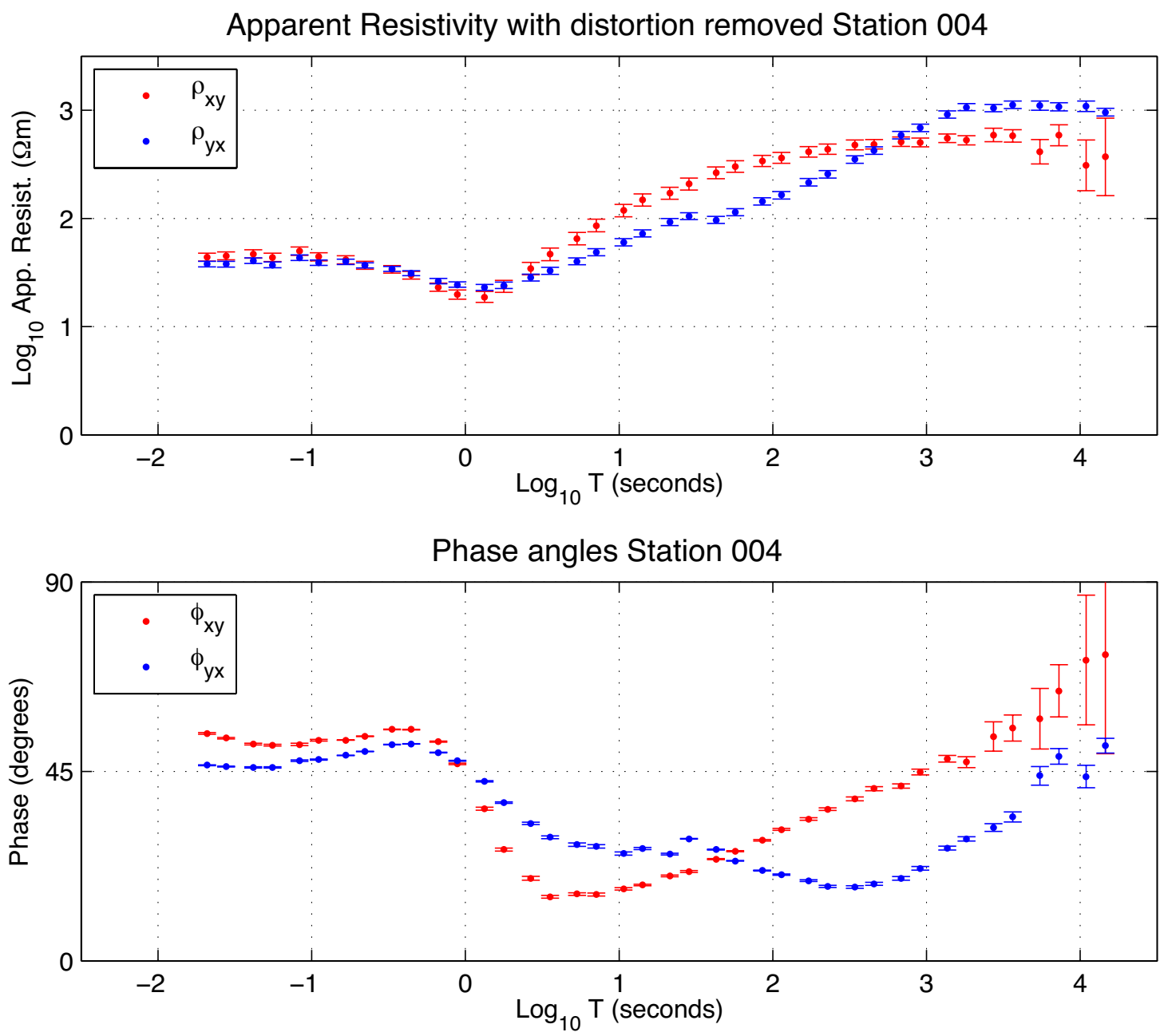

Figure 4.17: Resistivity and phase results for station 004. Data have been rotated so that the principal directions are $10^{\circ}$ and $100^{\circ}$ from true north. Calculated standard errors are shown as error bars. The TM mode data are shown in red and the TE mode is shown in blue. 

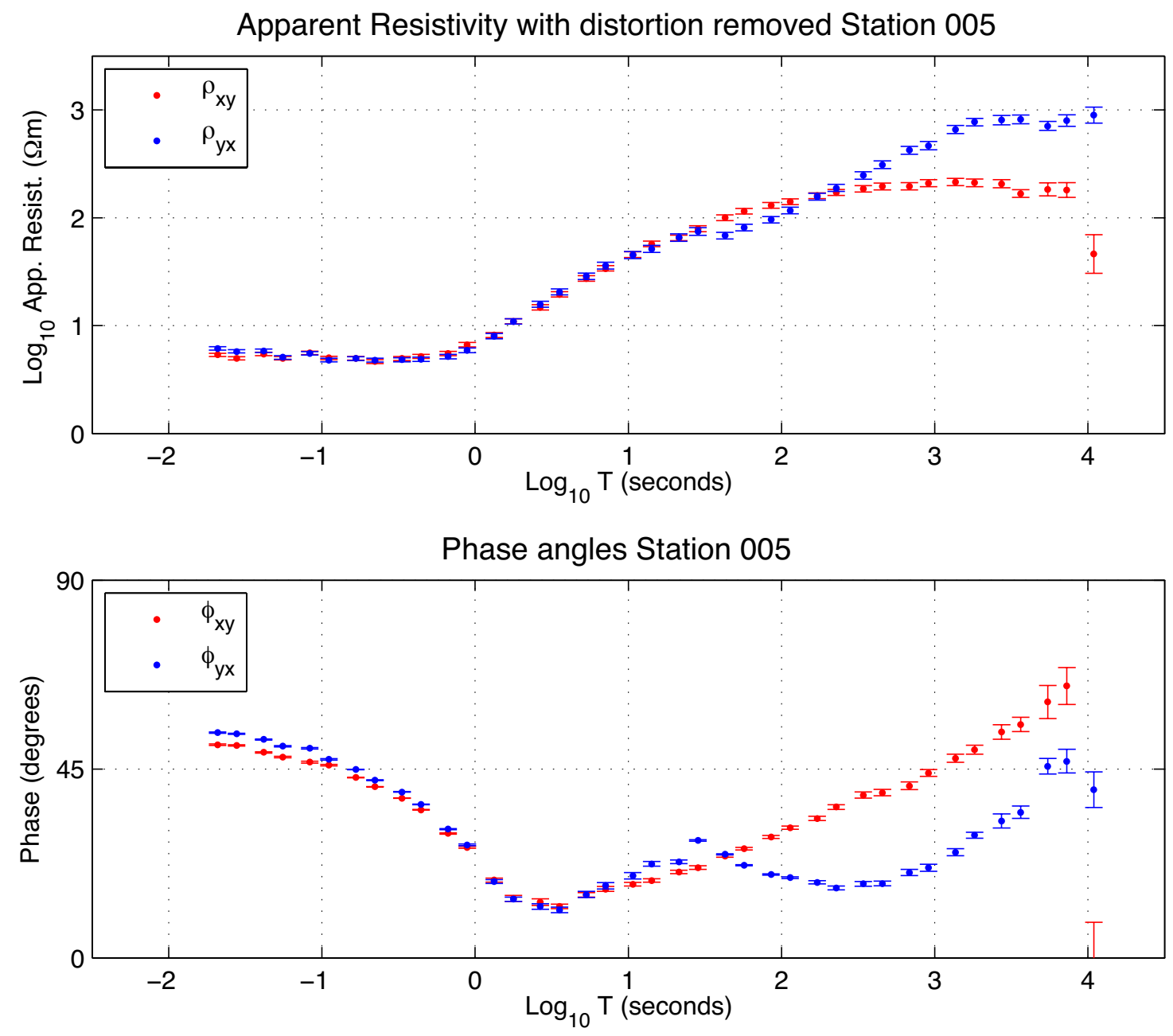

Figure 4.18: Resistivity and phase results for station 005. Data have been rotated so that the principal directions are $10^{\circ}$ and $100^{\circ}$ from true north. Calculated standard errors are shown as error bars. The TM mode data are shown in red and the TE mode is shown in blue. 

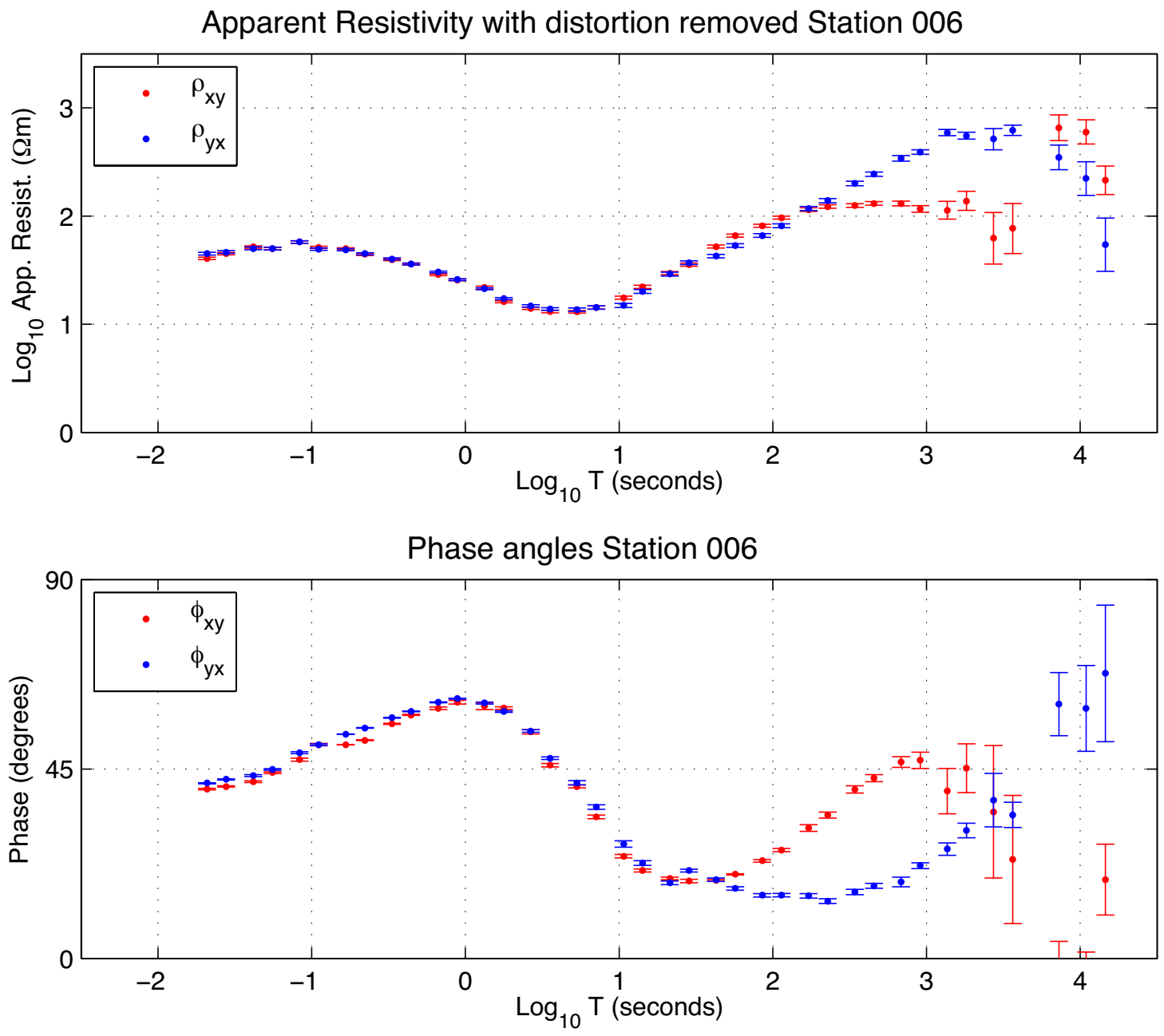

Figure 4.19: Resistivity and phase results for station 006. Data have been rotated so that the principal directions are $10^{\circ}$ and $100^{\circ}$ from true north. Calculated standard errors are shown as error bars. The TM mode data are shown in red and the TE mode is shown in blue. 

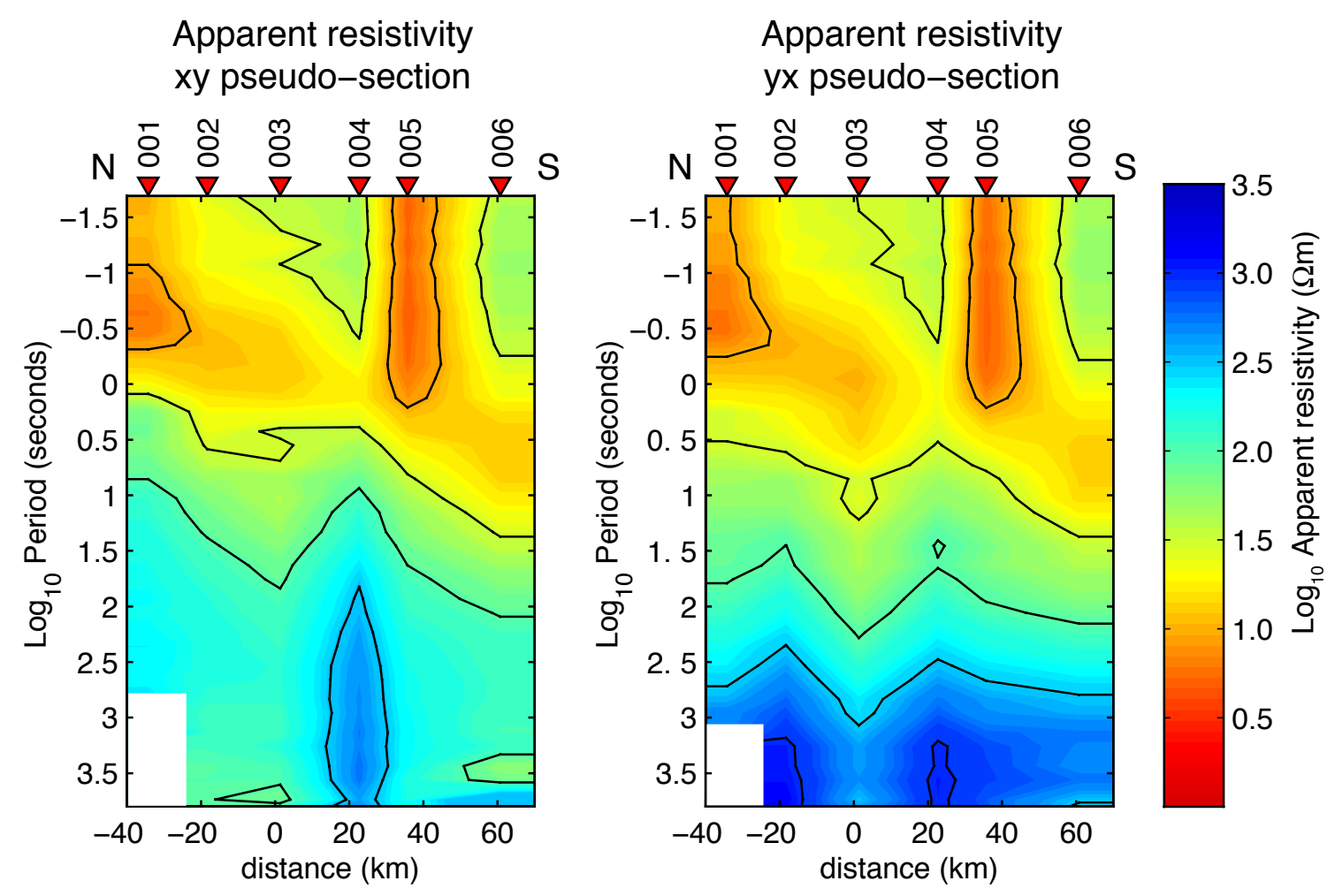

Figure 4.20: Apparent resistivity pseudo-sections. TM mode (xy) on the left and TE mode $(y x)$ on the right. Black contours are at $10^{0.5} \Omega \mathrm{m}$ intervals. Low apparent resistivities are shown in oranges and yellows and high resistivities are shown in blue. Distances are measured from the TRL. Apart from the general increase in resistivity with $T$, the most notable feature is the decrease in short period resistivity at station 005. Principal directions are $10^{\circ}$ and $100^{\circ}$ from true north. 

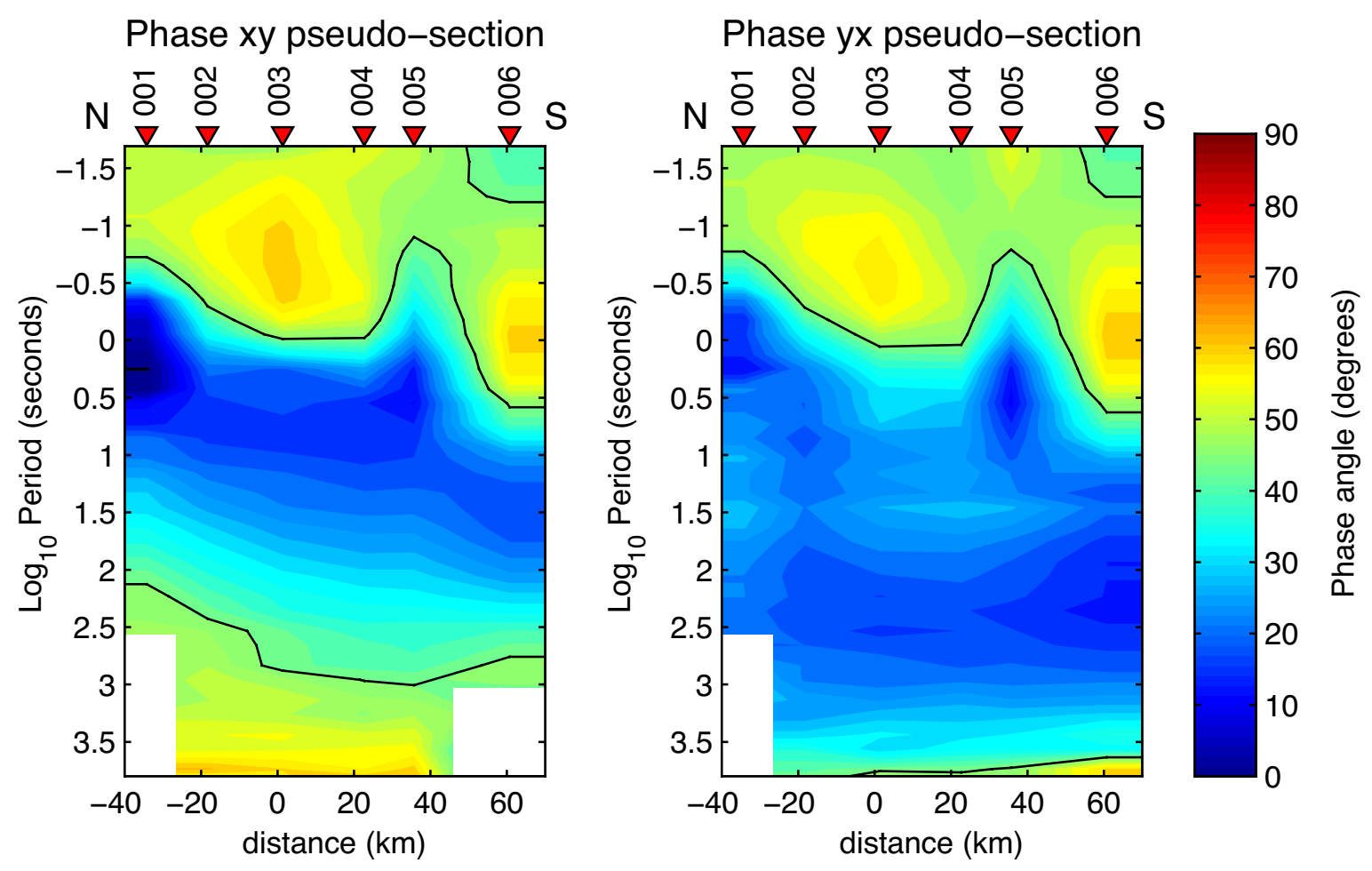

Figure 4.21: Phase pseudo-sections. TM mode $(x y)$ on the left and TE mode $(y x)$ on the right. The black contour lines are at a phase angle of $45^{\circ}$, which marks the change from decreasing resistivity with depth $\left(\phi>45^{\circ}\right)$ to increasing resistivity with depth $\left(\phi<45^{\circ}\right)$. Distances are measured from the TRL. In comparison to the apparent resistivity, phase varies relatively smoothly across the stations. The feature that stands out most is change in short period phase below station 005. Principal directions are $10^{\circ}$ and $100^{\circ}$ from true north. 
therefore the modelling process begins with a 1D inversion.

\subsubsection{D modelling}

For a 1D earth it is theoretically possible to determine a unique resistivity model analytically, however this assumes continuous noiseless data. In reality noise is always present and data are never continuous. This introduces a degree of non-uniqueness into any solution. Data were inverted using the Occam's inversion technique [Constable et al. 1987]. This inversion scheme attempts to meet the minimum fit requirements with the smoothest possible model. In order to minimise the complexity of the model structure, a roughness parameter, $R$, is defined as the integrated square of the first derivative with respect to depth:

$$
R=\int(d m / d z)^{2} d z
$$

where $m(z)$ is the log of the resistivity.

Occam's inversion minimises the roughness parameter for the misfit, thus returning the smoothest possible model. Since the magnetotelluric method is diffusive and cannot resolve sharp boundaries, the Occam's inversion scheme fits the nature of the data. This inversion scheme also has the advantage that it is independent of the starting model. Since the Occam's inversion returns the smoothest possible structure, the true structure must be at least as complex as the model returned.

An Occam's inversion was performed using the MT sounding module of the WinGLink proprietary software [GEOSYSTEM SRL 2005]. The forward model uses equation 4.2.19 to determine the surface impedance of a layered model at each frequency. Equations 4.2.15 and 4.2.16 are then used to determine apparent resistivity and phase. Minimum and maximum layer depths for each station were estimated from the minimum and maximum skin depths (appendix D table D.1). The initial model consisted of 20 layers all with $100 \Omega$ m resistivity. Layer thickness increases logarithmically with depth. The TM mode apparent resistivity and phase data were used for the 1D inversion since this mode is more sensitive to resistivity structure than the TE mode [deGroot-Hedlin and Constable 1990]. Static shifts were assumed to be zero and the apparent resistivity and phase were jointly inverted. 1D resistivity models and data fits are shown in figures 4.22 and 4.23 .

\section{D modelling results}

At shallow depths $(<10 \mathrm{~km})$ models at all stations follow a similar pattern, a low resistivity region $(<300 \Omega \mathrm{m})$ over higher resistivity $(>300 \Omega \mathrm{m})$. The low resistivity region has a minimum resistivity of approximately $10 \Omega \mathrm{m}$. The depth at which resis- 

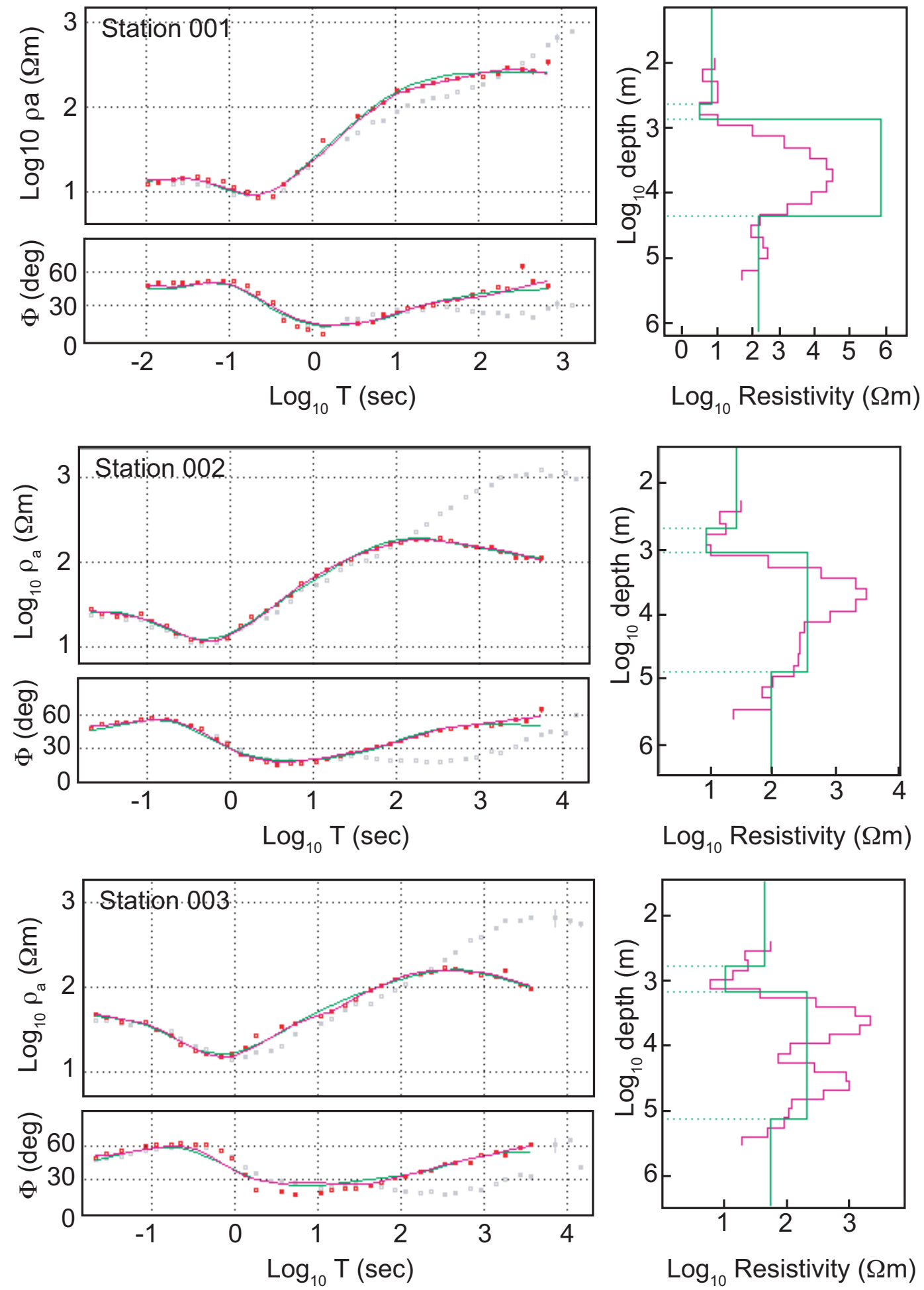

Figure 4.22: 1D models for stations 001-003. On the left TM mode apparent resistivity and phase data are shown as red dots and the TE data are shown in grey. The pink line is fit to the data for the 1D Occam model also in pink on the left. The green line is for a 4-layer resistivity model. 

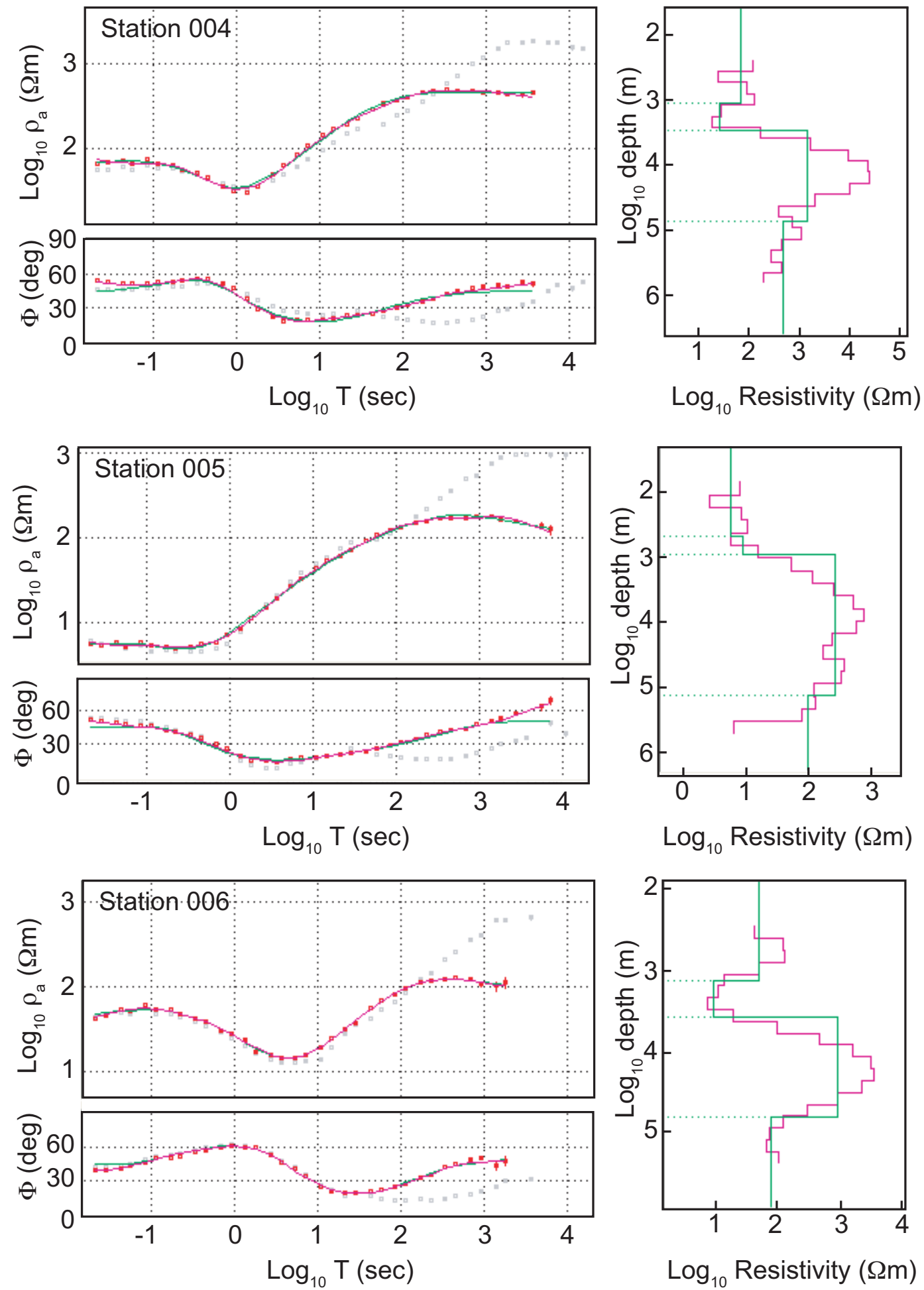

Figure 4.23: 1D models for stations 004-006. On the left TM mode apparent resistivity and phase data are shown as red dots and the TE data are shown in grey. The pink line is fit to the data for the 1D Occam model also in pink on the left. The green line is for a 4-layer resistivity model. 
tivity begins to increase deepens to the south from $\sim 600 \mathrm{~m}$ at station 001 to $\sim 2800$ $\mathrm{m}$ at station 006. This is in close agreement with sediment thickness based on seismic data and drill holes in the region (figure 4.5 and appendix A table A.3). Resistivity increases rapidly to a maximum of the order of $1000 \Omega \mathrm{m}$. This is typical of New Zealand's greywacke basement rocks [Ingham et al. 2001]. The model at station 003 has an anomalous structure with a local low in the resistivity of $\sim 100 \Omega \mathrm{m}$ at $10 \mathrm{~km}$ depth. The 1D inversion at this station has a poorer data fit than the other stations. This station is closest to the expected location of the TRL and therefore data at this station may be influenced more strongly by $2 \mathrm{D}$ structure.

Figure 4.24 shows a contour plot of the shallow Occam's inversion models along a N$\mathrm{S}$ profile and shows the thickening of the low resistivity region interpreted to be the Wanganui Basin sediments.

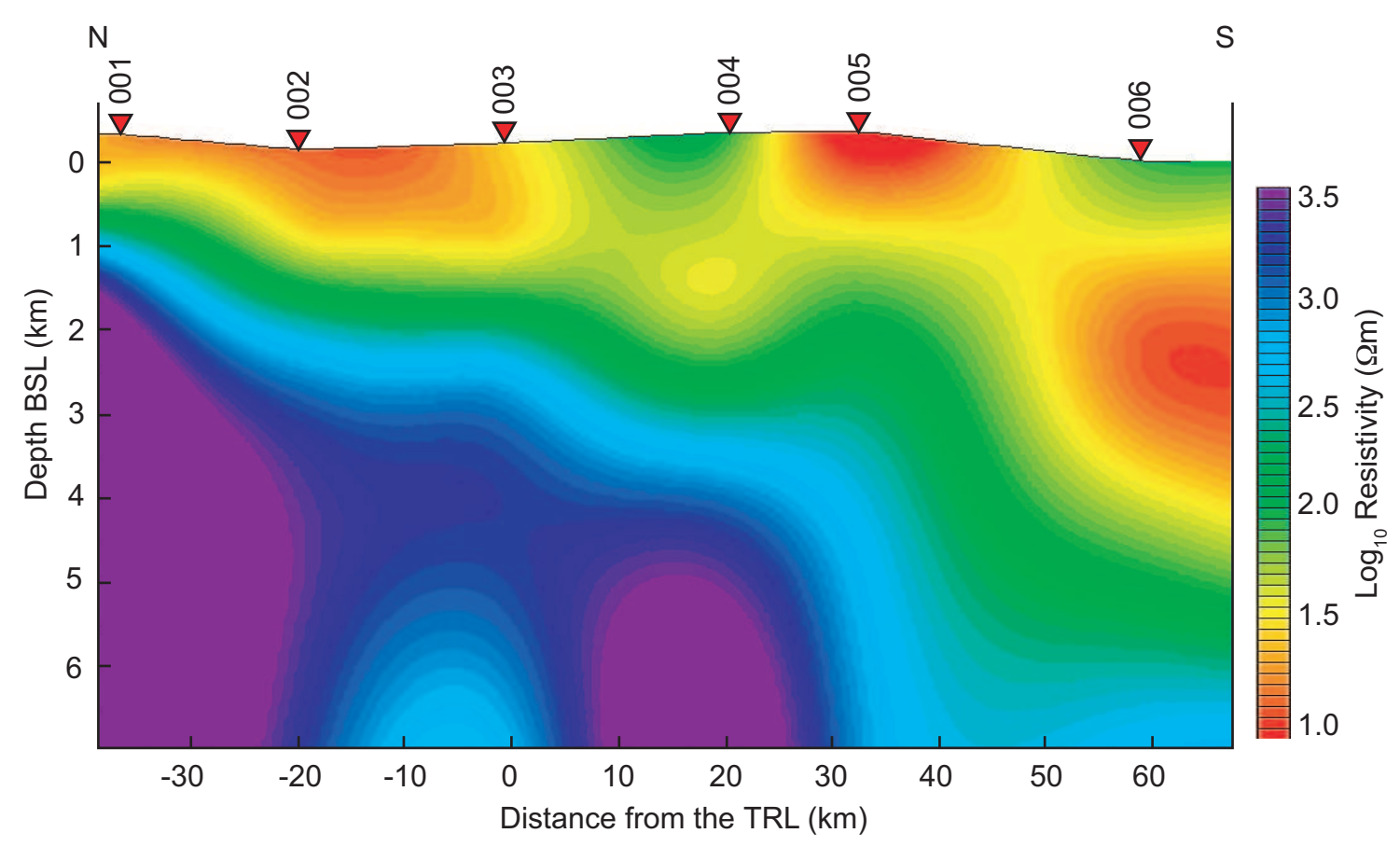

Figure 4.24: Pseudo section of shallow 1D Occam models. This section is formed by stitching together the TM mode 1D models from each station along the N-S profile. Red and yellow colours indicate a low resistivity region. Blue and purple indicate high resistivity.

The WinGLink software also has a layered model inversion that allows sharp resistivity contrasts. For this type of inversion the starting model becomes important and therefore the results of the Occam inversion are used as a guide for a starting model. The starting model has been further restricted to 4 layers with 2 near surface layers $(<5000$ $m$ depth) representing the high and low resistivity sediments and two deep layers to represent the basement and mantle. No restrictions are placed on layer thickness or resistivity for the inverted model. A minimum data error floor of $5 \%$ is also introduced to account for the uncertainty in data errors. This equates to \pm 0.022 in the $\log _{10}$ of the 
apparent resistivity and $\pm 1.43^{\circ}$ in phase. Figures 4.22 and 4.23 also show the results of a layered inversion after 10 iterations. The data show a reasonable fit using a 4-layer model. With the exception of station 005 the top layer has a higher resistivity than the second layer. The combined thickness of these two layers, interpreted to be the basin sediments, increases from $\sim 800 \mathrm{~m}$ at station 001 in the north to $\sim 3600 \mathrm{~m}$ at station 006 in the south. The layered model has a poorer fit to the data than the smooth Occam model, however the main features of the apparent resistivity and phase of the TM mode can be duplicated with a 4-layer model.

\subsubsection{D modelling}

As discussed previously (section 4.3.3), longer period data indicate there is an approximately 2D structure at depth. Data have therefore been inverted using a 2D extension of the 1D Occam's inversion [Constable et al. 2007, deGroot-Hedlin and Constable 1990]. This method parameterizes the $2 \mathrm{D}$ model using two model meshes. The first is a regularisation mesh, which consists of a grid of rectangular prisms with uniform resistivity. This grid is terminated at its boundaries by laterally elongated prisms at either side (layered boundary) and prisms elongated with depth at the bottom (half space boundary). The second mesh is a finite element mesh used to calculate the forward model. The forward model used here is the finite element model of Wannamaker et al. [1987], which solves for a primary and secondary electromagnetic field. The primary field describes the response to a 1D model and the secondary field describes the 2D departure from it.The finite element mesh is comprised of rectangular elements of a constant width in any given column and a constant height in any given row. The grid of the finite element mesh must include the grid of the regularisation mesh. Due to the logarithmic spacing and diffusive nature of the data, a logarithmic depth scaling is used for both meshes and prism width of the regularisation mesh is also allowed to increase with depth.

As with the 1D Occam's inversion the 2D code (Occam2D) seeks a smooth minimumstructure model. The smoothness of the model is calculated in a similar fashion to that used for the 1D case using a roughness parameter. The first derivative roughness parameter must now be calculated in two dimensions:

$$
R=\left\|\partial_{y} m\right\|^{2}+\left\|\partial_{z} m\right\|^{2}
$$

where $m$ is the vector of model parameters, $\partial_{y}$ is the roughening matrix, which differences the model parameters of laterally adjacent prisms and $\partial_{z}$ is a roughening matrix, which differences the model parameters of vertically adjacent prisms. A weighted root 
mean square misfit is used to determine the goodness of fit:

$$
r m s=\sqrt{\frac{\|W d-W F[m]\|^{2}}{N}}
$$

where $d$ is a vector of observed data values, $F[m]$ is a vector of predicted values calculated using the forward model, $N$ is the number of observations and $W$ is a diagonal weighting matrix:

$$
W=\operatorname{diag}\left\{1 / \sigma_{1}, 1 / \sigma_{2}, \ldots 1 / \sigma_{N}\right\}
$$

where $\sigma_{j}$ is the uncertainty in the $j$ th datum.

Initial iterations seek to reduce the misfit to a target value. Once this misfit level has been reached the inversion seeks a minimum roughness for that misfit. There is a trade-off between the goodness of fit and smoothness of the model. In order to allow the inversion program to remove unnecessary structure and produce a smooth model the target misfit should be set at least $25 \%$ higher than the minimum possible misfit [Constable 1994].

\section{Inversion parameters}

Figure 4.25 shows the 2D starting model used for this study. The model is oriented along a profile with an azimuth of $10^{\circ}$ and consists of a 60 layer regularisation mesh and an $81 \times 66$ finite element mesh. At the surface the regularisation prisms have widths of $9.8-6.5 \mathrm{~km}$ (half the station spacing to a maximum of $10 \mathrm{~km}$ ) and a thickness of $100 \mathrm{~m}$ ( $<$ the skin depth at the shortest period). Since the short period data are approximately $1 \mathrm{D}$, lateral variation should be minimal at shallow depths. Along with reduced lateral resolution of MT data with depth this justifies the use of the widely spaced regularisation mesh. The model extends laterally $60 \mathrm{~km}$ beyond the stations to ensure that the boundary conditions do not affect the inversion to at least $60 \mathrm{~km}$ depth. The model space extends to $1245 \mathrm{~km}$, well beyond the maximum skin depth of the data (appendix D table D.1). The finite element mesh is finer than the regularisation mesh; the minimum vertical spacing of the elements is $50 \mathrm{~m}$ or approximately $1 / 3$ of the skin depth of the highest frequency data.

The Occam2D code has been used to invert for the phase and resistivity of both the TE and TM modes simultaneously. Data have been excluded where the phase or apparent resistivity undergoes a sharp change or where there is a sudden increase in data uncertainty. In general data with a period range of $0.02-3640 \mathrm{~s}$ have been used in the inversion. Four sets of inversions were run:

1. Inversion using data from all 6 stations with no static shift. 


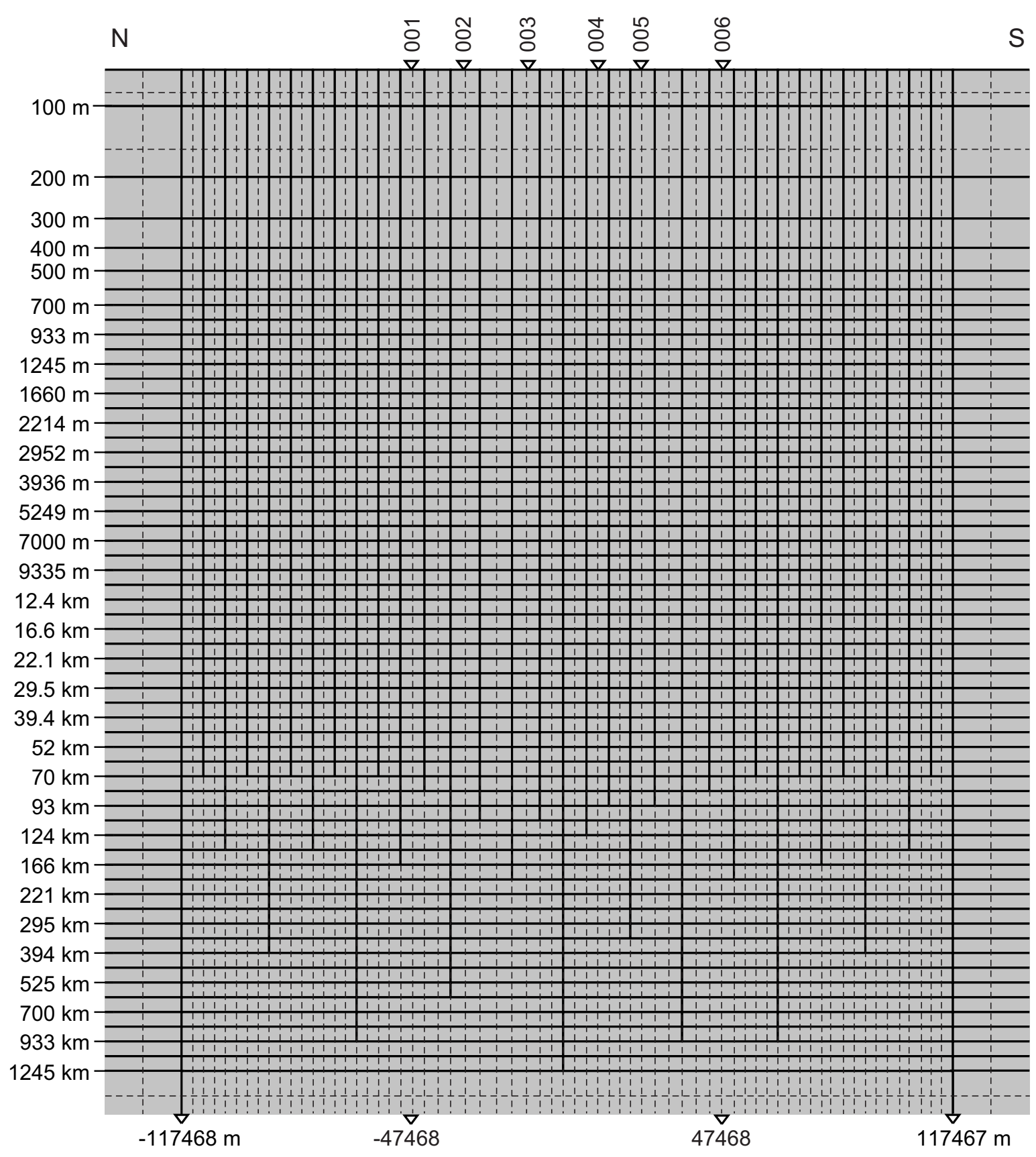

Figure 4.25: Input mesh for MT 2D-inversion. Solid lines represent the regularisation mesh. Each block of this mesh has a constant resistivity initially set to $100 \Omega \mathrm{m}$. The finite element mesh is overlaid as dashed lines. Side and bottom boundary blocks extend more than $1000 \mathrm{~km}$ beyond the limits of this figure. 
2. Inversion using data from all stations except 005 with no static shift.

3. Inversion using data from all stations and inverting for static shift.

4. Inversion using data from all stations except 005 and inverting for static shift.

To determine a reasonable target misfit for each inversion a preliminary inversion with a small target misfit was allowed to run until a minimum achievable misfit was obtained. The inversion was then run again using a target misfit $\sim 25 \%$ higher than the minimum misfit.

As previously noted the calculated data errors are likely to be underestimated. The first set of inversions were run once using the calculated errors and once with a $5 \%$ error floor. For the calculated errors the minimum achievable rms misfit is 3.8. Using a minimum error of $5 \%$ reduces the minimum achievable misfit to 1.6. Target rms misfits were set to 4.8 and 2.0 for inversions using calculated and $5 \%$ errors respectively. Both inversions were allowed to run for 90 iterations. Roughness values of the final models were then compared. Using a minimum $5 \%$ error produced the smoothest model with a roughness value of 56 . Using the calculated errors the roughness was 89. For inversion sets $2-4$ a $5 \%$ minimum error has been used.

As previously noted the apparent resistivity and phase at station 005 are anomalous. Data from this station have therefore been removed from inversion sets 2 and 4 . Inversion sets 3 and 4 invert for static shift as well as resistivity. Static shifts are expected to be small since short period resistivities are within the range expected for Wanganui Basin sediments. Allowing static shift to be a free parameter for both modes at all stations results in large downward static shifts for all stations. In order to restrict static shift parameters to small values the static shifts for the TE mode were initially set as a free parameter and no static shift is allowed for the TM mode. The static shift results from this initial step are used as set static shifts for both the TE and TM mode. Static shifts for the TE mode are inverted for first since this mode is less sensitive to lateral changes in resistivity structure and therefore lateral changes in apparent resistivity are less likely to be the result real structure.

\section{Inversion results}

The best-fit model to all six stations was achieved by including static shifts in the inversion. Figures 4.26 and 4.27 show the best-fit model. The remaining models and their data fits can be found in appendix D.

Using the calculated errors produced the worst fit especially at stations 001, 003 and 004 (figures D.2 and D.3). Using a 5\% minimum error improves the data fit at all stations. The increase in data error allows the inversion to fit phase and resistivity data more easily. Forward models of $\rho_{y x}$ (TE mode data) still do not fit the data well 
at station 001-003 (figure D.5). Allowing for static-shift greatly improves the data fit for station 001-003 (figure 4.28). Table 4.1 gives the static-shift inversion results for each station. Static-shifts less than 0.022 are less than the $5 \%$ minimum data error

Table 4.1: MT station static shifts

\begin{tabular}{|c|c|}
\hline Station & $\log _{10}(\Omega \mathrm{m})$ \\
\hline 001 & -0.076 \\
002 & -0.179 \\
003 & 0.181 \\
004 & -0.108 \\
005 & 0.001 \\
006 & 0.017 \\
\hline
\end{tabular}

in resistivity. Including static-shift in the inversion also allows the target misfit to be reduced to 1.5 without compromising model smoothness. Excluding station 005 from the inversions does not improve the model fit but it does reduce the roughness of the model.

Three features are consistent for all inversions models. The first is a near surface layer of low resistivity $(<20 \Omega \mathrm{m}$ ) (figure 4.26 ), which was also seen in the $1 \mathrm{D}$ inversion. The base of this low resistivity layer deepens to the south from $700 \mathrm{~m}$ at station 001

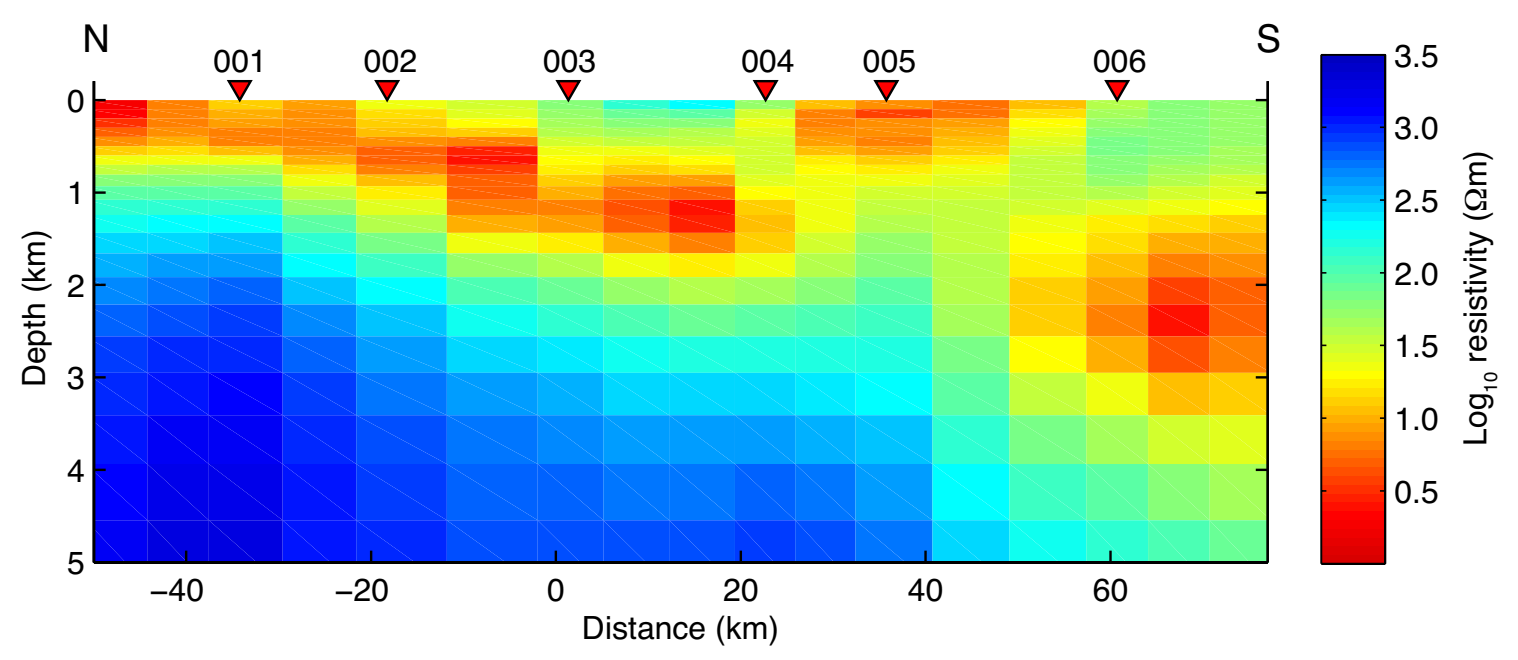

Figure 4.26: Shallow 2D resistivity structure for inversion using a 5\% minimum data error and including static-shifts. Horizontal distances are measured from the TaranakiRuapehu line.

to $\sim 3 \mathrm{~km}$ at station 006 . The only break in this trend is at station 005 where the low resistivity layer shallows. The second feature is a high resistivity layer $(>1000 \Omega \mathrm{m})$ at crustal depths, which deepens to the south. And lastly there is a low resistivity $(\sim 100$ $\Omega \mathrm{m})$ body at mantle depths. This body is generally shallower at the northern end of the profile (figure 4.27). 


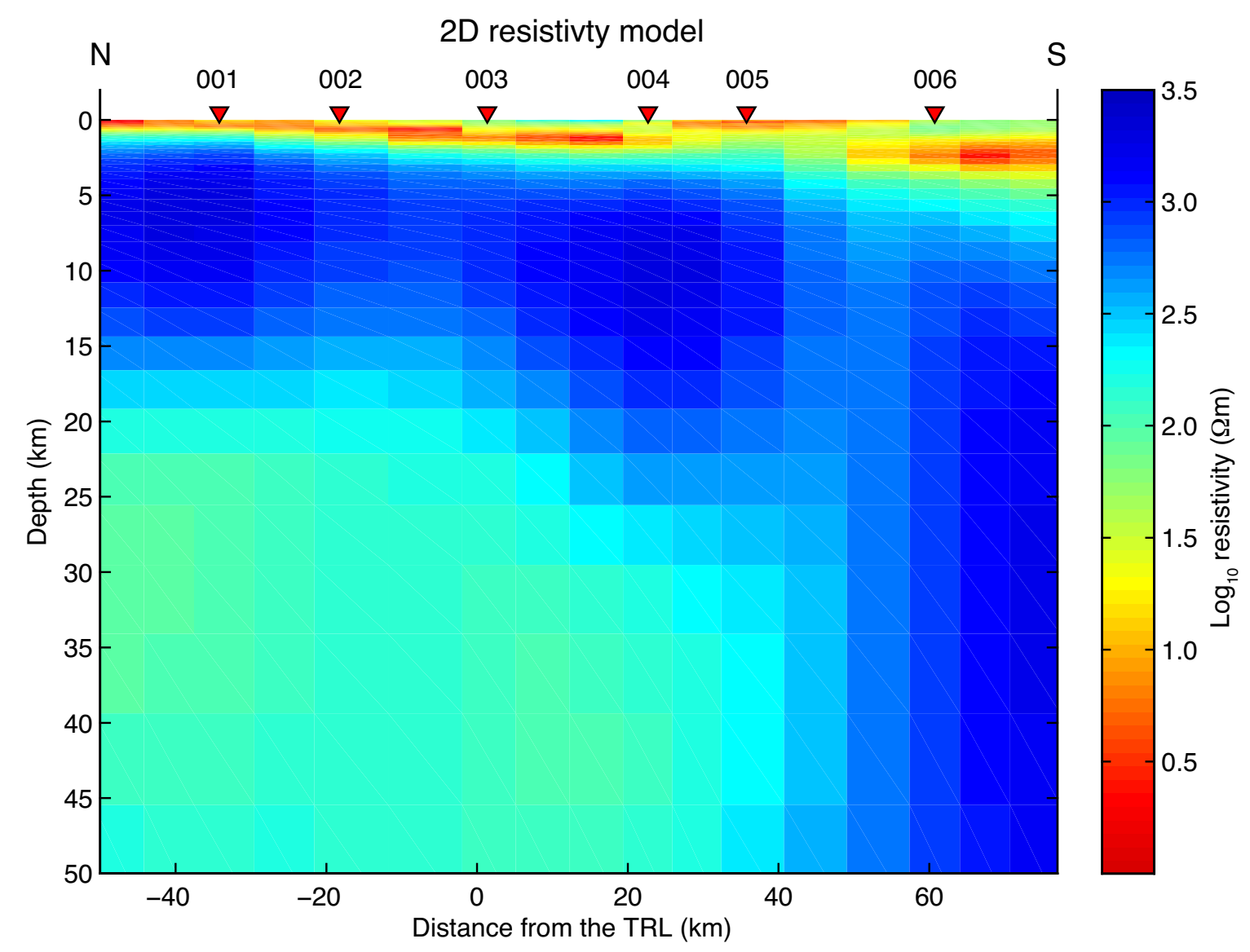

Figure 4.27: Best fit 2D resistivity model from inversion including static-shifts and using a $5 \%$ minimum error in the data. The forward model for this section has an rms misfit of 1.5 and the model roughness parameter is 54 . 
The best-fit model (figure 4.27) shows the low resistivity body in the mantle below 20 $\mathrm{km}$ depth north of station 003. South of this station this zone of low resistivity deepens and is completely absent below station 006. Figures 4.28-4.29 show the model fit to the data. At station 001 the phase data are poorly fitted for periods between $0.3 \mathrm{~s}$ and $3 \mathrm{~s}$. This period range is within the dead band and phase data vary less smoothly than data either side. Over this range $\phi_{x y}$ drops to very small phase angles. The phase data are proportional to the derivative of the apparent resistivity (4.2.20) and a very low phase indicates a sharp decrease in resistivity. The smooth models produced by the Occam inversion do not model sharp resistivity boundaries well.

Lateral sensitivity of the model was tested by shifting the mantle body north and south and running a forward model. A lateral shift of up to $7 \mathrm{~km}$ does not significantly change the fit to the data, but a shift of more than this results in $>10 \%$ increase in misfit of the TM mode. Vertical sensitivity was tested by shifting the mantle body up and down. Shifting the mantle body vertically more than $10 \mathrm{~km}$ results in $>10 \%$ increase in the misfit of the TE mode.

\subsection{Interpretation}

The low resistivity layer at the surface is interpreted as Miocene-Pleistocene sediments; these deepen southward toward the Wanganui basin, which contains over $4 \mathrm{~km}$ of shallow marine sediments [Anderton 1981]. Figure 4.30 shows the estimated basement depth along the MT profile based on Anderton [1981] and a compilation of oil industry data [e.g. Gerrard 1971, St John 1964, Watson and Allen 1964]. There is a good correlation between estimated basement depth and a change to increasing resistivity with depth.

The high resistivity layer beneath the sediments is interpreted as crust. A typical crustal resistivity is of the order of 1000 Sm [Schilling et al. 1997]. DC resistivity surveys indicate that below $5 \mathrm{~km}$ the greywacke basement in New Zealand has typical resistivities of $>1000 \Omega \mathrm{m}$ [Risk et al. 1993]. At the southern end of the resistivity profile this layer extends to depths $>70 \mathrm{~km}$ depth. Crustal thickness below the depocenter of the Wanganui basin is estimated to be $\sim 40 \mathrm{~km}$ [Holt and Stern 1994]. This means that the mantle below the southern end of the MT line also has resistivities $>1000 \Omega \mathrm{m}$. From laboratory measurements, common mantle minerals have resistivities $>1000 \Omega \mathrm{m}$ at temperatures less than $1100^{\circ} \mathrm{C}$ [Constable 2006, Ledo and Jones 2005] making the crust/mantle boundary difficult to identify.

At the northern end of the profile the low resistivity body is interpreted to be within the mantle. Receiver function analysis indicates the Moho is at $25 \mathrm{~km}$ depth in this region (see chapter 2). The region of low resistivity is north of the TRL and is coincident with a region of high seismic attenuation associated with elevated temperatures (chapter 3 ). 

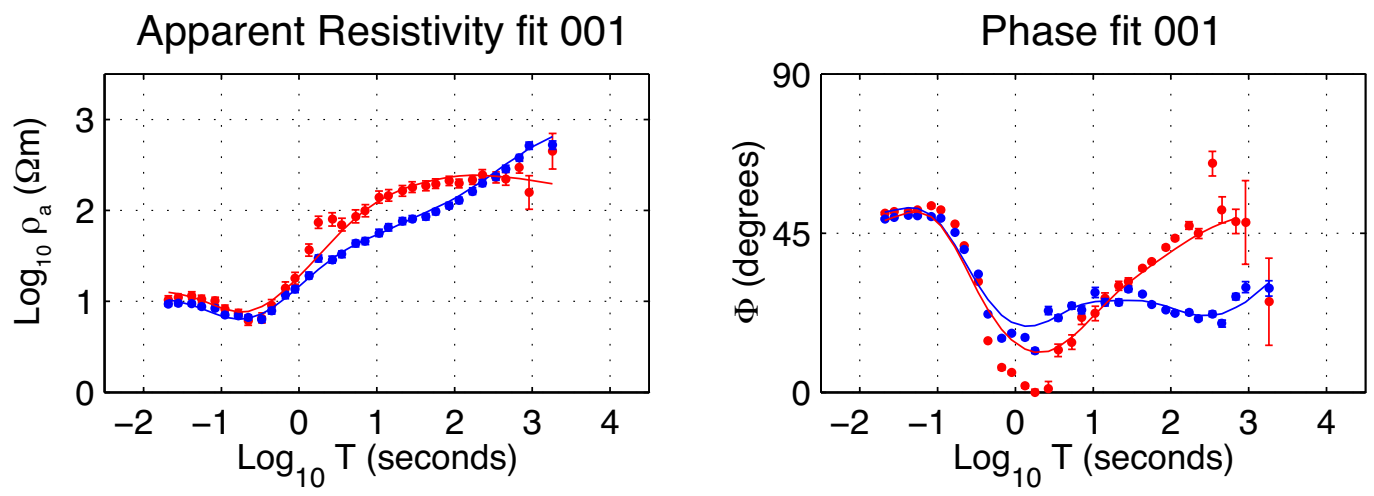

Apparent Resistivity fit 002
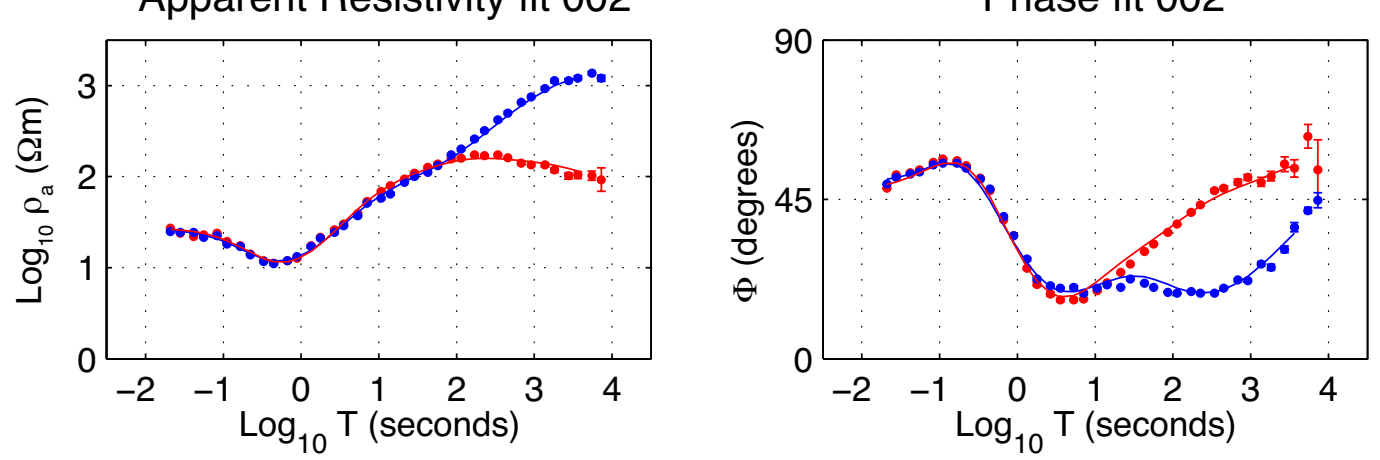

Apparent Resistivity fit 003
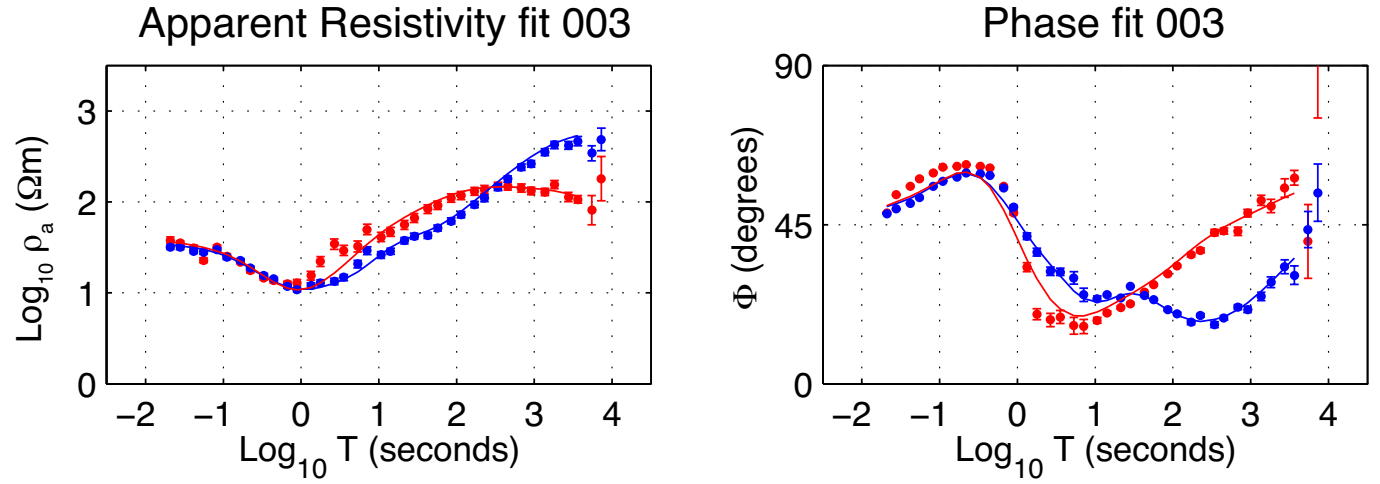

Figure 4.28: Forward model fit to the data at stations 001-003 for the best-fit model shown in figure 4.27 (5\% minimum error and static-shift). The forward model for the TM mode $\left(\rho_{x y}\right)$ is shown as a red line and the forward model for the TE mode $\left(\rho_{y x}\right)$ is shown as a blue line. 

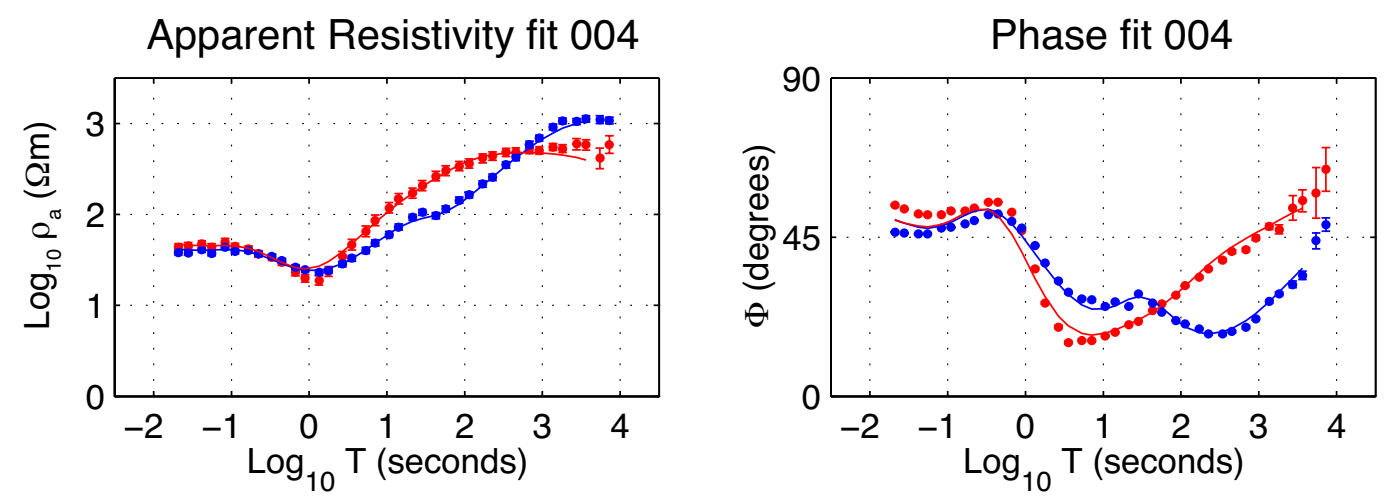

Apparent Resistivity fit 005
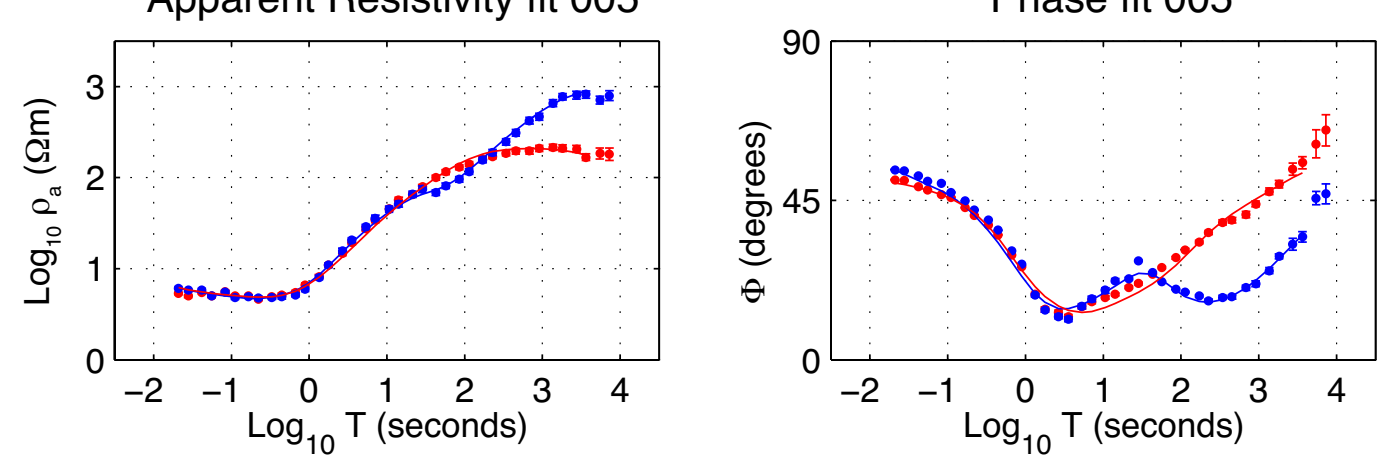

Apparent Resistivity fit 006
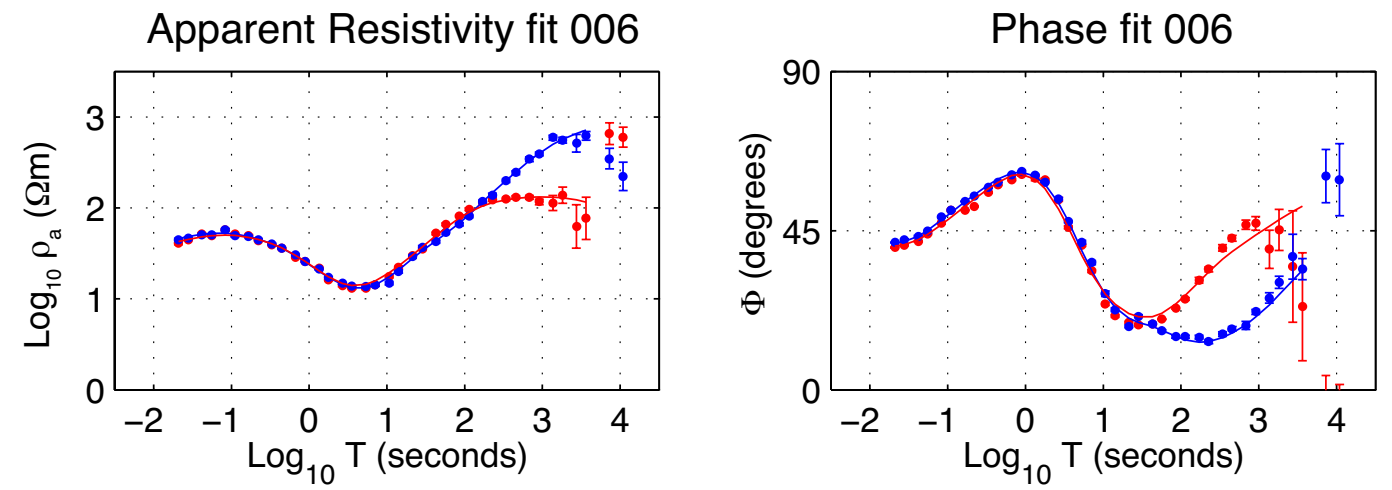

Figure 4.29: Forward model fit to the data at stations 004-006 for the best-fit model shown in figure 4.27 (5\% minimum error and static-shift). The forward model for the TM mode $\left(\rho_{x y}\right)$ is shown as a red line and the forward model for the TE mode $\left(\rho_{y x}\right)$ is shown as a blue line. 


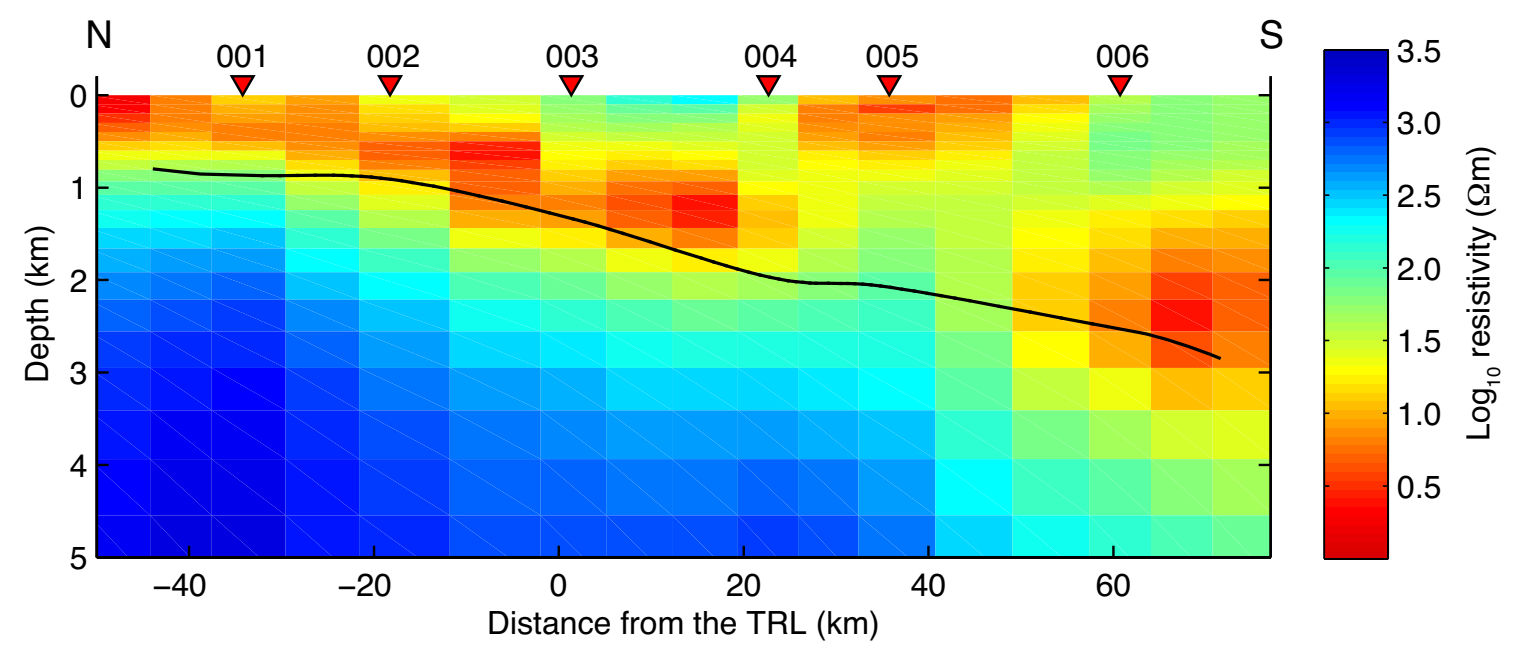

Figure 4.30: Estimated basement depth along the MT profile. The black line indicates the depth to basement. Basement depth is closely matched by the change from decreasing to increasing resistivity with depth.

The electrical resistivity of dry peridotite decreases with increasing temperature. For a dry mantle, resistivities of $100 \Omega \mathrm{m}$ equate to temperatures in excess of $1300^{\circ} \mathrm{C}$. Recent advances in laboratory studies of mantle minerals show that the presence of water in nominally anhydrous mantle minerals can significantly decrease the resistivity of the mantle. Wang et al. [2006] propose a relationship between conductivity, temperature and water content for olivine.

$$
\sigma_{H}=A C_{w}^{r} \exp \left(-\frac{H^{*}}{R T}\right)
$$

Where $A$ and $r$ are constants (1000 $\mathrm{Sm}^{-1}$ and 0.62 respectively), $H^{*}$ is the activation enthalpy $\left(87 \mathrm{~kJ} \mathrm{~mol}^{-1}\right), R$ is the gas constant, $T$ is the temperature $(\mathrm{K})$ and $C_{w}$ is the water content $(w t \%)$. At $30 \mathrm{~km}$ depth the water storage capacity of peridotite is in the range 0.01-0.03 wt\% [Bell and Rossman 1992, Hauri et al. 2006, Hirth and Kohlstedt 2003]. Using this constraint, a resistivity of $100 \Omega \mathrm{m}$ equates to a temperature range of $850-940^{\circ} \mathrm{C}$.

At the southern end of the MT profile the subducting Pacific Plate is shallower than at the northern end [Ansell and Bannister 1996]. It therefore seems likely that this region has also undergone some amount of hydration. Using the same water content range as the northern end of the profile and a resistivity of $1000 \Omega \mathrm{m}$ returns a temperature range of $630-680^{\circ} \mathrm{C}$. 


\section{Chapter 5}

\section{DISCUSSION}

Three independent data sets have been used to examine the back-arc region of North Island New Zealand. The data sets reveal coincident changes in crustal thickness, seismic attenuation and mantle resistivity at the Taranaki-Ruapehu Line (TRL). North of the TRL crust is thin $(\sim 25 \mathrm{~km})$, seismic attenuation is high $\left(1000 / Q_{p} \approx 1.9\right)$ and the electrical resistivity of the mantle is low $(\sim 100 \Omega \mathrm{m})$. South of the TRL the crust has a minimum thickness of $32 \mathrm{~km}$, seismic attenuation is low $\left(1000 / Q_{p}<1\right)$ and electrical resistivity is high $(>1000 \Omega \mathrm{m})$. The following is a discussion of the implications of these observations for mantle composition and tectonics.

\subsection{Temperature, melt and water content}

Both seismic attenuation and electrical resistivity indicate the presence of hot hydrated mantle below the Central Volcanic Region (CVR) and the northwestern North Island (NWNI). Below the CVR mantle temperatures just above melting are inferred from seismic attenuation $(T / T m=1.02)$. Melts in the uppermost mantle are buoyant [Ochs and Lange 1999] and therefore melting in the mantle should also be evident at the surface. This is certainly the case for the CVR, which is the most productive silicic volcanic system on earth [Wilson et al. 1995]. Below the NWNI temperatures inferred from both electrical resistivity and seismic attenuation are just below melting temperature $(T / T m=0.87-0.97)$. For a water saturated mantle this equates to 840 $940^{\circ} \mathrm{C}$. This is in good agreement with temperature estimates at the Moho based on heat flow [Pandey 1981a], supporting the assumption of a water saturated mantle.

Mantle temperatures of $\sim 650^{\circ} \mathrm{C}$ below the South Wanganui Basin are inferred from the mantle resistivity. These results are consistent with temperature estimates from heat flow data in the region [Pandey 1981a]. South Wanganui Basin mantle temperatures are close to steady state temperatures expected at the base of continental crust [McKenzie et al. 2005], but suggest there is a $\sim 290^{\circ} \mathrm{C}$ temperature difference across the TaranakiRuapehu Line. 
Temperature estimates for the mantle north of the TRL fit well with observations of other back-arcs [Currie and Hyndman 2006]. Temperature estimates for the mantle south of the TRL however, are much lower than usually observed in back-arc regions.

Isostatic modelling of the North Island rock uplift indicates the presence of a buoyant low-density body below the CVR and the NWNI [Pulford and Stern 2004]. The estimated reduction in mantle density required is $66 \mathrm{kgm}^{-3}$ below the CVR and 35 $\mathrm{kgm}^{-3}$ below the NWNI. The change in density, $\Delta \rho$, caused by thermal expansion can be calculated using equation 5.1.1 [Turcotte and Schubert 1982]

$$
\Delta \rho=\rho \alpha \Delta T
$$

where $\rho$ is the standard mantle density $\left(3300 \mathrm{kgm}^{-3}\right), \Delta T$ is the change in temperature and $\alpha \approx 3.5 \times 10^{-5} \mathrm{~K}^{-1}$ is the expansion coefficient for the mantle [Bouhifd et al. 1996]. The temperature difference between the South Wanganui Basin and the NWNI $\left(\Delta T=290^{\circ} \mathrm{C}\right)$ returns $\Delta \rho=33 \mathrm{kgm}^{-3}$. The buoyancy force below this region can be accounted for by temperature alone. The temperature difference between the CVR and the South Wanganui basin is $\sim 340^{\circ} \mathrm{C}$. This returns a density difference of $\sim 40$ $\mathrm{kgm}^{-3}$. Thermal expansion alone can not account for the buoyancy of the CVR. Mantle melts have a lower density than solid upper mantle materials and therefore a $2 \%$ melt will reduce mantle density by about a further $10 \mathrm{kgm}^{-3}$. In addition the residuum left behind after melt extraction is less dense that the original rock [Schutt and Lesher 2006] and the presence of water will also reduce density [Ochs and Lange 1999]. The remaining density deficit may therefore be accounted for by the presence of water and eruptive melt removal.

\subsection{The Taranaki-Ruapehu Line}

Thinned crust and high seismic attenuation observed north of the TRL are common features of many back-arc basins [Wiens et al. 2006]. The abrupt change in these properties observed at the TRL is more unusual and more difficult to explain. Changes in seismic attenuation, electrical resistivity and crustal thickness across the TaranakiRuapehu line occur over a short horizontal distance $(<30 \mathrm{~km})$. Sharp changes in crustal thickness are usually associated with active tectonic processes such as crustal scale strike-slip faulting [e.g. Zhu 2000]. There is no evidence for such a fault along the Taranaki-Ruapehu line. Crustal thinning is typically associated with extension, yet crustal thinning of the NWNI also seems to have occurred without significant extension [Stern et al. 2006]. The lack of any surface expression of this change in crustal thickness suggests that it was formed from below by mantle processes. 
Pollack and Chapman [1977] argue that the thickness of the strong, high viscosity lithosphere can be determined by the depth to $T / T m=0.85$. By this definition the lithospheric mantle north of the TRL is entirely missing (figure 5.1). Conversely, south of the TRL there is no asthenosphere in the mantle wedge.

Recent relocations of earthquakes in the vicinity of the this study are shown in figure 5.1 [Reyners et al. 2007]. A region of deeper seismicity extending to depths of $\sim 50$ $\mathrm{km}$ is clustered around the TRL. Results of this study indicate that earthquakes below $\sim 25 \mathrm{~km}$ in this region are located within the mantle. Previous studies have attributed these earthquakes to a change in crustal thickness in this region [Reyners et al. 2006, Sherburn and White 2005] but have located these earthquakes in the lower crust. Stern et al. [2006] suggest that these earthquakes are the result of horizontal density contrasts across the steep crustal thickness boundary of the TRL. Based on the northern extent of these deep earthquakes Sherburn and White [2005] place the TRL boundary further to the north based on the premise that earthquakes do not occur in the mantle. This point is still under debate but there is evidence of earthquakes within the mantle in other parts of the world [Chen and Yang 2004].

The rapid uplift of the North Island over the last 5 Ma [Pulford and Stern 2004] suggests that hot buoyant mantle was emplaced below the CVR and the NWNI much more rapidly than can be explained by conductive thermal erosion, which would take of the order of $60 \mathrm{Ma}$ [Turcotte and Schubert 1982]. Currie and Hyndman [2006] propose vigourous convection as a mechanism for rapid thinning of lithosphere in back-arc regions. They argue that a broad, hot back-arc may be a fundamental characteristic of subduction zones. This however is not the case for the back-arc region south of the TRL. Both seismic attenuation and electrical resistivity suggest that the mantle below the South Wanganui Basin is cold. Crust below the basin appears to be thickened and down-warped [Stern and Davey 1990]. The basin correlates with a deep low in the isostatic gravity anomaly indicating the lithosphere is also thickening beneath the basin [Ewig and Stern 2006].

Stern et al. [2006] provide one possible explanation for the changes seen in the lithosphere across the TRL. They suggest a series of Rayleigh Taylor instabilities are responsible for the convective removal of the lithospheric mantle, and possibly the lower crust, below the NWNI. These instabilities form as a lithospheric drip which warps the crust downward and thins the lithosphere at its peripheries. Eventually the drip falls off, releasing the down-warped crust and causing rapid uplift. Lithospheric thickening of the NWNI during the Miocene provides the initial conditions required for Rayleigh Taylor instabilities to develop. A southward progression of these instabilities is used to explain the southward migration of sedimentary basins above the lithospheric downwelling and can explain the current thickening of the lithosphere south of the TRL. 


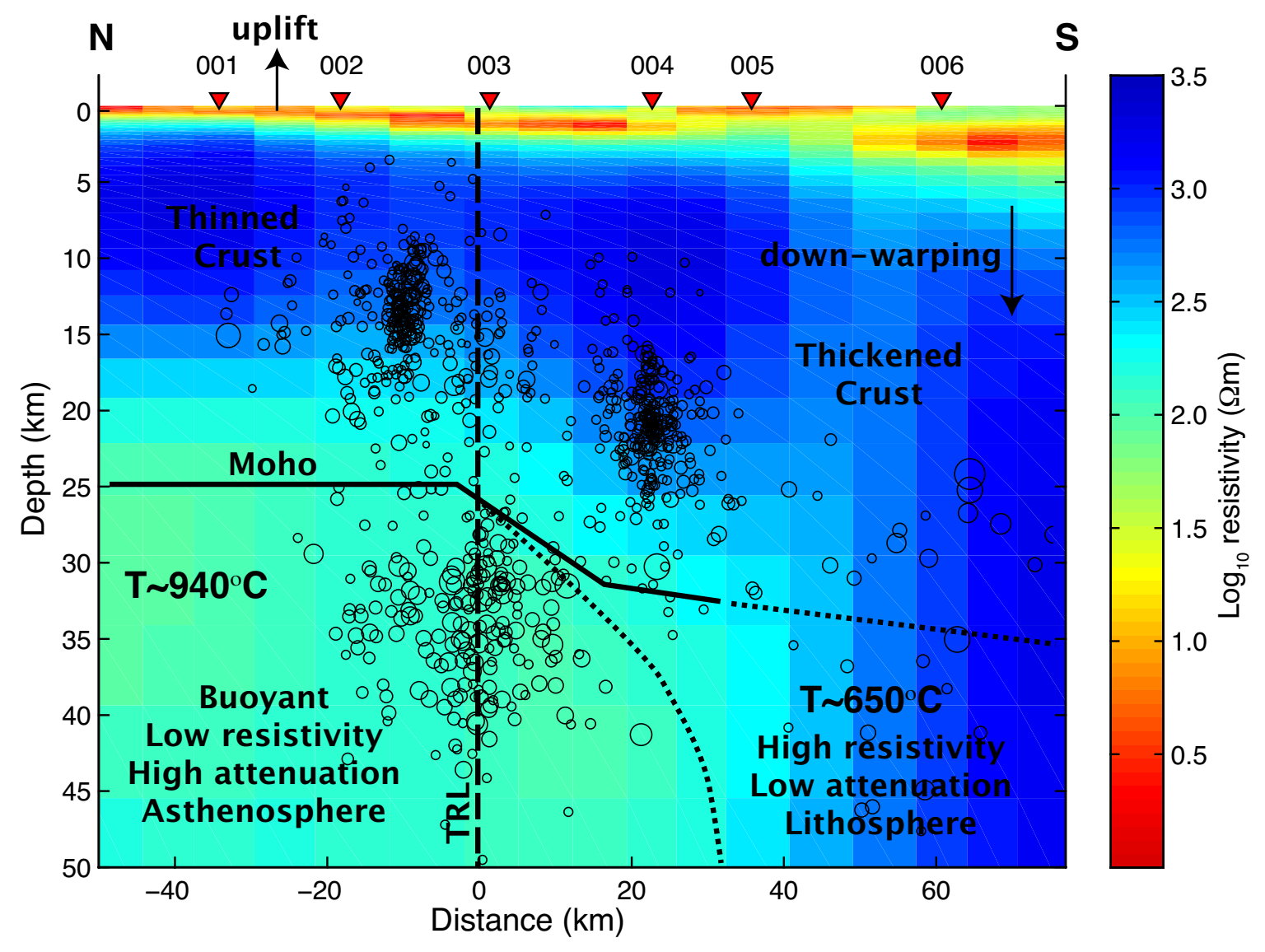

Figure 5.1: Taranaki-Ruapehu Line. Features of the crust and mantle either side of the TRL overlaid on the 2D resistivity model. The solid black line is the Moho inferred from receiver function analysis. Black circles indicate the depth of seismicity projected along the profile [Reyners et al. 2007]. Circle sizes are propotional to magnitude. The region north of the TRL has thinned crust and is underlain by hot low-density asthenosphere with low resistivity and high attenuation. The region south of the TRL has thickened crust and is underlain by cool high density lithospheric mantle. 
Similar mechanisms have been proposed for the removal of eclogitized crust and upper mantle below other continental regions that have undergone a period of compression followed by rapid uplift [Molnar and Garzione 2007, Molnar and Jones 2004]. The Sierra Nevada is one such region that has a number of similarities with the western North Island. The absence of lithospheric mantle below the high Sierra has been inferred from low Pn velocities, high attenuation and low resistivities [Boyd et al. 2004, Jones et al. 1994, Park et al. 1996]. A lithospheric down-welling beneath the adjacent Great Valley is inferred from high $\mathrm{P}$-wave velocities and negative gravity anomalies [Gilbert et al. 2007, Zandt 2003]. Also observed are deep crustal earthquakes above the foundering lithosphere similar to those seen below the Wanganui Basin in New Zealand.

Two alternative views on the origin of anomalous mantle below northwestern North Island have been put forward. Kamp and Furlong [2006] propose that delamination of the lithosphere has occured in order to accomodate the pacific plate as subduction has propagated southward. Observations for this theory would be similar to that of Stern et al. [2006]. Reyners et al. [2006; 2007] attribute the changes in seismic properties across the TRL to changes in mantle flow due to a change in crustal thickness. They suggest that north of the TRL fluids are entrained into the mantle by the subducting slab and a return flow introduces partial melt into the mantle wedge. South of the TRL they propose that the thickened crust chokes off this return flow. This theory does not however provide an explaination for the origin of the change in crustal thickness. 


\section{Chapter 6}

\section{CONCLUSIONS}

Crust and uppermost mantle properties below the back-arc region of western North Island, New Zealand has been investigated using three independent geophysical methods; receiver functions, seismic attenuation and magnetotellurics.

Receiver functions reveal $\sim 7 \mathrm{~km}$ change in crustal thickness across the TaranakiRuapehu line (TRL), from $\sim 25 \mathrm{~km}$ below the northwestern North Island to $\geqslant 32$ $\mathrm{km}$ below the southwestern North Island. P-wave attenuation is elevated north of the TRL $\left(1000 / Q_{p} \approx 1.9\right)$ and is compatible with a wet mantle at near solidus temperatures ( $\mathrm{T} \approx 0.97$ melting temperature). Attenuation decreases by at least a factor of 2 south of the TRL, to values closer to those expected for normal continental lithosphere $\left(1000 / Q_{p} \leqslant 1\right)$. A region of extremely high $\mathrm{P}$-wave attenuation $\left(1000 / Q_{p} \approx 5\right)$ is observed below the Central Volcanic Region. This value of attenuation is indicative of wet mantle at temperatures just above melting ( $\mathrm{T} \approx 1.02$ melting temperature). 2D modelling of magnetotelluric data reveals a region of low electrical resistivity (100 $\Omega \mathrm{m})$ in the mantle north of the TRL. Like the P-wave attenuation, this region of low resistivity can be explained by a water-saturated mantle at near solidus temperatures ( $\mathrm{T}=0.85-0.94$ melting temperature).

Observed changes in crustal thickness, attenuation and electrical resistivity occur at a common boundary (the Taranaki-Ruapehu Line). This boundary runs approximately east-west, perpendicular to the present day plate boundary and marks the southern limit of volcanism. The only surface expression of the TRL is the dome-like uplift of the North Island, which has previously been explained by the presence of a buoyant low-density mantle beneath the north-western North Island. Evidence of elevated temperatures and water content from this study are in agreement with this explanation.

The sudden transition displayed in all three data sets, but particularly the crustal thickness step seen in the receiver function, calls for a special explanation. Thermal processes are too diffuse to explain the step and instead a mechanical process is called for. One possibility is that the step was created by convective removal of thickened lithosphere. 


\section{Bibliography}

Abercrombie, R., Earthquake source scaling relationships from -1 to 5 ML using seismograms recorded at 2.5-km depth, Journal of Geophysical Research, 100 (B12), 24,015-24,036, 1995.

Abers, G., Array measurements of phases used in receiver-function calculations: Importance of scattering, Bulletin of the Seismological Society of America, 88(1), 313-318, 1998.

Anderton, P., Structure and evolution of the South Wanganui Basin, New Zealand, New Zealand Journal of Geology and Geophysics, 24(1), 39-63, 1981.

Ansell, J., and S. Bannister, Shallow morphology of the subducted Pacific plate along the Hikurangi margin, New Zealand, Phys. Earth Planet. Inter., 93, 3 - 20, 1996.

Audoine, E., M. K. Savage, and K. Gledhill, Seismic anisotropy from local earthquakes in the transition region from a subduction to a strike-slip plate boundary, New Zealand, Journal of Geophysical Research, 105(B4), 8013-8033, 2000.

Audoine, E., M. K. Savage, and K. Gledhill, Anisotropic structure under a back-arc spreading region, the Taupo Volcanic Zone, New Zealand, Journal of Geophysical Research, 109, doi:10.1029/2003JB002932, 2004.

Ave Lallemant, H., J.-C. Mercier, N. Carter, and J. Ross, Rheology of the upper mantle: inferences from peridotite xenoliths, Tectonophysics, 70, 85-113, 1980.

Bannister, S., M. Reyners, G. Stuart, and M. Savage, Imaging the Hikurangi subduction zone, New Zealand, using teleseismic receiver functions; crustal fluids above the forearc mantle wedge., Geophysical Journal International, 169(2), 602-616, doi: 10.1111/j.1365-246X.2007.03345.x, 2007.

Bell, D., and G. Rossman, Water in Earth's mantle: The role of nominally anhyrdous minerals, Science, 255, 1391-1397, 1992.

Bibby, H., T. Caldwell, F. Davey, and T. Webb, Geophysical evidence on the structure of the Taupo Volcanic Zone and its hydrothermal circulation, Journal of Volcanology and Geothermal Research, 68, 29-58, 1995. 
Bibby, H., T. Caldwell, and C. Brown, Determinable and non-determinable parameters of galvanic distortion in magnetotellurics, Geophysical Journal International, 163, 915-930, 2005.

Bock, G., and J. Clements, Attenuation of short-period P, PcP, ScP and pP waves in the earth's mantle, Journal of Geophysical Research, 87(B5), 3905-3918, 1982.

Boore, D. M., and W. Joyner, Site amplifications for generic rock sites, Bull. Seis. Soc. Amer., 87, 327-341, 1997.

Bouhifd, M., D. Andrault, G. Fiquet, and P. Richet, Thermal expansion of forsterite up to the melting point, Geophysical Research Letters, 23(10), 1143-1146, 1996.

Boyd, O., C. Jones, and A. Sheehan, Foundering lithosphere imaged beneath the southern Sierra Nevada, California, USA, Science, 305, 660-662, 2004.

Brune, J., Tectonic stress and the spectra of seismic shear waves from earthquake sources, Journal of Geophysical Research, 75, 4997-5007, 1970.

Brune, J., Tectonic stress and the spectra of seismic shear waves from earthquake sources; correction, Journal of Geophysical Research, 76, 5002, 1971.

Brune, J., R. Archuleta, and S. Hartzell, Far-field S-wave spectra, corner frequencies, and pulse shapes, J. Geophys. Res., 84(B5), 2262-2272, 1979.

Bryan, C., A. Hurst, S. Sherburn, H. Bibby, and S. Bannister, Shallow seismicity of the central Taupo Volcanic Zone, New Zealand: its distribution and nature, New Zealand Journal of Geology and Geophysics, 42, 533-542, 1999.

Cagniard, L., Basic theory of the magnetotelluric method of geophysical prospecting, Geophysics, 18, 605-635, 1953.

Cassidy, J. F., Numerical experiments in broadband receiver function analysis, Bulletin of the Seismological Society of America, 82(3), 1453-1474, 1992.

Chavez-Garcia, F., L. Sanchez, and D. Hatzfield, Topographic site effets and HVSR. A comparision between observations and theory, Bulletin of the Seismological Society of America, 86(5), 1599-1573, 1996.

Chen, W., and Z. Yang, Earthquakes beneath the Himalayas and Tibbet: Evidence for strong lithospheric mantle, Science, 304, 1949-1952, 2004.

Christensen, N., Poisson's ratio and crustal seismology, Journal of Geophysical Research, 101 (B2), 3139-3156, 1996.

Constable, C., Occam v2.xx release notes, Tech. rep., Institute of Geophysics and Planetary Physics, Scripps Institution of Oceanography, 1994. 
Constable, C., K. Key, and D. Myer, Occam v3.0, Website, http://marineemlab. ucsd.edu/Projects/Occam/, 2007.

Constable, S., SEO3: A new model of olivine electrical conductivity, Geophys. J. Int., 166, 435-437, 2006.

Constable, S., R. Parker, and C. Constable, Occam's inversion: a practical algorithm for generating smooth models from electromagnetic sounding data, Geophysics, 52(3), 289-300, 1987.

Coombs, D., C. Landis, J. Sinton, D. Borns, and D. Craw, The Dun Mountain ophiolite belt, New Zealand, its tectonic setting, constitution, and origin, with special reference to the southern portion, American Journal of Science, 276, 561-603, 1976.

Currie, C. A., and R. D. Hyndman, The thermal structure of subduction zone back arcs, Journal of Geophysical Research, 111, doi:10.1029/2005JB004024, 2006.

deGroot-Hedlin, C., and S. Constable, Occam's inversion to generate smooth, twodimensional models from magnetotelluric data, Geophysics, 55(12), 1613-1624, 1990.

Dueker, K., and A. Sheehan, Mantle discontinuity structure from midpoint stacks of converted P to S waves across the Yellowstone hotspot track, Journal of Geophysical Research, 102(B4), 8318-8327, 1997.

Dueker, K., and A. Sheehan, Mantle discontiuity structure beneath the Colorado Rocky Mountains and High Plains, Journal of Geophysical Research, 103(B4), 7153-7169, 1998.

Durek, J., and G. Ekstrom, A radial model of anelasticity consistent with long-period surface-wave attenuation, Bulletin of the Seismological Society of America, 86(1A), 144-158, 1996.

Dziewonski, A. M., and D. L. Anderson, Preliminary reference Earth model, Physics of the Earth and Planetary Interiors, 25, 297-356, 1981.

Eberhart-Phillips, D., M. Reyners, M. Chadwick, and J. Chiu, Crustal heterogeneity and subduction processes: 3-D Vp, Vp/Vs and Q in the southern North Island, New Zealand, Geophysical Journal International, 162, 270-288, doi:10.1111/j.1365-246X. 2005.02530.x, 2005.

Edbrooke, S. c., Geology of the Waikato area, Institute of Geological and Nuclear Sciences 1:250000 geological map 4, 2005.

Ewig, E., and T. Stern, Lithospheric shortening and ductile deformation in a back-arc setting: Wanganui Basin, New Zealand, EOS Transaction, American Geophysical Union, 87(52), Fall Meet. Suppl., Abstract T53B-1607, 2006. 
Faul, U., and I. Jackson, The seismological signature of temperature and grain size variations in the upper mantle, Earth and Planetary Science Letters, 234, 119-134, 2005 .

Faul, U., J. Fitz Gerald, and I. Jackson, Shear wave attenuation and dispersion in melt-bearing olivine polycrystals: 2. Microstructural interpretation and seismological implications, Journal of Geophysical Research, 109, doi:10.1029/2003JB002407, 2004 .

Flanagan, M., and D. Wiens, Attenuation of Broadband P and S Waves in Tonga: Observations of Frequency Dependent Q, Pure and Applied Geophysics, 153, 345375, 1998.

Fowler, C., The Solid Earth: an introduction to global geophysics, Cambridge University Press, Cambridge, U.K., 1990.

Frederiksen, A., and M. Bostock, Modelling teleseismic waves in dipping anisotropic structures, Geophysical Journal International, 141, 401-412, 2000.

Gamble, T., W. Goubau, and J. Clarke, Error analysis for remote reference magnetotellurics, Geophysics, 44(5), 959-968, 1979a.

Gamble, T., W. Goubau, and J. Clarke, Magnetotellurics with a remote magnetic reference, Geophysics, 44(1), 53-68, 1979b.

Garrick, R., and S. Gibowicz, Continuous swarm-like seismicity: the Wanganui, New Zealand, earthquakes, Geophys. J. R. astr. Soc., 75, 493-512, 1983.

GEOSYSTEM SRL, WinGLink user's guide, release 2.07.05b ed., 2005.

Gerrard, M., Tupapakurua-1, Petroleum Report Series PR266, ANZPAC Petroleum Corp, 1971.

Gilbert, H., C. Jones, T. Owen, and G. Zandt, Imaging Sierra Nevada lithsperic sinking, EOS, Transaction, American Geophysical Union, 88(21), 225-236, 2007.

Gledhill, K., R. Randall, and M. Chadwick, The EARSS digital seismograph: system description and field trials, Bulletin of the Seismological Society of America, 81, 1380-1390, 1991.

GNS Science, Eyrewell geomagnetism archive, Website, http://www.gns.cri.nz/ research/geomagnetism/archive/eyrewell, 2002.

GNS Science and the Earthquake Commission, GEONET, Website, http://www. geonet.org.nz, 2003. 
Gonzalez, W., J. Joselyn, Y. Kamide, H. Kroehl, G. Rostoker, B. Tsurutani, and V. Vasyliunas, What is a geomagnetic storm?, J. Geophys. Res, 99(A4), 5771-5792, 1994.

Green, D., Experimental melting studies on a model upper mantle composition at high pressure under water-saturated and water-undersaturated conditions, Earth and Planetary Science Letters, 19, 37-53, 1973.

Greve, S., and M. Savage, Strong variations in seismic anisotropy across the Hikurangi subduction zone, North Island, New Zealand, In prep, 2007.

Groom, R., and R. Bailey, Decomposition of magnetotelluric impedance tensor in the presence of local 3-D galvanic distortion., J. Geophys. Res., 94, 1913 - 1925, 1989.

Gurrola, H., J. Minster, and T. Owen, The use of velocity spectrum for stacking receiver functions and imaging upper mantle discontinuities, Geophysical Journal International, 117(2), 417-440, 1994.

Haines, A., Seismic wave velocities in the uppermost mantle beneath New Zealand., New Zealand Journal of Geology and Geophysics, 22, 245 - 257, 1979.

Hanks, T., and D. Boore, Moment-magnitude relations in theory and practice, $J$. Geophys. Res., 89, 6229-6235, 1984.

Harrison, T., and R. White, Crustal structure of the Taupo Volcanic Zone, New Zealand: Stretching and igneous intrusion, Geophysical Research Letters, 31(13), L13,615, doi:10.1029/2004GL019885, 2004.

Hatherton, T., Upper mantle inhomogenity beneath New Zealand: surface manifestations., Journal of Geophysical Research, 75, 269 - 284, 1970.

Hauri, E., G. Gaetani, and T. Green, Partitioning of water during melting of the Earth's upper mantle at H2O-undersaturated conditions, Earth and Planetary Science Letters, 248, 715-734, 2006.

Heinz, D., R. Jeanloz, and R. O'Connell, Bulk attenuation in a polycrystalline Earth, Journal of Geophysical Research, 87(B9), 7772-7778, 1982.

Heise, W., T. Caldwell, H. Bibby, and C. Brown, Anisotropy and phase splits in magnetotellurics, Physics of the Earth and Planetary Interiors, 158, 107-121, 2006.

Hirth, G., and D. Kohlstedt, Rheology of the upper mantle and the mantle wedge: A view from the experimentalists, in Inside the subduction factory, Geophysical Monograph, vol. 138, edited by J. Eiler, pp. 83-105, American Geophysical Union, Washington D.C., 2003. 
Hirth, G., and D. L. Kohlstedt, Water in the oceanic upper mantle: implications for rheology, melt extraction and the evolution of the lithosphere, Earth and Planetary Science Letters, 144 (1-2), 93-108, 1996.

Holt, W., and T. Stern, Subduction, platform subsidence, and foreland thrust loading: the late tertiary development of Taranaki Basin, New Zealand, Tectonics, 13(5), 1068-1092, 1994.

Horspool, N., M. K. Savage, and S. Bannister, Implications for intraplate volcanism and back-arc deformation in northwestern New Zealand, from joint inversion of receiver functions and surface waves, Geophysical Journal International, 166(3), 1466-1483, 2006.

Hunt, T., Stokes magnetic anomaly system, New Zealand Journal of Geology and Geophysics, 21, 595-606, 1978.

Ingham, M., K. Whaler, and D. McKnight, Magnetotelluric sounding of the Hikurangi margin New Zealand, Geophysical Journal International, 144, 343-355, 2001.

IRIS PASSCAL, PASSCAL sensor information, Website, http://www.passcal.nmt. edu/instrumentation/Sensor/sensor_info.html, 2005.

Iwamori, H., Transportation of $\mathrm{H} 2 \mathrm{O}$ and melting in subduction zones, Earth and Planetary Science Letters, 160, 65-80, 1998.

Jackson, I., U. Faul, J. Fitz Gerald, and B. Tan, Shear wave attenuation and dispersion in melt-bearing olivine polycrystals: 1 . Specimen fabrication and mechanical testing, J. Geophys. Res., 109, doi:10.1029/2003JB002406, 2004.

Jones, C., and R. A. Phinney, Seismic structure of the lithosphere from teleseismic converted arrivals observed at small arrays in the southern Sierra Nevada and vicinity, California, Journal of Geophysical Research, 103(B5), 10,065-10,090, 1998.

Jones, C. H., H. Kanamori, and S. W. Roecker, Missing roots and mantle "drips": Regional Pn and teleseismic arrival times in the southern Sierra Nevada and vicinity, California, Journal of Geophysical Research, 99(B3), 4567-4602, 1994.

Kamp, P., Tracking crustal processes by FT thermochronology in a forearc high (Hikurangi margin, New Zealand) involving Cretaceous subduction termination and midCenozoic subduction initiation, Tectonophysics, 30\%, 313-343, 1999.

Kamp, P., and K. Furlong, Neogene plate tectonic reconstructions and geodynamics of North Island sedimentary basins: implications for the petroleum systems, in Proceedings 2006 New Zealand Petroleum Conference, 2006. 
Kanamori, H., and D. Anderson, Theoretical basis of some empirical relations in seismology, Bulletin of the Seismological Society of America, 65, 1073-1095, 1975.

Karato, S., Mapping Water Content in the Upper Mantle, in The Subduction Factory, Geophysical Monograph, vol. 138, edited by J. Eiler, pp. 135-152, American Geophysical Union, Washington D.C., 2003.

Karato, S., Remote sensing of hydrogen in Earth's mantle, Reviews in Mineralogy and Geochemistry, 62, 343-375, doi:10.2138/rmg.2006.62.15, 2006.

Karig, D., Kermadec arc - NZ tectonic confluence, New Zealand Journal of Geology and Geophysics, 13, 21-29, 1970.

Kennett, B., E. Engdahl, and R. Buland, Constraints on seismic velocities in the Earth from travel times, Geophys. J. Int., 122, 108-124, 1995.

King, P., Tectonic reconstructions of New Zealand: 40 Ma to the present, New Zealand Journal of Geology and Geophysics, 43, 611-638, 2000.

Kunetz, G., Processing and interpretation of magnetotelluric soundings, Geophysics, 41(1), 96-105, 1976.

Langston, C., Structure under Mount Rainier, Washington, inferred from teleseismic body waves, Journal of Geophysical Research, 84(B9), 4749-4762, 1979.

Lay, T., and T. Wallace, Modern Global Seismology, Academic Press, San Diego, 1995.

Ledo, J., and A. Jones, Upper mantle temperature determined from combining mineral composition, electrical conductivity laboratory studies and magnetotelluric field observation: Application to the intermontane belt, Northern Canadian Cordillera, Earth and Planetary Science Letters, 236, 258-268, 2005.

Lilley, F., Magnetotelluric tesnor decomposition: Part I, theory for a basic procedure, Geophysics, 63(6), 1885-1897, 1998.

Maunder, D. E., New Zealand Seismological Report 2000: Seismological Observatory Bulletin E-183, Tech. rep., Institute of Geological and Nuclear Sciences, 2002.

Maxwell, J. C., A dynamical theory of the electromagnetic field, Phil. Trans. R. Soc. Lond., 155, 459-512, 1865.

McKenzie, D., J. Jackson, and K. Priestley, Thermal structure of oceanicba and continental lithosphere, Earth and Planetary Science Letters, 233, 337-349, 2005.

Molnar, P., and C. Garzione, Bounds on the viscosity coefficient of continental lithosphere from removal of mantle lithosphere beneath the Altiplano and Eastern Cordillera, Tectonics, 26, doi:10.1029/2006TC001964, 2007. 
Molnar, P., and C. Jones, A test of laboratory based rheological parameters of olivine from an analysis of late Cenozoic convective removal of mantle lithoshere beneath the Sierra Nevada, California, USA, Geophysical Journal International, 156, 555$564,2004$.

Molnar, P., B. Tucker, and J. Brune, Corner frequencies of P and S waves and models of earthquake sources, Bull. Seismol. Soc. Am., 63, 2091-2105, 1973.

Mooney, H., Upper mantle inhomogeneity beneath New Zealand: seismic evidence, J. Geophys. Res., 75, 285 - 309, 1970.

Morely, A., G. Stuart, J.-M. Kendall, and M. Reyners, Mantle wedge anisotropy in the Hikurangi subduction zone, central North Island, New Zealand, Geophys. Res. Letters, 33, doi:10.1029/2005GL024569, 2006.

Mortimer, A., A. Tulloch, and T. Ireland, Basement geology of Taranaki and Wanganui basins, New Zealand, N. Z. J. Geology and Geophys., 40, 223-236, 1997.

NOAA, National Geophysical Data Center, Website, http://www.ngdc.noaa.gov, 2007.

Ochs, F., and R. Lange, The density of hydrous magmatic liquids, Science, 283, 13141317, 1999.

Owens, T., and G. Zandt, The response of the continental crust-mantle boundary observed on broadband teleseimic receiver functions, Geophysical Research Letters, 12(10), 705-708, 1985.

Pandey, O., Terrestrial Heat Flow in New Zealand, Ph.d., Victoria University of Wellington, 1981a.

Pandey, O., Terrestrial heat flow in the North Isalnd of New Zealand, Journal of Volcanology and Geothermal Research, 10, 309-316, $1981 \mathrm{b.}$

Park, J., and V. Levin, Receiver functions from multi-taper spectral correlation estimates, Bulletin of the Seismological Society of America, 90, 1507-1520, 2000.

Park, J., C. Lindberg, and F. Vernon, Multitaper spectral analysis of high-frequency seismograms, J. Geophys. Res., 92(B12), 12,675-12,684, 1987.

Park, S., B. Hirasuna, G. Jiracek, and C. Kinn, Magnetotelluric evidence of lithospheric mantle thinning beneath the southern Sierra Nevada, Journal of Geophysical Research, 101(B7), 16,241-16,255, 1996.

Pollack, H., and D. Chapman, On the regional variation of heat flow, geotherms, and lithospheric thickness, Tectonophysics, 38, 279-296, 1977. 
Pulford, A., and T. Stern, Pliocene exhumation and landscape evolution of central North Island: The role of the upper mantle, Journal of Geophysical Research, 109, doi:10.1029/2003JF000046, 2004.

Reilly, W., Horizontal crustal deformation on the Hikurangi Margin, New Zealand Journal of Geology and Geophysics, 33, 393-400, 1990.

Reilly, W., C. Whiteford, and A. Doone, North Island: Gravity map of New Zealand. 1:1000000 Bouguer and isostatic anomalies, Department of Science and Industrial Research, Wellington, New Zealand, 1st ed., 1977.

Resovsky, J., J. Trampert, and R. Van der Hilst, Error bars for the global seismic Q profile, Earth and Planetary Science Letters, 230(413-423), 2005.

Reyners, M., A microearthquake study of the plate boundary, North Island, New Zealand, Ph.D. thesis, Victoria University of Wellington, New Zealand, 1978.

Reyners, M., D. Eberhart-Phillips, G. Stuart, and Y. Nishimura, Imaging subduction from the trench to $300 \mathrm{~km}$ depth beneath the central North Island, New Zealand, with Vp and Vp/Vs, Geophysical Journal International, 165, 565-583, doi:10.1111/ j.1365-246X.2006.02897.x, 2006.

Reyners, M., D. Eberhart-Phillips, and G. Stuart, The role of fluids in lower-crustal earthquakes near continental rifts, Nature, 446, 1075, doi:10.1038/nature05743, 2007.

Risk, G., H. Bibby, and T. Caldwell, DC resistivity mapping with the multiple-source bipole-dipole array in the Central Volcanic Region, New Zealand, J. Geomag. Geoelectr., 45(9), 897-916, 1993.

Romanowicz, B., A global tomographic model of shear attenuation in the upper mantle, Journal of Geophysical Research, 100(B7), 12,375-12,394, 1995.

Roth, E., and D. Wiens, Seismic attenuation tomography of the Tonga-Fiji region using phase pair methods, Journal of Geophysical Research, 104(B3), 4795-4809, 1999.

Satake, K., and T. Hashida, Three-dimensional attenuation structure beneath the North Island, New Zealand, Tectonophysics, 159, 181 - 194, 1989.

Sato, H., I. Sacks, T. Murase, G. Muncill, and H. Fukuyama, Qp - Melting Temperature Relation in Peridotite at High Pressure and Temperature: Attenuation Mechanism and Implications for Mechanical Properties on the Upper Mantle, Journal of Geophysical Research, 94(B8), 10,647-10,661, 1989.

Savage, M. K., Lower Crustal anisotropy or dipping layers? Effects on receiver functions and a case study in New Zealand, Journal of Geophysical Research, 103(no. 7), 15,069-15,089, 1998. 
Schilling, F., G. Partzsch, H. Brasse, and G. Schwarz, Partial melting below the magmatic arc in the central Andes deduced from geoelectromagnetic field experiments and laboratory data., Phys. Earth Planet. Inter., 103, 17-31, 1997.

Schurr, B., A. Reitbrock, G. Asch, R. Kind, and O. Oncken, Evidence for lithospheric detachment in the central Andes from local earthquake tomography, Tectonophysics, 415, 203-223, 2006.

Schutt, D., and C. Lesher, Effects of melt depletion on the density and seismic velocity of garnet Iherzolite, Journal of Geophysical Research, 111, doi:10.1029/ 2003JB002950, 2006.

Sclater, J. G., Heat flow and elevation of the marginal basins of the western Pacific, Journal of Geophysical Research, r7(29), 5705-5719, 1972.

Sherburn, S., and R. White, Crustal seismicity in Taranaki, New Zealand using accurate hypocentres from a dense network, Geophysical Jounral International, 162, 494-506, doi:10.1111/j.1365-246X.2005.02667.x, 2005.

Sheriff, R., and L. Geldart, Exploration Seismology, 2nd ed., Cambridge University Press, 1995.

Shito, A., S. Karato, and J. Park, Frequency dependence of Q in the Earth's upper mantle inferred from continuous spectra of body waves, Geophysical Research Letters, 31, doi:10.1029/2004GL019582, 2004.

Shito, A., S. Karato, K. Matsukage, and Y. Nishihara, Towards mapping the threedimensional distribution of water in the upper mantle from velocity and attenuation tomography, in Earth's deep water cycle, Geophysical Monograph, vol. 168, edited by J. Jacobsen and S. van der Lee, pp. 225-236, American Geophysical Union, 2006.

Simpson, F., and K. Bahr, Practical Magnetotellurics, Cambridge University Press, The Edinburgh Building, Cambridge CB2 2RU, UK, 2005.

St John, D., Parikino-1 exploration well resume, Petroleum Report Series PR452, Shell BP and Todd Oil Services Ltd, 1964.

Stern, T., Regional and residual gravity fields , central North Island, New Zealand., New Zealand Journal of Geology and Geophysics, 22(4), 479 - 485, 1979.

Stern, T., Geophysical Studies of the Upper Crust Within the Central Volcanic Region, New Zealand, The Royal Society of New Zealand Bulletin, 23, 92-111, 1986.

Stern, T., Asymmetric back-arc spreading, heat flux and structure of the Central Volcanic Region of New Zealand, Earth Planet. Sci. Lett., 85, 265 - 276, 1987. 
Stern, T., and F. Davey, A seismic investigation of crustal and upper mantle structure within the Central Volcanic Region of New Zealand, New Zealand Journal of Geology and Geophysics, 30, 217-231, 1987.

Stern, T., E. Smith, F. Davey, and K. Muirhead, Crustal and upper mantle structure of northwestern North Island, New Zealand, from seismic refraction data, Geophysical Journal of the Royal Astronomical Society, 91, 913-936, 1987.

Stern, T., G. Quinlan, and W. Holt, Basin formation behind an active subduction zone: three-dimensional flexural modelling of Wanganui Basin, New Zealand, Basin Research, 4, 197-214, 1992.

Stern, T., W. Stratford, and M. Salmon, Subduction evolution and mantle dynamics at a continetal margin: central North Island, New Zealand, Reviews of Geophysics, 44, doi:10.1026/2005RG000171, 2006.

Stern, T. A., and F. J. Davey, Deep seismic expression of a foreland basin: Taranaki basin, New Zealand, Geology, 18, 979-982, 1990.

Stratford, W., and T. Stern, Strong seismic reflections and melts in the mantle of a continental back-arc basin, Geophysical Research Letters, 31, doi:10.1029/ 2003GL019232, 2004.

Stratford, W., and T. Stern, Crust and upper mantle structure of the continental backarc:central North Island, New Zealand, Geophys. J. Int., 166, 469-484, 2006.

Studt, F., and G. Thompson, Geothermal heat flow in the North Island of New Zealand, New Zealand Journal of Geology and Geophysics, 12, 673-683, 1969.

Takahashi, E., and I. Kushiro, Melting of dry peridotites at high pressures and basalt magma genesis, American Minerolagist, 68, 859-879, 1983.

Takanami, T., I. Sacks, and A. Hasegawa, Attenuation structure beneath the volcanic front in northeastern Japan from broad-band seismograms, Physics of the Earth and Planetary Interiors, 121, 339-357, 2000.

Thurber, C., S. Roecker, K. Roberts, M. Gold, L. Powell, and K. Rittger, Earthquake location and three-dimensional fault zone structure along the creeping section of the San Andreas fault near Parkfield, CA: preparing for SAFOD, Geophysical Research Letters, 30(3), doi:10.1029/2002GL016004, 2003.

Turcotte, D., and G. Schubert, Geodynamics: Applications of continuum physics to geological problems, John Wiley and Sons, New York, 1982.

USGS-NEIC, Global earthquake search, Website, http://neic.usgs.gov/epic/ epic_global.html, 2005. 
Uyeda, S., Some basic problems in the trench-arc-back arc system, in Island arcs, deep sea trenches and back-arc basins, Maurice Ewing Series, vol. 1, edited by M. Talwani and W. C. Pitman, pp. 1-14, 1977.

Uyeda, S., Subduction zones: An introduction to comparative subductology, Tectonophysics, 81, 133-159, 1982.

Uyeda, S., and H. Kanamori, Back-arc opening and the mode of subduction, Journal of Geophysical Research, 84, 1049 - 1061, 1979.

Vernon, F., J. Fletcher, L. Carroll, A. Chave, and E. Sembera, Coherence of seismic body waves from local events as measured by a small-aperture array, Journal of Geophysical Research, 96(B7), 11,981-11,996, 1991.

Wallace, L., J. Beavan, R. McCaffrey, and D. Darby, Subduction zone coupling and tectonic block rotations in the North Island, New Zealand, Journal of Geophysical Research, 109, doi:10.1029/2004JB003241, 2004.

Wang, D., M. Mookherjee, Y. Xu, and S. Karato, The effect of water on electrical conductivity of olivine, Nature, 443, 977-980, 2006.

Wannamaker, P. E., J. A. Stodt, and L. Rijo, A stable finite element solution for twodimensional magnetotelluric modelling, Geophys. J. R. Astron. Soc., 88, 277-296, 1987.

Watson, J., and D. Allen, Compilation of seismic results in Wanganui Basin, Petroleum Report Series PR448, Shell BP and Todd Oil Services Ltd, 1964.

Weaver, J. T., Mathemetical methods for geo-electromagnetic induction, Research Studies Press Ltd., 24 Belvedere Rd, Taunton, Somerset, England TA1 1HD, 1994.

Widmer, R., G. Masters, and F. Gilbert, Spherically symmetric attenuation within the Earth from normal mode data, Geophysical Journal International, 104, 541-553, 1991.

Wiens, D., N. Seama, and J. Conder, Mantle structure and flow patterns beneath active back-arc basins inferred from passive seismic and electromagnetic methods, in Back-arc spreading systems: geological, biological, chamical and physical interactions, Geophysical Monograph, vol. 166, edited by D. Christie, C. Fisher, S. Lee, and S. Givens, pp. 43-62, American Geophysical Union, Washington D.C., 2006.

Wight, D., and F. Bostick, Cascade decimation - a technique for real time estimation of power spectra, Acoustics, Speech, and Signal Processing, IEEE Internation Conference on ICASSP' '80, 5, 626-629, 1980. 
Wilson, C., B. Houghton, M. McWilliams, M. Lanphere, S. Weaver, and R. Briggs, Volcanic and sturctural evolution of Taupo Volcanic Zone, New Zealand: a review, Journal of Volcanology and Geothermal Research, 68, 1-28, 1995.

Zandt, G., The Southern Sierra Nevada Drip and the Mantle Wind Direction, International Geology Review, 45(3), 213-224, 2003.

Zandt, G., and C. Ammon, Continental crust composition constrained by measurements of crustal Poisson's ratio, Nature, 374 (9), 152-154, 1995.

Zheng, Y., T. Lay, M. Flanagan, and Q. Williams, Pervasive seismic wave reflectivity and metasomatism of the Tonga mantle wedge, Science, 316, 855-859, doi:10.1126/ science.1138074, 2007.

Zhu, L., Crustal structure across the San Andreas fault, southern California from teleseismic converted waves, Earth and Planetary Science Letters, 179, 183-190, 2000 .

Zhu, L., and H. Kanamori, Moho depth variation in southern California from teleseismic receiver functions, Journal of Geophysical Research, 105(B2), 2969-2980, 2000. 


\section{Appendix A}

\section{STATION INFORMATION}

Table A.1: Seismic station location and instrumentation.

\begin{tabular}{|c|c|c|c|c|c|c|}
\hline \multirow{5}{*}{ Deployment } & $\begin{array}{c}\text { Station } \\
\text { Name }\end{array}$ & Lat & Long & $\begin{array}{c}\text { Elevation } \\
(\mathrm{m})\end{array}$ & DAS & Seismometer \\
\hline & TUN & -38.96 & 175.36 & 390 & Reftek & L4C-3D \\
& KAI & -39.13 & 175.37 & 680 & Reftek & L4C-3D \\
& ERU & -39.24 & 175.30 & 698 & Reftek & L4C-3D \\
& RAE & -39.41 & 175.25 & 582 & Reftek & L4C-3D \\
& PAP & -39.56 & 175.25 & 364 & Reftek & L4C-3D \\
& SUN & -39.68 & 175.29 & 350 & Reftek & L4C-3D \\
& WRR & -39.82 & 175.15 & 70 & Reftek & L4C-3D \\
\hline CNIPSE & ALL & -38.42 & 175.47 & & EARSS & L4C-3D \\
& PKR & -38.49 & 175.54 & & EARSS & L4C-3D \\
& ARP & -38.15 & 175.66 & & EARSS & L4C-3D \\
& BGL & -38.31 & 175.76 & & EARSS & L4C-3D \\
& PLR & -38.33 & 175.73 & & EARSS & L4C-3D \\
& LLR & -38.04 & 175.86 & & EARSS & L4C-3D \\
& SCHC & -38.43 & 175.73 & 267 & Orion & Guralp CMG-40T \\
& WAIC & -38.37 & 175.65 & 350 & Orion & Guralp CMG-40T \\
& YUPC & -38.32 & 175.55 & 607 & Orion & Guralp CMG-40T \\
& MILC & -38.26 & 175.42 & 273 & Orion & Guralp CMG-40T \\
\hline WAZ & -39.76 & 174.99 & 382 & & Guralp CMG-3ESP \\
& VRZ & -39.13 & 174.76 & 166 & & Guralp CMG-3ESP \\
& HIZ & -38.51 & 174.86 & 237 & & Streckheisen STS-2 \\
\hline \multirow{7}{*}{ GEONET }
\end{tabular}


Table A.2: Seismic station deployment periods for TRAP and CNIPSE arrays.

\begin{tabular}{|c|c|c|c|}
\hline \multirow{1}{*}{ Deployment } & $\begin{array}{c}\text { Station } \\
\text { Name }\end{array}$ & Start date & End date \\
\hline TRAP & TUN & $12 / 02 / 2003$ & $24 / 03 / 2003$ \\
& KAI & $18 / 06 / 2002$ & $14 / 04 / 2003$ \\
& ERU & $21 / 08 / 2002$ & $13 / 04 / 2003$ \\
& RAE & $18 / 06 / 2002$ & $14 / 04 / 2003$ \\
& PAP & $19 / 06 / 2002$ & $13 / 04 / 2003$ \\
& SUN & $20 / 08 / 2002$ & $13 / 04 / 2003$ \\
& WRR & $19 / 06 / 2002$ & $13 / 02 / 2003$ \\
\hline CNIPSE & ALL & $15 / 02 / 2001$ & $04 / 07 / 2001$ \\
& PKR & $08 / 02 / 2001$ & $26 / 05 / 2001$ \\
& ARP & $19 / 01 / 2001$ & $05 / 07 / 2001$ \\
& BGL & $18 / 01 / 2001$ & $05 / 07 / 2001$ \\
& PLR & $08 / 03 / 2001$ & $05 / 07 / 2001$ \\
& LLR & $18 / 01 / 2001$ & $05 / 07 / 2001$ \\
& SCHC & $10 / 01 / 2001$ & $02 / 07 / 2001$ \\
& WAIC & $12 / 01 / 2001$ & $02 / 07 / 2001$ \\
& YUPC & $12 / 01 / 2001$ & $11 / 12 / 2001$ \\
& MILC & $04 / 07 / 2001$ & $12 / 12 / 2001$ \\
\hline
\end{tabular}

Table A.3: Magnetotelluric station locations

\begin{tabular}{|c|c|c|c|}
\hline $\begin{array}{c}\text { Station } \\
\text { Name }\end{array}$ & Latitude & Longitude & Elevation $(\mathrm{m})$ \\
\hline 001 & -38.99 & 175.24 & 358 \\
002 & -39.14 & 175.14 & 176 \\
003 & -39.31 & 175.20 & 253 \\
004 & -39.50 & 175.22 & 381 \\
005 & -39.62 & 175.38 & 388 \\
006 & -39.85 & 175.18 & 40 \\
\hline
\end{tabular}




\section{Appendix B}

\section{RECEIVER FUNCTIONS}

Table B.1: Events used in receiver functions for the TRAP array. Distance in degrees (Dist) and backazimuth (BAZ) are calculated from station RAE

\begin{tabular}{|r|c|c|r|r|r|r|r|r|}
\hline Year & Day & Time & Lat & Long & Magnitude & Depth & Dist & BAZ \\
\hline 2002 & 169 & $13: 56: 22.83$ & -30.81 & -71.12 & 6.6 & 54 & 87 & 128 \\
2002 & 172 & $00: 05: 44.85$ & -4.49 & 146.77 & 6.0 & 33 & 43 & 316 \\
2002 & 178 & $05: 50: 35.11$ & -6.96 & 104.18 & 6.9 & 11 & 71 & 277 \\
2002 & 178 & $07: 16: 10.37$ & -13.27 & 167.05 & 6.0 & 186 & 27 & 342 \\
2002 & 179 & $17: 19: 30.27$ & 43.75 & 130.67 & 7.3 & 566 & 92 & 329 \\
2002 & 180 & $02: 39: 00.72$ & -12.40 & 166.52 & 6.2 & 33 & 28 & 341 \\
2002 & 181 & $21: 29: 36.30$ & -22.20 & 179.25 & 6.5 & 620 & 17 & 12 \\
2002 & 184 & $23: 00: 18.47$ & -5.03 & 147.34 & 6.2 & 31 & 43 & 316 \\
2002 & 250 & $08: 14: 19.65$ & -20.27 & -176.04 & 6.0 & 209 & 20 & 24 \\
2002 & 251 & $13: 15: 55.72$ & -22.84 & 178.93 & 6.0 & 618 & 17 & 12 \\
2002 & 251 & $18: 44: 23.71$ & -3.30 & 142.95 & 7.8 & 13 & 46 & 313 \\
2002 & 256 & $22: 28: 29.46$ & 13.04 & 93.07 & 6.7 & 21 & 92 & 285 \\
2002 & 258 & $08: 39: 32.70$ & 44.83 & 129.92 & 6.4 & 586 & 93 & 330 \\
2002 & 259 & $13: 23: 00.99$ & -3.31 & 142.68 & 6.3 & 10 & 47 & 312 \\
2002 & 260 & $11: 20: 23.39$ & -3.28 & 142.77 & 6.0 & 10 & 46 & 312 \\
2002 & 263 & $13: 33: 41.62$ & -1.74 & 134.01 & 6.0 & 10 & 53 & 304 \\
2002 & 263 & $15: 43: 35.46$ & -1.68 & 134.23 & 6.4 & 10 & 53 & 305 \\
2002 & 267 & $22: 54: 21.48$ & -10.56 & 161.11 & 6.3 & 10 & 31 & 333 \\
2002 & 267 & $03: 57: 22.28$ & -31.52 & -69.2 & 6.3 & 119 & 87 & 130 \\
2002 & 267 & $04: 13: 11.63$ & -10.53 & 161.2 & 6.2 & 10 & 31 & 333 \\
2002 & 267 & $23: 01: 28.67$ & -10.65 & 161.21 & 6.5 & 19 & 31 & 333 \\
2002 & 277 & $19: 05: 48.77$ & -20.99 & -179.02 & 6.3 & 621 & 19 & 17 \\
2002 & 279 & $15: 46: 33.01$ & -8.20 & 118.34 & 6.3 & 10 & 59 & 286 \\
2002 & 281 & $01: 23: 58.80$ & -42.04 & 88.38 & 6.0 & 10 & 63 & 237 \\
2002 & 283 & $10: 50: 20.57$ & -1.76 & 134.30 & 7.7 & 10 & 53 & 305 \\
2002 & 283 & $12: 28: 25.80$ & -1.51 & 133.97 & 6.7 & 10 & 53 & 305 \\
2002 & 283 & $21: 19: 58.51$ & -1.48 & 134.11 & 6.0 & 10 & 53 & 305 \\
2002 & 285 & $20: 09: 11.46$ & -8.30 & -71.74 & 6.9 & 534 & 102 & 111 \\
2002 & 285 & $23: 43: 12.85$ & 15.10 & 118.50 & 6.1 & 33 & 76 & 304 \\
2002 & 286 & $20: 55: 07.46$ & -14.60 & -175.42 & 6.1 & 10 & 26 & 21 \\
2002 & 287 & $14: 12: 43.75$ & 41.17 & 142.25 & 6.1 & 61 & 86 & 336 \\
\hline & & & & & continued on next page \\
\end{tabular}


Table B.1 - continued

\begin{tabular}{|c|c|c|c|c|c|c|c|c|}
\hline Year & Day & Time & Lat & $\overline{\text { Long }}$ & Magnitude & Depth & Dist & BAZ \\
\hline 2002 & 289 & $14: 13: 12.74$ & -15.68 & -173.05 & 6.0 & 33 & 26 & 27 \\
\hline 2002 & 290 & $04: 23: 55.94$ & -19.84 & -178.40 & 6.2 & 627 & 20 & 17 \\
\hline 2002 & 290 & $17: 52: 44.19$ & -3.60 & 140.23 & 6.3 & 33 & 48 & 309 \\
\hline 2002 & 292 & 12:09:05.38 & 44.30 & 149.96 & 6.3 & 33 & 86 & 342 \\
\hline 2002 & 295 & $11: 39: 04.21$ & -20.63 & -178.39 & 6.2 & 549 & 20 & 18 \\
\hline 2002 & 297 & 21.53:43.19 & 6.03 & 94.42 & 6.2 & 64 & 86 & 280 \\
\hline 2002 & 304 & $01: 35: 16.67$ & -3.44 & 148.64 & 6.1 & 10 & 43 & 319 \\
\hline 2002 & 306 & $01: 26: 10.70$ & 2.82 & 96.08 & 7.6 & 30 & 83 & 276 \\
\hline 2002 & 306 & $09: 46: 46.70$ & 2.95 & 96.39 & 6.4 & 27 & 83 & 279 \\
\hline 2002 & 307 & $03: 37: 42.07$ & 38.89 & 141.98 & 6.4 & 39 & 83 & 334 \\
\hline 2002 & 316 & 01:46:48.94 & -56.55 & -27.54 & 6.2 & 120 & 82 & 168 \\
\hline 2002 & 319 & 19:58:31.78 & -56.05 & -36.40 & 6.6 & 10 & 81 & 163 \\
\hline 2002 & 321 & $04: 53: 53.54$ & 47.82 & 146.21 & 7.3 & 459 & 91 & 341 \\
\hline 2002 & 330 & $00: 48: 15.04$ & 51.47 & -173.54 & 6.1 & 20 & 91 & 7 \\
\hline 2002 & 344 & 01:28:33.67 & -50.03 & -114.12 & 6.0 & 10 & 50 & 127 \\
\hline 2002 & 344 & $04: 27: 54.60$ & -24.14 & 179.24 & 6.1 & 530 & 16 & 14 \\
\hline 2002 & 345 & 03:49:40.19 & -3.81 & 135.12 & 6.3 & 10 & 51 & 304 \\
\hline 2002 & 346 & 08:30:42.77 & -4.79 & 153.27 & 6.7 & 34 & 40 & 324 \\
\hline 2002 & 351 & $04: 32: 53.07$ & -56.95 & -24.83 & 6.3 & 10 & 83 & 169 \\
\hline 2002 & 352 & $14: 12: 21.75$ & -57.09 & -24.98 & 6.1 & 10 & 82 & 169 \\
\hline 2002 & 354 & $14: 14: 42.05$ & -3.08 & 147.94 & 6.4 & 33 & 44 & 319 \\
\hline 2002 & 364 & 04:49:08.69 & 7.47 & 123.41 & 6.3 & 10 & 67 & 302 \\
\hline 2003 & 004 & $05: 15: 03.84$ & -20.57 & -177.66 & 6.5 & 378 & 20 & 20 \\
\hline 2003 & 007 & $00: 54: 51.56$ & -33.76 & -70.05 & 6.0 & 110 & 85 & 131 \\
\hline 2003 & 009 & $02: 50: 45.79$ & -19.66 & -176.29 & 6.0 & 10 & 21 & 23 \\
\hline 2003 & 010 & $13: 11: 56.91$ & -5.31 & 153.70 & 6.7 & 71 & 39 & 325 \\
\hline 2003 & 020 & 08:43:06.07 & -10.49 & 160.77 & 7.8 & 33 & 32 & 332 \\
\hline 2003 & 022 & 02:06:34.61 & 18.77 & -104.10 & 7.6 & 24 & 95 & 70 \\
\hline 2003 & 027 & $17: 56: 25.83$ & -46.05 & 35.06 & 6.4 & 10 & 88 & 207 \\
\hline 2003 & 041 & 04:49:31.12 & -6.01 & 149.79 & 6.3 & 33 & 40 & 319 \\
\hline 2003 & 043 & $22: 33: 30.83$ & -3.65 & 144.24 & 6.2 & 10 & 45 & 314 \\
\hline 2003 & 046 & 11:01:59.86 & 12.17 & 124.08 & 6.3 & 10 & 70 & 306 \\
\hline 2003 & 050 & 05:01:40.00 & 44.09 & 141.76 & 6.7 & 219 & 88 & 337 \\
\hline 2003 & 051 & $10: 37: 06.00$ & -0.96 & 126.75 & 6.0 & 43 & 58 & 298 \\
\hline 2003 & 060 & $06: 12: 47.40$ & -1.70 & 134.31 & 6.0 & 28 & 53 & 305 \\
\hline 2003 & 068 & $10: 36: 23.03$ & -6.82 & 130.99 & 6.0 & 45 & 51 & 297 \\
\hline 2003 & 069 & 02:09:37.08 & 1.69 & 127.30 & 6.4 & 93 & 60 & 301 \\
\hline 2003 & 070 & $07: 27: 32.65$ & -4.69 & 153.24 & 6.8 & 40 & 40 & 324 \\
\hline 2003 & 070 & $07: 35: 42.00$ & -4.67 & 153.18 & 6.1 & 34 & 40 & 324 \\
\hline 2003 & 073 & 07:06:13.31 & -0.41 & 132.95 & 6.3 & 33 & 55 & 304 \\
\hline 2003 & 073 & $12: 54: 12.12$ & -17.42 & -175.18 & 6.4 & 274 & 23 & 24 \\
\hline 2003 & 076 & $16: 36: 17.31$ & 51.27 & 177.98 & 7.1 & 33 & 90 & 2 \\
\hline 2003 & 078 & 00:03:42.92 & -9.39 & 156.59 & 6.2 & 33 & 34 & 326 \\
\hline 2003 & 084 & $02: 53: 25.03$ & -8.29 & 120.74 & 6.5 & 33 & 58 & 287 \\
\hline 2003 & 085 & $04: 22: 29.00$ & 12.55 & 92.61 & 6.0 & 30 & 92 & 284 \\
\hline 2003 & 087 & $17: 31: 47.77$ & -15.32 & -173.51 & 6.2 & 41 & 26 & 25 \\
\hline 2003 & 089 & 18:13:34.09 & -3.17 & 127.54 & 6.2 & 33 & 56 & 297 \\
\hline
\end{tabular}


Table B.1 - continued

\begin{tabular}{|r|c|c|r|r|r|r|r|r|}
\hline Year & Day & Time & Lat & Long & Magnitude & Depth & Dist & BAZ \\
\hline 2003 & 090 & $01: 06: 53.05$ & -6.18 & 151.43 & 6.2 & 46 & 39 & 321 \\
\hline
\end{tabular}

Table B.2: Events used in receiver functions for the GEONET stations. Distance in degrees (Dist) and backazimuth (BAZ) are calculated from station VRZ.

\begin{tabular}{|c|c|c|c|c|c|c|c|c|}
\hline Year & Day & Time & Lat & Long & Magnitude & Depth & Dist & BAZ \\
\hline 2004 & 11 & $04: 32: 47.79$ & -36.70 & 53.35 & 6.20 & 5 & 87 & 223 \\
\hline 2004 & 28 & $22: 15: 30.70$ & -3.12 & 127.40 & 6.70 & 17 & 56 & 298 \\
\hline 2004 & 29 & $03: 52: 52.30$ & -50.21 & -114.78 & 6.10 & 10 & 50 & 127 \\
\hline 2004 & 36 & $21: 05: 02.84$ & -3.62 & 135.54 & 7.10 & 16 & 50 & 305 \\
\hline 2004 & 38 & 02:42:35.21 & -4.00 & 135.02 & 7.50 & 10 & 50 & 304 \\
\hline 2004 & 39 & 08:58:51.80 & -3.66 & 135.34 & 6.90 & 25 & 50 & 304 \\
\hline 2004 & 51 & 05:58:45.19 & -11.61 & 166.45 & 6.00 & 84 & 28 & 343 \\
\hline 2004 & 53 & $06: 46: 27.04$ & -1.56 & 100.49 & 6.30 & 42 & 77 & 279 \\
\hline 2004 & 57 & $22: 58: 41.38$ & -53.16 & 159.65 & 6.00 & 10 & 17 & 212 \\
\hline 2004 & 72 & $22: 13: 13.86$ & -15.58 & -175.10 & 6.00 & 271 & 25 & 24 \\
\hline 2004 & 74 & $16: 30: 42.32$ & -17.27 & -172.32 & 6.10 & 12 & 25 & 31 \\
\hline 2004 & 78 & 20:04:24.83 & -23.76 & -176.00 & 6.20 & 10 & 17 & 30 \\
\hline 2004 & 94 & 23:02:00.87 & 36.43 & 141.01 & 6.00 & 31 & 81 & 333 \\
\hline 2004 & 96 & $20: 34: 21.93$ & -20.44 & -173.94 & 6.20 & 8 & 21 & 31 \\
\hline 2004 & 99 & $04: 57: 50.48$ & -36.26 & -97.83 & 6.40 & 10 & 67 & 118 \\
\hline 2004 & 100 & $15: 23: 35.05$ & -13.17 & 167.20 & 6.50 & 228 & 27 & 343 \\
\hline 2004 & 102 & $07: 37: 29.85$ & -3.73 & 140.08 & 6.20 & 20 & 47 & 309 \\
\hline 2004 & 102 & $18: 06: 12.48$ & 42.92 & 144.84 & 6.10 & 41 & 86 & 338 \\
\hline 2004 & 105 & 01:33:05.30 & -17.85 & -174.56 & 6.00 & 143 & 23 & 27 \\
\hline 2004 & 106 & 20:06:55.30 & -19.37 & 169.63 & 6.30 & 10 & 20 & 346 \\
\hline 2004 & 107 & $16: 58: 36.94$ & -24.52 & -175.88 & 6.00 & 10 & 17 & 31 \\
\hline 2004 & 107 & $21: 57: 05.41$ & -5.21 & 102.72 & 6.00 & 44 & 73 & 277 \\
\hline 2004 & 108 & $15: 58: 24.61$ & -7.35 & 128.37 & 6.10 & 128 & 52 & 295 \\
\hline 2004 & 113 & 10:11:12.66 & -17.59 & 167.89 & 6.00 & 10 & 22 & 342 \\
\hline 2004 & 113 & $14: 16: 07$ & -3.36 & 146.85 & 6.00 & 35 & 44 & 317 \\
\hline 2004 & 114 & $01: 50: 30.22$ & -9.36 & 122.84 & 6.70 & 65 & 55 & 288 \\
\hline 2004 & 115 & 07:44:11.30 & -21.90 & -174.82 & 6.10 & 8 & 19 & 30 \\
\hline 2004 & 118 & $23: 28: 18.88$ & -17.76 & 167.76 & 6.00 & 10 & 22 & 342 \\
\hline 2004 & 124 & $04: 36: 50.04$ & -37.69 & -73.41 & 6.60 & 21 & 81 & 131 \\
\hline 2004 & 128 & $01: 26: 42.20$ & -21.99 & 170.28 & 6.30 & 14 & 18 & 346 \\
\hline 2004 & 132 & $08: 28: 48.28$ & 0.41 & 97.82 & 6.20 & 21 & 80 & 279 \\
\hline 2004 & 134 & $09: 58: 43.45$ & -3.58 & 150.73 & 6.40 & 10 & 42 & 322 \\
\hline 2004 & 140 & 07:04:11.71 & 22.66 & 121.50 & 6.20 & 20 & 79 & 311 \\
\hline 2004 & 161 & 22:52:08.80 & -51.60 & 139.62 & 6.40 & 10 & 27 & 231 \\
\hline 2004 & 162 & $15: 19: 57.75$ & 55.68 & 160.00 & 6.90 & 188 & 95 & 352 \\
\hline 2004 & 167 & $11: 16: 31.50$ & -38.85 & -73.15 & 6.10 & 37 & 81 & 133 \\
\hline 2004 & 177 & 02:35:07.79 & -6.71 & 130.38 & 6.10 & 70 & 51 & 297 \\
\hline
\end{tabular}


Table B.2 - continued

\begin{tabular}{|c|c|c|c|c|c|c|c|c|}
\hline Year & Day & Time & Lat & Long & "Magnitude & Depth & Dist & BAZ \\
\hline 2004 & 182 & $23: 37: 25.45$ & 0.80 & 124.73 & 6.30 & 90 & 61 & 298 \\
\hline 2004 & 190 & $10: 30: 49.16$ & 47.20 & 151.30 & 6.40 & 128 & 88 & 344 \\
\hline 2004 & 190 & $19: 54: 33.84$ & -25.06 & -115.96 & 6.00 & 10 & 59 & 98 \\
\hline 2004 & 193 & $23: 46: 12.56$ & -20.25 & -126.93 & 6.10 & 12 & 53. & 87 \\
\hline 2004 & 197 & $04: 27: 14.73$ & -17.66 & -178.76 & 7.10 & 565 & 22 & 17 \\
\hline 2004 & 204 & $09: 45: 14.90$ & 26.49 & 128.89 & 6.10 & 20 & 78 & 319 \\
\hline 2004 & 207 & 14:35:19.06 & -2.43 & 103.98 & 7.30 & 582 & 74 & 280 \\
\hline 2004 & 210 & $03: 56: 28.60$ & -0.44 & 133.09 & 6.50 & 13 & 54 & 305 \\
\hline 2004 & 214 & 19:03:10.29 & -63.56 & -166.67 & 6.00 & 10 & 27 & 162 \\
\hline 2004 & 241 & $13: 41: 25.60$ & -35.17 & -70.53 & 6.50 & 5 & 85 & 132 \\
\hline 2004 & 247 & 19:04:47.98 & -15.25 & -173.34 & 6.20 & 10 & 26 & 27 \\
\hline 2004 & 249 & 10:07:07.82 & 33.07 & 136.62 & 7.20 & 14 & 80 & 328 \\
\hline 2004 & 249 & $14: 57: 18.61$ & 33.18 & 137.07 & 7.40 & 10 & 80 & 329 \\
\hline 2004 & 250 & $12: 42: 59.39$ & -55.37 & -28.98 & 6.90 & 10 & 84 & 167 \\
\hline 2004 & 250 & 23:29:35.09 & 33.21 & 137.23 & 6.60 & 10 & 80 & 329 \\
\hline 2004 & 251 & 11:53:06.11 & -28.57 & -65.84 & 6.40 & 22 & 92 & 130 \\
\hline 2004 & 252 & $14: 58: 25.83$ & 33.14 & 137.20 & 6.20 & 21 & 80 & 329 \\
\hline 2004 & 255 & $21: 52: 38.30$ & -57.98 & -25.34 & 6.10 & 63 & 82 & 169 \\
\hline 2004 & 257 & 03:00:12.85 & 44.00 & 151.41 & 6.10 & 8 & 85 & 343 \\
\hline 2004 & 259 & $19: 10: 50.60$ & 14.22 & 120.41 & 6.00 & 115 & 73 & 305 \\
\hline 2004 & 263 & 20:26:04.10 & 52.21 & 174.03 & 6.20 & 25 & 91 & 360 \\
\hline 2004 & 272 & $15: 29: 53.82$ & -52.51 & 28.02 & 6.40 & 10 & 84 & 200 \\
\hline 2004 & 278 & $19: 20: 34.98$ & 14.55 & 146.99 & 6.00 & 7 & 59 & 328 \\
\hline 2004 & 280 & $22: 30: 56.95$ & -0.67 & 134.43 & 6.20 & 10 & 53 & 306 \\
\hline 2004 & 282 & $08: 27: 53.54$ & -10.95 & 162.16 & 6.90 & 36 & 30 & 335 \\
\hline 2004 & 282 & 14:36:06.11 & 13.93 & 120.53 & 6.50 & 105 & 73 & 305 \\
\hline 2004 & 289 & 04:08:50.24 & 24.53 & 122.69 & 6.70 & 94 & 80 & 313. \\
\hline 2004 & 294 & $18: 32: 24.57$ & -14.03 & 166.61 & 6.10 & 59 & 26 & 342 \\
\hline 2004 & 297 & 08:56:00.86 & 37.23 & 138.78 & 6.60 & 16 & 83 & 332 \\
\hline 2004 & 297 & 09:34:04.99 & 37.32 & 138.81 & 6.30 & 10 & 83 & 332 \\
\hline 2004 & 298 & $21: 04: 57.06$ & 37.31 & 138.70 & 6.00 & 11 & 83 & 332 \\
\hline 2004 & 300 & $22: 53: 07.86$ & -57.07 & -24.68 & 6.40 & 10 & 83 & 169 \\
\hline 2004 & 301 & $01: 40: 50.26$ & 37.28 & 138.88 & 6.00 & 14 & 83 & 332 \\
\hline 2004 & 308 & 08:31:43.73 & 14.47 & 146.84 & 6.00 & 10 & 59 & 328 \\
\hline 2004 & 310 & $05: 18: 35.05$ & -4.36 & 143.93 & 6.00 & 125 & 44 & 313 \\
\hline 2004 & 312 & 02:02:26.17 & 47.95 & 144.48 & 6.20 & 474 & 91 & 340 \\
\hline 2004 & 313 & $15: 55: 01.15$ & 24.10 & 122.54 & 6.30 & 29 & 80 & 313 \\
\hline 2004 & 316 & 10:02:47.33 & 42.14 & 144.34 & 6.10 & 32 & 85 & 338 \\
\hline 2004 & 316 & $17: 34: 52.05$ & -11.13 & 162.21 & 6.70 & 10 & 30 & 335 \\
\hline 2004 & 316 & $21: 26: 41.15$ & -8.15 & 124.87 & 7.50 & 10 & 54 & 291 \\
\hline 2004 & 316 & $22: 49: 48.14$ & -8.26 & 124.93 & 6.40 & 10 & 54 & 291 \\
\hline 2004 & 317 & $06: 36: 16.76$ & -26.70 & -63.32 & 6.10 & 568 & 95 & 130 \\
\hline 2004 & 322 & 21:09:13.13 & -20.07 & -178.71 & 6.60 & 622 & 20 & 18 \\
\hline 2004 & 326 & $11: 07: 13.65$ & -15.40 & -174.91 & 6.00 & 256 & 25 & 24 \\
\hline 2004 & 327 & $20: 26: 23.90$ & -46.68 & 164.72 & 7.10 & 10 & 11 & 221 \\
\hline 2004 & 331 & 02:25:03.31 & -3.61 & 135.40 & 7.20 & 10 & 50 & 305 \\
\hline 2004 & 333 & $02: 35: 13.41$ & -26.52 & -113.83 & 6.60 & 10 & 60 & 101 \\
\hline
\end{tabular}


Table B. 2 - continued

\begin{tabular}{|c|c|c|c|c|c|c|c|c|}
\hline Year & Day & Time & Lat & $\overline{\text { Long }}$ & Magnitude & Depth & Dist & BAZ \\
\hline 2004 & 333 & $07: 36: 45.40$ & -3.64 & 135.45 & 6.20 & 23 & 50 & 305 \\
\hline 2004 & 333 & $18: 32: 14.13$ & 43.01 & 145.12 & 7.00 & 39 & 86 & 339 \\
\hline 2004 & 341 & $14: 15: 11.89$ & 42.90 & 145.23 & 6.80 & 35 & 86 & 339 \\
\hline 2004 & 341 & $16: 26: 28.96$ & -18.48 & -178.08 & 6.10 & 439 & 21 & 19 \\
\hline 2004 & 358 & 14:59:04.41 & -49.31 & 161.35 & 8.10 & 10 & 14 & 219 \\
\hline 2004 & 361 & $00: 58: 53.45$ & 3.30 & 95.98 & 9.00 & 30 & 83 & 280 \\
\hline 2004 & 361 & $04: 21: 29.81$ & 6.91 & 92.96 & 7.50 & 39 & 88 & 280 \\
\hline 2004 & 361 & 09:20:01.61 & 8.88 & 92.38 & 6.60 & 16 & 90 & 282 \\
\hline 2004 & 361 & 10:19:31.73 & 13.46 & 92.74 & 6.30 & 26 & 92 & 285 \\
\hline 2004 & 361 & 11:05:00.72 & 13.53 & 92.84 & 6.30 & 13 & 92 & 285 \\
\hline 2004 & 362 & $00: 32: 16.48$ & 5.48 & 94.47 & 6.10 & 33 & 86 & 280 \\
\hline 2004 & 362 & $00: 49: 28.59$ & 12.98 & 92.39 & 6.10 & 23 & 92 & 285 \\
\hline 2004 & 362 & 09:39:06.80 & 5.35 & 94.65 & 6.20 & 35 & 860 & 280 \\
\hline 2004 & 364 & $01: 50: 52.57$ & 9.11 & 93.76 & 6.10 & 8 & 89 & 283 \\
\hline 2004 & 364 & $05: 56: 47.54$ & 8.79 & 93.20 & 6.20 & 12 & 89 & 282 \\
\hline 2004 & 366 & $02: 24: 00.52$ & 7.12 & 92.53 & 6.30 & 14 & 88 & 280 \\
\hline 2004 & 366 & $12: 04: 57.52$ & 6.20 & 92.91 & 6.10 & 11 & 88 & 280 \\
\hline 2005 & 01 & $06: 25: 44.82$ & 5.10 & 92.30 & 6.70 & 11 & 87 & 279 \\
\hline 2005 & 01 & $: 08: 07.80$ & 7.34 & 94.46 & 6.10 & 55 & 87 & 282 \\
\hline 2005 & 02 & $15: 35: 56.72$ & 6.36 & 92.79 & 6.40 & 30 & 88 & 280 \\
\hline 2005 & 03 & $17: 59: 28.89$ & -50.66 & 161.87 & 6.10 & 10 & 15 & 214 \\
\hline 2005 & 06 & $00: 56: 29.91$ & 5.32 & 94.83 & 6.10 & 49 & 86 & 280 \\
\hline 2005 & 08 & 18:45:03.82 & -55.30 & -27.96 & 6.00 & 10 & 84 & 167 \\
\hline 2005 & 09 & $22: 12: 56.51$ & 4.93 & 95.11 & 6.10 & 40 & 85 & 280 \\
\hline 2005 & 14 & $8: 33: 14.46$ & -4.24 & 152.72 & 6.10 & 10 & 40 & 324 \\
\hline 2005 & 15 & $: 47: 05.13$ & -6.46 & 105.24 & 6.00 & 58 & 70 & 278 \\
\hline 2005 & 16 & $08: 25: 04.47$ & -25.53 & -176.30 & 6.20 & 16 & 16 & 32 \\
\hline 2005 & 16 & $20: 17: 52.76$ & 10.93 & 140.84 & 6.70 & 24 & 59 & 320 \\
\hline 2005 & 17 & $10: 50: 32.56$ & 10.99 & 140.68 & 6.10 & 12 & 59 & 320 \\
\hline 2005 & 18 & 14:09:06.22 & 42.95 & 144.87 & 6.30 & 42 & 86 & 338 \\
\hline 2005 & 19 & $06: 11: 36.40$ & 34.06 & 141.49 & 6.60 & 27 & 79 & 332 \\
\hline 2005 & 22 & $20: 30: 17.35$ & -7.73 & 159.48 & 6.50 & 29 & 34 & 332 \\
\hline 2005 & 23 & $20: 10: 12.15$ & -1.20 & 119.93 & 6.30 & 11 & 63 & 293 \\
\hline 2005 & 24 & $04: 16: 47.44$ & 7.33 & 92.48 & 6.30 & 30 & 89 & 281 \\
\hline 2005 & 26 & $22: 00: 42.57$ & 2.70 & 94.60 & 6.20 & 22 & 84 & 278 \\
\hline 2005 & 33 & 02:30:25.94 & 14.08 & 144.71 & 6.30 & 158 & 60 & 326 \\
\hline 2005 & 36 & $03: 34: 25.73$ & 16.01 & 145.87 & 6.60 & 142 & 61 & 328 \\
\hline 2005 & 36 & 04:03:13.84 & 2.26 & 94.99 & 6.00 & 30 & 83 & 2780 \\
\hline 2005 & 36 & $12: 23: 18.94$ & 5.29 & 123.34 & 7.10 & 525 & 65 & 301 \\
\hline 2005 & 38 & 20:02:17.69 & -4.53 & 153.19 & 6.10 & 36 & 40 & 325 \\
\hline 2005 & 39 & $14: 48: 21.97$ & -14.25 & 167.26 & 6.70 & 206 & 26 & 343 \\
\hline 2005 & 40 & $13: 27: 25.34$ & 4.80 & 95.12 & 6.00 & 44 & 85 & 280 \\
\hline 2005 & 40 & 18:46:09.97 & 26.09 & 144.00 & 6.30 & 24 & 71 & 331 \\
\hline 2005 & 41 & $16: 53: 19.99$ & -23.10 & 169.22 & 6.30 & 9 & 17 & 342 \\
\hline 2005 & 45 & $17: 06: 52.64$ & -0.13 & 98.73 & 6.00 & 47 & 79 & 279 \\
\hline 2005 & 46 & $14: 42: 25.85$ & 4.76 & 126.42 & 6.50 & 39 & 62 & 303 \\
\hline 2005 & 50 & 00:04:43.59 & -5.56 & 122.13 & 6.50 & 10 & 58 & 291 \\
\hline
\end{tabular}


Table B.2 - continued

\begin{tabular}{|c|c|c|c|c|c|c|c|c|}
\hline Year & Day & Time & Lat & Long & Magnitude & Depth & Dist & BAZ \\
\hline 2005 & 54 & 11:33:52.90 & -6.24 & 150.66 & 6.00 & 10 & 39 & 320 \\
\hline 2005 & 57 & $12: 56: 52.62$ & 2.91 & 95.59 & 6.80 & 36 & 83 & 279 \\
\hline 2005 & 61 & $10: 42: 12.23$ & -6.53 & 129.93 & 7.10 & 201 & 52 & 297 \\
\hline 2005 & 63 & 19:05:19.89 & 2.67 & 126.41 & 6.10 & 59 & 61 & 301 \\
\hline 2005 & 65 & 04:39:59.43 & -11.05 & 163.04 & 6.10 & 10 & 30 & 336 \\
\hline 2005 & 78 & $15: 02: 42.67$ & -20.41 & -174.34 & 6.10 & 18 & 21 & 30 \\
\hline 2005 & 78 & $17: 34: 46.18$ & -21.89 & -179.55 & 6.30 & 598 & 183 & 17 \\
\hline 2005 & 79 & 01:53:41.83 & 33.81 & 130.13 & 6.70 & 10 & 84 & 324 \\
\hline 2005 & 80 & 12:23:54.09 & -24.98 & -63.47 & 6.90 & 579 & 96 & 129 \\
\hline 2005 & 80 & $12: 43: 12.36$ & -24.73 & -63.51 & 6.40 & 570 & 96 & 129 \\
\hline 2005 & 85 & $15: 40: 34.53$ & -4.89 & 129.94 & 6.10 & 10 & 53 & 298 \\
\hline 2005 & 87 & 16:09:36.53 & 2.09 & 97.11 & 8.60 & 30 & 82 & 279 \\
\hline 2005 & 87 & $18: 30: 44.56$ & 0.92 & 97.87 & 6.10 & 36 & 80 & 279 \\
\hline 2005 & 89 & $16: 19: 41.10$ & 2.99 & 95.41 & 6.30 & 22 & 84 & 279 \\
\hline 2005 & 89 & $17: 41: 57.16$ & -22.46 & -179.75 & 6.10 & 588 & 17 & 17 \\
\hline 2005 & 90 & 01:46:17.76 & -18.45 & 175.99 & 6.00 & 10 & 21 & 3 \\
\hline 2005 & 93 & $00: 59: 21.42$ & 0.37 & 98.32 & 6.00 & 30 & 80 & 279 \\
\hline 2005 & 93 & 03:10:56.47 & 2.02 & 97.94 & 6.30 & 36 & 81 & 280 \\
\hline 2005 & 97 & 19:43:14.92 & 2.97 & 126.48 & 6.00 & 59 & 61 & 302 \\
\hline 2005 & 98 & $05: 48: 37.88$ & -0.22 & 97.73 & 6.30 & 20 & 80 & 278 \\
\hline 2005 & 98 & $11: 38: 17.47$ & -23.14 & 169.26 & 6.10 & 10 & 17 & 342 \\
\hline 2005 & 99 & $15: 16: 27.89$ & 56.17 & -154.52 & 6.00 & 14 & 98 & 17 \\
\hline 2005 & 100 & 10:29:11.28 & -1.64 & 99.61 & 6.70 & 19 & 77 & 278 \\
\hline 2005 & 100 & $11: 14: 19.62$ & -1.71 & 99.78 & 6.50 & 30 & 77 & 278 \\
\hline 2005 & 100 & $17: 24: 39.40$ & -1.59 & 99.72 & 6.40 & 30 & 77 & 278 \\
\hline 2005 & 100 & 22:22:15.71 & 35.60 & 140.40 & 6.10 & 43 & 81 & 332 \\
\hline 2005 & 101 & 06:11:11.82 & 2.17 & 96.76 & 6.10 & 24 & 82 & 279 \\
\hline 2005 & 101 & $12: 20: 05.96$ & -3.48 & 145.91 & 6.70 & 11 & 44 & 316 \\
\hline 2005 & 101 & 14:54:07.03 & -7.29 & -77.89 & 6.10 & 129 & 99 & 107 \\
\hline 2005 & 101 & 17:08:53.94 & -21.98 & 170.61 & 6.80 & 68 & 17 & 347 \\
\hline 2005 & 106 & 16:38:03.90 & 1.81 & 97.66 & 6.40 & 31 & 81 & 280 \\
\hline 2005 & 118 & 14:07:33.70 & 2.13 & 96.80 & 6.30 & 22 & 82 & 279 \\
\hline 2005 & 130 & 01:09:05.10 & -6.23 & 103.14 & 6.40 & 17 & 72 & 277 \\
\hline 2005 & 132 & $11: 15: 35.34$ & -57.38 & -139.23 & 6.50 & 10 & 35 & 137 \\
\hline 2005 & 134 & 05:05:18.48 & 0.59 & 98.46 & 6.80 & 34 & 80 & 279 \\
\hline 2005 & 138 & 09:10:53.65 & -56.41 & -26.86 & 6.00 & 102 & 83 & 168 \\
\hline 2005 & 138 & 10:27:06.41 & -15.32 & -173.24 & 6.20 & 10 & 26 & 27 \\
\hline 2005 & 138 & $11: 37: 41.74$ & 5.44 & 93.36 & 6.20 & 2 & 87 & 280 \\
\hline 2005 & 139 & 01:54:52.85 & 1.99 & 97.04 & 6.90 & 30 & 82 & 279 \\
\hline 2005 & 140 & $12: 40: 42.41$ & -24.53 & 178.84 & 6.00 & 565 & 15 & 15 \\
\hline 2005 & 155 & $14: 50: 48.85$ & -6.34 & 146.81 & 6.10 & 43 & 41 & 315 \\
\hline 2005 & 159 & 06:28:10.92 & 2.17 & 96.72 & 6.10 & 23 & 82 & 279 \\
\hline 2005 & 163 & $19: 26: 24.80$ & -56.29 & -27.08 & 6.00 & 94 & 83 & 168 \\
\hline 2005 & 164 & 07:02:33.09 & 2.09 & 126.57 & 6.00 & 10 & 60 & 301 \\
\hline 2005 & 164 & 22:44:33.90 & -19.99 & -69.20 & 7.80 & 115 & 96 & 122 \\
\hline 2005 & 165 & $17: 10: 16.64$ & 51.23 & 179.41 & 6.80 & 51 & 90 & 3 \\
\hline 2005 & 166 & 10:13:59.39 & -4.59 & 153.19 & 6.30 & 74 & 40 & 325 \\
\hline
\end{tabular}


Table B.2 - continued

\begin{tabular}{|c|c|c|c|c|c|c|c|c|}
\hline Year & Day & Time & Lat & Long & Magnitude & Depth & Dist & BAZ \\
\hline 2005 & 166 & 19:52:24.82 & -44.87 & -80.56 & 6.50 & 10 & 72 & 134 \\
\hline 2005 & 177 & 08:23:03.87 & 1.77 & 125.82 & 6.00 & 91 & 61 & 300 \\
\hline 2005 & 186 & 01:52:02.95 & 1.82 & 97.08 & 6.80 & 21 & 82 & 279 \\
\hline 2005 & 191 & 04:46:31.24 & -36.31 & -97.26 & 6.10 & 10 & 67 & 119 \\
\hline 2005 & 192 & 12:01:35.23 & -23.82 & -111.68 & 6.00 & 10 & 63 & 100 \\
\hline 2005 & 204 & $07: 34: 56.77$ & 35.50 & 139.98 & 6.10 & 61 & 81 & 332 \\
\hline 2005 & 204 & 08:51:43.57 & -30.25 & -178.07 & 6.00 & 10 & 11 & 36 \\
\hline 2005 & 205 & 15:42:06.21 & 7.92 & 92.19 & 7.50 & 16 & 89 & 281 \\
\hline 2005 & 218 & 09:56:13.54 & -19.61 & -175.85 & 6.00 & 205 & 21 & 25 \\
\hline 2005 & 219 & $11: 35: 26.14$ & -14.45 & -177.29 & 6.00 & 10 & 26 & 18 \\
\hline 2005 & 221 & 05:26:17.53 & -20.94 & 173.82 & 6.10 & 23 & 18 & 357 \\
\hline 2005 & 221 & $14: 12: 21.17$ & -21.10 & 173.87 & 6.10 & 35 & 18 & 357 \\
\hline 2005 & 223 & 09:08:46.81 & -22.68 & 169.51 & 6.20 & 10 & 17 & 343 \\
\hline 2005 & 225 & $07: 36: 52.77$ & 20.13 & 145.80 & 6.00 & 48 & 65 & 330 \\
\hline 2005 & 228 & 02:46:28.40 & 38.28 & 142.04 & 7.20 & 36 & 83 & 335 \\
\hline 2005 & 236 & 10:15:28.19 & 38.56 & 142.99 & 6.20 & 10 & 83 & 335 \\
\hline 2005 & 242 & 18:10:45.46 & 38.48 & 143.18 & 6.20 & 21 & 82 & 336 \\
\hline 2005 & 248 & 07:37:31.31 & -56.41 & -142.39 & 6.20 & 10 & 33 & 136 \\
\hline 2005 & 249 & 01:16:02.35 & 24.08 & 122.19 & 6.10 & 32 & 80 & 312 \\
\hline 2005 & 252 & 07:26:43.73 & -4.54 & 153.47 & 7.70 & 90 & 39 & 325 \\
\hline 2005 & 268 & $12: 55: 46.60$ & -17.51 & 167.80 & 6.20 & 30 & 22 & 342 \\
\hline 2005 & 269 & 01:55:37.67 & -5.68 & -76.40 & 7.50 & 115 & 101 & 106 \\
\hline 2005 & 272 & $15: 50: 24.03$ & -5.44 & 151.84 & 6.70 & 25 & 39 & 322 \\
\hline 2005 & 272 & $18: 23: 25.98$ & -5.56 & 151.87 & 6.20 & 28 & 39 & 322 \\
\hline 2005 & 278 & 10:07:22.37 & -20.53 & -174.28 & 6.00 & 15 & 21 & 30 \\
\hline 2005 & 284 & 15:05:39.66 & 4.82 & 95.10 & 6.00 & 30 & 85 & 280 \\
\hline 2005 & 288 & 10:06:17.01 & 46.82 & 154.11 & 6.10 & 42 & 88 & 346 \\
\hline 2005 & 288 & 15:51:07.21 & 25.32 & 123.36 & 6.50 & 183 & 80 & 314 \\
\hline 2005 & 292 & 11:44:42.79 & 36.40 & 140.84 & 6.50 & 32 & 81 & 333 \\
\hline 2005 & 296 & 10:08:14.74 & 37.38 & 134.56 & 6.00 & 380 & 85 & 329 \\
\hline 2005 & 302 & 04:05:56.04 & -45.21 & 96.90 & 6.50 & 8 & 56 & 237 \\
\hline 2005 & 309 & 10:48:21.28 & -3.16 & 148.14 & 6.40 & 25 & 43 & 319 \\
\hline 2005 & 318 & 21:38:51.42 & 38.11 & 144.90 & 7.00 & 11 & 82 & 337 \\
\hline 2005 & 321 & $19: 26: 56.43$ & -22.32 & -67.89 & 6.90 & 162 & 9518 & 124 \\
\hline 2005 & 323 & 14:10:13.03 & 2.16 & 96.79 & 6.50 & 21 & 82 & 279 \\
\hline 2005 & 324 & 12:53:02.95 & 53.84 & -164.09 & 6.20 & 30 & 94 & 12 \\
\hline 2005 & 325 & 15:36:30.98 & 31.02 & 130.00 & 6.20 & 145 & 81 & 322 \\
\hline 2005 & 326 & $15: 11: 31.58$ & -5.15 & 145.28 & 6.20 & 68 & 43 & 314 \\
\hline 2005 & 332 & $16: 41: 32.54$ & 20.29 & 146.04 & 6.10 & 41 & 65 & 330 \\
\hline 2005 & 334 & $16: 53: 42.47$ & 6.27 & 124.03 & 6.30 & 13 & 65 & 302 \\
\hline 2005 & 336 & 13:13:09.52 & 38.09 & 142.12 & 6.50 & 29 & 82 & 335 \\
\hline 2005 & 337 & $16: 10: 42.40$ & 29.34 & 130.26 & 6.00 & 47 & 80 & 322 \\
\hline 2005 & 341 & $23: 32: 51.55$ & -30.01 & -177.64 & 6.40 & 21 & 11 & 37 \\
\hline 2005 & 342 & 09:01:27.11 & -5.41 & 146.95 & 6.10 & 216 & 42 & 316 \\
\hline 2005 & 345 & 14:20:43.79 & -6.57 & 152.20 & 6.60 & 10 & 38 & 322 \\
\hline 2005 & 347 & 03:16:06.38 & -15.27 & -178.57 & 6.80 & 10 & 24 & 16 \\
\hline 2005 & 350 & 18:32:16.42 & 38.51 & 141.90 & 6.00 & 42 & 83 & 335 \\
\hline
\end{tabular}


Table B.2 - continued

\begin{tabular}{|c|c|c|r|r|r|r|r|r|}
\hline Year & Day & Time & Lat & Long & Magnitude & Depth & Dist & BAZ \\
\hline 2005 & 354 & $05: 51: 12.56$ & 12.23 & 140.99 & 6.10 & 22 & 60 & 321 \\
2005 & 355 & $07: 09: 05.17$ & -0.07 & 124.67 & 6.30 & 25 & 60 & 298 \\
2005 & 356 & $12: 20: 02.94$ & -54.72 & -135.87 & 6.30 & 10 & 36 & 132 \\
\hline
\end{tabular}

Table B.3: Events used in receiver functions for the CNIPSE stations. Distance in degrees (Dist) and backazimuth (BAZ) are calculated from station YUPC

\begin{tabular}{|r|c|c|r|r|r|r|r|r|}
\hline Year & Day & Time & Lat & Long & Magnitude & Depth & Dist & BAZ \\
\hline 2001 & 44 & $19: 28: 30.26$ & -4.68 & 102.56 & 7.40 & 36 & 74 & 277 \\
2001 & 45 & $14: 04: 25.61$ & -19.49 & 177.33 & 6.10 & 10 & 19 & 6 \\
2001 & 47 & $05: 59: 09.48$ & -7.16 & 117.49 & 6.10 & 521 & 61 & 285 \\
2001 & 55 & $07: 23: 48.73$ & 1.27 & 126.25 & 7.10 & 35 & 60 & 299 \\
2001 & 55 & $16: 33: 44.68$ & 1.55 & 126.43 & 6.20 & 33 & 60 & 299 \\
2001 & 57 & $05: 58: 22.43$ & 46.81 & 144.52 & 6.10 & 392 & 89 & 339 \\
2001 & 65 & $09: 17: 36.08$ & -54.59 & 157.27 & 6.40 & 10 & 20 & 211 \\
2001 & 78 & $05: 52: 15.89$ & -4.03 & 128.02 & 6.50 & 33 & 55 & 296 \\
2001 & 82 & $11: 30: 10.52$ & 44.07 & 148.05 & 6.00 & 33 & 86 & 341 \\
2001 & 83 & $06: 27: 53.58$ & 34.08 & 132.53 & 6.80 & 50 & 82 & 325 \\
2001 & 94 & $07: 44: 11.20$ & -5.18 & 132.37 & 6.40 & 33 & 51 & 299 \\
2001 & 97 & $23: 17: 37.92$ & -27.55 & -176.34 & 6.20 & 33 & 13 & 35 \\
2001 & 99 & $09: 00: 57.17$ & -32.67 & -73.11 & 6.70 & 11 & 85 & 128 \\
2001 & 104 & $23: 27: 26.66$ & 30.09 & 141.77 & 6.00 & 10 & 75 & 330 \\
2001 & 109 & $03: 13: 25.58$ & -7.45 & 155.89 & 6.00 & 12 & 35 & 325 \\
2001 & 109 & $20: 58: 26.14$ & -7.31 & 155.96 & 6.20 & 20 & 35 & 325 \\
2001 & 109 & $21: 434: 2.28$ & -7.41 & 155.87 & 6.7 & 170 & 35 & 325 \\
2001 & 118 & $04: 49: 53.43$ & -18.06 & -176.94 & 351 & 6.90 & 21 & 20 \\
2001 & 127 & $00: 33: 23.80$ & -56.19 & -144.47 & 6.20 & 10 & 32 & 137 \\
2001 & 129 & $17: 38: 26.12$ & -10.32 & 161.23 & 6.30 & 67 & 31 & 332 \\
2001 & 139 & $17: 36: 25.59$ & -19.90 & -177.51 & 6.00 & 368 & 19 & 21 \\
2001 & 145 & $00: 40: 50.60$ & 44.27 & 148.39 & 6.70 & 33 & 86 & 341 \\
2001 & 145 & $05: 06: 10.68$ & -7.87 & 110.18 & 6.30 & 143 & 66 & 279 \\
2001 & 146 & $10: 57: 26.15$ & -20.29 & -177.84 & 6.40 & 406 & 19 & 20 \\
2001 & 148 & $08: 37: 05.11$ & -6.61 & 132.35 & 6.00 & 33 & 50 & 298 \\
2001 & 149 & $23: 37: 19.49$ & -7.02 & 155.04 & 6.40 & 14 & 36 & 324 \\
2001 & 156 & $09: 00: 05.38$ & -6.88 & 146.39 & 6.40 & 10 & 41 & 313 \\
2001 & 165 & $19: 48: 47.85$ & 51.16 & -179.83 & 6.50 & 18 & 89 & 3 \\
2001 & 166 & $06: 17: 45.33$ & 18.83 & 146.98 & 6.00 & 33 & 63 & 330 \\
2001 & 167 & $02: 13: 38.18$ & -14.89 & -173.34 & 6.00 & 10 & 25 & 26 \\
2001 & 174 & $20: 33: 14.13$ & -16.26 & -73.64 & 8.40 & 33 & 96 & 116 \\
2001 & 177 & $04: 18: 31.38$ & -17.75 & -71.65 & 6.70 & 24 & 96 & 118 \\
2001 & 182 & $01: 46: 06.13$ & -4.31 & 152.96 & 6.10 & 28 & 40 & 323 \\
2001 & 184 & $13: 10: 42.60$ & 21.64 & 142.98 & 6.50 & 290 & 67 & 327 \\
2001 & 185 & $07: 06: 31.60$ & -21.73 & -176.71 & 6.50 & 184 & 18 & 25 \\
\hline
\end{tabular}


Table B.3 - continued

\begin{tabular}{|l|c|c|r|r|r|r|r|r|}
\hline Year & Day & Time & Lat & Long & Magnitude & Depth & Dist & BAZ \\
\hline 2001 & 186 & $13: 53: 48.36$ & -16.09 & -73.99 & 6.60 & 62 & 96 & 115 \\
2001 & 188 & $09: 38: 43.52$ & -17.54 & -72.08 & 7.60 & 33 & 96 & 118 \\
2001 & 189 & $17: 54: 18.76$ & -6.66 & 152.11 & 6.20 & 10 & 38 & 320 \\
2001 & 203 & $18: 15: 09.01$ & -5.15 & 132.34 & 6.00 & 33 & 51 & 299 \\
2001 & 218 & $03: 52: 59.51$ & -55.54 & -123.42 & 6.70 & 10 & 44 & 134 \\
2001 & 225 & $20: 11: 23.40$ & 41.05 & 142.31 & 6.40 & 38 & 85 & 335 \\
2001 & 239 & $01: 16: 47.45$ & 1.09 & 126.36 & 6.10 & 33 & 60 & 299 \\
2001 & 245 & $02: 25: 54.09$ & 0.89 & 82.50 & 6.10 & 10 & 93 & 269 \\
2001 & 245 & $10: 06: 51.50$ & -54.36 & -137.02 & 6.30 & 10 & 36 & 132 \\
2001 & 250 & $02: 45: 59$ & -13.17 & 97.30 & 6.20 & 10 & 73 & 267 \\
2001 & 255 & $08: 48: 37.27$ & -20.99 & -179.11 & 6.50 & 608 & 18 & 17 \\
2001 & 255 & $22: 23: 44.09$ & 27.69 & 141.91 & 6.00 & 33 & 73 & 329 \\
2001 & 258 & $15: 04: 34.08$ & -22.39 & -175.01 & 6.00 & 10 & 18 & 30 \\
2001 & 261 & $02: 19: 30.79$ & -7.51 & 127.74 & 6.00 & 131 & 53 & 293 \\
2001 & 272 & $02: 40: 07.38$ & -18.50 & 168.16 & 6.40 & 33 & 21 & 340 \\
2001 & 273 & $19: 01: 18.71$ & -18.42 & 168.12 & 6.20 & 33 & 21 & 340 \\
2001 & 275 & $00: 48: 18.79$ & -16.18 & -173.82 & 6.20 & 106 & 24 & 26 \\
2001 & 280 & $02: 21: 09.81$ & -3.30 & 142.93 & 6.20 & 10 & 46 & 311 \\
2001 & 285 & $15: 02: 16.84$ & 12.69 & 144.98 & 7.30 & 37 & 58 & 324 \\
2001 & 286 & $16: 27: 00.07$ & 0.84 & 125.98 & 6.00 & 33 & 60 & 298 \\
2001 & 292 & $03: 28: 44.46$ & -4.10 & 123.91 & 7.50 & 33 & 58 & 293 \\
2001 & 299 & $23: 05: 50.95$ & -18.49 & 168.11 & 6.10 & 33 & 21 & 340 \\
2001 & 304 & $09: 10: 20$ & -5.91 & 150.20 & 7.00 & 33 & 40 & 318 \\
2001 & 309 & $23: 07: 11.72$ & -17.29 & -179.25 & 6.30 & 564 & 21 & 14 \\
2001 & 324 & $21: 08: 18.43$ & -6.88 & 128.92 & 6.20 & 33 & 52 & 294 \\
2001 & 326 & $23: 22: 20.44$ & -16.25 & 178.02 & 6.30 & 10 & 22 & 7 \\
2001 & 332 & $14: 32: 32.72$ & 15.57 & -93.11 & 6.40 & 84 & 101 & 79 \\
2001 & 336 & $02: 47: 56.26$ & -12.74 & 166.66 & 6.00 & 100 & 27 & 341 \\
2001 & 336 & $13: 01: 53.67$ & 39.40 & 141.09 & 6.50 & 123 & 83 & 334 \\
2001 & 337 & $11: 32: 29.80$ & -16.50 & -177.54 & 6.40 & 10 & 23 & 18 \\
2001 & 343 & $18: 15: 02.60$ & 0.00 & 122.87 & 6.10 & 156 & 61 & 295 \\
\hline
\end{tabular}




\section{Appendix C}

\section{SEISMIC ATTENUATION}

Table C.1: TRAP mean $1000 / Q_{p}^{\prime}$ and normalisation factors

\begin{tabular}{|c|c|c|r|c|c|}
\hline & Station & $\begin{array}{c}\text { Mean } \\
1000 / Q_{p}^{\prime}\end{array}$ & $\begin{array}{c}\text { Number } \\
\text { of events }\end{array}$ & $\begin{array}{c}\text { Reference } \\
\text { station } \\
1000 / Q_{p}^{\prime}\end{array}$ & $\begin{array}{c}\text { Normalisation } \\
\text { factor }\end{array}$ \\
\hline \multirow{3}{*}{$\begin{array}{c}\text { Events south of } \\
\text { change in } \\
\text { crustal thickness }\end{array}$} & KAI & 0.73 & 43 & 0.7 & 1.34 \\
& RRU & 0.62 & 43 & 0.7 & 1.34 \\
& PAP & 0.89 & 47 & 0.89 & 1.00 \\
& SUN & 0.60 & 47 & 0.89 & 1.00 \\
& WRR & 0.58 & 47 & 0.89 & 1.00 \\
Events north & TUN & 1.80 & 18 & 0.89 & 1.00 \\
of change in & KAI & 2.03 & 135 & 1.13 & 1.00 \\
crustal thickness & RAE & 1.68 & 135 & 1.13 & 1.00 \\
& PAP & 0.84 & 135 & 1.13 & 1.00 \\
& SUN & 0.83 & 56 & 1.20 & 0.94 \\
& WRR & 0.50 & 56 & 1.20 & 0.94 \\
\hline
\end{tabular}

The relationship between $Q$ and temperature can be estimated using the Andradepseudoperiod model given by Jackson et al. [2004] (equations C.0.1a-C.0.1h), which includes both grain size and frequency dependence. For temperatures close to melting a melt related Gaussian dissipation peak, $Q_{M P}^{-1}$, is superimposed on the background 
dissipation, $Q_{B}^{-1}$.

$$
\begin{aligned}
Q^{-1} & =Q_{B}^{-1}+Q_{M P}^{-1} \\
Q_{B}^{-1} & =\frac{\beta \Gamma(1+n) \omega^{-n} \sin (n \pi / 2)+1 / \eta \omega}{J_{U}+\beta \Gamma(1+n) \omega^{-1} \cos (n \pi / 2)} \\
\omega & =2 \pi / X_{B} \\
X_{B} & =T_{o}\left(d / d_{r}\right)^{-m} \exp \left[\left(-E_{B} / R\right)\left(1 / T-1 / T_{r}\right)\right] \\
Q_{M P}^{-1} & =B \exp \left(-z^{2} / 2\right) \\
X_{P} & =T_{o}\left(d / d_{r}\right)^{-m} \exp \left[\left(-E_{P} / R\right)\left(1 / T-1 / T_{r}\right)\right] \\
B & =B_{0} \varphi^{l} \\
z & =\left(\ln X_{P}-\mu\right) / \sigma
\end{aligned}
$$

$T$ is the temperature $(\mathrm{K}), d$ is the grain size, $\varphi$ is the melt fraction, and $T_{o}$ is the oscillation period. $R$ is the gas constant and $\Gamma$ is the gamma function. The remaining values are constants for the dissipation background (table C.2) and the dissipation peak (table C.3). Extrapolation to different pressures can be achieved using the factor $\exp (-P V / R T)$ where $P$ is the pressure, and $V$ is the activation volume $\left(V=6 \times 10^{-6}\right.$ $\mathrm{m}^{3} / \mathrm{mol}$ ) [Faul et al. 2004].

Table C.2: Constants for dissipation background, $Q_{B}^{-1}$

\begin{tabular}{|c|ccccccc|}
\hline & $\begin{array}{c}d_{r} \\
\mu \mathrm{m}\end{array}$ & $\begin{array}{c}T_{r} \\
\mathrm{~K}\end{array}$ & $\begin{array}{c}E_{B} \\
\mathrm{~kJ} / \mathrm{mol}\end{array}$ & $\begin{array}{c}J_{U}, 10^{-1} \\
\mathrm{GPa}^{-1}\end{array}$ & $n$ & $\begin{array}{c}\beta, 10^{-2} \\
\mathrm{~s}^{-n} \mathrm{GPa}^{-1}\end{array}$ & $\begin{array}{c}\eta, 10^{6} \\
\mathrm{GPa} \mathrm{s}\end{array}$ \\
\hline $\begin{array}{c}\text { melt } \\
\text { free }\end{array}$ & 8.6 & 1373 & 400 & 0.2 & 0.283 & 0.195 & 0.193 \\
\hline $\begin{array}{c}\text { melt } \\
\text { bearing }\end{array}$ & 8.6 & 1373 & 520 & 0.2 & 0.283 & 0.266 & 0.277 \\
\hline
\end{tabular}

Table C.3: Constants for dissipation peak, $Q_{M P}^{-1}$

\begin{tabular}{|c|ccccccc|}
\hline & $d_{r}$ & $T_{r}$ & $E_{P}$ & & & & \\
& $\mu \mathrm{m}$ & $\mathrm{K}$ & $\mathrm{kJ} / \mathrm{mol}$ & $B_{0}$ & $l$ & $\mu$ & $\sigma$ \\
\hline $\begin{array}{c}\text { melt } \\
\text { bearing }\end{array}$ & 8.6 & 1373 & 720 & 0.77 & 0.50 & 2.73 & 2.89 \\
\hline
\end{tabular}




\section{Appendix D}

\section{MAGNETOTELLURICS}

Table D.1: Minimum and maximum skin depths

\begin{tabular}{|c|c|c|}
\hline Station number & $\begin{array}{c}\text { Minimum } \delta \\
(\mathrm{m})\end{array}$ & $\begin{array}{c}\text { Maximum } \delta(\mathrm{km}) \\
\text { TM }\left(\rho_{x y}\right)\end{array}$ \\
\hline 001 & 160 & 210 \\
002 & 360 & 470 \\
003 & 450 & 260 \\
004 & 480 & 600 \\
005 & 160 & 300 \\
006 & 460 & 220 \\
\hline
\end{tabular}




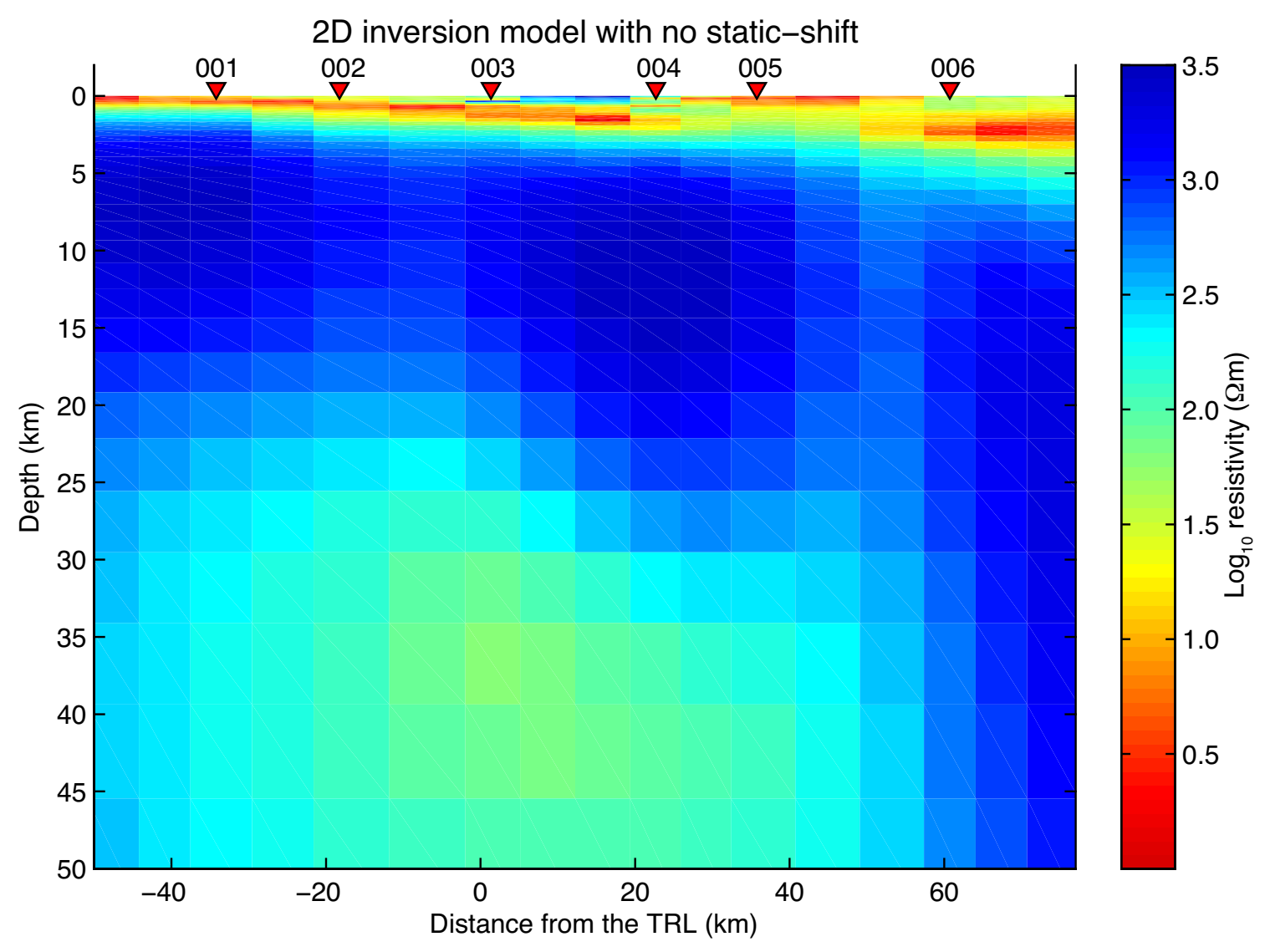

Figure D.1: Inversion model 1. 2D resistivity model from inversion using calculated standard errors and not allowing for static-shift. The forward model for this section has a rms misfit of 4.8 and the model roughness parameter is 90 . 

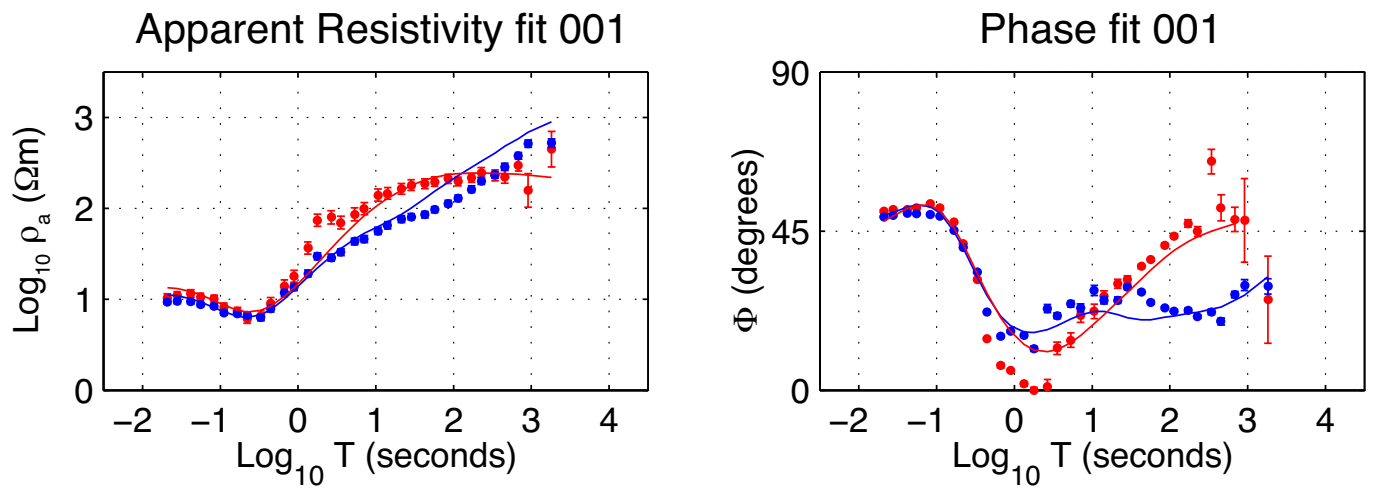

Apparent Resistivity fit 002

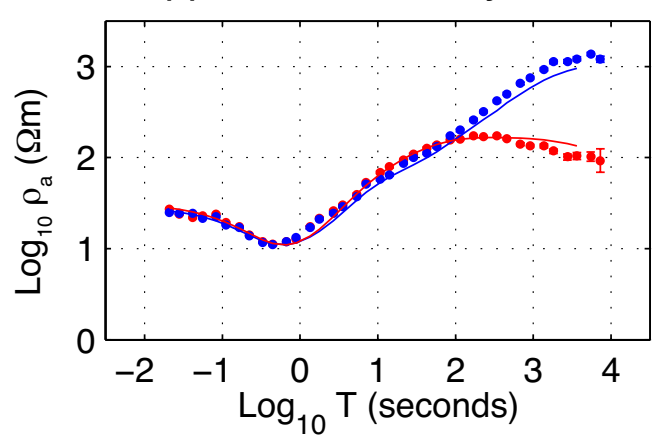

Phase fit 002

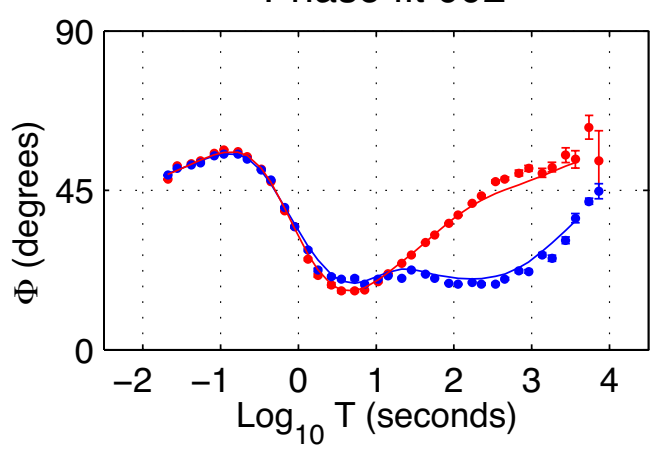

Apparent Resistivity fit 003
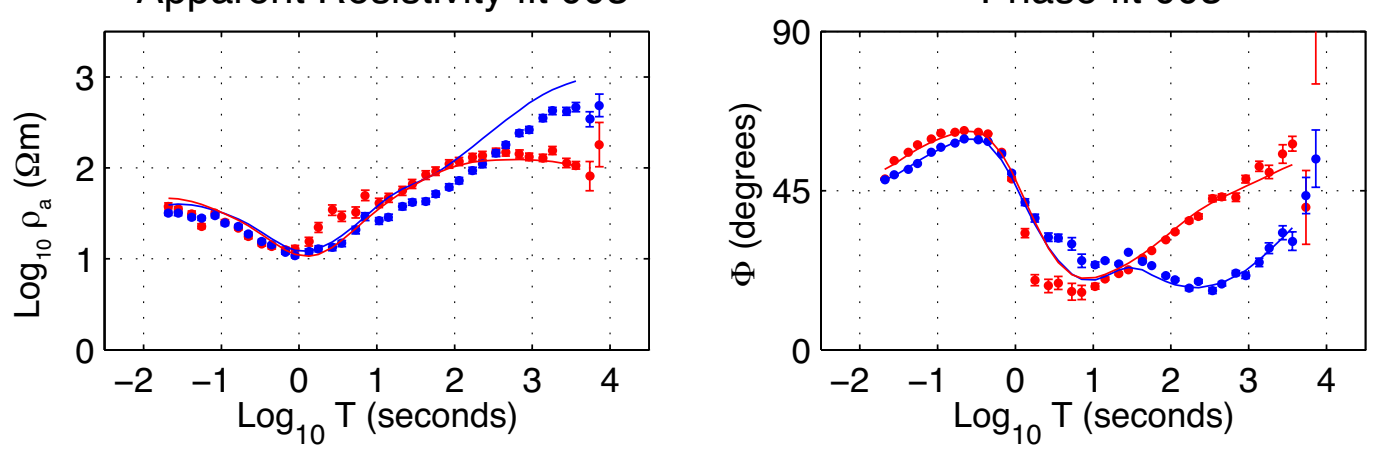

Figure D.2: Fit to inversion 1 model shown in figure D.1 (calculated standard errors and no static-shift) for stations 001-003. The forward model for the TM mode is shown as a red line and the forward model for the TE mode is shown as a blue line. Note the poor fit to the TE mode resistivity data at station 001 and 003. 

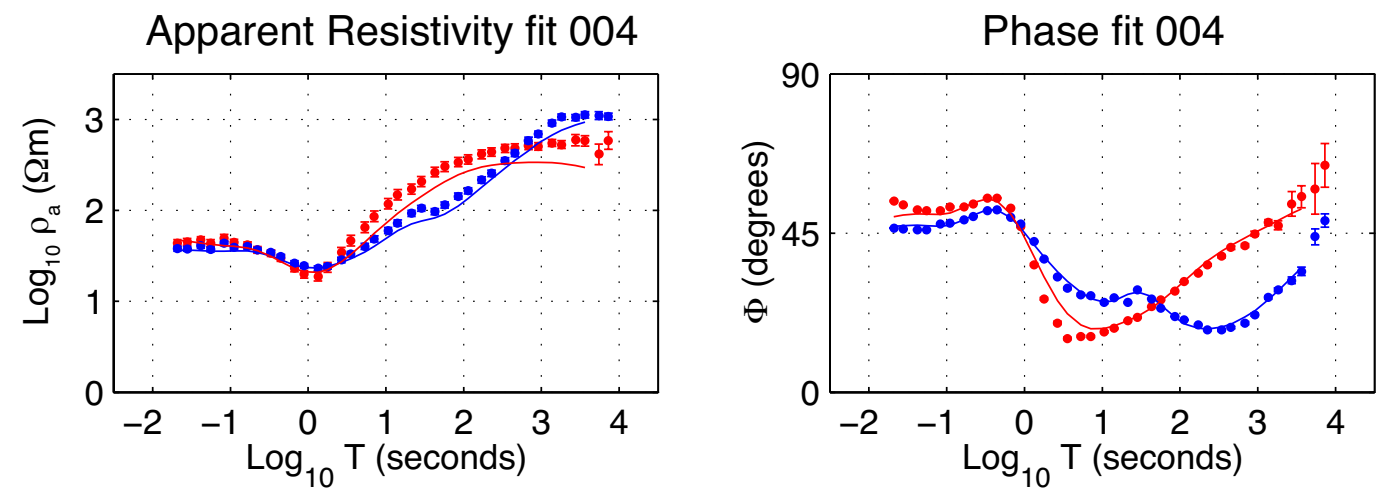

Apparent Resistivity fit 005
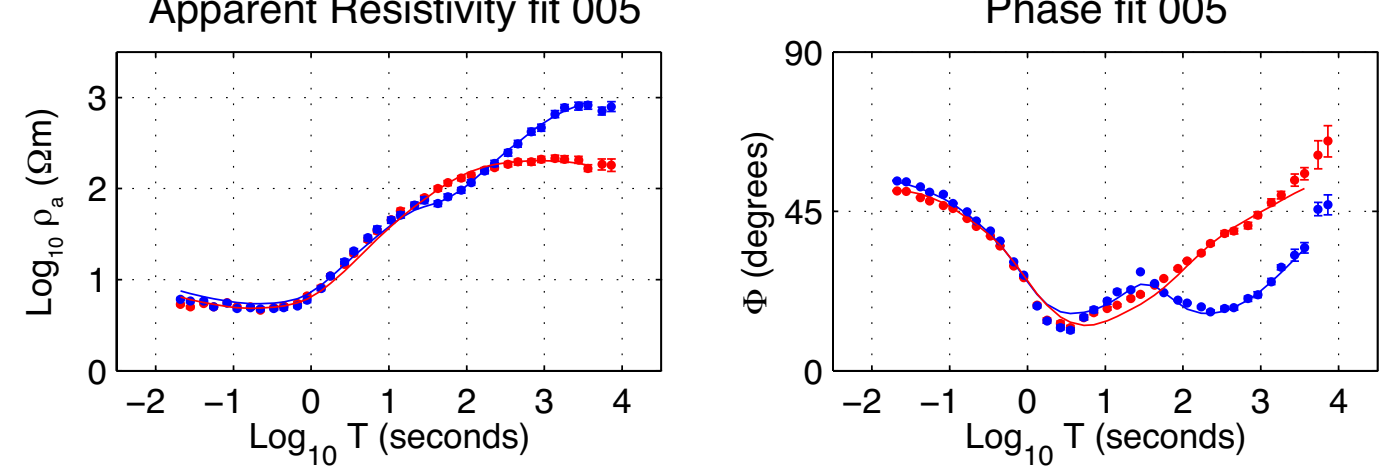

Apparent Resistivity fit 006
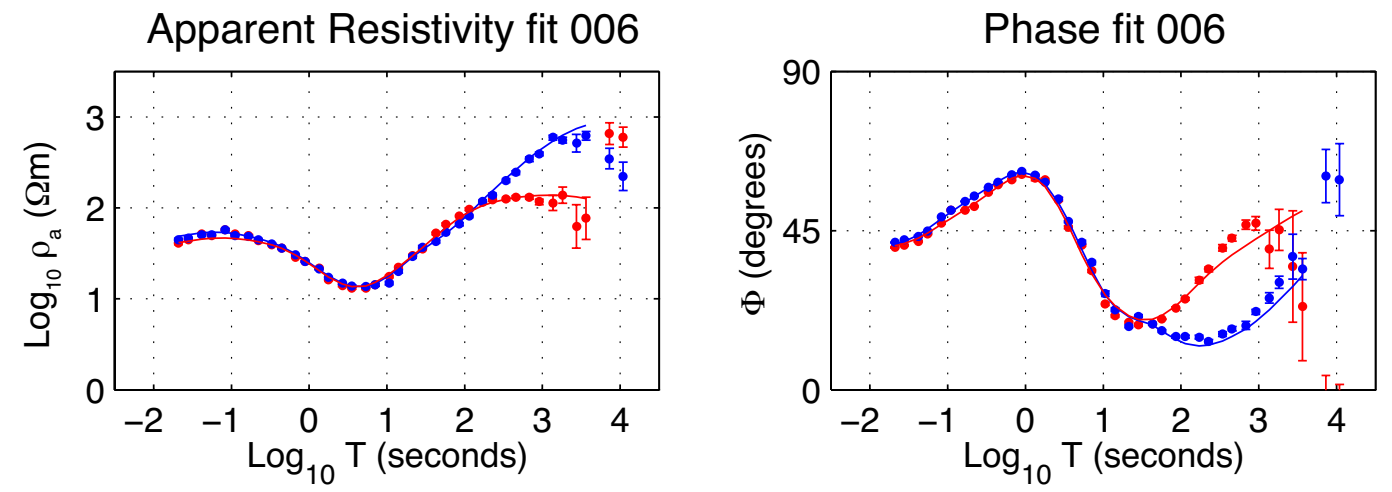

Figure D.3: Fit to inversion model 1 shown in figure D.1 (calculated standard errors and no static-shift) for stations 004-006. The forward model for the TM mode is shown as a red line and the forward model for the TE mode is shown as a blue line. Note the poor fit to the TM mode resistivity data at station 004 . 


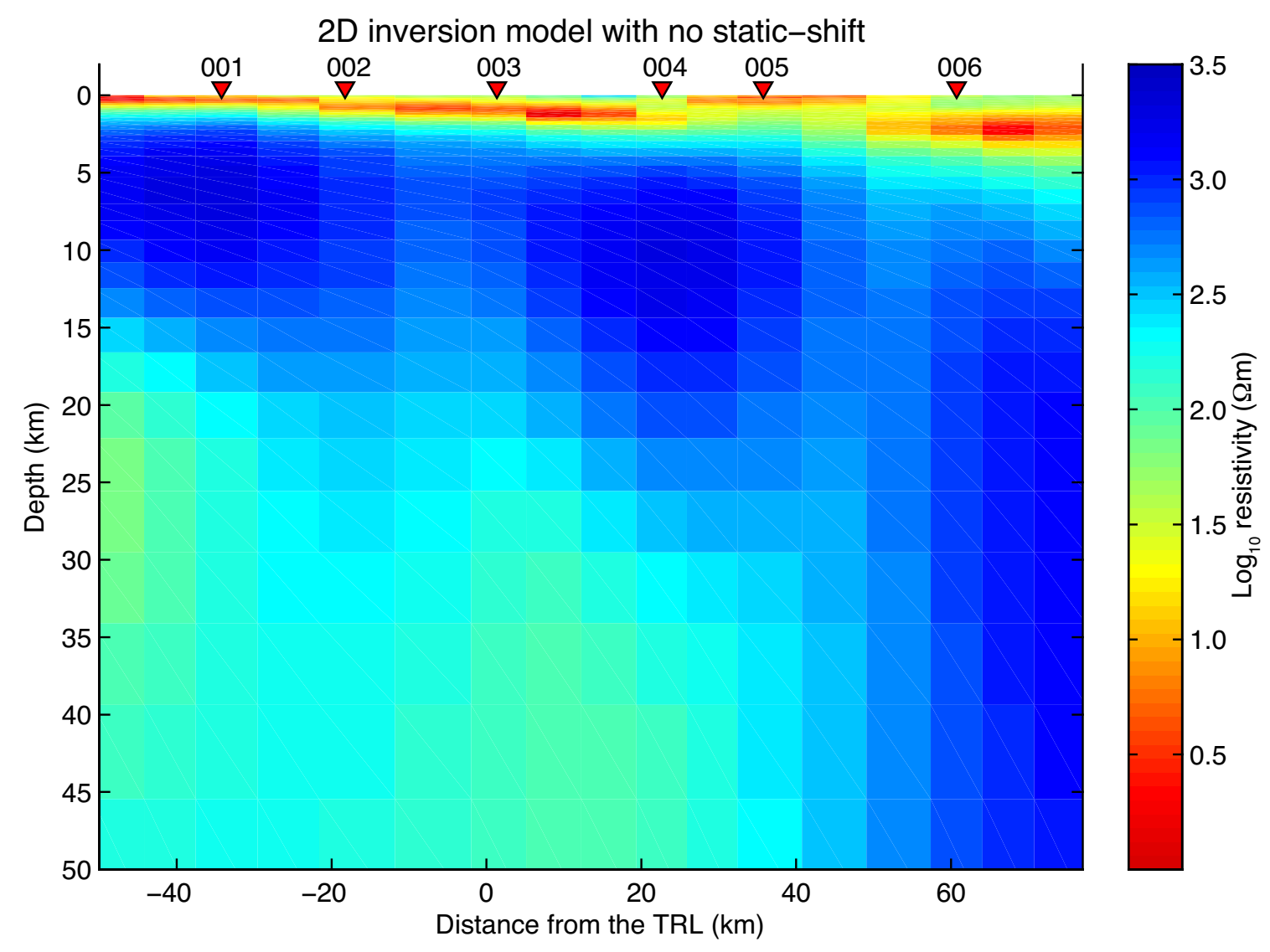

Figure D.4: Inversion model 2. 2D resistivity model from inversion using $5 \%$ minimum errors and not allowing for static-shift. The forward model for this section has a rms misfit of 2.0 and the model roughness parameter is 56 . 

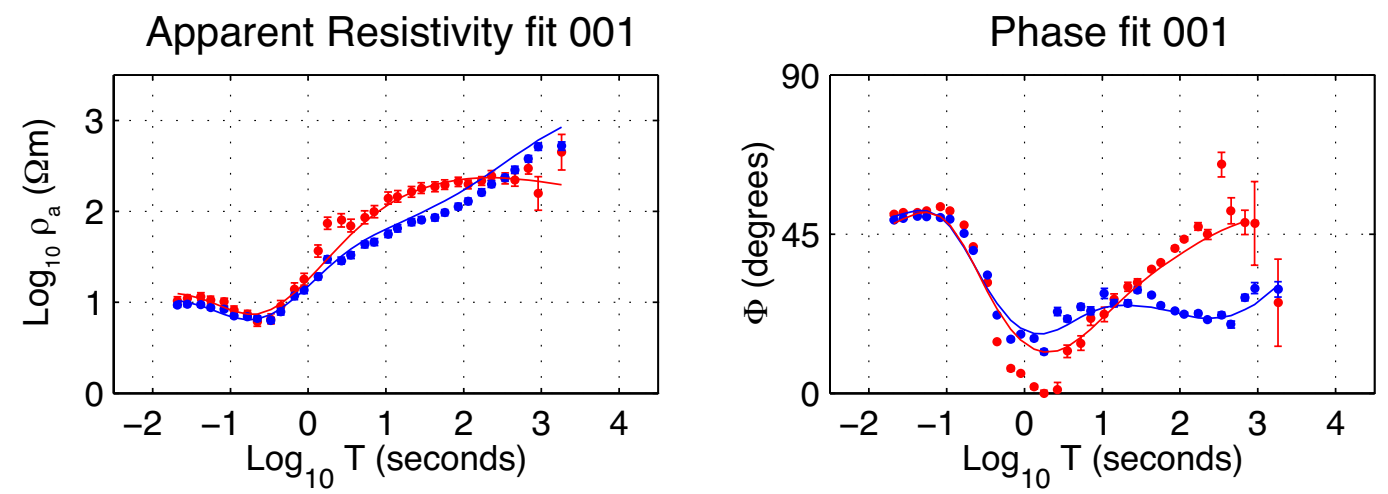

Apparent Resistivity fit 002

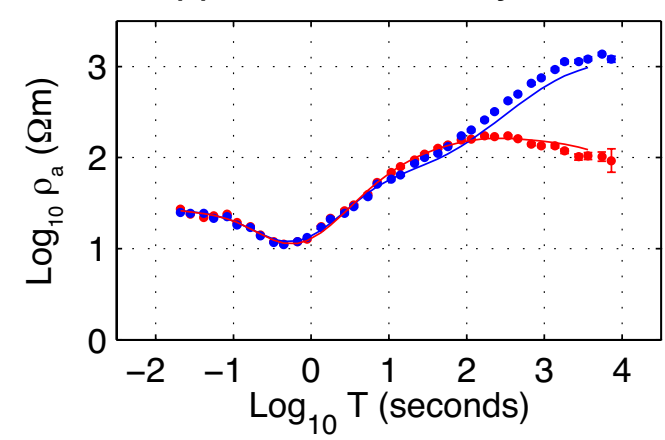

Phase fit 002

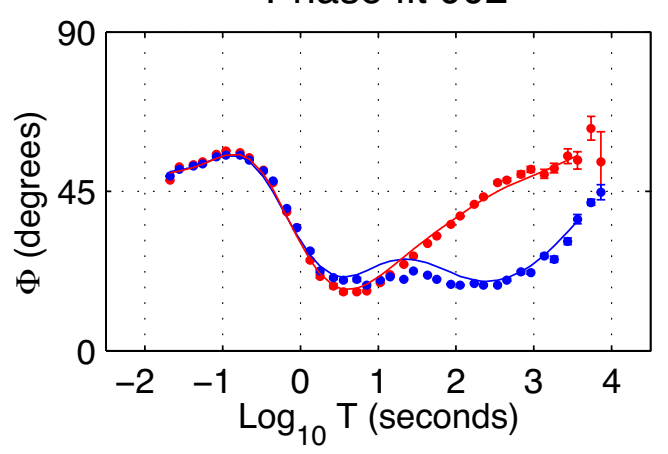

Apparent Resistivity fit 003
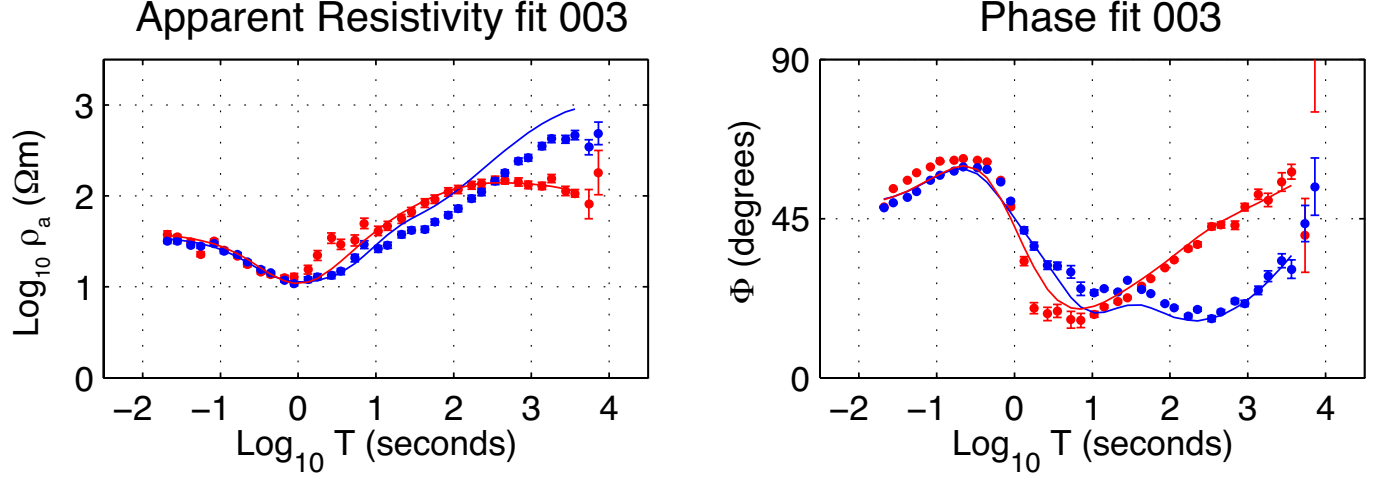

Figure D.5: Fit to inversion model 2 shown in figure D.4 (5\% minimum errors and no static-shift) for stations 001-003. The forward model for the TM mode is shown as a red line and the forward model for the TE mode is shown as a blue line. Note the poor fit to the TE mode resistivity data. 


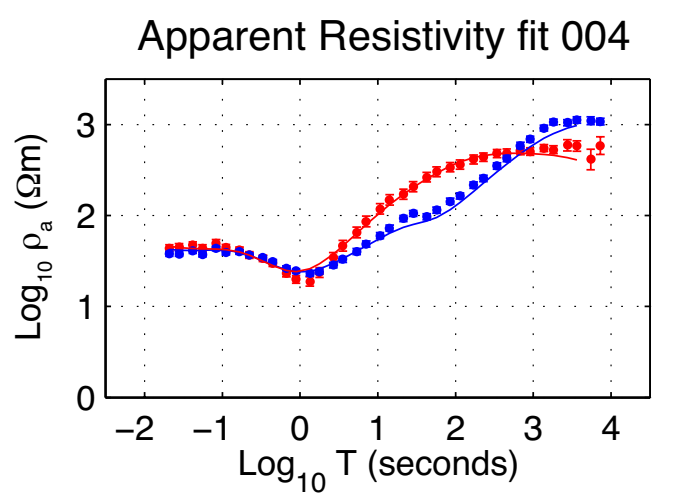

Apparent Resistivity fit 005

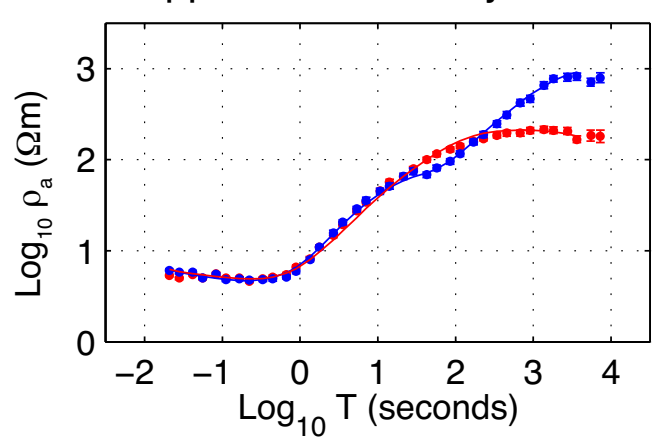

Apparent Resistivity fit 006

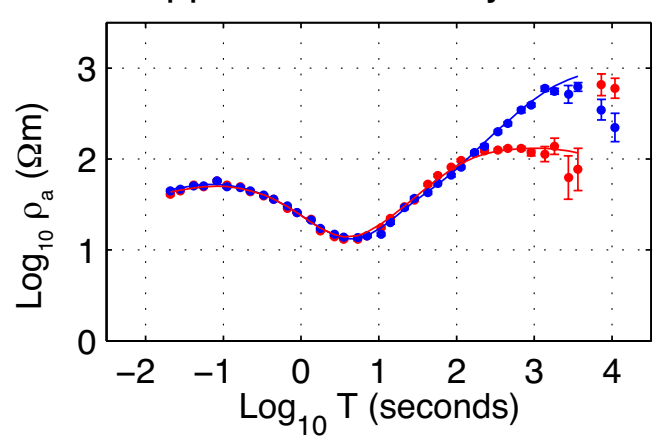

Phase fit 004

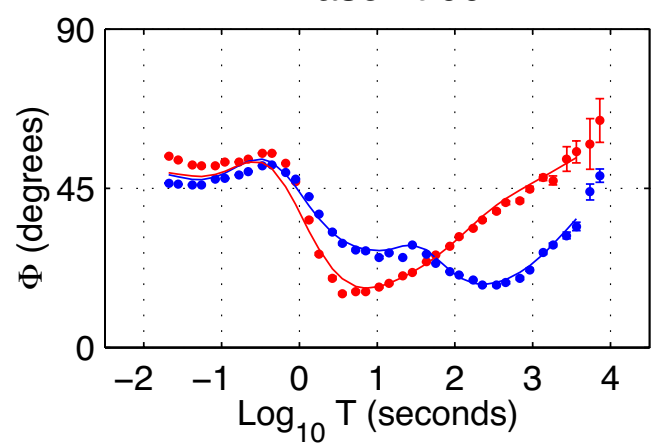

Phase fit 005

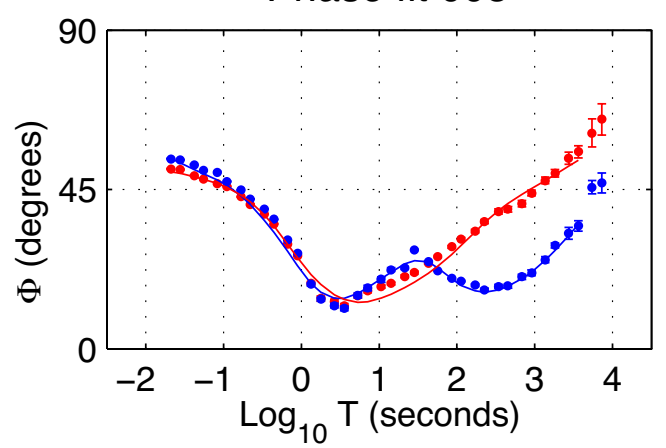

Phase fit 006

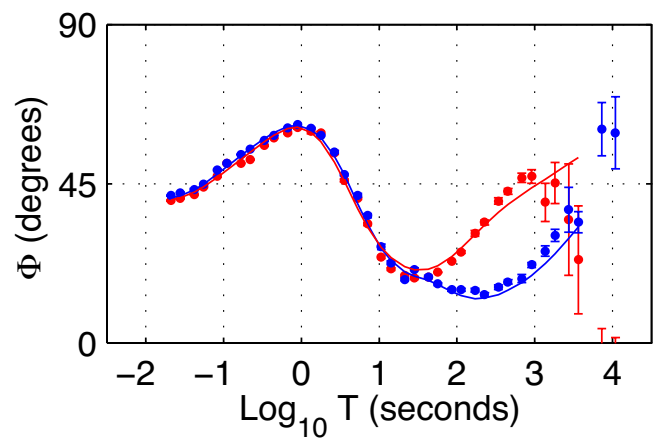

Figure D.6: Fit to inversion model 2 shown in figure D.4 (5\% minimum errors and no static-shift) for stations 004-006. The forward model for the TM mode is shown as a red line and the forward model for the TE mode is shown as a blue line. 


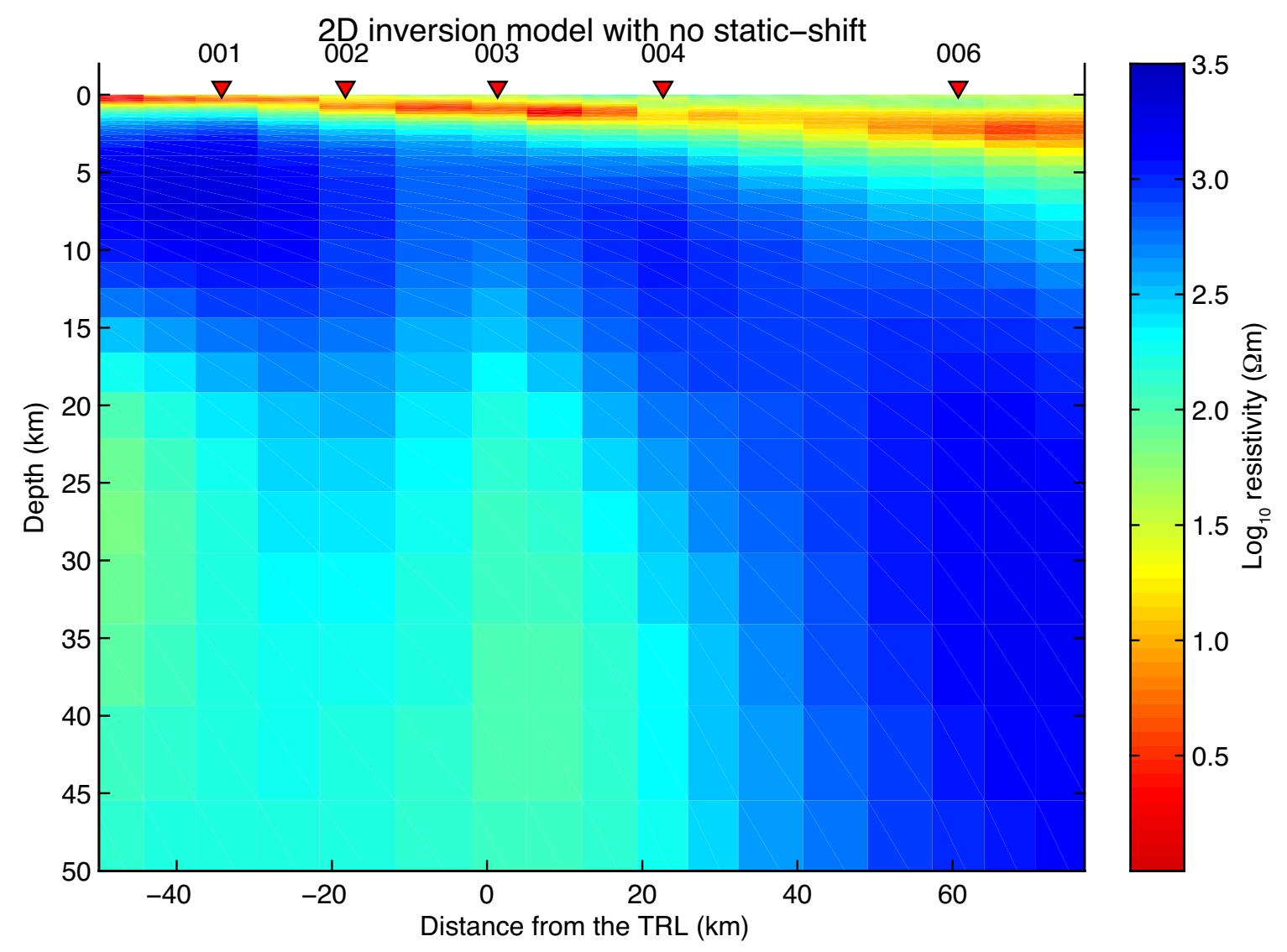

Figure D.7: Inversion model 3. 2D resistivity model from inversion using $5 \%$ minimum errors and not allowing for static-shift. This inversion excluded data from station 005 . The forward model for this section has a rms misfit of 2.1 and the model roughness parameter is 46 . 

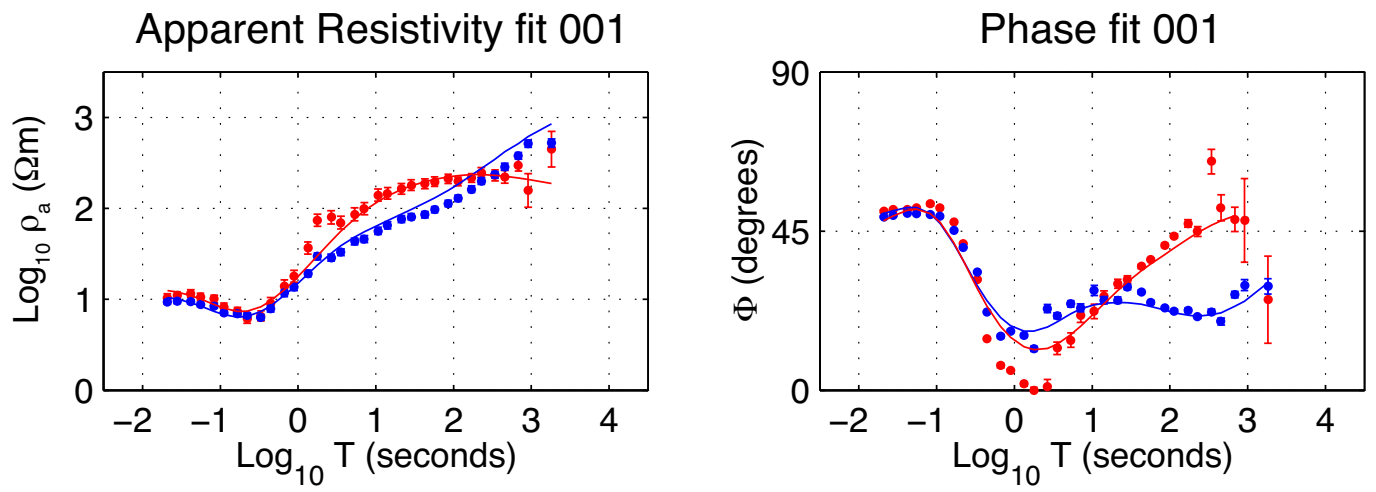

Apparent Resistivity fit 002

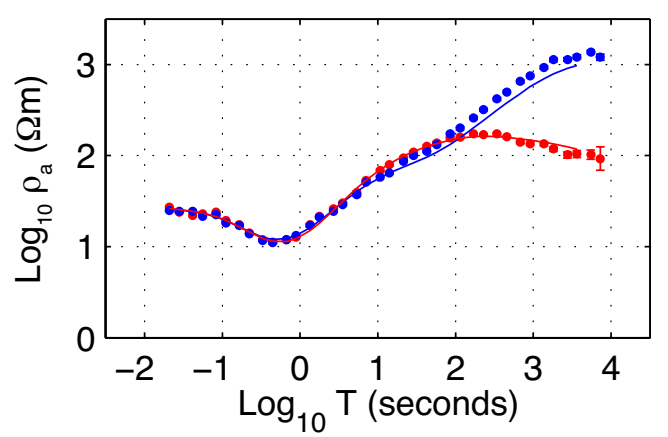

Phase fit 002

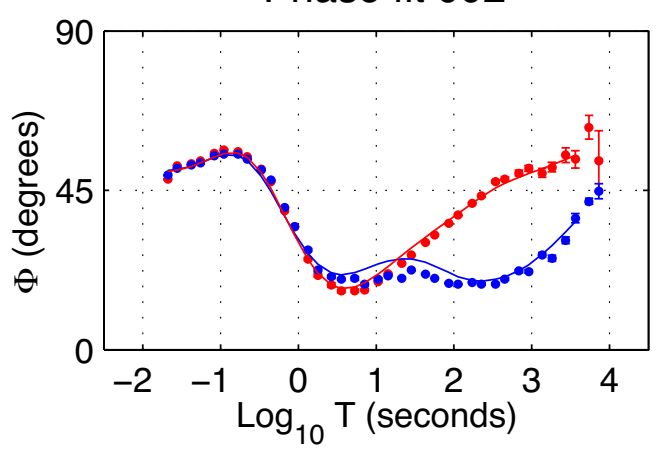

Apparent Resistivity fit 003
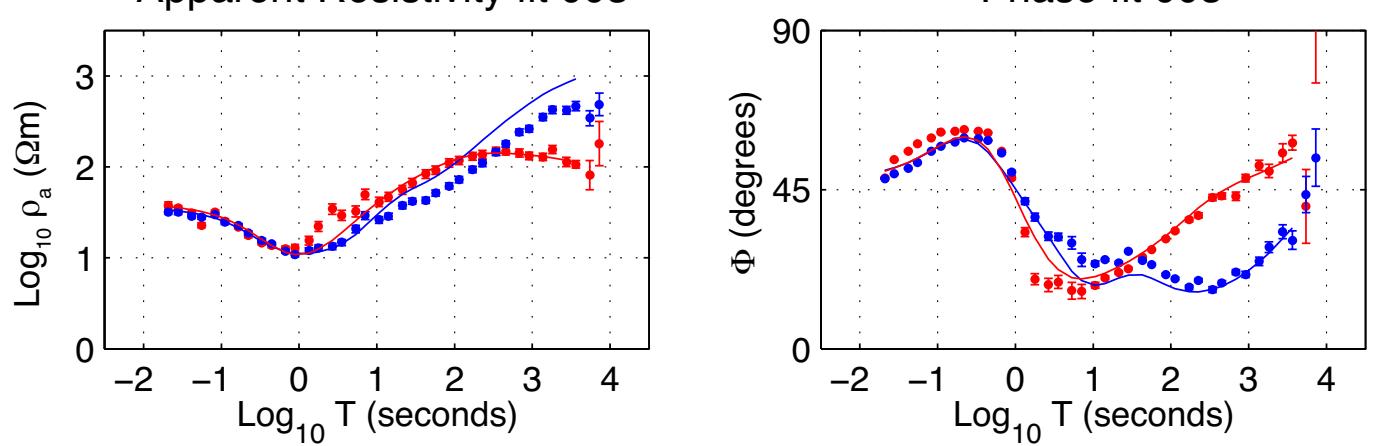

Figure D.8: Fit to inversion model 3 shown in figure D.7 (5\% minimum errors, no static-shift and excluding station 005) for stations 001-003. The forward model for the TM mode is shown as a red line and the forward model for the TE mode is shown as a blue line. Note the poor fit to the TE mode resistivity data. 

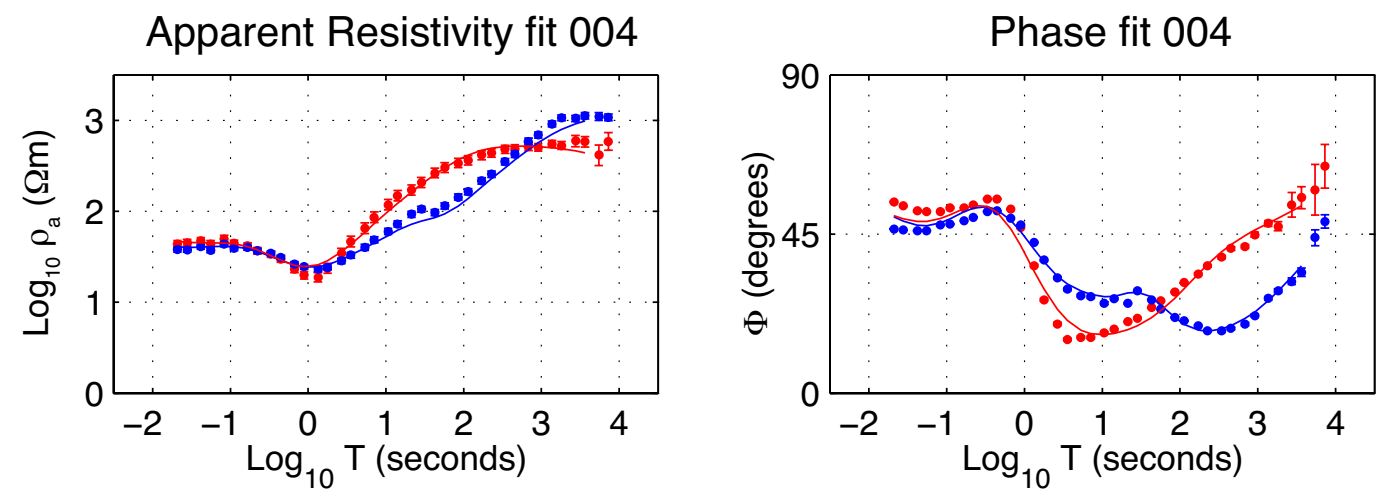

Apparent Resistivity fit 005

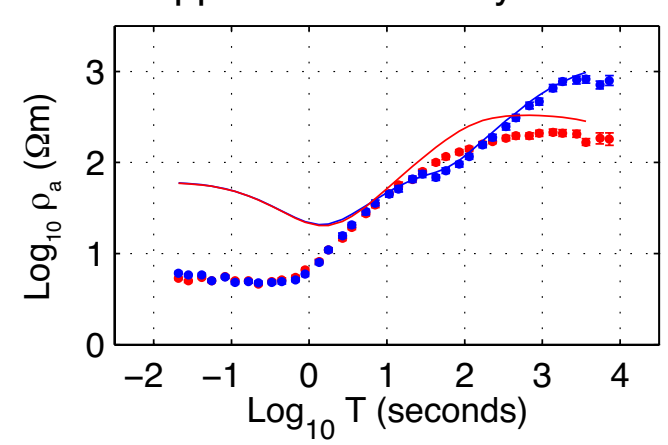

Phase fit 005

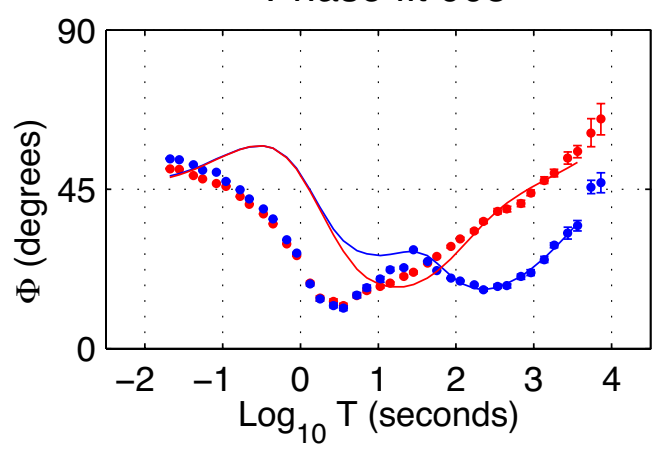

Apparent Resistivity fit 006
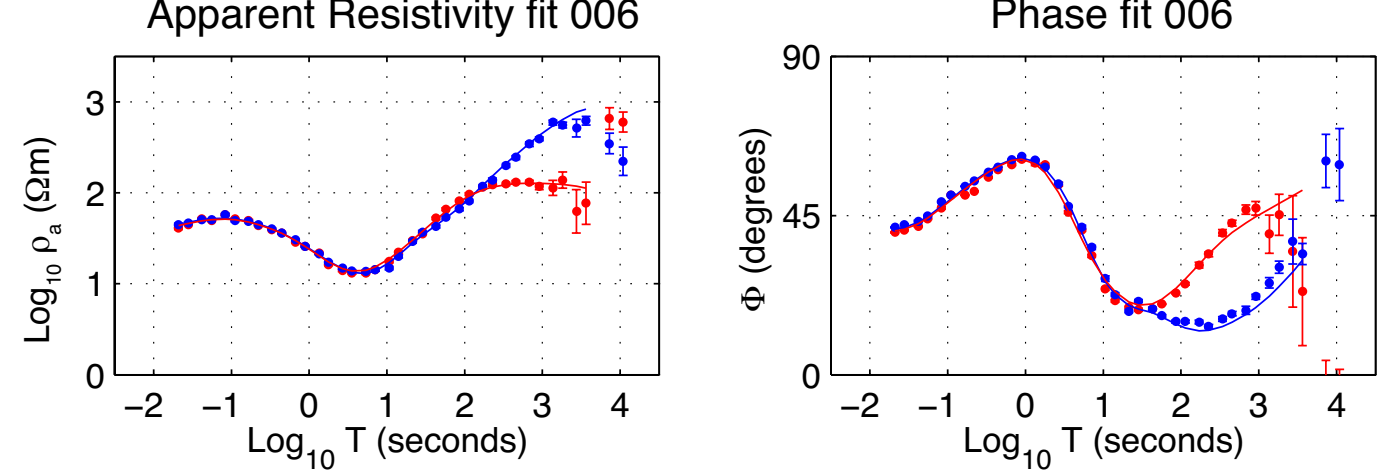

Figure D.9: Fit to inversion model 3 shown in figure D.7 (5\% minimum errors, no static-shift and excluding station 005) for stations 004-006. The forward model for the TM mode is shown as a red line and the forward model for the TE mode is shown as a blue line. Because the data for station 005 was excluded from this inversion this station is poorly fitted. 


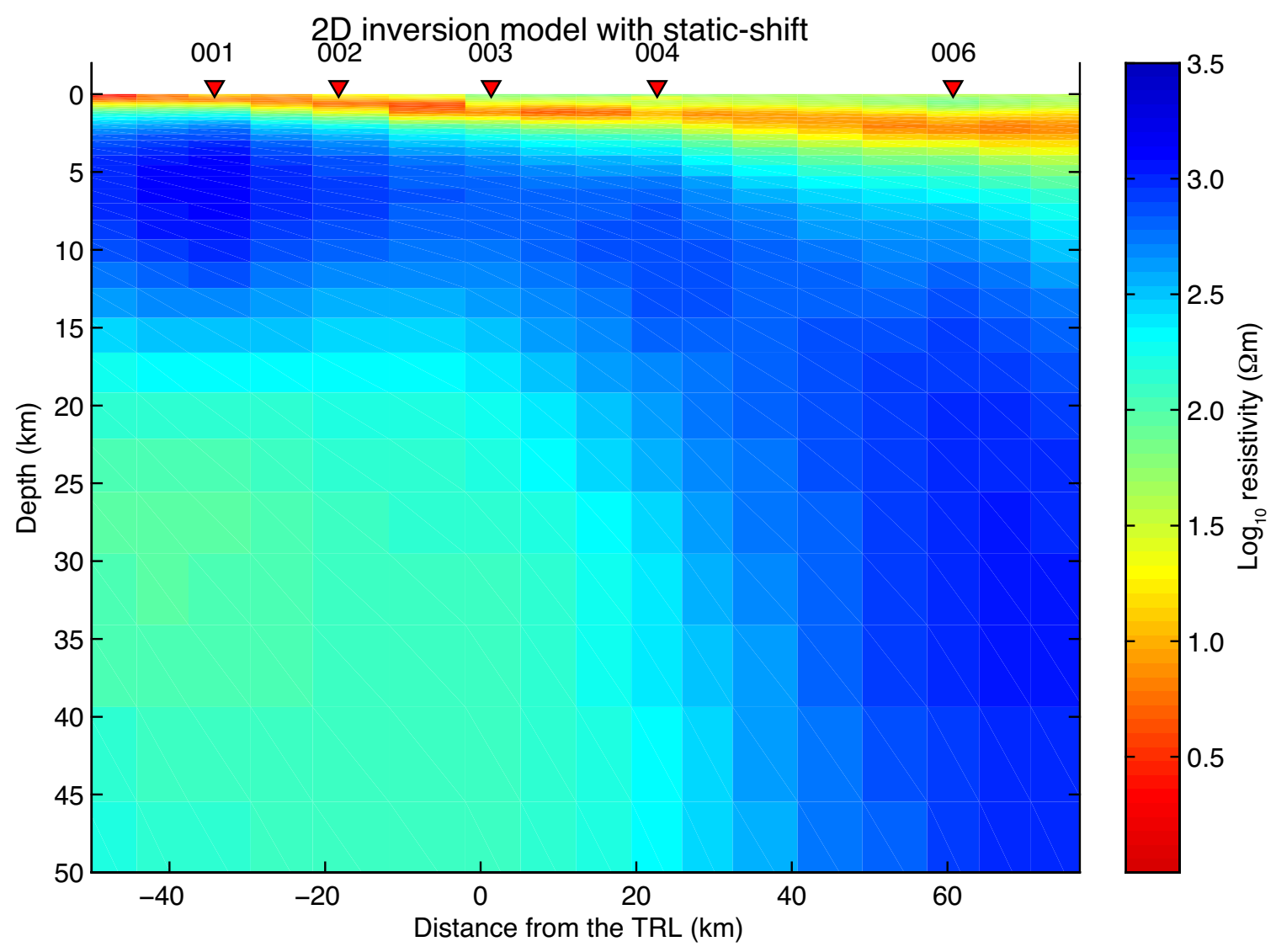

Figure D.10: Inversion model 4. 2D resistivity model from inversion using $5 \%$ minimum errors and including an inversion of static-shift. This inversion excluded data from station 005. The forward model for this section has a rms misfit of 1.5 and the model roughness parameter is 54 . 

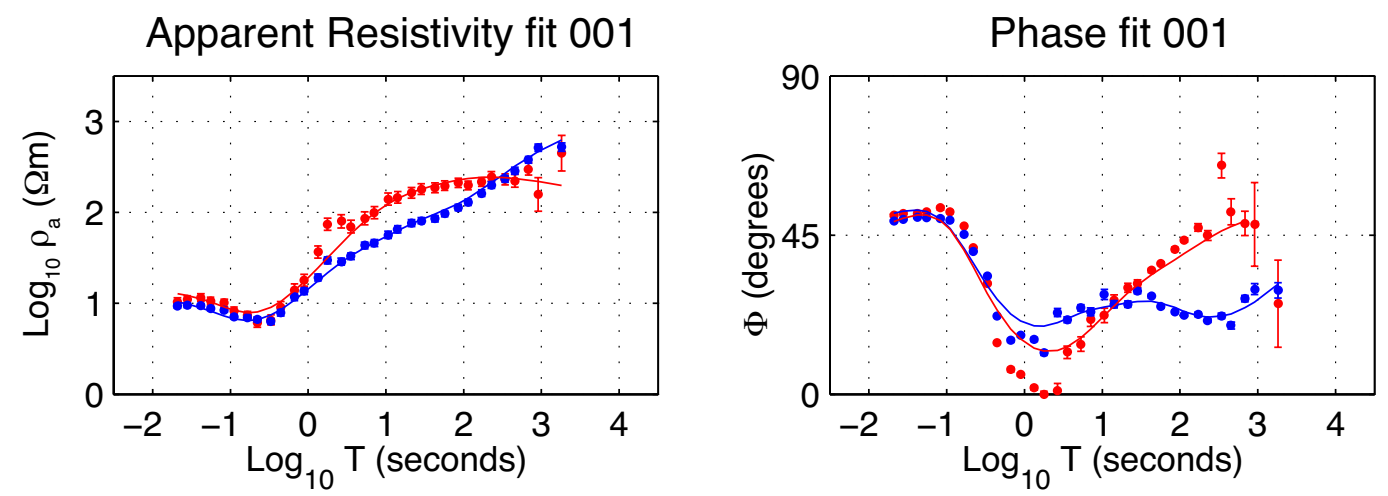

Apparent Resistivity fit 002

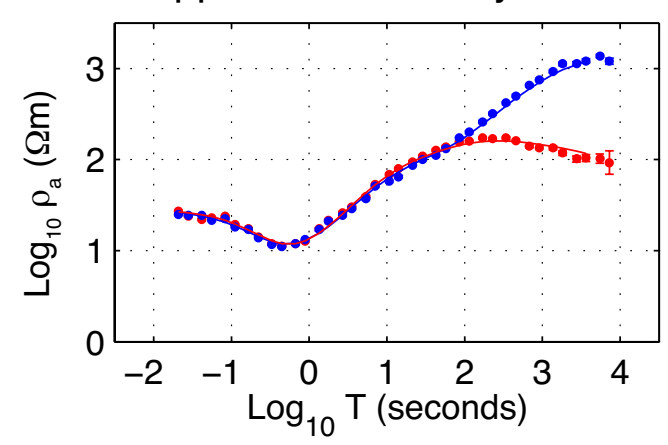

Phase fit 002

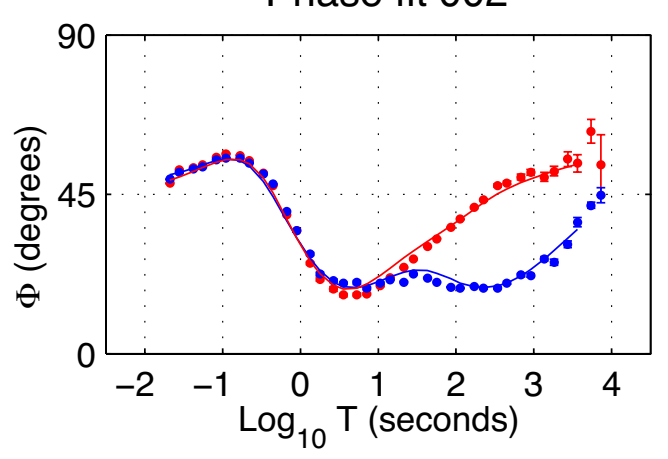

Apparent Resistivity fit 003
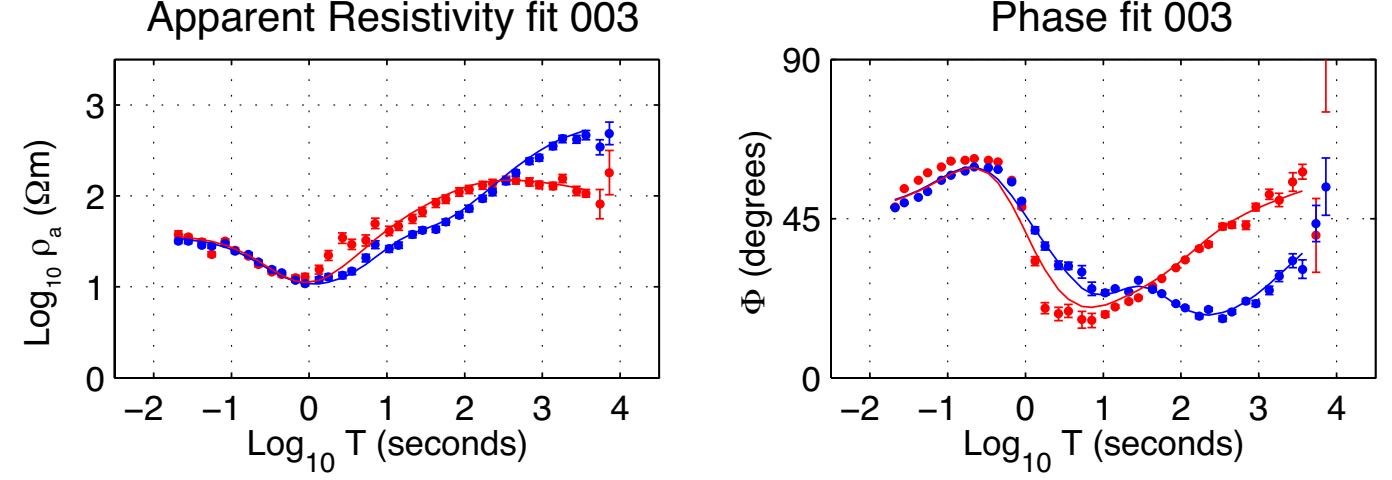

Figure D.11: Fit to inversion model 4 shown in figure D.10 (5\% minimum errors, staticshift and excluding station 005) for stations 001-003. The forward model for the TM mode is shown as a red line and the forward model for the TE mode is shown as a blue line. Inverting for static shift greatly improves the TE mode fit for these stations. 

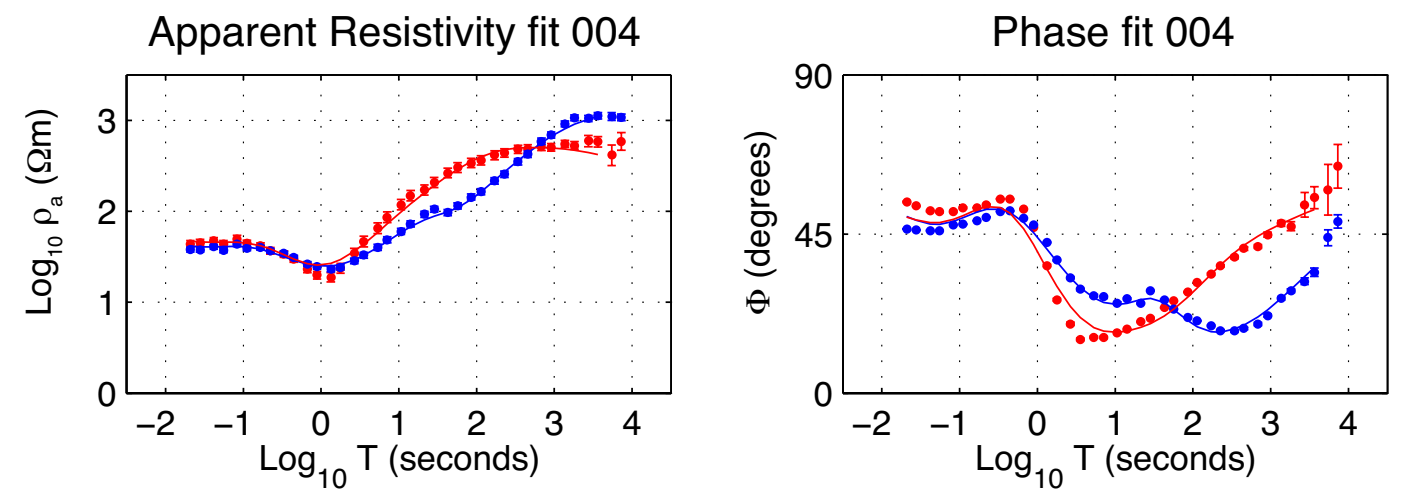

Apparent Resistivity fit 005
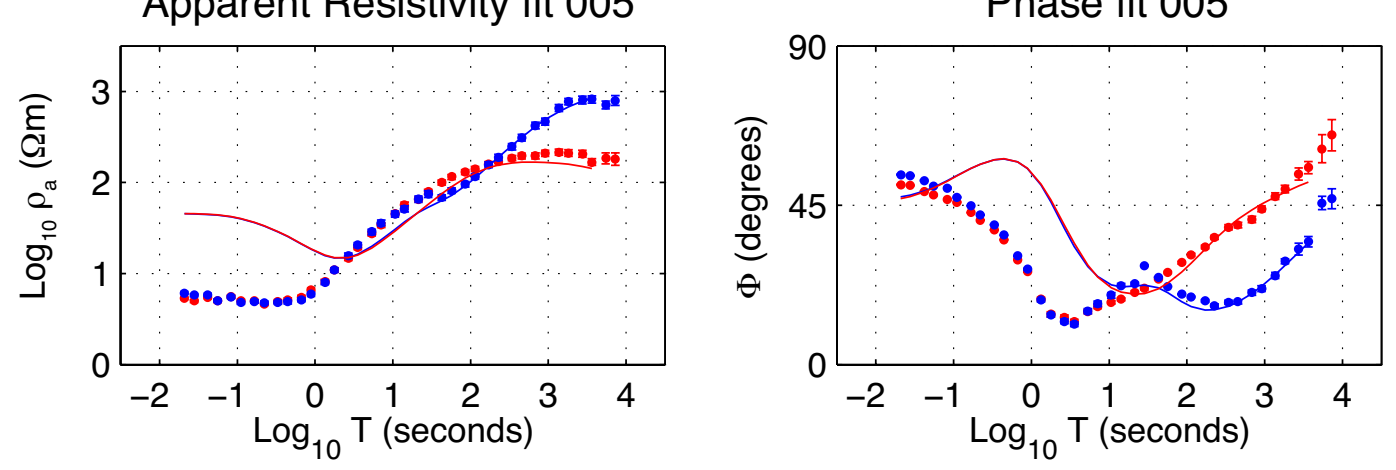

Apparent Resistivity fit 006
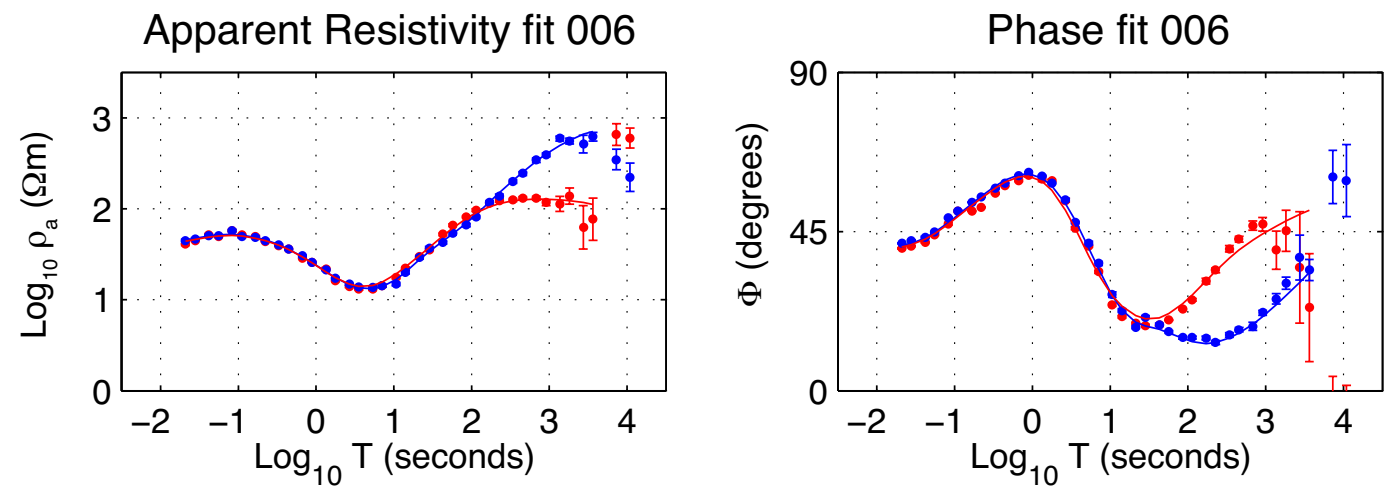

Figure D.12: Fit to inversion model 4 shown in figure D.10 (5\% minimum errors, staticshift and excluding station 005) for stations 004-006. The forward model for the TM mode is shown as a red line and the forward model for the TE mode is shown as a blue line. Because the data for station 005 was excluded from this inversion this station is poorly fitted. 